Bovine lumpy skin disease: epidemiology, economic impact and control opportunities in Ethiopia

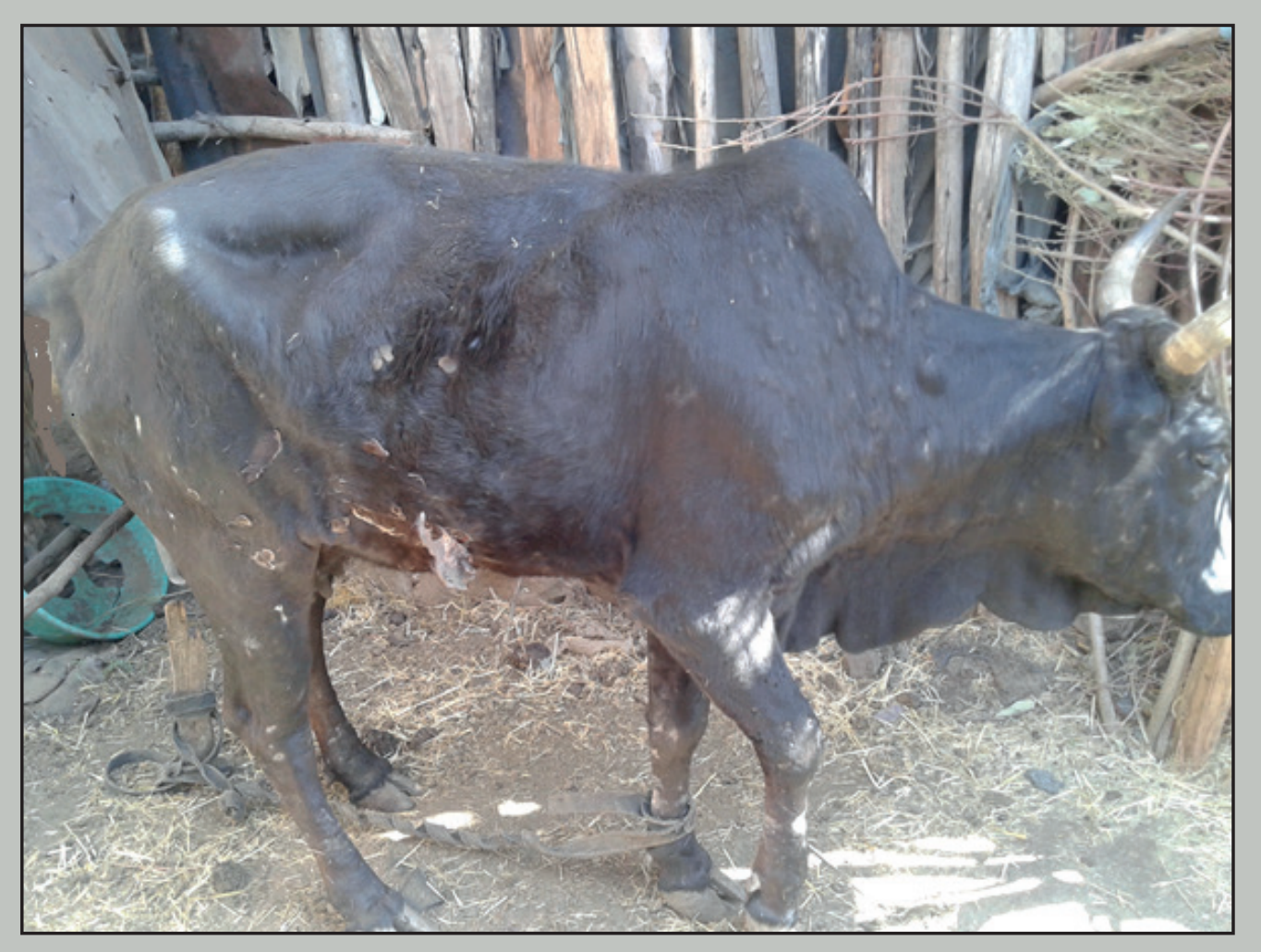

Wassie Molla Abebe 


\section{Propositions}

1. The 5-year cyclic recurrence of lumpy skin disease in the extensive livestock production system is related to waning herd immunity(this thesis).

2. The survival rate of lumpy skin disease virus (LSDV) in the environment greatly influences the infectiousness of an LSDV-infected animal(this thesis).

3. RNA viruses are much more prone to develop resistance to control than DNA viruses.

4. Emergence and re-emergence of many catastrophic diseases are caused by human activities.

5. Massive destruction of eggs containing no or low levels of fipronil is used only to relax consumers' concerns about food safety.

6. Only sick people appreciate the benefit of being healthy.

Propositions belonging to the thesis, entitled

Bovine lumpy skin disease: epidemiology, economic impact and control opportunities in Ethiopia.

Wassie Molla Abebe

Wageningen, 21 February 2018 
Bovine lumpy skin disease: epidemiology, economic impact and control opportunities in Ethiopia

Wassie Molla Abebe 


\section{Thesis committee}

\section{Promotor}

Prof. Dr M.C.M. de Jong

Professor of Quantitative Veterinary Epidemiology

Wageningen University \& Research

\section{Co-promotor}

Dr K. Frankena

Associate professor, Quantitative Veterinary Epidemiology

Wageningen University \& Research

\section{Other members}

Prof. Dr H. Hogeveen, Wageningen University \& Research

Dr S. Gubbins, Pirbright, Woking, UK

Emeritus Prof. Dr A.J. van der Zijpp, Wageningen University \& Research

Prof. Dr J.A. Stegeman, Utrecht University

This research was conducted under the auspices of the Graduate School of Wageningen Institute of Animal Sciences (WIAS). 


\title{
Bovine lumpy skin disease: epidemiology, economic impact and control opportunities in Ethiopia
}

\author{
Wassie Molla Abebe
}

Thesis

submitted in fulfillment of the requirements for the degree of doctor at Wageningen University

by the authority of the Rector Magnificus,

Prof. Dr A.P.J. Mol,

in the presence of the

Thesis Committee appointed by the Academic Board

to be defended in public

on Wednesday 21 February 2018

at 1.30 p.m. in the Aula. 
Wassie Molla Abebe

Bovine lumpy skin disease: epidemiology, economic impact and control opportunities in Ethiopia, 236 pages.

PhD thesis, Wageningen University, Wageningen, the Netherlands (2018)

With references, with summary in English

ISBN 978-94-6343-728-8

DOI https://doi.org/10.18174/430314 


\section{Abstract}

Abebe, W. M. (2018). Bovine lumpy skin disease: epidemiology, economic impact and control opportunities in Ethiopia. PhD thesis, Wageningen University, the Netherlands.

Lumpy skin disease (LSD) is a serious pox disease of cattle caused by LSD virus of the genus Capripoxvirus, subfamily Chordopoxvirinae and family Poxviridae. It is economically an important disease due to its effect on productivity, fertility, animal survival, hide quality, and trade. LSD is an endemic disease in Ethiopia having serious consequences on both national and household incomes. This PhD study has been undertaken with the main aim to generate information on LSD epidemiology, vaccination effect and financial consequences to support the formulation of a disease control strategy. An LSD outbreak occurred at least once in all of the regional states and city administrations in Ethiopia between 2000 and 2015. The average incidence of LSD outbreaks at district level was 5.58 per 16 years ( 0.35 per year). The trend of LSD outbreaks increased over the study period. Outbreaks were frequent at the end of the long rainy season and more numerous in areas with relatively high rain fall. The true animal level and herd level sero-prevalences were estimated as 26.5\% (95\% Cl: 24.7-28.3) and 51.0\% (95\% Cl: 46.8-55.1), respectively. Adult age $(\mathrm{OR}=2.44,(95 \% \mathrm{Cl}: 1.67-3.55))$, contact with other animals (OR=0.41 (95\% Cl: $0.23-$ $0.74)$ ), and presence of water bodies (OR=1.61 (95\% Cl: 1.03-2.52)) were identified as the most important risk factors at individual animal level in relation to LSD seropositivity. The transmission rate between animals in the crop-livestock production system was 0.072 (95\% Cl: 0.068-0.076) per day, whereas the transmission rate among animals in intensive production system was $0.076(95 \% \mathrm{Cl}: 0.068-0.085)$ per day. The reproduction ratio (R) of LSD between animals in the crop-livestock production system was 1.07 and in the intensive production system it was 1.09. Kenyan sheep pox virus strain vaccine (KS1 0-180) significantly reduces the severity of the disease. The vaccine efficacy for susceptibility was estimated to be 0.46 (i.e. a susceptibility effect of 0.54 ) while the infectivity effect of the vaccine was 1.83 . The LSD field outbreak economic impact assessment revealed a total economic loss of USD 1176 per affected herd (USD 489 in the subsistence and USD 2735 in the commercial farm type). The financial analysis showed a positive net profit of USD 136 per herd (USD 56 in the subsistence and USD 283 in the commercial farm type) to LSD vaccine investment. Generally, this PhD research provides insight into the epidemiology, economic impact and control opportunities of LSD in Ethiopia that can support policy makers to formulate control strategy for the disease, which is currently lacking in the country. 



\section{Contents}

Abstract 5

Chapter 1 General introduction 9

Chapter 2 Temporal and spatial distribution of lumpy skin disease

outbreaks in Ethiopia in the period 2000 to 2015

Chapter 3 Seroprevalence and risk factors of lumpy skin disease in

$\begin{array}{ll}\text { Ethiopia } & 57\end{array}$

Chapter 4 Transmission dynamics of lumpy skin disease in Ethiopia $\quad 75$

Chapter 5 Field study on the use of vaccination to control the

occurrence of lumpy skin disease in Ethiopian cattle

Chapter 6 Economic impact of lumpy skin disease and cost

effectiveness of vaccination for the control of outbreaks

in Ethiopia

163

$\begin{array}{lll}\text { Chapter } 7 \text { General discussion } & 195\end{array}$

$\begin{array}{ll}\text { Summary } & 215\end{array}$

About the author $\quad 221$

$\begin{array}{ll}\text { Education and training } & 227\end{array}$

$\begin{array}{ll}\text { Acknowledgements } & 233\end{array}$ 



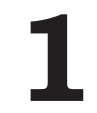

General introduction 



\subsection{Animal disease}

Livestock plays an important role in human societies as a source of food, raw materials, energy, recreation, companionship and income. In the developing world, they also provide organic fertilizer and draft power for crop production and are used as transport and store of wealth (Otte et al., 2004; Bender et al., 2006; Randolph et al., 2007). However, livestock has been threatened by diseases since farming began (Otte et al., 2004; Bender et al., 2006). Animal diseases reduce production and productivity of animals through death, condemnation at slaughter, and by reducing feed conversion, milk yield, weight gain, reproductive capacity and work capacity for ploughing and transport (FAO, 2009). In developing countries, livestock are at risk for a greater range of serious diseases compared to developed countries. In African countries including Ethiopia, transboundary animal diseases such as contagious bovine pleuropneumonia (CBPP), foot and mouth disease (FMD), and lumpy skin disease (LSD) do regularly occur and threaten assets and income of cattle owners (FAO, 2009; AU-IBAR, 2014). Diseases often cause most damage when they occur as an epidemic. Major outbreaks of transboundary animal diseases have been observed in the past, such as FMD in the United kingdom (Thompson et al., 2002), classical swine fever in The Netherlands (Meuwissen et al., 1999) and highly pathogenic avian influenza in Asia and Europe (Otte et al., 2004). These outbreaks have shown the worldwide impact of animal disease in causing significant societal damage by disrupting trade and local and regional economies as well as exacerbating poverty (Otte et al., 2004; FAO, 2009; Oxford-Analytica, 2012).

Little attention, however, has been paid to transboundary animal diseases that prevail in less developed countries, such as LSD. Until recently, LSD has been restricted to Africa and Middle East countries, and currently, the disease has become a threat for European and Asian countries by moving beyond its usual territory. The disease's economic impact is considerable for livestock industry in affected regions and nations (Tuppurainen et al., 2017). Studying and understanding the occurrence, transmission dynamics, consequences for production and the economy, factors associated with the spread, and cost effectiveness of the available intervention measures are essential prerequisites to formulate an appropriate control strategy for such a neglected transboundary animal disease.

\subsection{Lumpy skin disease}

\subsubsection{Characterization and aetiology}

Lumpy skin disease (LSD) is a serious viral cattle disease characterized by fever, nodular lesions, and lymphadenopathy (Weiss, 1968; Woods, 1990; Quinn et 
al., 2002; Radostits et al., 2007; Awad et al., 2010; OIE, 2010; Tuppurainen and Oura, 2012). LSD is caused by lumpy skin disease (Neethling) virus (LSDV), a DNA virus of the family Poxviridea, subfamily Chordopoxvirinae and of the genus Capripoxvirus. The term Neethling has been added to distinguish LSDV from bovine herpes virus 2 which causes pseudo-lumpy skin disease. LSDV has a close antigenic relationship and resemblance to sheep pox and goat pox viruses. These three capripox viruses share a common major antigen on their outer membrane and this similarity is the reason behind the use of sheep pox vaccine against LSD (Woods, 1990; Quinn et al., 2002; Radostits et al., 2007; Babiuk et al., 2008b). So far, LSDV is known to have only one serotype. LSDV is stable between $\mathrm{pH} 6.6$ and 8.6, and it remains viable in the environment and dried scabs for several weeks at ambient temperature. The virus survives at refrigeration temperatures for 6 months, in skin nodules kept at $-80^{\circ} \mathrm{C}$ for 10 years, in air-dried hides for at least 18 days, in necrotic skin nodules over 33 days, and for up to 35 days in desiccated crust (Weiss, 1968; Woods, 1990; OIE, 2013). LSDV is susceptible to direct sunlight but can persist for many months under dark environmental conditions. It is also sensitive to temperature of $55^{\circ} \mathrm{C} / 2$ hours or $65^{\circ} \mathrm{C} / 30$ minutes, highly alkaline or acidic $\mathrm{pH}$, ether (20\%), chloroform, formalin (1\%), detergents containing lipid solvents, iodine compounds (1:33 dilution), phenol (2\%), Virkon ${ }^{\circledR}(2 \%)$, sodium hypochlorite (2-3\%), and quaternary ammonium compounds (0.5\%) (OIE, 2013).

LSDV mainly affects cattle but LSD is also observed in domestic Asian water buffaloes (Ali et al., 1990; FAO, 2015). In wildlife, clinical disease has been suspected in springbok (Antidorcas marsupialis) in Namibia, oryx (Oryx gazelle) in South Africa and Arabian oryx (Oryx leucoryx) in Saudi Arabia (Greth et al., 1992; OIE, 2013). LSDV has been isolated on two separate occasions from springbok antelope in South Africa (Le Goff et al., 2009). Experimentally, LSDV has been shown to infect impala (Aepyceros melampus), giraffe (Giraffe camelopardalis), Grant's gazelle (Gazella thomsonii), goats and sheep (Young et al., 1970; Davies, 1991). Antibodies against LSDV have been found in greater kudu (Tragelaphus strepsiceros), waterbuck (Kobus ellipsiprymnus), impala, reedbuck (Redunca arundinum), giraffe, springbok, eland (Taurotragus oryx), black wildebeest (Connochaetes gnou), blue wildebeest (Connochaetes taurinus), and African buffalo (Syncerus caffer), though their epidemiological role in the maintenance and transmission of the virus is not known (Hedger and Hamblin, 1983; Barnard, 1997; Fagbo et al., 2014).

The clinical signs of LSD have been described under both field and experimental conditions. The incubation period after experimental inoculation is 714 days while it is 2-4 weeks in field outbreaks (Carn and Kitching, 1995a; Radostits et al., 2007). LSD outbreaks present a wide array of signs ranging from inapparent to 
severe disease. Clinical signs that can be exhibited by infected cattle include but are not limited to fever of $40.0-41.5^{\circ} \mathrm{C}$, inflammatory and oedematous swellings of the limbs and abdomen or brisket, cutaneous and mucosal eruption of nodules, enlarged and oedematous regional lymph nodes, drop in milk yield, emaciation, keratitis, and abortion. The nodules are circumscribed areas of 1-5 cm or more in diameter involving the full thickness of the skin and often the subcutis. They can form plaques that can reach more than $10 \mathrm{~cm}$ in diameter. The nodular lesions become necrotic and ulcerate. Some lesions may develop into 'sit-fasts'. Lesions are distributed on all parts of the body from the head to the tail, but most common on the head, neck, udder and perineum. Extensive skin lesions, mouth and respiratory lesions and limb oedema may cause a prolonged anorexia and disinclination to move which in turn may lead to body condition deterioration and finally to death (Woods, 1990; Davies, 1991; Quinn et al., 2002; Kumar, 2011; OIE, 2013).

\subsubsection{Occurrence and transmission}

LSD first appeared in 1929 in Northern Zambia from where it has spread to other parts of Africa and out of the continent (Woods, 1988; Babiuk et al., 2008b). The disease is reported throughout Africa except Libya, Algeria, Morocco and Tunisia which are still considered free of LSD (Tuppurainen and Oura, 2012). LSD has been also reported in most of the Middle East countries following the 1989 outbreak in Israel, which was the first confirmed report outside the African continent (WAHIS; Tuppurainen and Oura, 2012; FAO, 2015; Tuppurainen et al., 2017; Shimshony and Economides, 2006). In recent years, LSD has been reported in central Asian countries (Azerbaijan, Iran and Kazakhstan) and in European countries (Turkey, Cyprus, Greece, Russian Federation, Armenia, Bulgaria, Macedonia, Serbia, Montenegro, and Albania) (WAHIS; FAO, 2015; Tuppurainen et al., 2017). The spread of the disease in Africa took half a century and thus has been slow (Woods, 1990). However, the spread in most of the Middle East countries, Asia and Europe is rapid. The current fast spread of the disease is attributed to uncontrolled animal movement across borders and collapse of veterinary services in the politically unstable Middle East region (FAO, 2015; Tuppurainen et al., 2017).

Morbidity of the disease varies from $5 \%$ to $45 \%$ depending on the immune status of the host and the level of exposure to the pathogen (Woods, 1988; Babiuk et al., 2008b; OIE, 2013; Tuppurainen et al., 2017), but $2-20 \%$ is more common (Davies, 1991). The presence and role of innate resistance towards LSD morbidity has been repeatedly shown in both naturally and experimentally infected cattle (Weiss, 1968; Woods, 1990). LSD mortality is generally low (usually less than $5 \%$ ) but may sometimes reach 20\% (Von Backstrom, 1945; Woods, 1988; Quinn et al., 2002; OIE, 
2010) but also $40-75 \%$ has been reported (Davies, 1991). Recovered animals develop lifelong immunity. The build-up of a susceptible population in the absence of a regular vaccination scheme can lead to recurrence of the disease in an area (Woods, 1990). Usually LSD epidemics recur with an interval of five or six years in unvaccinated cattle populations (Woods, 1988).

LSDV is not considered to be a very contagious virus. Though the virus sheds through many body secretions and excretions of infected animals, direct contact between cattle plays little part in transmission of LSDV. However, cattle can become infected by sharing drinking troughs with severely infected animals and ingestion of feed contaminated with saliva from infected animals (Weiss, 1968; Woods, 1988; Woods, 1990; Radostits et al., 2007; Tuppurainen and Oura, 2012; OIE, 2013). LSDV infected bulls shed LSDV in their semen for prolonged periods (Irons et al., 2005) and the testis and epididymis are sites of persistence of LSDV (Annandale et al., 2010). However, there is no confirmation on the ability of transmission of LSDV through semen and inducing clinical disease after natural mating or artificial insemination (Philpott, 1993; Osuagwuh et al., 2007). Recently, Annandale et al. (2014) demonstrated LSDV transmission followed by clinical disease using semen spiked with a very high virus titre, a titre that has never been detected in semen of naturally infected bulls. Since the shedding of LSDV in bodily secretions is low (Babiuk et al., 2008b), it is unlikely that similar infection rates would be achieved in herds where natural mating or artificial insemination practiced (Annandale et al., 2014). This transmission route is therefore regarded as minor. Lately, intra-uterine transmission of LSDV from an infected pregnant cow to its foetus has been reported (Rouby and Aboulsoud, 2016).

The transmission of LSDV from infected to susceptible cattle is assumed to be predominantly via biting and blood-feeding arthropod vectors. Intravenously feeding arthropods are most responsible for the transmission of LSDV (Carn and Kitching, 1995b). The mosquito Aedes aegypti has been shown to transmit LSDV mechanically from infected to susceptible animals, under experimental conditions (Chihota et al., 2001). Ixodid ticks have also shown to be capable of transmitting LSDV (Tuppurainen et al., 2011). Experimental studies have demonstrated mechanical transmission of LSDV by Rhipicephalus appendiculatus and Amblyomma hebraeum to cattle (Tuppurainen et al., 2011; Tuppurainen et al., 2013b; Lubinga et al., 2015), and vertical (transovarian) transmission by Rhipicephalus decoloratus ticks to its larvae, which in turn transmit the virus to their bovine hosts (Tuppurainen et al., 2013a). Even though transmission of LSDV in a natural setting by specific vectors has not yet been proven clearly, epidemiologic observations strongly suggest that several mosquito and fly species could play a major role. LSDV has been isolated from 
Anopheles stephensi, Culex quinquefasciatus, Culicoides nubeculosus, Biomyia fasciata and Stomoxys calcitrans immediately post-feeding on infected cattle, though the ability of these species to transmit the virus to susceptible cattle still needs to be demonstrated (Weiss, 1968; Woods, 1988; Chihota et al., 2003; OIE, 2013).

A number of animal and environmental factors are associated with the occurrence and spread of LSD: abundance of arthropod vectors, susceptibility of the cattle population, animal movement, wild animals including birds, and rain fall pattern (Woods, 1990). Except animals that have recovered from LSD, all breeds, sexes and ages of cattle are susceptible to the virus infection (Von Backstrom, 1945; Weiss, 1968; Radostits et al., 2007). The severity of LSD depends on the strain of capripoxvirus, the route of virus inoculation and the species, breed, production stage, age, and immune status of the host (Carn and Kitching, 1995b; Quinn et al., 2002; Babiuk et al., 2008a; OIE, 2010; Gari et al., 2011; EFSA AHAW Panel, 2015). Bos taurus is more susceptible to LSDV infection than Bos indicus both in number affected and in severity of the disease. Within Bos taurus, the fine-skinned Channel Island breeds, the Jersey and Guernsey, Friesian and Ayrshire cattle develop more severe disease (Davies, 1991; Radostits et al., 2007; OIE, 2013). Zebu breeds which are indigenous to Africa are generally less susceptible to LSDV (Davies, 1991). Commonly, very young calves, cows at the peak of lactation and malnourished animals are at more risk to develop sever clinical disease (Von Backstrom, 1945; Hunter and Wallace, 2001; OIE, 2010; Tuppurainen et al., 2011; Tuppurainen and Oura, 2012).

Cattle movement is one of the most important factors in the dissemination of LSD whether by trek routes or along roads and railways. Nomadism, communal grazing and watering points (odds ratio $(O R)=4.1$ ), and introduction of new animals to a herd $(O R=8.5)$ are risks for the introduction and spread of LSD (Woods, 1990; Gari et al., 2010; Tuppurainen et al., 2017). Although there is concern that wildlife might serve as a reservoir of LSDV, to date no affected wildlife has been found in natural outbreaks (Woods, 1990; Radostits et al., 2007) and it is generally assumed they do not play an important role in the maintenance and spread of the virus. The red-billed oxpecker (Buphagus erythorhynus) and the egret (Bubulcus ibis) birds are suspected to pick up LSDV through their beaks while they are perching and pecking skin lesions of infected animals and subsequently transmit it mechanically (Woods, 1990).

A warm and humid agro-climate and high densities of vector populations are considered risk factors for the occurrence of LSD (Woods, 1988; Yeruham et al., 1995; Gari et al., 2010). Extensive LSD outbreaks are commonly noticed during the 
wet season and often associated with heavy rain fall and the associated high level of insect activity. In dry seasons, outbreaks tend to be more restricted along water courses in low-lying areas (Weiss, 1968; Woods, 1990; Hunter and Wallace, 2001; Radostits et al., 2007). In dry areas, outbreaks subside and disease tends to disappear at the end of the rainy season. Cold and dry weather causes a sharp reduction in the transmission of LSD which might be associated with reduction of the density of arthropod vectors (Woods, 1990; Davies, 1991). Possibly as a consequence, LSD epidemics are often interspersed with quiescent periods in which there are no apparent clinical cases. Resurgence of LSD is often associated with the onset of rains and the build-up of large numbers of arthropod vectors in the environment (Woods, 1988).

\subsubsection{Diagnosis}

Experienced animal health practitioners can diagnose LSD with a high degree of certainty. Generalized skin nodules in cattle in an endemic area are highly suggestive of LSD (FAO; Quinn et al., 2002; Gelaye et al., 2017). Diagnosis of LSD is often based on characteristic clinical signs. However, sometimes, LSD might be difficult to diagnose as only few skin lesions or transient fever can be present and it may easily be confused with skin lesions due to other diseases such as pseudo-lumpy skin disease, bovine dermatophilosis, onchocerciosis, besnoitiosis, demodex infection, insect bite, and urticaria. Thus, laboratory diagnosis is needed for the confirmation that clinical signs are indeed due to LSDV infections, which is most rapidly done by a polymerase chain reaction (PCR) method (FAO; Awad et al., 2010; OIE, 2010; Gelaye et al., 2017). A variety of molecular tests using capripoxvirusspecific primers are considered sensitive and specific for LSDV identification (Le Goff et al., 2009; Lamien et al., 2011; Gelaye et al., 2013; OIE, 2016). Confirmation can also be performed by electron microscopy and virus isolation using cell culture (Babiuk et al., 2008b). Virus isolation on primary or secondary bovine dermis cells or lamb testis cells culture is considered to be the most sensitive (OIE, 2016). Presence of capripoxvirus antigen can be demonstrated in tissue culture using immunoperoxidase or immunofluorescent staining and the virus can be neutralized using specific antisera (OIE, 2010). Serological assay methods including virus neutralization, indirect enzyme linked immunosorbent assay (iELISA), western blotting, agar gel immunodiffusion test and the indirect fluorescent antibody test are also used to diagnose LSD (Quinn et al., 2002; Awad et al., 2010; OIE, 2010). Virus neutralisation test is the most specific serological test, though it lacks sensitivity to detect infected animals with low levels of neutralising antibody. The Western blot test is sensitive and specific to detect capripoxvirus antibodies, but it is expensive 
and difficult to carry out (OIE, 2016). Serum/virus neutralization tests and electron microscopy examination are widely used as gold standard methods for the detection of capripoxvirus infection (Tuppurainen and Oura, 2012).

\subsubsection{Prevention and control}

LSD spreads into uninfected areas principally by means of wind-borne vectors or movement of infected cattle. Therefore, restriction of cattle movement from infected to uninfected areas and also vaccination are important control measures (Davies, 1991; Radostits et al., 2007). In endemic regions vaccination is the best method of control. Five live attenuated vaccines based on a South African (Neethling) strain of LSDV, Gorgon goat pox virus (Iraq) strain, Kenyan sheep and goat pox virus strain (currently confirmed to be LSDV), Romanian sheep pox strain and Yugoslavian RM 65 sheep pox strain have been used for the control of LSD (FAO; Quinn et al., 2002; Bhanuprakash et al., 2012; Tuppurainen et al., 2014; OIE, 2016). All strains of Capripoxvirus from cattle, sheep and goat origin, share a common major antigen that makes it possible to use sheep pox or goat pox vaccine against LSDV infection in cattle (Kitching et al., 1986; Kitching, 2003). Protection following vaccination is claimed to be lifelong, however, as immunity wanes local LSDV replication will occur at the site of inoculation after challenge (Woods, 1990; OIE, 2010). Calves from naturally infected and vaccinated dams receive passive immunity (by colostrum), which persists for six months (Woods, 1990; Sarma, 2004).

Though vaccination is considered a viable means to control LSDV, incomplete protection and adverse reactions following its use have been reported since long time (Weiss, 1968; Carn, 1993; Abutarbush, 2014). There is an increasing number of vaccine failure reports from various countries associated with the use of capripoxvirus vaccines. Adverse clinical reactions related to the use of the Kenyan sheep and goat pox vaccine have been observed in dairy cattle of Israel (Yeruham et al., 1994), and incomplete protection in dairy herds of Oman (Kumar, 2011) and vaccinated cattle in Ethiopia (Ayelet et al., 2013; Gari et al., 2015). In the Middle East, RM65 vaccine is incriminated for its adverse reactions and incomplete protection in vaccinated cattle (Brenner et al., 2009; Abutarbush et al., 2016). Though the Neethling strain vaccine is also known to cause a local reaction at the injection site (Weiss, 1968) and adverse reactions following administration (Abutarbush et al., 2016), it is claimed to provide a better protection from LSDV infection compared to sheep pox virus and goat pox virus based vaccines (Ben-Gera et al., 2015).

In countries where LSD appears for the first time, all infected and in-contact animals should be stamped-out as soon as possible to avoid the establishment of the disease (Davies, 1991; Tuppurainen and Galon, 2016; Tuppurainen et al., 2017). 
However, stamping-out as the sole control measure is less effective and should be complemented by other control measures such as vaccination (Tuppurainen and Galon, 2016; Tuppurainen et al., 2017). Quarantine and movement control measures are considered helpful (FAO, 2016) but not very effective (Coetzer and Tuppurainen, 2014; Al-Salihi, 2014). Efficient insect control may reduce the rate of LSDV transmission but cannot totally stop it (Al-Salihi, 2014; Tuppurainen and Galon, 2016).

\subsubsection{Economic importance}

Lumpy skin disease is of economic importance to farmers in regions where it is endemic and is a major constraint to international trade in cattle and their products. The disease is associated with cessation or temporary reduction in milk production, temporary or permanent sterility in bulls and cows, low weight gain, damage to hides and death due to secondary bacterial infections, costly control and eradication measures and compulsory animal movement restrictions (Woods, 1988; Chihota et al., 2003; Babiuk et al., 2008a; OIE, 2010; Tuppurainen and Oura, 2012; Elhaig et al., 2017). Secondary infection causes debility and loss of weight, and mastitis (Woods, 1990). Healing is slow and affected cattle often remain debilitated for several months. The economic importance of the disease relates to the prolonged convalescence (Murphy et al., 1999). Abortion losses (up to 10\%) (Coetzer and Tuppurainen, 2014) are not uncommon in the early stages of the disease. Anorexia due to mouth lesions, lameness, pneumonia, cellulitis, and infected lesions cause considerable economic losses in affected herds. The most important losses arise from morbidity, not from mortality (Woods, 1990; Davies, 1991). Though the assessment made so far on the economic significance of the disease is limited, published works indicate $40-65 \%$ milk production loss in intensive milk producing herds (Hunter and Wallace, 2001; Kumar, 2011), and a loss of USD 6.43 per head for local Zebu and USD 58 per head for Holstein-Friesian crossbred cattle in infected herds (Gari et al., 2011). Also a total loss of USD 667,786 in feedlots in and around Adama (Alemayehu et al., 2013) and a loss of $£ 486$ (about USD 800) per animal in a farm (Abutarbush, 2014) due to morbidity and mortality of the disease have been reported. The disease is categorized as a notifiable disease by the World Organization for Animal Health (OIE) because of its substantial economic impact (Tuppurainen and Oura, 2012).

\subsection{Problem statement}

Ethiopia is an agrarian country whose economy is dominated by arable agriculture and livestock production. It has a huge livestock population, particularly 
cattle (about 57 million) (CSA, 2015), but the benefits derived from it are far below its potential. Livestock production contributes $35.6 \%$ of the agricultural GDP, $16.5 \%$ of the national GDP (Metaferia et al., 2011), and 12\% of the annual foreign exchange earnings (NBE, 2014). While low performance is attributable to a number of issues, animal diseases are among the top ranks (APHRD, 2010, 2012). Diseases, especially LSD, limit the productivity (meat, milk, draft power, hides and manure) and international trade of livestock and their products. They are not only bottlenecks for the livestock sector developments in Ethiopia but also threaten food security and exacerbate poverty. LSD is among the well-known animal diseases impairing the Ethiopian farming community to get optimal benefit from their livestock (Rashid and Shank, 1994; APHRD, 2010; Gari et al., 2011; APHRD, 2012).

In Ethiopia, LSD was first observed in the year 1981 in the North western parts of the country (Mebratu et al., 1984). However, it has now (2017) spread to almost all regions and agro-ecological zones of the country with sero-prevalence ranging from 23-31\% at animal level and 26-64\% at herd level (Gari et al., 2010; Gari et al., 2012). The disease has a major economic impact on livestock industry of the nation, as it causes a loss of USD 6.43 and USD 58.00 per head for local Zebu and HolsteinFriesian crossbred cattle, respectively (Gari et al., 2011) and a total loss of USD 667,786 in feedlots in and around Adama due to mortality and rejection (Alemayehu et al., 2013). Although the disease is endemic and causing significant economic losses in the livestock sector, so far, little is known about its spatiotemporal distribution, transmission dynamics, predisposing factors, financial loss at herd level and efficiency of the prevention and control strategies of the disease practiced in Ethiopia. Undertaking research on these issues is crucial for developing effective LSD prevention and control strategies, and enhancement of the optimal contribution of the cattle population to the economies and health of the nation.

\subsection{Aim and outline of the thesis}

The main aim of the studies described in this thesis was to generate knowledge on LSD epidemiology, financial consequence and effect of vaccination as a control means that can be used by policy makers to formulate and optimize the LSD control strategy in Ethiopia. The specific aims were:

(1) to determine the spatial and temporal distribution, and forecast the future pattern of LSD outbreaks;

(2) to estimate the sero-prevalence and identify the associated risk factors of LSD;

(3) to understand LSD epidemic dynamics by quantifying transmission parameters; 
(4) to assess the efficacy of Kenyan sheep pox virus strain vaccine (KS1 0-180) against a natural infection of LSD under field conditions by estimating its effect on the transmission and severity of the disease; and

(5) to estimate the direct economic losses of LSD (mortality loss, milk loss, draft loss) and indirect costs (medication and labour cost) per affected herd, and therewith assess the cost effectiveness of vaccination as a means for LSD control.

The findings may make an important contribution towards the prevention and control of the disease and alleviating its economic consequences.

This thesis is organized into seven chapters, including this general introduction (Chapter 1 ) and a general discussion (Chapter 7 ). The specific objectives were the guide for the outline of the thesis addressed in Chapters 2-6. In Chapter 2, LSD outbreaks time series of the period 2000 to 2015 are described and analysed. The geographical distribution and seasonal pattern of the outbreaks, and the relation between outbreaks and rainfall pattern were assessed. Furthermore, prediction of the original time series data pattern and forecasting of the number of LSD outbreaks expected from January 2016 to December 2018 was done. Chapter 3 concerns a cross-sectional study based on multistage cluster sampling in which sero-prevalence is estimated and associated risk factors are identified. Chapter 4 presents dynamics of LSD outbreaks and transmission parameters between animals using data from field observations. Transmission parameters were estimated using a SIR epidemic model based on data from field observations. Chapter 5 describes the efficacy assessment of KS1 0-180 vaccine against natural LSD occurrence under field conditions and the vaccine impact on the transmission and severity of the disease. Chapter 6 is based on a questionnaire survey to assess LSD induced morbidity and mortality at animal and herd level, herd owners' perception of LSD impact, financial losses from morbidity and mortality, control cost per affected individual animal or herd, and cost effectiveness of vaccination. In the general discussion, Chapter 7, results from Chapters 2-6 are amalgamated to discuss the policy implication of the findings. The chapter ends with suggestions for future research outlook and main conclusions of the thesis.

\section{References}

Abutarbush, S.M., 2014. Efficacy of vaccination against lumpy skin disease in Jordanian cattle. Vet. Rec. 175.

Abutarbush, S.M., Hananeh, W.M., Ramadan, W., Al Sheyab, O.M., Alnajjar, A.R., Al Zoubi, I.G., Knowles, N.J., Bachanek-Bankowska, K., Tuppurainen, E.S., 2016. 
Adverse reactions to field vaccination against lumpy skin disease in Jordan. Transbound. Emerg. Dis. 63, e213-219.

Al-Salihi, K.A., 2014. Lumpy Skin disease: Review of literature. MRSVA 3, 6-23.

Alemayehu, G., Zewde, G., Admassu, B., 2013. Risk assessments of lumpy skin diseases in Borena bull market chain and its implication for livelihoods and international trade. Trop. Anim. Hlth. Prod. 45, 1153-1159.

Ali, A.A., Esmat, M., Attia, H., Selim, A., Abdelhamid, Y.M., 1990. Clinical and pathological studies on lumpy skin disease in Egypt. Vet. Rec. 127, 549-550.

Annandale, C.H., Holm, D.E., Ebersohn, K., Venter, E.H., 2014. Seminal transmission of lumpy skin disease virus in heifers. Transbound. Emerg. Dis. 61, 443-448.

Annandale, C.H., Irons, P.C., Bagla, V.P., Osuagwuh, U.I., Venter, E.H., 2010. Sites of persistence of lumpy skin disease virus in the genital tract of experimentally infected bulls. Reprod.Dom. Anim. 45, 250-255.

APHRD, 2010. Ethiopia Animal Health Yearbook (2009/10), Animal and Plant Health Regulatory Directorate (APHRD), Ministry of Agriculture, Addis Ababa, Ethiopia.

APHRD, 2012. Ethiopia Animal Health Yearbook 2011, Animal and Plant Health Regulatory Directorate (APHRD), Addis Ababa, Ethiopia.

AU-IBAR, 2014. Pan African Animal Resources Yearbook. African Union InterAfrican Bureau for Animal Resources (AU-IBAR) Nairobi, Kenya.

Awad, W.S., Ibrahim, A.K., Mahran, K., Fararh, K.M., Abdel Moniem, M.I., 2010. Evaluation of different diagnostic methods for diagnosis of Lumpy skin disease in cows. Trop. Anim. Hlth. Prod. 42, 777-783.

Ayelet, G., Abate, Y., Sisay, T., Nigussie, H., Gelaye, E., Jemberie, S., Asmare, K., 2013. Lumpy skin disease: preliminary vaccine efficacy assessment and overview on outbreak impact in dairy cattle at Debre Zeit, central Ethiopia. Antivir. Res. 98, 261-265.

Babiuk, S., Bowden, T.R., Boyle, D.B., Wallace, D.B., Kitching, R.P., 2008a. Capripoxviruses: an emerging worldwide threat to sheep, goats and cattle. Transbound. Emerg. Dis. 55, 263-272.

Babiuk, S., Bowden, T.R., Parkyn, G., Dalman, B., Manning, L., Neufeld, J., EmburyHyatt, C., Copps, J., Boyle, D.B., 2008b. Quantification of lumpy skin disease virus following experimental infection in cattle. Transbound. Emerg. Dis. 55, 299-307.

Barnard, B.J.H., 1997. Antibodies against some viruses of domestic animals in southern African wild animals. Onderstepoort J. Vet. Res. 64, 95-110.

Ben-Gera, J., Klement, E., Khinich, E., Stram, Y., Shpigel, N.Y., 2015. Comparison of the efficacy of Neethling lumpy skin disease virus and x10RM65 sheep-pox 
live attenuated vaccines for the prevention of lumpy skin disease - The results of a randomized controlled field study. Vaccine 33, 4837-4842.

Bender, J.B., Hueston, W., Osterholm, M., 2006. Recent animal disease outbreaks and their impact on human populations. J. Agromedicine 11, 5-15.

Bhanuprakash, V., Hosamani, M., Venkatesan, G., Balamurugan, V., Yogisharadhya, R., Singh, R.K., 2012. Animal poxvirus vaccines: a comprehensive review. Expert Rev. Vaccines 11, 1355-1374.

Brenner, J., Bellaiche, M., Gross, E., Elad, D., Oved, Z., Haimovitz, M., Wasserman, A., Friedgut, O., Stram, Y., Bumbarov, V., Yadin, H., 2009. Appearance of skin lesions in cattle populations vaccinated against lumpy skin disease: statutory challenge. Vaccine 27, 1500-1503.

Carn, 1993. Control of capripoxvirus infections. Vaccine 11, 1275-1279.

Carn, V.M., Kitching, R.P., 1995a. The clinical response of cattle experimentally infected with lumpy skin disease (Neethling) virus. Arch. Virol. 140, 503-513.

Carn, V.M., Kitching, R.P., 1995b. An investigation of possible routes of transmission of lumpy skin disease virus (Neethling). Epidemiol. Infect. 114, 219-226.

Chihota, C.M., Rennie, L.F., Kitching, R.P., Mellor, P.S., 2001. Mechanical transmission of lumpy skin disease virus by Aedes aegypti (Diptera: Culicidae). Epidemiol. Infect. 126, 317-321.

Chihota, C.M., Rennie, L.F., Kitching, R.P., Mellor, P.S., 2003. Attempted mechanical transmission of lumpy skin disease virus by biting insects. Med. Vet. Entomol. 17, 294-300.

Coetzer, J.A.W., Tuppurainen, E., 2014. Lumpy skin disease. In African Veterinary Information Portal (AfriVIP). Livestock Health, Management and Production.http://www.afrivip.org/sites/default/files/Isd complete 0.pdf. Accessed 26 June 2017.

CSA, 2015. Agricultural Sample Survey, 2014/15 (2007 E.C.), Volume II: Report on Livestock and Livestock Characteristics (Private peasant holdings). Statistical Bulletin 578. Central Statistical Agency (CSA), Federal Democratic Republic of Ethiopia, Addis Ababa.

Davies, F.G., 1991. Lumpy skin disease, an African capripox virus disease of cattle. Br. Vet. J. 147, 489-503.

EFSA AHAW Panel, E.P.o.A.H.a.W., 2015. Scientific Opinion on lumpy skin disease. EFSA Journal, 13.

Elhaig, M.M., Selim, A., Mahmoud, M., 2017. Lumpy skin disease in cattle: Frequency of occurrence in a dairy farm and a preliminary assessment of its possible impact on Egyptian buffaloes. Onderstepoort J. Vet. Res. 84, 1. 
Fagbo, S., Coetzer, J.A.W., Venter, E.H., 2014. Seroprevalence of Rift Valley fever and lumpy skin disease in African buffalo (Syncerus caffer) in the Kruger National Park and Hluhluwe-iMfolozi Park, South Africa. J. S. Afr. Vet. Assoc. 85, 1.

FAO, Lumpy skin disease of cattle: a growing problem in Africa and the near East. http://www.fao.org/docrep/u4900t/u4900t0d.htm. Accessed 26 April 2017.

FAO, 2009. The state of food and agriculture: Livestock in the balance. FAO Rome, Italy.

FAO, 2015. Emergence of lumpy skin disease (LSD) in Europe. 1-4.

FAO, 2016. Emergence of lumpy skin disease in Asia and Europe. Empres360 animal health, 46. http://www.fao.org/3/a-i6184e.pdf. Accessed on 25 July 2017.

Gari, G., Abie, G., Gizaw, D., Wubete, A., Kidane, M., Asgedom, H., Bayissa, B., Ayelet, G., Oura, C.A., Roger, F., Tuppurainen, E.S., 2015. Evaluation of the safety, immunogenicity and efficacy of three capripoxvirus vaccine strains against lumpy skin disease virus. Vaccine 33, 3256-3261.

Gari, G., Bonnet, P., Roger, F., Waret-Szkuta, A., 2011. Epidemiological aspects and financial impact of lumpy skin disease in Ethiopia. Prev. Vet. Med. 102, 274283.

Gari, G., Grosbois, V., Waret-Szkuta, A., Babiuk, S., Jacquiet, P., Roger, F., 2012. Lumpy skin disease in Ethiopia: seroprevalence study across different agroclimate zones. Acta Trop. 123, 101-106.

Gari, G., Waret-Szkuta, A., Grosbois, V., Jacquiet, P., Roger, F., 2010. Risk factors associated with observed clinical lumpy skin disease in Ethiopia. Epidemiol. Infect. 138, 1657-1666.

Gelaye, E., Lamien, C.E., Silber, R., Tuppurainen, E.S., Grabherr, R., Diallo, A., 2013. Development of a cost-effective method for capripoxvirus genotyping using snapback primer and dsDNA intercalating dye. PloS one 8, e75971.

Gelaye, E., Mach, L., Kolodziejek, J., Grabherr, R., Loitsch, A., Achenbach, J.E., Nowotny, N., Diallo, A., Lamien, C.E., 2017. A novel HRM assay for the simultaneous detection and differentiation of eight poxviruses of medical and veterinary importance. Sci. Rep. 7, 42892.

Greth, A., Gourreau, J.M., Vassart, M., Nguyen-Ba-Vy, Wyers, M., Lefevre, P.C., 1992. Capripoxvirus Disease in an Arabian Oryx (Oryx leucoryx) from Saudi Arabia. J. Wildlife Dis. 28, 295-300.

Hedger, R.S., Hamblin, C., 1983. Neutralising antibodies to lumpy skin disease virus in African wildlife. Comp. Immun. Microbiol. Infect. Dis. 6, 209-213.

Hunter, P., Wallace, D., 2001. Lumpy skin disease in southern Africa: a review of the disease and aspects of control. J. S. Afr. Vet. Assoc. 72, 68-71. 
Irons, P.C., Tuppurainen, E.S., Venter, E.H., 2005. Excretion of lumpy skin disease virus in bull semen. Theriogenology 63, 1290-1297.

Kitching, R.P., 2003. Vaccines for lumpy skin disease, sheep pox and goat pox. Dev. Biol. 114, 161-167.

Kitching, R.P., Hammond, J.M., Black, D.N., 1986. Studies on the major common precipitating antigen of Capripoxvirus. J. Gen. Virol. 67, 139-148.

Kumar, S.M., 2011. An outbreak of lumpy skin disease in a Holstein dairy herd in Oman: A clinical report. Asian J. Anim. Vet. Adv. 6, 851-859.

Lamien, C.E., Lelenta, M., Goger, W., Silber, R., Tuppurainen, E., Matijevic, M., Luckins, A.G., Diallo, A., 2011. Real time PCR method for simultaneous detection, quantitation and differentiation of capripoxviruses. J. Virol. Methods 171, 134-140.

Le Goff, C., Lamien, C.E., Fakhfakh, E., Chadeyras, A., Aba-Adulugba, E., Libeau, G., Tuppurainen, E., Wallace, D.B., Adam, T., Silber, R., Gulyaz, V., Madani, H., Caufour, P., Hammami, S., Diallo, A., Albina, E., 2009. Capripoxvirus Gprotein-coupled chemokine receptor: a host-range gene suitable for virus animal origin discrimination. J. Gen. Virol. 90, 1967-1977.

Lubinga, J.C., Tuppurainen, E.S., Mahlare, R., Coetzer, J.A., Stoltsz, W.H., Venter, E.H., 2015. Evidence of transstadial and mechanical transmission of lumpy skin disease virus by Amblyomma hebraeum ticks. Transbound. Emerg. Dis. 62, 174-182.

Mebratu, G.Y., Kassa, B., Fikre, Y., Berhanu, B., 1984. Observation on the outbreak of lumpy skin disease in Ethiopia. Rev. Elev. Méd. Vét. Pays Trop. 37, 395-399.

Metaferia, F., Cherenet, T., Gelan, A., Abnet, F., Tesfay, A., Ali, J.A., Gulilat, W., 2011. A Review to Improve Estimation of Livestock Contribution to the National GDP. Ministry of Finance and Economic Development and Ministry of Agriculture, Addis Ababa, Ethiopia.

Meuwissen, M.P.M., Horst, S.H., Huirne, R.B.M., Dijkhuizen, A.A., 1999. A model to estimate the financial consequences of classical swine fever outbreaks: principles and outcomes. Prev. Vet. Med. 42, 249-270.

Murphy, F.A., Gibbs, E.P.J., Horzinek, M.C., Studdert, M.J., 1999. Veterinary Virology. Academic Press, San Diego. pp. 278-291.

NBE, 2014. National Bank annual report of 2013-2014. National Bank of Ethiopia (NBE), Addis Ababa Ethiopia.

OIE, 2010. Manual of diagnostic tests and vaccines for terrestrial animals, chapter 2.4.14, Lumpy skin disease. OIE, Paris. http://web.oie.int/eng/normes/MMANUAL/A Index.htm. Accessed 26 February 2016. 
OIE, 2013. Lumpy skin disease. OIE Technical Disease Cards. OIE.

OIE, 2016. Manual of diagnostic tests and vaccines for terrestrial animals, chapter 2.4.13, Lumpy skin disease. OIE, Paris.

http://www.oie.int/fileadmin/Home/eng/Health standards/tahm/2.04.13 LSD.pdf. Accessed 26 February 2017.

Osuagwuh, U.I., Bagla, V., Venter, E.H., Annandale, C.H., Irons, P.C., 2007. Absence of lumpy skin disease virus in semen of vaccinated bulls following vaccination and subsequent experimental infection. Vaccine 25, 2238-2243.

Otte, M.J., Nugent, R., McLeod, A., 2004. Transboundary animal diseases: Assessment of socio-economic impacts and institutional response. FAO, Livestock information and policy branch, AGAL. Livestock policy discussion paper No. 9.

Oxford-Analytica, 2012. The Costs of Animal Disease. A report produced for the Federation for International Animal Health. Oxford Analytica Ltd, 1-37.

Philpott, M., 1993. Dangers of disease transmission by artificial insemination and embryo transfer. Br. Vet. J. 149, 339-369.

Quinn, P.J., Markey, B.K., Carter, M.E., Donnelly, W.J., Leonard, F.C., 2002. Veterinary Microbiology and Microbial Disease. Blackwell Science, Great Britain. pp. 335-342.

Radostits, O.M., Gay, C.C., Hinchcliff, K.W., Constable, P.D., 2007. Veterinary Medicine: A Textbook of the Diseases of Cattle, Sheep, Pigs, Goats and Horses. Sounders Elsevier, Spain. pp. 1424-1426.

Randolph, T.F., Schelling, E., Grace, D., Nicholson, C.F., Leroy, J.L., Cole, D.C., Demment, M.W., Omore, A., Zinsstag, J., Ruel, M., 2007. Invited Review: Role of livestock in human nutrition and health for poverty reduction in developing countries. J. Anim. Sci. 85, 2788-2800.

Rashid, M., Shank, R., 1994. Technical Report: Rough Guide to Animal Diseases in Ethiopia. United Nations Development Programme Emergencies Unit for Ethiopia. http://www.africa.upenn.edu/eue web/diseas94.htm. Accessed 23 August, 2017.

Rouby, S., Aboulsoud, E., 2016. Evidence of intrauterine transmission of lumpy skin disease virus. Vet. J. 209, 193-195.

Sarma, D.K., 2004. A Textbook of Veterinary Virology and Viral Diseases. Kalyani Publishers, New Delhi. pp. 179-181.

Shimshony, A., Economides, P., 2006. Diseases prevention and preparedness for animal health emergencies in the Middle East. Rev. Sci. Tech. Off. Int. Epiz. 25, 253-269. 
Thompson, D., Muriel, P., Russell, D., Osborne, P., Bromley, A., Rowland, M., CreighTyte, S., Brown, C., 2002. Economic costs of the foot and mouth disease outbreak in the United Kingdom in 2001. Rev. Sci. Tech. Off. Int. Epiz. 21, 675-687.

Tuppurainen, E., Galon, N., 2016. Lumpy skin disease: current situation in Europe and neighbouring regions and necessary control measures to halt the spread in south-east Europe.http://dx.doi.org/10.20506/TT.2554.

Tuppurainen, E.S., Lubinga, J.C., Stoltsz, W.H., Troskie, M., Carpenter, S.T., Coetzer, J.A., Venter, E.H., Oura, C.A., 2013a. Evidence of vertical transmission of lumpy skin disease virus in Rhipicephalus decoloratus ticks. Ticks and Tickborne Dis. 4, 329-333.

Tuppurainen, E.S., Lubinga, J.C., Stoltsz, W.H., Troskie, M., Carpenter, S.T., Coetzer, J.A., Venter, E.H., Oura, C.A., 2013b. Mechanical transmission of lumpy skin disease virus by Rhipicephalus appendiculatus male ticks. Epidemiol. Infect. $141,425-430$.

Tuppurainen, E.S., Oura, C.A., 2012. Review: lumpy skin disease: an emerging threat to Europe, the Middle East and Asia. Transbound. Emerg. Dis. 59, 40-48.

Tuppurainen, E.S., Pearson, C.R., Bachanek-Bankowska, K., Knowles, N.J., Amareen, S., Frost, L., Henstock, M.R., Lamien, C.E., Diallo, A., Mertens, P.P., 2014. Characterization of sheep pox virus vaccine for cattle against lumpy skin disease virus. Antivir. Res. 109, 1-6.

Tuppurainen, E.S., Stoltsz, W.H., Troskie, M., Wallace, D.B., Oura, C.A., Mellor, P.S., Coetzer, J.A., Venter, E.H., 2011. A potential role for ixodid (hard) tick vectors in the transmission of lumpy skin disease virus in cattle. Transbound. Emerg. Dis. 58, 93-104.

Tuppurainen, E.S., Venter, E.H., Shisler, J.L., Gari, G., Mekonnen, G.A., Juleff, N., Lyons, N.A., De Clercq, K., Upton, C., Bowden, T.R., Babiuk, S., Babiuk, L.A., 2017. Review: capripoxvirus diseases: current status and opportunities for control. Transbound. Emerg. Dis. 64 729-745.

Von Backstrom, U., 1945. Ngamiland cattle disease: Preliminary report on a new disease, the etiological agent being probably of an infectious nature. J. S. Afr. Vet. Assoc. 16, 29-35.

WAHIS, World Animal Health Information System (WAHIS). In: OIE (Ed.).

Weiss, K.E., 1968. Lumpy skin disease virus. Virol. Monogr. 3, 111-131.

Woods, J.A., 1988. Lumpy skin disease- A review. Trop. Anim. Hlth. Prod. 20, 11-17.

Woods, J.A., 1990. Lumpy skin disease virus. In: Dinter, Z., Morein, B. eds. Virus Infections of Ruminants. Elsevier Science publishers B. V., Amesterdam. pp. 53-67. 
Yeruham, I., Nir, O., Braverman, Y., Davidson, M., Grinstein, H., Haymovitch, M., Zamir, O., 1995. Spread of lumpy skin disease in Israel dairy herds. Vet. Rec. 137, 91-93.

Yeruham, I., Perl, S., Nyska, A., Abraham, A., Davidson, M., Haymovitch, M., Zamir, O., Grinstein, H., 1994. Adverse reactions in cattle to a capripox vaccine. Vet. Rec. 135, 330-332.

Young, E., Basson, P.A., Weiss, K.E., 1970. Experimental infection of game animals with lumpy skin disease virus (prototype strain Neethling). Onderstepoort J. Vet. Res. 37, 79-88. 


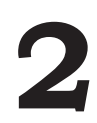

\section{Temporal and spatial distribution of lumpy skin disease outbreaks in Ethiopia in the period 2000 to 2015}

\footnotetext{
W. Molla ${ }^{1,2}$, M.C.M. de Jong ${ }^{1}$, K. Frankena ${ }^{1}$

${ }^{1}$ Quantitative Veterinary Epidemiology, Wageningen University \& Research, Droevendaalsesteeg 1, 6708 PB Wageningen, The Netherlands;

${ }^{2}$ Faculty of Veterinary Medicine, University of Gondar, P.O. Box 196, Gondar, Ethiopia.
}

BMC Veterinary Research (2017) 13: 310

DOI: 10.1186/s12917-017-1247-5 


\begin{abstract}
Lumpy skin disease (LSD) is an infectious viral disease of cattle caused by a virus of the genus Capripoxvirus. LSD was reported for the first time in Ethiopia in 1981 and subsequently became endemic. This time series study was undertaken with the aims of identifying the spatial and temporal distribution of LSD outbreaks and to forecast the future pattern of LSD outbreaks in Ethiopia. A total of 3811 LSD outbreaks were reported in Ethiopia between 2000 and 2015. In this period, LSD was reported at least once in $82 \%$ of the districts $(n=683), 88 \%$ of the administrative zones $(n=77)$, and all of the regional states or city administrations ( $n=9$ and $n=2)$ in the country. The average incidence of LSD outbreaks at district level was 5.58 per 16 years $\left(0.35\right.$ year $\left.^{-1}\right)$. The incidence differed between areas, being the lowest in hot dry lowlands and highest in warm moist highland. The occurrence of LSD outbreaks was found to be seasonal. LSD outbreaks generally have a peak in October and a low in May. The trend of LSD outbreaks indicates a slight, but statistically significant increase over the study period. The monthly precipitation pattern is the reverse of LSD outbreak pattern and they are negatively but non-significantly correlated at lag 0 ( $r=-0.05, p=0.49$, Spearman rank correlation) but the correlation becomes positive and significant when the series are lagged by 1 to 6 months, being the highest at lag $3(r=0.55, p<0.001)$. The forecast for the period 2016-2018 revealed that the highest number of LSD outbreaks will occur in October for all the three years and the lowest in April for the year 2016 and in May for 2017 and 2018. Generally, LSD occurred in all major parts of the country. Outbreaks were high at the end of the long rainy season. Understanding temporal and spatial patterns of LSD and forecasting future occurrences are useful for indicating periods when particular attention should be paid to prevent and control the disease.
\end{abstract}

Key words: Ethiopia, Forecast, Lumpy skin disease, Spatial, Temporal, Time series. 


\subsection{Introduction}

Lumpy skin disease (LSD) is an infectious viral disease of cattle caused by LSD virus of the genus Capripoxvirus and the disease often occurs as epidemics. It has spread from Zambia, where it was first observed in 1929 to most African countries (except Libya, Morocco, Algeria and Tunisia), Middle Eastern countries, and more recently also to European countries (Davies, 1991; Tuppurainen and Oura, 2012; Tuppurainen et al., 2015; Tasioudi et al., 2016; WAHIS, 2016). LSD can occur in diverse ecological zones from the very dry semi-desert, the wet and dry areas to the high altitude temperate areas (Davies, 1991).

LSD was introduced in Ethiopia, for the first time, through north-west (Gojjam and Gondar) in 1981 with subsequent introductions in the West (Wollega) in 1982 from Sudan and in the central part (Shewa) in 1983 (Mebratu et al., 1984). After the introduction, the disease initially spread eastwards, later to all directions and currently it has affected all regions and agro-climatic zones of the country (Mebratu et al., 1984; Gari et al., 2010; APHRD, 2012b). The spread of LSD was enhanced by uncontrolled cattle movements, communal grazing and watering, and pastoralism (Gari et al., 2010; Tuppurainen and Oura, 2012). The poor animal health situation, inefficient prevention and control efforts in combination with late detection of the disease have further contributed to the spread of LSD in Ethiopia (APHRD, 2012b; Tuppurainen and Oura, 2012).

In general, the temporal pattern of disease occurrence can be described with short-term, cyclical and seasonal, and long-term trends; time series analysis is a frequently used method to assess these temporal patterns (Thrusfield, 2007). The cyclical trends are associated with regular, periodic fluctuations in the level of disease occurrence. A seasonal trend is a special case of a cyclical trend, where the periodic fluctuations in disease incidence are related to particular seasons (Thrusfield, 2007). Seasonal variation in the occurrence of infectious diseases is a common phenomenon in both temperate and tropical climates. Seasonal changes in vector abundance are well-known causes of seasonality of vector-borne infections. A good knowledge on the seasonal variation of disease outbreaks has paramount importance for the understanding of the dynamics of the disease and in designing better control strategies (Grassly and Fraser, 2006).

Field observations and experimental studies indicate that blood feeding arthropods are involved as passive vectors in the transmission of LSD virus (Chihota et al., 2001; Tuppurainen and Oura, 2012). The spread of LSD has been frequently associated with epidemics (Carn, 1993). Epidemics of LSD occurred during the rainy season in which the arthropod vector populations are abundant while LSD incidence 
sharply drops during the dry and cold weather seasons (Woods, 1988; Davies, 1991; Wainwright et al., 2013). Seasonal variation in the incidence of LSD outbreaks is common in Ethiopia in which it occurs most frequently between September and December (Ayelet et al., 2014). Resurgence of the disease has been consistently associated with the high rainfall, emergence of large numbers of vectors and a low level of herd immunity (Woods, 1988; Hunter and Wallace, 2001). Epidemics of LSD were reported to recur at intervals of 5 or 6 years (Woods, 1988). The reoccurrence of the disease in provisionally free area is possible when the infection is introduced into the population and the reproduction ratio $(R)$, the average number of secondary cases caused by a single typical infectious individual, becomes greater than one (Dibble et al., 2016).

Animal disease monitoring data is of fundamental importance to know the disease status of a country. In Ethiopia, the disease monitoring is mainly passive as most of the disease outbreaks reported to the federal veterinary services are based on clinical observations (APHRD, 2012a). Monitoring of livestock diseases in the field is the responsibility of regional animal health services, regional veterinary laboratories and district animal health personnel. Disease investigations are generally conducted in response to reports of health problems from livestock owners. There is a regular follow up of disease outbreaks but the monthly livestock disease reporting rate is less than $47 \%$ which is below the required OIE standards of at least 80\% (APHRD, 2010).

Assessing the spatial and temporal patterns is a prerequisite for guiding successful surveillance and control efforts in a country. Therefore, the objectives of this study were to evaluate the spatial and temporal distribution of LSD outbreaks and to forecast future patterns of outbreaks in Ethiopia based on data reported over the period 2000-2015.

\subsection{Materials and methods}

\subsubsection{Study area}

Ethiopia is located in Eastern Africa bordering with Sudan, Eritrea, Djibouti, Somalia, Kenya, and South Sudan. It is a federation of nine member regional states (Tigray, Afar, Amhara, Oromia, Benshangul-Gumuz, Gambella, Southern Nations Nationalities and Peoples Region (SNNP), Harari, and Somali) and two city administrations (Addis Ababa and Dire Dawa). The regional states and city administrations are further divided into zones and the zones into woredas (districts), and the woredas into kebeles. As a whole there are about 15,000 kebeles (5000 urban dwellers associations in towns and 10,000 peasant associations in rural areas) in the country (Tadesse et al., 2010; Mbogo, 2012; GoE, 2016). The country's territory 
presents a diverse topography, ranging from 116 meters below sea level at the Dallol Depression, in the East, to $4620 \mathrm{~m}$ above sea level on the Ras Dashen in the North and covers an area of approximately 1.1 million $\mathrm{km}^{2}$. Ethiopia is broadly divided into three climatic zones: "Kolla" (the hot lowland zone below 1500 m); "Weyna Dega" (mid highland zone between 1500 and 2400 m); and "Dega" (the cool highlands zone above $2400 \mathrm{~m}$ ). Average daily temperature ranges from $20^{\circ} \mathrm{C}$ to $30^{\circ} \mathrm{C}$. Rainfall ranges from $200 \mathrm{~mm}$ to $2000 \mathrm{~mm}$ per year. Ethiopia receives heavy rainfall in June, July and August and occasional showers in February and March. In general, the highlands of Ethiopia receive more rain than the lowlands (Tadesse et al., 2010; GoE, 2016).

The total cattle population of the country is estimated to be about 57 million heads, mostly local breeds (98.7\%); the remaining are hybrid (1.2\%) and exotic breeds $(0.1 \%)$ (CSA, 2015). The livestock production system practiced in the country is usually extensive. In the highland and mid highland, it is highly integrated with crop production where cattle are primarily kept for traction purpose and to provide milk and meat as by-products. In the lowland, where no or little farming is practiced, pastoralists and agro-pastoralists keep cattle to provide mainly milk (Gari et al., 2012; CSA, 2015).

\subsubsection{Outbreak and weather data}

LSD is a notifiable disease and it is required that all occurrences of this disease be reported. LSD outbreak data were obtained from the Federal Veterinary Services Directorate of Ethiopia for the period 2000-2015. The records contained information on place, time, number of cases, number of deaths and number of animals at risk for each month. The reporting format enables calculation of the temporal and spatial distribution of LSD. An outbreak is defined as one or more bovines showing LSD symptoms in a specified geographical area (usually Kebele). During the 16 years period, no significant changes in operation of the veterinary organization that could have affected the level of reporting from the field were noted.

The LSD outbreak incidence was established at district (woredas, $n=683$ ) level using the 16 years outbreak data. The mean LSD outbreak incidence in a district was calculated by summing all reported LSD outbreaks in a district over the study period and divide it by 16 . The geographical distribution of LSD outbreaks over the 16 years was mapped by administrative zone using GIS software QGIS 2.2 (QGIS developer team, Open Source Geospatial Foundation, 2014). The spread of the epidemic was also shown using spmap (South Platte Mapping and Analysis Program, Stata 14) by superimposing the yearly outbreak data onto Ethiopian Woreda 2008 shape files in Microsoft power point program. 
The monthly mean precipitations for the period 1999-2013 were obtained from the Global weather data for SWAT website. From a meteorological point of view, three seasons can be distinguished in Ethiopia; 'Belg' (February to May), 'Kiremt' (June to September) and 'Bega' (October to January). 'Kiremt' is the main rainy season in which the magnitude of rainfall is highest as compared to the other seasons for many parts of the country (NMA, 2013).

\subsubsection{Data analysis}

Data on the number of LSD outbreaks reported each month during the 16year study period were analysed to detect temporal trends and seasonal effects. A simple inspection of the graph of the original LSD outbreak time series was employed to appreciate the presence of a clear long-term trend or seasonal effect. The existence of a long-term trend in LSD outbreaks was modelled by linear regression (Stata version 14) using the number of LSD outbreaks (or trend component of the outbreak) as dependent variable and month of the outbreaks as explanatory variable. Spectral analysis with SAS 9.4 was performed to detect seasonality and cyclical patterns in the LSD outbreak time series.

Decomposition of LSD outbreak time series was performed using package 'TTR' in R software, to identify and estimate the three components of the temporal additive model: seasonality, long-term trend, and irregularity (Coghlan, 2015).

The time series were also seasonally differenced (i.e. deducting the 12 months earlier observation value from each observation value) first followed by first order trend differencing (i.e. deducting the preceding observation value from each observation value) according to the procedure described by Allard (1998) and Coghlan (2015) to make the series stationary (diff function in R). Next autocorrelation analysis (Autocorrelation function in R) was used to assess the seasonality of the differenced time series. The autocorrelation function (ACF) enables to test the significance of seasonality in a time series by examining the ACF correlogram at lags of 12 month intervals (Courtin et al., 2000). The ACF estimates the correlation between the number of outbreaks reported in a given month and the number of outbreaks reported in each of the previous 1 to 192 months. The autocorrelations and partial autocorrelations values of various lags were used for the selection of terms to be included in the initial autoregressive integrated moving average (ARIMA) model (autocorrelations and partial autocorrelations functions in $\mathrm{R})$.

The Holt-Winters exponential smoothing technique as described by Coghlan (2015) was applied to make short term (36 months) forecasts using package 'forecast' in $\mathrm{R}$ software. The possibility of improving the predictive model was 
evaluated by making a correlogram and carrying out the Ljung-Box test on the insample forecast errors for evidence of non-zero autocorrelations at lags 1 to 20. In this method the estimates of the parameters alpha, beta, and gamma represents the level, the slope of the trend component, and the seasonal component, respectively at the current time point. All the three parameters have values between 0 and 1 . Parameter values that are close to 0 indicate that relatively little weight is placed on the most recent observations while forecasting future values.

Exponential smoothing methods are useful for making forecasts, but it does not take into account the correlations between successive values of the time series. However, a better predictive model can be made by taking correlations in the data into account. ARIMA models include an explicit statistical model for the irregular component of a time series that allows for non-zero autocorrelations in the irregular component. An ARIMA $(1,1,1) \times(1,1,1) 12$ model (EmelK; Coghlan, 2015) seemed a plausible model for the LSD outbreak stationary time series and this model was used to forecast the expected numbers of LSD outbreaks for a 36 month (January 2016 to December 2018) future time using the "forecast.Arima()" function in the "forecast" $R$ package. Finally, it was investigated whether or not successive forecast errors of an $\operatorname{ARIMA}(1,1,1) \times(1,1,1) 12$ models were correlated by making a correlogram and carrying out the Ljung-Box test.

The association between monthly rainfall and monthly LSD outbreaks was tested with Spearman rank test (Stata version 14).

\subsection{Results}

\subsubsection{Geographical distribution and incidence of LSD outbreaks}

During the period 2000-2015, LSD has been reported from all regional states $(n=9)$ and city administrations $(n=2)$ of Ethiopia. About $82 \%$ of the districts $(n=683)$ and $88 \%$ of the administrative zones $(n=77)$ in the country reported at least one LSD outbreak in this time period. In total 3811 LSD outbreaks were reported in Ethiopia during the study period (Appendix 2.1: Table S1). Most of these outbreaks were from Oromia (54.5\%), Amhara (27.9\%), SNNP (10.1\%) and Tigray regional states (3.6\%) (Appendix 2.2: Figure S1).

The average incidence of LSD outbreaks at district level was 5.58 over all 16 years or 0.35 per year. The lowest incidences were observed in the eastern lowland (Afar and Somali), southern lowland (Liben), south-west (Benchi Maji) and North (North western zone of Tigray) areas whereas the highest number of outbreaks were documented in the north-west, central, West and south-western parts of the country (Figure 2.1). 


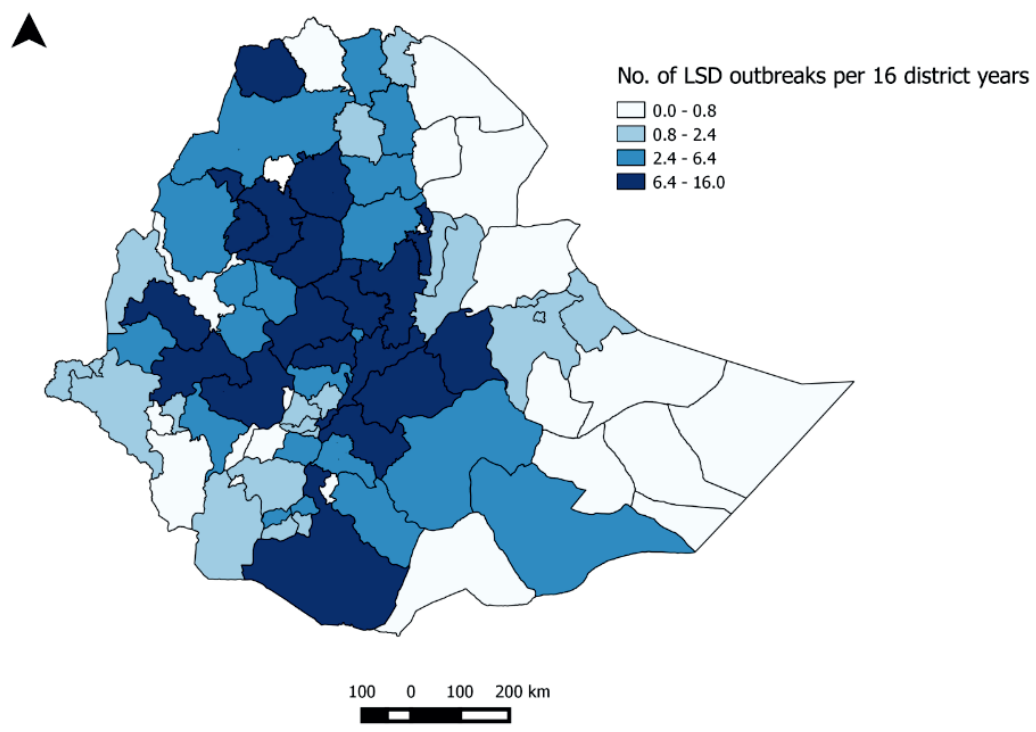

Figure 2.1. Zonal distribution of LSD outbreaks per 16 district years in Ethiopia over the period 2000-2015.

The data shows that LSD affects districts for one or two years and then spreads to other nearby districts/areas with a susceptible cattle population. In this fashion the disease moves from one geographical area to the other and circulates in the country (Appendix 2.2: Figure S2). The reoccurrence of the disease in the study districts varies from 1 year to 13 years, with average length of 4.54 years and median 4 years. The time between outbreaks was shorter in districts geographically located in the West, south-west and central part of the country.

\subsubsection{LSD outbreak time series description and analysis}

The monthly distribution of LSD outbreaks is presented in Figure 2.2, Appendix 2.1: Table S1 and Appendix 2.2: Figure S3. It showed a slight increase in the number of monthly outbreaks which was statistically significant $(P<0.05)$ (Figure 2.2). The seasonality in the numbers of outbreaks is apparent, which tend to be higher in the months following the long rainy season compared to other seasons (Figure 2.2). The undecomposed and undifferenced original LSD outbreak time series was found seasonal by spectral analysis techniques (Figure 2.3). 


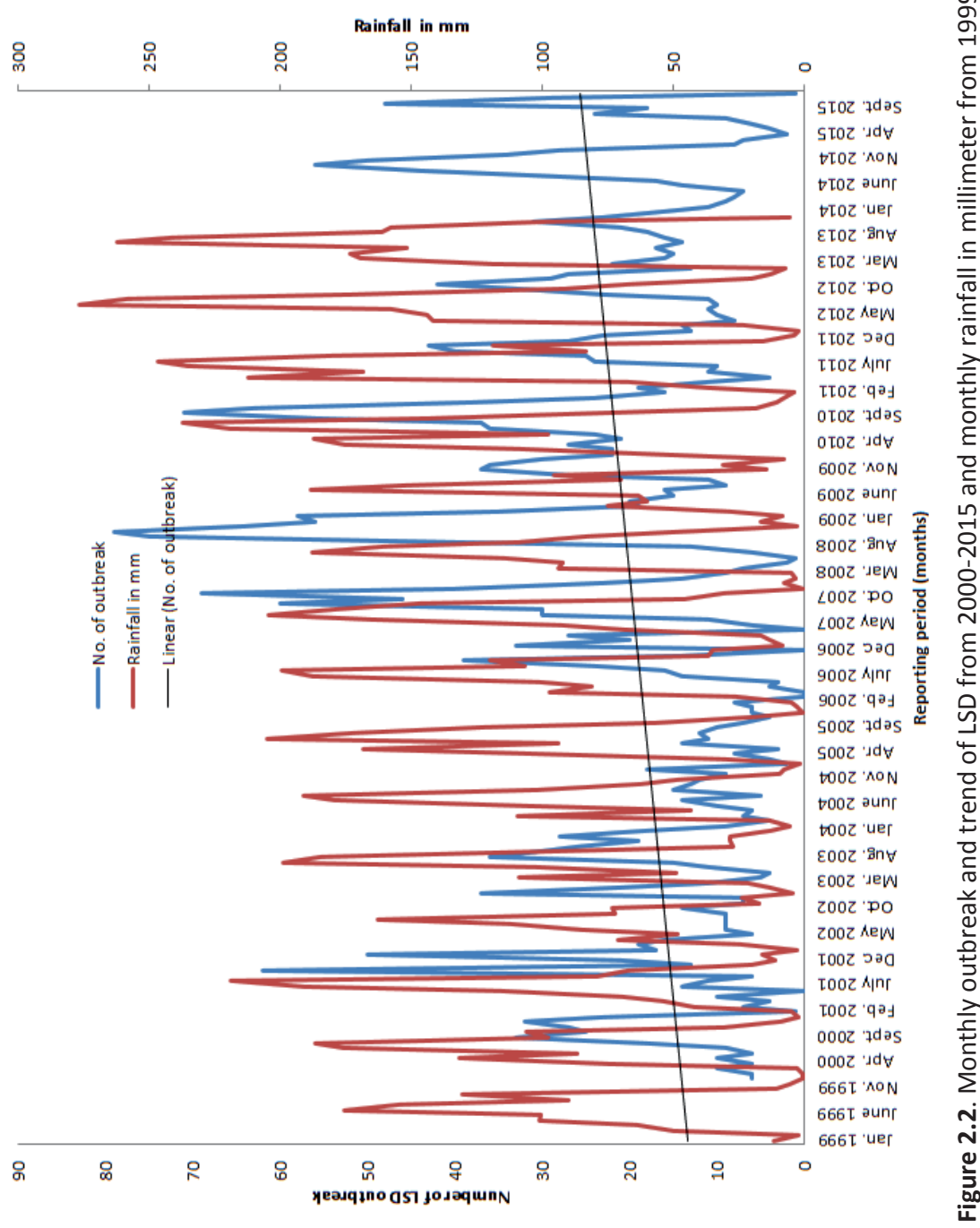




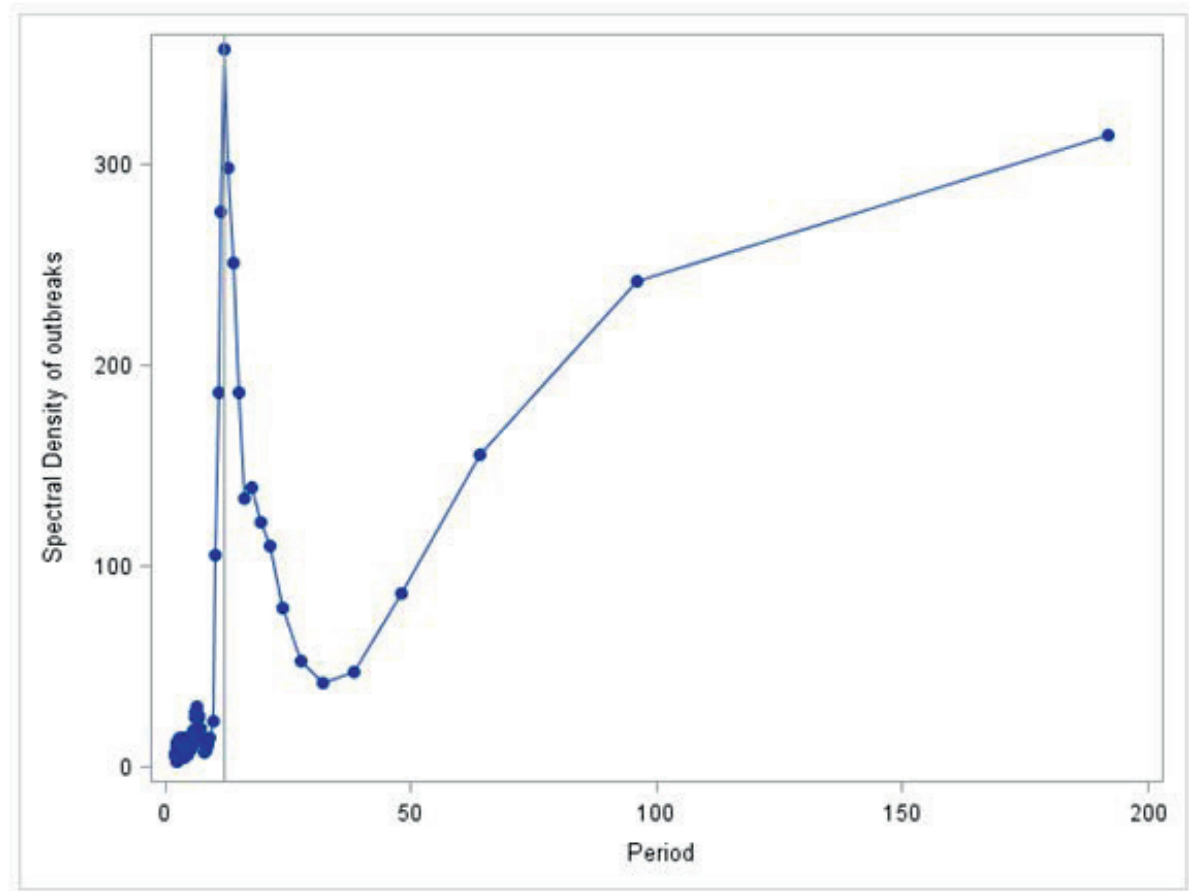

Figure 2.3. Spectral density estimates of LSD outbreaks by month, the vertical reference line at the 12 month period shows the seasonality of the disease.

The trend, seasonal and irregular components of the LSD outbreak time series were estimated by decomposing the time series (Figure 2.4). The estimated trend component shows a decrease from about 20 outbreaks in 2002 to about 6 outbreaks in 2006, followed by a substantial increase to about 41 outbreaks in 2009, decrease to about 16 outbreaks at the end of 2013 and finally increase to about 26 outbreaks in 2014. Though the LSD outbreak pattern from the trend component appears to have a cycle with a periodicity of 5-7 years (peaks in 2002, 2009 and 2014) (Figure 2.4) it was not established by spectral analysis. Linear regression on the trend component of the decomposed time series shows a statistically significant $(p<0.001)$ increase in monthly LSD outbreak numbers between 2000 and 2015.

The seasonal pattern of LSD outbreaks is clearly indicated in Figure 2.3 and 2.4. Seasonal factors were estimated for each month over the 16 year period as the seasonal component of the decomposed LSD time series. The largest seasonal factor is recorded for October (about 16.8) and the lowest for May (about -12.1), indicating that number of LSD outbreaks peaks in October and has a low (trough) in May (Figure 2.4 and 2.5). In general the number of LSD outbreaks was above average for the 
months September to January and below average for February to August (Figure 2.5).

The rainfall season of Ethiopia is indicated in Figure 2.2 and 2.5. The precipitation is above average from April to September and below average from October to March. The rainfall is high in July and August and low in December to February. The precipitation pattern is the reverse of LSD outbreak pattern (Figure $2.5)$, resulting in a negative correlation coefficient ( $r=-0.05, p=0.49$, Spearman rank correlation) at lag 0 and positive correlation coefficients when the series were lagged by 1 to 6 months, the correlation at lag 3 being the highest $(r=0.55, p<0.001)$.

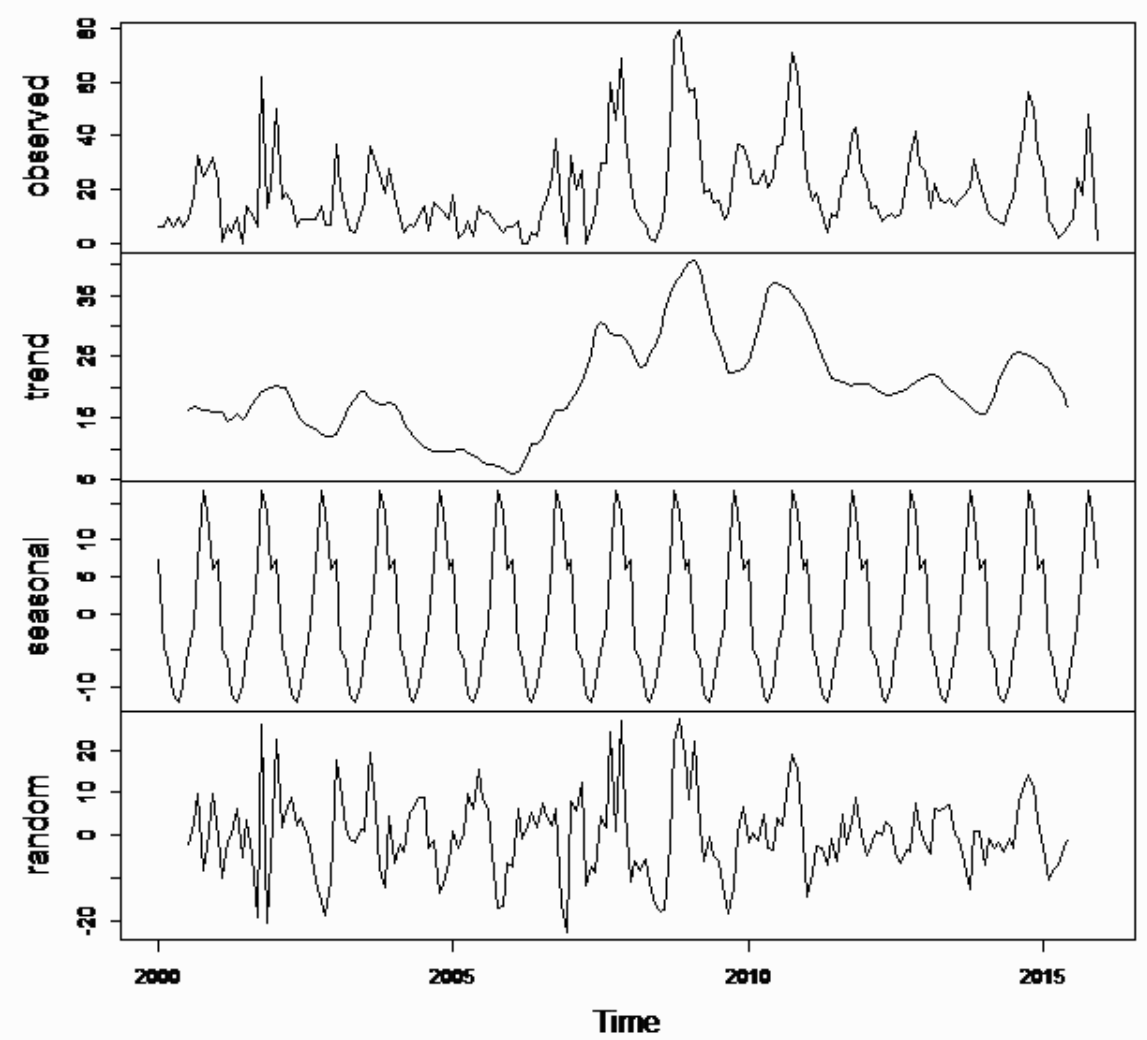

Figure 2.4. Decomposition of the time series of the number of LSD outbreaks (top panel) into three components: trend, seasonality and random. 


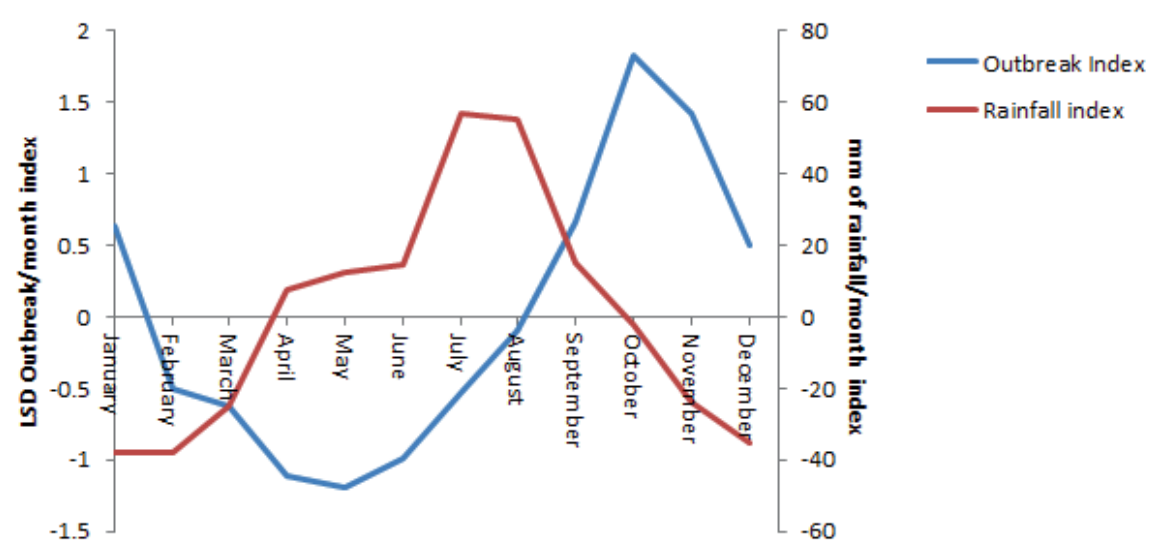

Figure 2.5. Seasonal indices of monthly LSD outbreak between 2000 and 2015 and mean rainfall between 1999 and 2013 in Ethiopia.

\subsubsection{LSD outbreak times series forecasting}

For forecasting with Holt-Winters exponential smoothing, the three parameters: alpha, beta, and gamma which are important for forecasting future values were $0.56,0.00$, and 0.32 , respectively. The original LSD outbreak times series and the forecasted values plotted using Holt's exponential smoothing is shown in Appendix 2.2: Figure S4. The future times, from January 2016 to December 2018 were also forecasted with Holt-Winters' exponential methods (Appendix 2.2: Figure $\mathrm{S} 5)$. However, the correlogram and Ljung-Box test showed the presence of significant $(P=0.002)$ autocorrelations of the in-sample forecast errors at lags $1-20$. This indicates that Holt-Winters exponential smoothing could not provide an adequate forecast.

The LSD outbreak time series was differenced for trend and seasonality, and the resulting series of first order differences appeared to be stationary in mean and variance. The ACF correlogram of first differenced LSD time series indicates significant autocorrelation at lag 1 (-0.349), 12 (-0.468), and 13 (0.273) (Appendix 2.2: Figure S6A). This demonstrates the seasonality of the series because the current monthly value is related to the value of 12 months earlier. The partial correlogram also shows that the partial autocorrelations at lags 1 (-0.349), 9 (-0.199), 12 (-0.487) and $15(0.185)$ exceed the significance bounds (Appendix 2.2: Figure S6B). Hence, the ARIMA model $(1,1,1) \times(1,1,1) 12$ was used for making forecasts for the number of LSD outbreaks from January 2016 to December 2018 (Figure 2.6). The correlogram for the forecasted value shows that none of the sample autocorrelations for lags 1- 
20 exceed the significance bounds, and the p-value for the Ljung-Box test is 0.107 , so it can be concluded that there is little evidence for non-zero autocorrelations in the forecast errors at lags 1-20. Based on the forecast, the highest numbers of LSD outbreaks are expected in October for all predicted years and the lowest in April for 2016 and May for 2017 and 2018 (Appendix 2.1: Table S2).

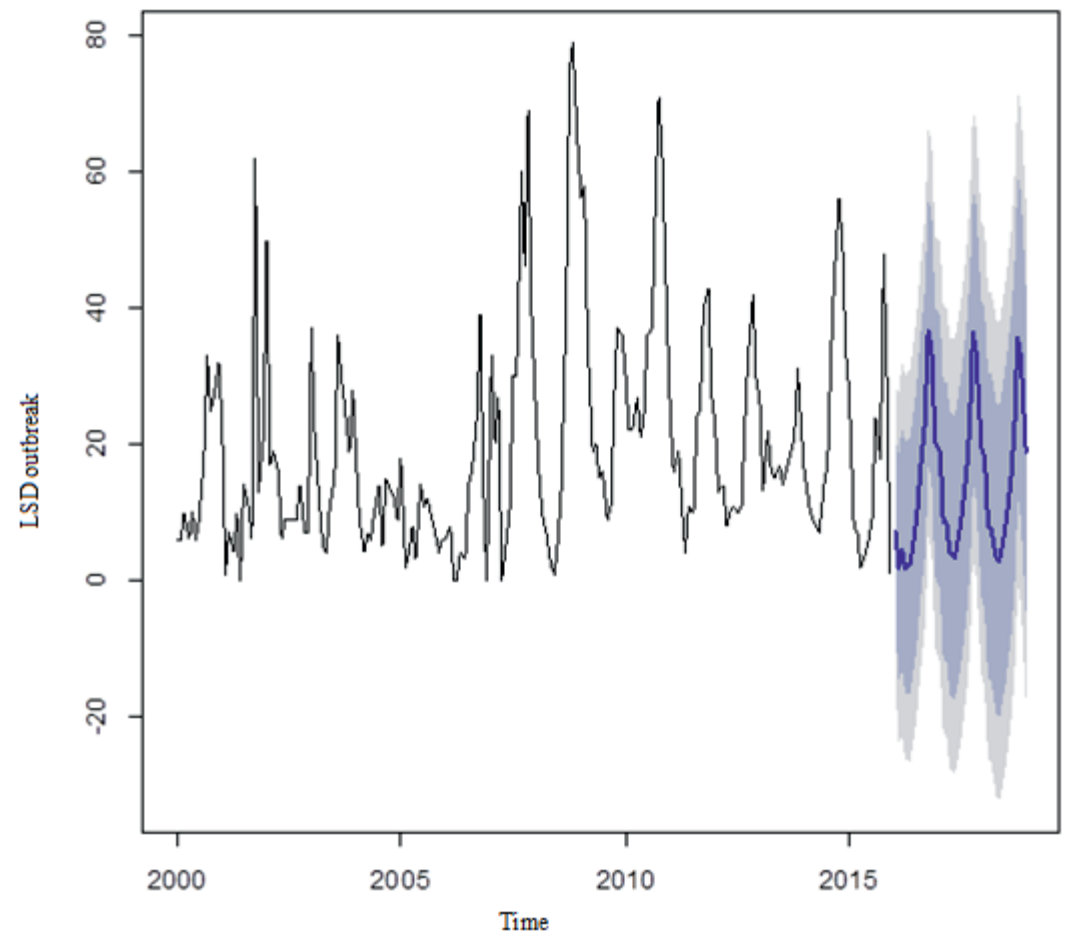

Figure 2.6. The original LSD outbreak time series $(2000-2015)$ and predicted for the next three years (2016-2018) using ARIMA $(1,1,1) \times(1,1,1) 12$ model. The black line represents the original LSD outbreak time series and the blue line the forecasted value. The dark grey shaded area indicate the $80 \%$ confidence interval and the light grey $95 \%$ confidence interval of the predicted values.

\subsection{Discussion}

In the current study, LSD has been recorded from all regional states and city administrations in Ethiopia. A previous retrospective study that covered a period from January 2007 to December 2011 reported no outbreaks from Dire Dawa city administration and Harari regional state (Ayelet et al., 2014). The present study, however, showed that they are affected by the disease. 
Our spatial analysis have shown that distribution of incidence of LSD outbreaks vary among areas (Figure 2.1). The highest LSD incidences were in warm moist highland and the lowest in hot dry lowland areas. This indicate that the parts of the country which receive relatively high rain fall for a reasonable period of time is conducive for the replication and survival of blood feeding arthropods and then for the spread of the disease in the geographical areas (Ecotravelworldwide; Woods, 1990; Gari et al., 2010; Ayelet et al., 2014). The LSD outbreak incidence indicated for the different zones should be treated consciously because under reporting might result in an underestimated incidence.

In this study, it became clear that the occurrence of LSD in an area/districts is sporadic. However, endemicity of the disease is maintained in the country because the outbreaks in different districts/area do not occur at the same time (Appendix 2.2: Figure S2). The average time to reoccurrence was 4.54 years, in line with the 5 yearly reoccurrence of LSD epidemic in unvaccinated populations (Woods, 1990). The reoccurrence was variable across the study districts. Some districts reported outbreaks after one year of quiescence, whereas others reported an outbreak after a much longer period (up to 13 years), which is in line with Gari et al. (2010). This indicates that the disease is not endemic in a district/an area but it occurs in an outbreak (epidemic) form after some years. The reoccurrence is only possible after the seroprevalence (herd immunity) dropped below the critical value and reproduction ratio $(R)$ is above one. How long it will take depends on the rate at which LSD is introduced into the district/area (spark rate) and how far the $\mathrm{R}$ has increased above one (Dibble et al., 2016). The time between outbreaks was shorter in the West and south-west (where rainfall occurs for extended periods of time) and central (where live animal from different parts of the country cross through to the central market) parts of the country.

LSD outbreaks do also not occur at random in time and we demonstrated the seasonality by spectral analysis (Figure 2.3) and estimated a significant autocorrelation between LSD outbreaks at lag 12, 24, 36, etc., indicating the seasonality of the disease. The seasonal pattern of the disease is also clearly indicated in Figure 2.4. The seasonal LSD outbreak variation might be related to the variation in temperature and rainfall between seasons leading to varying arthropod densities in the environment. Seasonal variations in vector abundance, including mosquitoes, ticks, and flies, are well known causes of seasonality for vector borne diseases (Grassly and Fraser, 2006). Identification of temporal patterns can indicate times when particular attention should be paid to control the disease (Thrusfield, 2007). 
The trend of LSD outbreaks from January 2000 to December 2015 indicates a slight, but statistically significant increase over the period (Figure 2.2 and 2.4). This might be attributed to the absence of a specific national strategy for LSD control or eradication (APHRD, 2012a) and the increased tendency of using irrigation for crop cultivation that create favourable environmental conditions for vector borne diseases in the country. The implication of the trend component is that the disease will continue to persist if the environmental circumstances and the poor disease control activities continue.

The positive and significant cross correlations between precipitation and increased LSD outbreaks at lag $3(r=0.55, p<0.001)$ suggests that the rainfall in the previous months are an important factor for the occurrence of LSD outbreaks. The time delay for LSD outbreaks to occur might be justified by the time required for the build-up of arthropod population following the rains (Linthicum et al., 1983; Stewart Ibarra et al., 2013), incubation period (2-4 weeks (Radostits et al., 2007)) of the virus within the cattle host and delay in reporting.

Based on the 2000-2015 reports, the number of LSD outbreaks to occur in each month from 2016 to 2018 was forecasted. The forecast suggests that high number of LSD outbreak will occur from August to January and this is comparable with the available LSD outbreak time series data. The reappearance of the seasonality in the original time series again in the forecasts is an indication of the forecast is reasonable (Allard, 1998). The wide confidence interval (Figure 2.6) indicates the need of frequent updating of the model by incorporating the latest outbreak reports (Allard, 1998). The confidence interval was even wider when HoltWinters' exponential methods were used (Appendix 2.2: Figure S5). The wider confidence interval is related to a limitation of this method, i.e. it does not take the correlations between successive values of the time series into account. The ARIMA model, taking the correlations in the data into account, therefore, is the preferred model to get a reasonable forecast in this study (Coghlan, 2015). The forecasting process can be continued to any point in the future, but will become less reliable for predictions further in time (EmelK). This means we can only gain advantage from the use of short term forecasts. The forecasted results of this study, therefore, will alert and help policy makers to focus on the unusual situations to decide whether any disease control intervention is required to halt the occurrence of the disease in the future.

Currently, Ethiopia has no a well-designed control strategy for LSD (APHRD, 2012a). The animal health authority undertake reactive vaccination campaign using Kenyan sheep pox vaccine when an LSD outbreak is reported somewhere in the country. Vaccination is the only measure taken for LSD control. However, research 
findings indicate that the vaccine used in Ethiopia is not fully protective (Ayelet et al., 2013) which might be the reason for the increase in incidence of LSD outbreaks observed over the current study period. Because there is no regular vaccination program against LSD this might attribute to a drop of herd immunity below the critical point and for the reoccurrence of the disease. We now understood that LSD does not establish endemicity in an area, but it recurs as epidemic in, at average, every 5 years. Therefore, outbreaks might be prevented by bringing the herd immunity above the critical level through vaccination and by prohibiting the entrance of infected animals to the provisionally free area. Vaccination should be undertaken regularly ahead of the onset of the main rainy season with a high coverage. The vaccine currently in use shall be replaced by more competent homologous (Neethling virus) vaccine(Ben-Gera et al., 2015). It is widely agreed that vaccination is the most manageable and realistic approach to control the disease in endemic and resource poor countries. However, to be more effective, the vaccination should be complemented by other additional measures such as movement control.

\subsection{Conclusion}

LSD is wide spread and well established in Ethiopia. It occurred in all regional states and city administrations in the time period between 2000 and 2015. LSD does not establish endemicity in a district, but it does in the country as a whole. It recurs in a district as epidemic, on average in 5 years period. The average incidence of LSD outbreaks at district level was 5.58 over all 16 years. The trend of LSD outbreaks increased over time. Outbreaks are seasonal and occurred more often in the months following the long rainy season. The results of the spatiotemporal analysis and the forecasted value may serve as a guide for the routine surveillance of LSD in the country.

\section{Acknowledgements}

The authors are very much grateful to Nuffic for financing the study. We would like to acknowledge the Ethiopian Veterinary Services Directorate for allowing access to its LSD outbreak report records, and Dr. Bewket Sirawbezu, Dr. Getachew Gari and Dr. Yesmashewa Wogayehu for facilitating access to the database.

\section{References}

Allard, R., 1998. Use of time-series analysis in infectious disease surveillance. Bull. WHO. 76, 327-333. 
APHRD, 2010. Ethiopia Animal Health Yearbook (2009/10), Animal and Plant Health Regulatory Directorate (APHRD), Ministry of Agriculture, Addis Ababa, Ethiopia.

APHRD, 2012a. Biannual epidemiology newsletter, Animal and Plant Health Regulatory Directorate (APHRD), Ministry of Agriculture, Addis Ababa, Ethiopia. 2, 1-21.

APHRD, 2012b. Ethiopia Animal Health Yearbook 2011, Animal and Plant Health Regulatory Directorate (APHRD), Addis Ababa, Ethiopia.

Ayelet, G., Abate, Y., Sisay, T., Nigussie, H., Gelaye, E., Jemberie, S., Asmare, K., 2013. Lumpy skin disease: preliminary vaccine efficacy assessment and overview on outbreak impact in dairy cattle at Debre Zeit, central Ethiopia. Antivir. Res. 98, 261-265.

Ayelet, G., Haftu , R., Jemberie, S., Belay, A., Gelaye, E., Sibhat, B., Skjerve, E., Asmare, K., 2014. Lumpy skin disease in cattle in central Ethiopia: outbreak investigation and isolation and molecular detection of lumpy skin disease virus. Rev. Sci. Tech. Off. Int. Epiz. 33, 877-887.

Ben-Gera, J., Klement, E., Khinich, E., Stram, Y., Shpigel, N.Y., 2015. Comparison of the efficacy of Neethling lumpy skin disease virus and x10RM65 sheep-pox live attenuated vaccines for the prevention of lumpy skin disease - The results of a randomized controlled field study. Vaccine 33, 4837-4842.

Carn, 1993. Control of capripoxvirus infections. Vaccine 11, 1275-1279.

Chihota, C.M., Rennie, L.F., Kitching, R.P., Mellor, P.S., 2001. Mechanical transmission of lumpy skin disease virus by Aedes aegypti (Diptera: Culicidae). Epidemiol. Infect. 126, 317-321.

Coghlan, A., 2015. A Little Book of $R$ For Time Series, Release 0.2. https://media.readthedocs.org/pdf/a-little-book-of-r-fortimeseries/latest/a-little-book-of-r-for-time-series.pdf. Accessed 18 May 2016.

Courtin, F., Carpenter, T.E., Paskin, R.D., Chomel, B.B., 2000. Temporal patterns of domestic and wildlife rabies in central Namibia stock-ranching area, 19861996. Prev. Vet. Med. 43 13-28.

CSA, 2015. Agricultural Sample Survey, 2014/15 (2007 E.C.), Volume II: Report on livestock and livestock characteristics (Private peasant holdings). Statistical Bulletin 578. Central Statistical Agency (CSA), Federal Democratic Republic of Ethiopia, Addis Ababa.

Davies, F.G., 1991. Lumpy skin disease, an African capripox virus disease of cattle. Br. Vet. J 147, 489-503. 
Dibble, C.J., O’Dea, E.B., Park, A.W., Drake, J.M., 2016. Waiting time to infectious disease emergence. J. R. Soc. Interface 13, 20160540.

Ecotravelworldwide, Ethiopia weather and climate zones. http://www.nationalparks-worldwide.info/eaf/ethiopia/ethiopiaweather.html. Accessed 18 May 2016.

EmelK, Time-Series Analysis. https://datajobs.com/data-science-repo/Time-SeriesAnalysis-Guide.pdf. Accessed 10 October 2016.

Gari, G., Grosbois, V., Waret-Szkuta, A., Babiuk, S., Jacquiet, P., Roger, F., 2012. Lumpy skin disease in Ethiopia: seroprevalence study across different agroclimate zones. Acta Trop. 123, 101-106.

Gari, G., Waret-Szkuta, A., Grosbois, V., Jacquiet, P., Roger, F., 2010. Risk factors associated with observed clinical lumpy skin disease in Ethiopia. Epidemiol. Infect. 138, 1657-1666.

GoE, 2016. Ethiopian Government Portal: official web gateway to the government of Ethiopia (GoE). http://www.ethiopia.gov.et. Accessed 8 November, 2016.

Grassly, N.C., Fraser, C., 2006. Seasonal infectious disease epidemiology. Proc. R. Soc. B 273, 2541-2550.

Hunter, P., Wallace, D., 2001. Lumpy skin disease in southern Africa: a review of the disease and aspects of control. J. S. Afr. Vet. Ass. 72, 68-71.

Linthicum, K.J., Davies, F.G., Bailly, C.L., Kairo, A., 1983. Mosquito species succession in a dambo in an East African forest. Mosq. News 43, 464-470.

Mbogo, C.M., 2012. Current status of entomological monitoring and surveillance for an effective delivery of vector control interventions in Ethiopia, produced for review by the United States Agency for International Development. http://pdf.usaid.gov/pdf_docs/PA00J33M.pdf. Accessed 9 July 2016.

Mebratu, G.Y., Kassa, B., Fikre, Y., Berhanu, B., 1984. Observation on the outbreak of lumpy skin disease in Ethiopia. Rev. Elev. Méd. Vét. Pays Trop. 37, 395-399.

NMA, 2013. Annual climate bulletin, National meteorological agency (NMA), Addis ababa

Ethiopia. http://www.ethiomet.gov.et/bulletins/view_pdf/348/2013_annual_bull etin.pdf. Accessed 30 June, 2016.

Radostits, O.M., Gay, C.C., Hinchcliff, K.W., Constable, P.D., 2007. Veterinary Medicine: A Textbook of the Diseases of Cattle, Sheep, Pigs, Goats and Horses. Sounders Elsevier, Spain. pp. 1424-1426.

Stewart Ibarra, A.M., Ryan, S.J., Beltra’n, E., Mejı́a, R., Silva, M., Mun oz, A., 2013 . Dengue Vector Dynamics (Aedes aegypti) Influenced by Climate and Social Factors in Ecuador: Implications for Targeted Control. PloS one 8. 
Tadesse, D., Desta, A., Geyid, A., Girma, W., Fisseha, S., Schmoll, O., 2010. Rapid assessment of drinking water quality in the Federal Democratic Republic of Ethiopia: Country report of the pilot project implementation in 2004-2005. World Health Organization (WHO) and UNICEF. http://www.wssinfo.org/fileadmin/user_upload/resources/RADWQ_Ethio pia.pdf. Accessed 9 July 2016.

Tasioudi, K.E., Antoniou, S.E., Iliadou, P., Sachpatzidis, A., Plevraki, E., Agianniotaki, E.I., Fouki, C., Mangana-Vougiouka, O., Chondrokouki, E., Dile, C., 2016. Emergence of lumpy skin disease in Greece, 2015. Transbound. Emerg. Dis. 63, 260-265.

Thrusfield, M., 2007. Veterinary Epidemiology. Blackwell Science Oxford, UK. pp. 137-151.

Tuppurainen, E.S., Oura, C.A., 2012. Review: lumpy skin disease: an emerging threat to Europe, the Middle East and Asia. Transbound. Emerg. dis. 59, 40-48.

Tuppurainen, E.S., Venter, E.H., Shisler, J.L., Gari, G., Mekonnen, G.A., Juleff, N., Lyons, N.A., De Clercq, K., Upton, C., Bowden, T.R., Babiuk, S., Babiuk, L.A., 2015. Review: Capripoxvirus Diseases: Current Status and Opportunities for Control. Transbound. Emerg. Dis. doi:10.1111/tbed.12444.

WAHIS, 2016. Summary of Immediate notifications and Follow-ups. World Animal Health Information Database (WAHIS interface). http://www.oie.int/wahis_2/public/wahid.php/Diseaseinformation/Immsu mmary. Accessed 18 July 2016.

Wainwright, S., El Idrissi, A., Mattioli, R., Tibbo, M., Njeumi, F., Raizman, E., 2013. Emergence of Lumpy Skin Disease in the Eastern Mediterranean Basin Countries. FAO. http://www.fao.org/docrep/019/aq706e/aq706e.pdf. Accessed on 20 August 2016.

Woods, J.A., 1988. Lumpy skin disease- A review. Trop. Anim. Health Prod. 20, 11-17.

Woods, J.A., 1990. Lumpy skin disease virus. In: Dinter, Z., Morein, B. eds. Virus infections of ruminants. Elsevier Science publishers B. V. Amesterdam. pp. 53-67. 


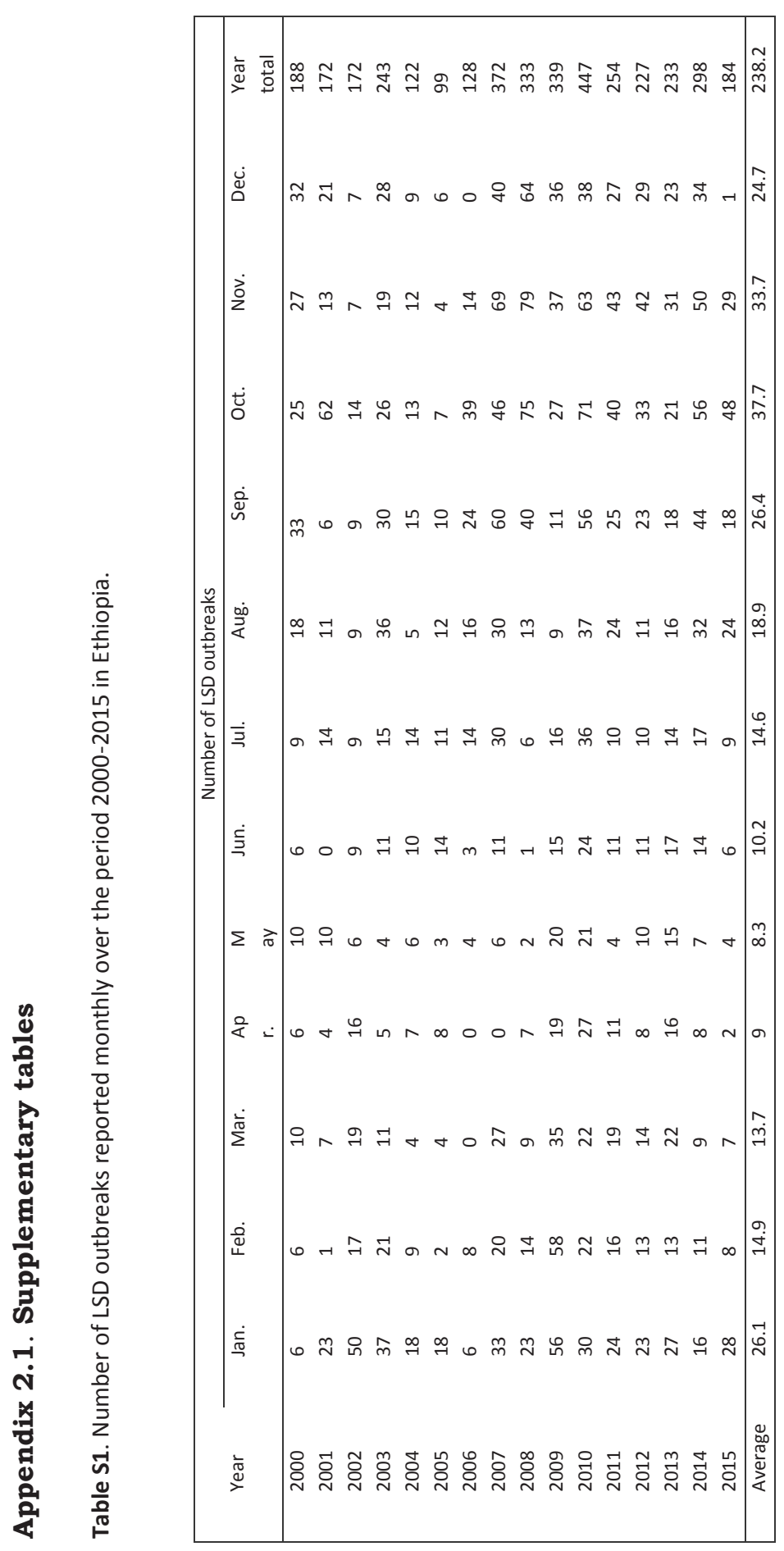


Table S2. 36 month forecast of the number of $\operatorname{LSD}$ outbreaks based on ARIMA $(1,1,1) \times(1,1$, 1)12.

\begin{tabular}{|lccccc|}
\hline Month & $\begin{array}{c}\text { Point } \\
\text { Forecast }\end{array}$ & Lo 80 & Hi 80 & Lo 95 & Hi 95 \\
\hline Jan 2016 & 7.18 & -6.66 & 21.02 & -13.99 & 28.35 \\
Feb 2016 & 1.95 & -14.69 & 18.60 & -23.50 & 27.41 \\
Mar 2016 & 4.63 & -13.20 & 22.47 & -22.64 & 31.91 \\
Apr 2016 & 1.85 & -16.58 & 20.29 & -26.34 & 30.05 \\
May 2016 & 2.32 & -16.46 & 21.11 & -26.40 & 31.05 \\
Jun 2016 & 5.76 & -13.25 & 24.77 & -23.36 & 34.83 \\
Jul 2016 & 9.43 & -9.75 & 28.61 & -19.90 & 38.76 \\
Aug 2016 & 16.01 & -3.30 & 35.33 & -13.53 & 45.55 \\
Sep 2016 & 23.21 & 3.78 & 42.65 & -6.50 & 52.93 \\
Oct 2016 & 36.57 & 17.03 & 56.10 & 6.68 & 66.45 \\
Nov 2016 & 34.15 & 14.51 & 53.78 & 4.11 & 64.18 \\
Dec 2016 & 20.39 & 0.66 & 40.13 & -9.79 & 50.57 \\
Jan 2017 & 18.87 & -1.25 & 38.99 & -11.90 & 49.64 \\
Feb 2017 & 8.75 & -11.63 & 29.13 & -22.41 & 39.92 \\
Mar 2017 & 8.54 & -12.03 & 29.11 & -22.92 & 40.00 \\
Apr 2017 & 3.98 & -16.75 & 24.71 & -27.73 & 35.68 \\
May 2017 & 3.42 & -17.45 & 24.29 & -28.50 & 35.34 \\
Jun 2017 & 6.17 & -14.83 & 27.17 & -25.94 & 38.28 \\
Jul 2017 & 9.42 & -11.70 & 30.54 & -22.87 & 41.72 \\
Aug 2017 & 15.94 & -5.30 & 37.17 & -16.54 & 48.41 \\
Sep 2017 & 22.71 & 1.36 & 44.05 & -9.94 & 55.35 \\
Oct 2017 & 36.32 & 14.86 & 57.77 & 3.50 & 69.13 \\
Nov 2017 & 33.49 & 11.92 & 55.06 & 0.51 & 66.47 \\
Dec 2017 & 19.40 & -2.28 & 41.08 & -13.75 & 52.55 \\
Jan 2018 & 18.02 & -4.10 & 40.14 & -15.81 & 51.85 \\
Feb 2018 & 7.99 & -14.43 & 30.41 & -26.30 & 42.28 \\
Mar 2018 & 7.83 & -14.81 & 30.48 & -26.80 & 42.47 \\
Apr 2018 & 3.30 & -19.53 & 26.13 & -31.62 & 38.22 \\
May 2018 & 2.76 & -20.23 & 25.76 & -32.41 & 37.93 \\
Jun 2018 & 5.53 & -17.62 & 28.67 & -29.87 & 40.92 \\
Jul 2018 & 8.78 & -14.50 & 32.07 & -26.83 & 44.40 \\
Aug 2018 & 15.30 & -8.12 & 38.72 & -20.52 & 51.12 \\
Sep 2018 & 22.08 & -1.48 & 45.64 & -13.95 & 58.11 \\
Oct 2018 & 35.68 & 12.00 & 59.37 & -0.54 & 71.91 \\
Nov 2018 & 32.86 & 9.05 & 56.68 & -3.56 & 69.29 \\
Dec 2018 & 18.78 & -5.16 & 42.73 & -17.84 & 55.40 \\
\hline
\end{tabular}




\section{Appendix 2.2. Supplementary figures}

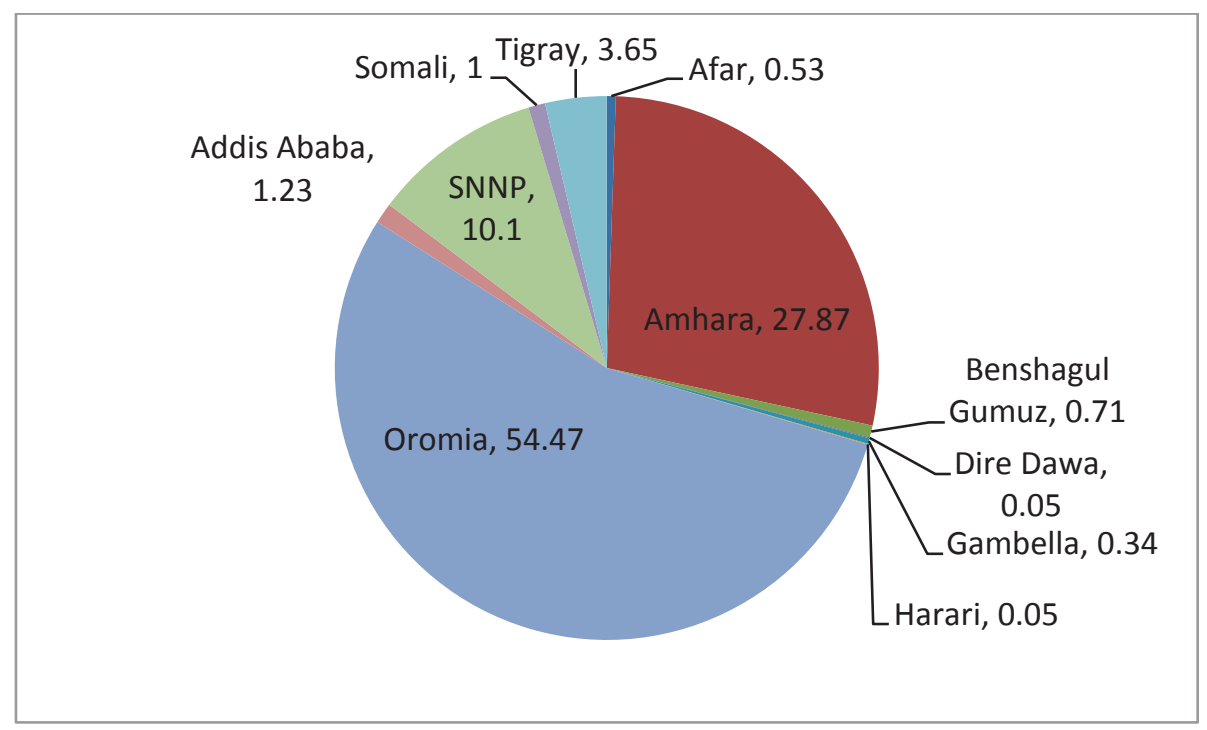

Figure S1. Distribution of LSD outbreaks $(n=3811)$ over regional states and city administrations in the period 2000-2015. 
Figure S2. Animation of the spread of lumpy skin disease epidemics in Ethiopia, 2000-2015

Wassie Molla Abebe

Faculty of Veterinary Medicine / Quantitative Veterinary Epidemiology group

University of Gondar /Wageningen University \& Research

Ethiopia 


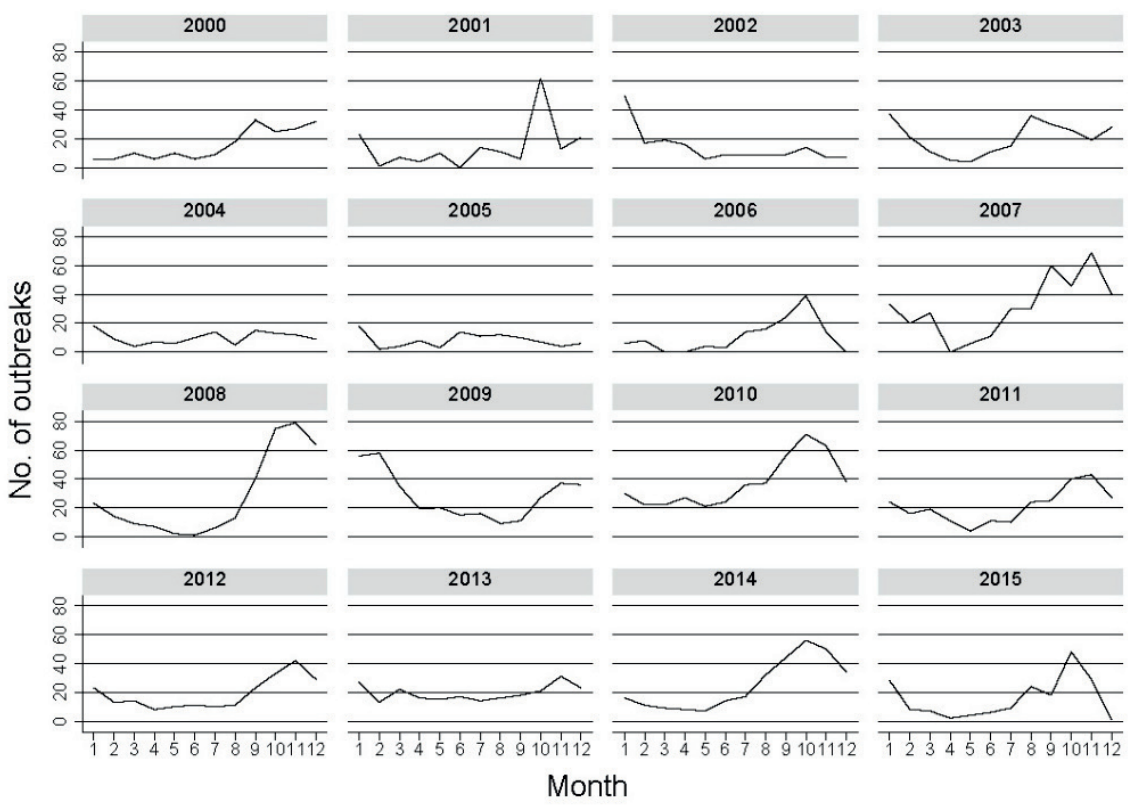

Figure S3. Annual course of LSD outbreaks in Ethiopia, 2000-2015. 


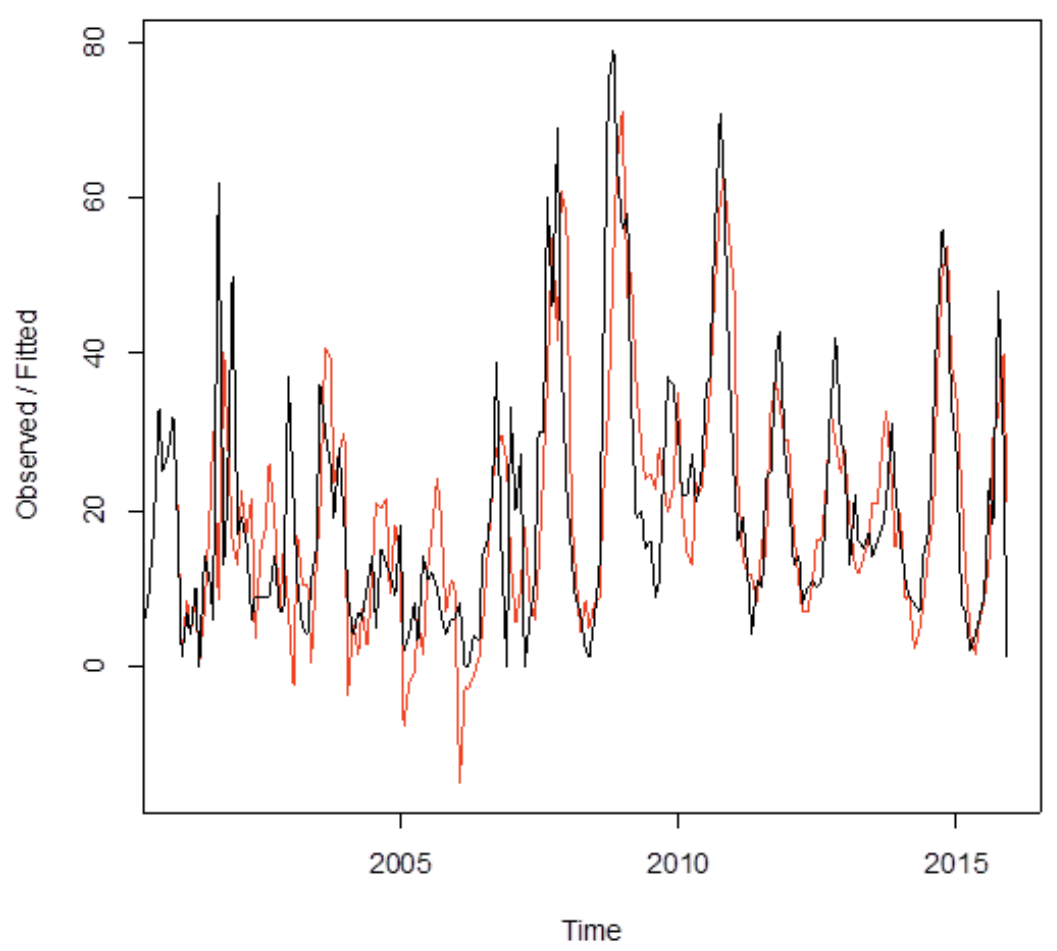

Figure S4. The original LSD outbreak time series (black) and the predicted values (red) using Holt-Winters filtering. 


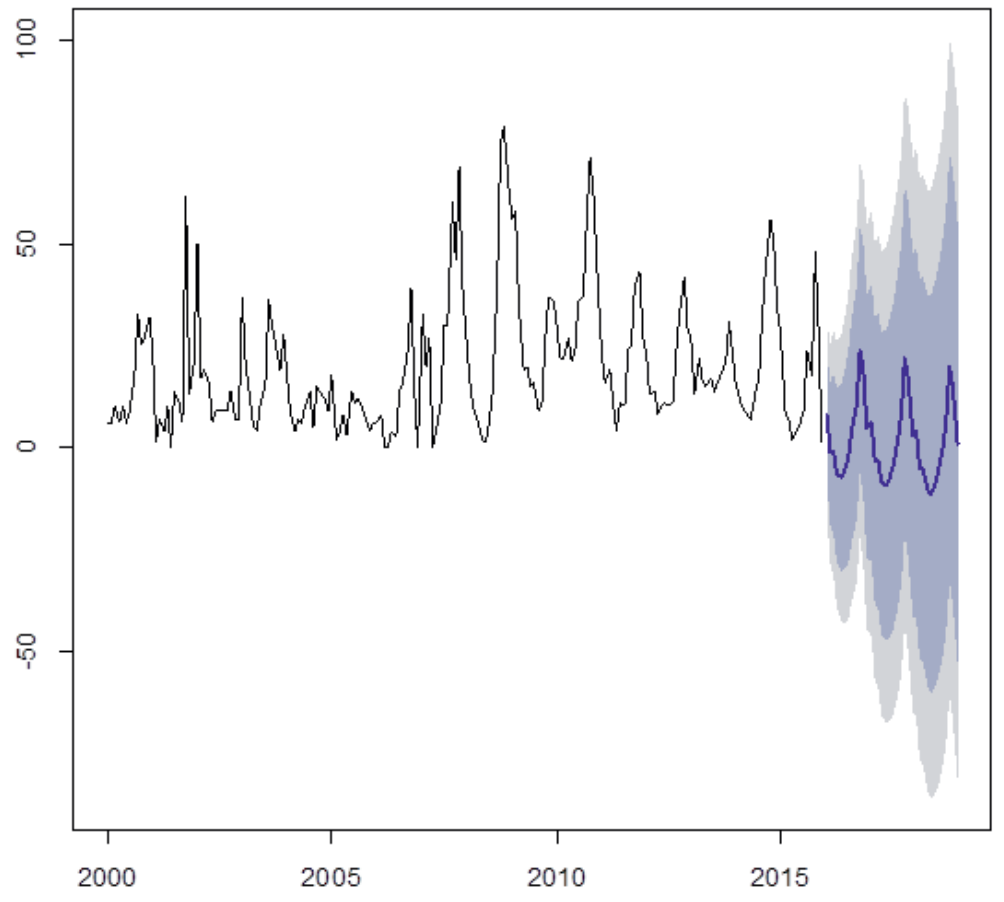

Figure S5. LSD outbreak forecasts based on Holt-Winters analysis for January 2016 to December 2018. The forecasts are shown as a blue line, and the dark grey and light grey shaded areas show $80 \%$ and $95 \%$ prediction intervals, respectively. 

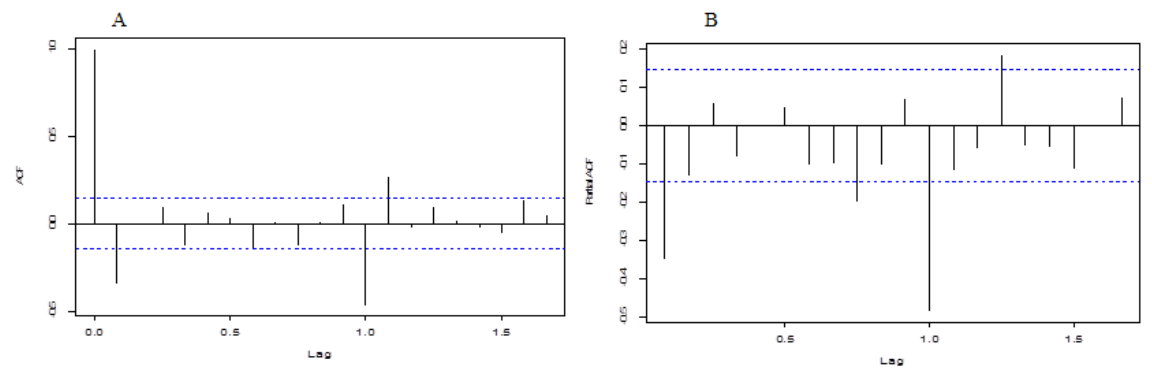

Figure S6. ACF (A) and Partial ACF (B) correlogram after first order seasonal and trend differencing of the original LSD outbreak time series. 


\section{Seroprevalence and risk factors of lumpy skin disease in Ethiopia}

Wassie Molla ${ }^{1,2}$, Klaas Frankena ${ }^{1}$, Getachew Gari ${ }^{3}$, Menbere Kidane ${ }^{3}$, Dereje Shegu ${ }^{3}$, Mart C.M. de Jong ${ }^{1}$

${ }^{1}$ Quantitative Veterinary Epidemiology, Wageningen University \& Research, Droevendaalsesteeg 1, 6708 PB Wageningen, The Netherlands;

2 Faculty of Veterinary Medicine, University of Gondar, P.O. Box 196, Gondar, Ethiopia;

${ }^{3}$ National Animal Health Diagnostic and Investigation Centre (NAHDIC), Sebeta, Ethiopia

Under review 


\begin{abstract}
Lumpy skin disease (LSD) is an acute or inapparent viral disease of cattle endemic in many African and Middle East countries. LSD is one of the major transboundary livestock diseases in Ethiopia. A cross-sectional study using multistage cluster sampling was undertaken in central and north-western parts of Ethiopia with the objectives to estimate seroprevalence and to identify risk factors contributing to the occurrence of the disease. A total of 2386 cattle sera were sampled from 605 herds and 30 clusters (kebeles) located in 10 districts and tested for presence of LSD virus antibodies using virus neutralization test. The overall animal level and herd level apparent seroprevalences were $25.4 \%$ (95\% Cl: 23.7-27.2) and $48.9 \%$ (95\% Cl: 44.9, 52.9), respectively and varied significantly between districts. The true animal level and herd level prevalences were estimated as $26.5 \%$ (95\% Cl: 24.7-28.3) and 51.0\% (95\% Cl: 46.8-55.1), respectively. At individual animal level adult age (OR=2.44 (95\% Cl: 1.67-3.55) compared to calf), contact with other animals (OR=0.41 (95\% Cl: 0.23-0.74), compared to no contact) and presence of water bodies (OR=1.61 (95\% Cl: 1.03-2.52), compared to no such bodies) were identified as the most important risk factors in relation to testing LSD positive. The putative risk factors altitude, breed, sex, and presence of animal trade route showed no significant association with LSD serostatus. The LSD seroprevalence is medium at animal level and high at herd level. Cattle population with many adult animals and live in wet areas are at most risk. The estimated magnitude of seroprevalence and the risk factors identified could be useful inputs for developing a plausible LSD control strategy plan for the country, which is lacking at the moment.
\end{abstract}

Key words: Cattle, Capripoxvirus, Ethiopia, Lumpy skin disease, Risk factors, Seroprevalence 


\subsection{Introduction}

Lumpy skin disease (LSD) is an acute or inapparent viral disease of cattle characterized by fever, nodular lesions on the skin, mucous membranes and internal organs, lymphadenopathy, emaciation, oedema of the limb, and sometimes death (Quinn et al., 2002; Radostits et al., 2007; OIE, 2010; Tuppurainen and Oura, 2012). LSD is caused by Lumpy skin disease (Neethling) virus (LSDV), a DNA virus of the family Poxviridea, subfamily Chordopoxvirinae and of the genus Capripoxvirus. LSDV has close antigenic relationship and resemblance to sheep pox and goat pox viruses (Quinn et al., 2002; Radostits et al., 2007; Babiuk et al., 2008b). So far, LSDV is known to have only one serotype. It is remarkably stable and remains viable in the environment and dried scabs for several weeks at ambient temperature. The virus survives in air-dried hides for at least 18 days, necrotic skin nodules for up to 33 days or longer, and for up to 35 days in desiccated crust (OIE, 2013).

LSD is endemic in many African and Middle East countries and in recent years it has spread to central Asia and South and Eastern Europe. There is a real threat that LSDV will spread further to Western Europe, and further east into Asia (Babiuk et al., 2008b; Tuppurainen and Oura, 2012; FAO, 2015; Tuppurainen et al., 2017).

The transmission of LSDV from infected to susceptible cattle is stated to be predominantly via biting and blood-feeding arthropod vectors. Intravenous feeding are considered the main route for the transmission of LSDV (Carn and Kitching, 1995). Aedes aegypti (a species of mosquito) is capable to transmit LSDV mechanically (Chihota et al., 2001; Chihota et al., 2003; Tuppurainen and Oura, 2012). As a consequence, LSD outbreaks usually occur during the rainy season when insect activity is high (Quinn et al., 2002; Zeynalova et al., 2016). Experimental studies on cattle have also demonstrated mechanical transmission of LSDV by Rhipicephalus appendiculatus and Amblyomma hebraeum, and transovarian transmission by Rhipicephalus decoloratus ticks (Tuppurainen et al., 2011; Tuppurainen et al., 2013a, b; Lubinga et al., 2015). Direct contact between cattle is considered to play a small part in transmission of LSDV. However, cattle can be infected by sharing a drinking trough with severely infected animals (Woods, 1988; Radostits et al., 2007; Tuppurainen and Oura, 2012).

Except animals recovered from LSD, all types and ages of cattle are susceptible to the disease (Radostits et al., 2007). However, very young calves, lactating and malnourished animals are the most at risk. The disease is more severe in cows at the peak of lactation (Hunter and Wallace, 2001; OIE, 2010; Tuppurainen et al., 2011; Tuppurainen and Oura, 2012). The severity of LSD furthermore depends 
on the breed, age, immune status, and the route of virus inoculation (Carn and Kitching, 1995; Quinn et al., 2002; Babiuk et al., 2008a; OIE, 2010; Gari et al., 2011; Tuppurainen et al., 2017). A warm and humid agro-climate, high density of vector populations, communal grazing and watering points, introduction of new animals to a herd and cattle movement are considered to be risk factors of LSD (Woods, 1988; Gari et al., 2010).

LSD has become endemic in Ethiopia since it was reported for the first time in 1981 in north western part of the country (Mebratu et al., 1984). Studies undertaken in various parts of the country indicated prevalence that ranges from $6.4 \%$ to $31 \%$ at animal level and up to $64 \%$ at herd level (Gari et al., 2010; Gari et al., 2012; Abera et al., 2015). The disease has a major economic impact on livestock industry of the nation as it causes reduction in production, death, restriction to the global trade of live animals and animal products, costly control and eradication measures (Radostits et al., 2007; OIE, 2010; Gari et al., 2011; Tuppurainen and Oura, 2012). Despite the high economic losses, so far, little is known about the magnitude of the occurrence and predisposing factors of LSD in the study areas. Hence, this study was undertaken with the aim to estimate the seroprevalence and to identify the risk factors that are contributing to the occurrence of lumpy skin disease.

\subsection{Materials and Methods}

\subsubsection{Study area}

The study was carried out in the central and north-western parts of Ethiopia

(Figure 3.1). In the central part, it was undertaken in Ada'a, Sebeta Hawas, Ambo, Dendi, Debrelibanos, Kuyu and Hidabu Abote districts in Oromia National Regional State. In the north-western part, the sample and the corresponding data were collected from Dejen, Gozamn and Jabitenan districts in Amhara National Regional State. These districts were selected because of their accessibility and their LSD outbreaks in the past. The main livelihood within these districts is crop-livestock mixed farming, which is based on limited communal and/or private grazing areas and the use of crop residue and stubble and which is characterized by smallholdings. Cattle kept in this system primarily provide draft power for crop production, milk for consumption, manure for soil fertility and fuel, and cash income.

\subsubsection{Study design and sampling}

A cross-sectional study with a two-stage sampling technique was carried out from January 2015 to May 2015. Cattle were selected by multistage cluster sampling in which kebele was the primary sampling unit and individual animal the secondary 


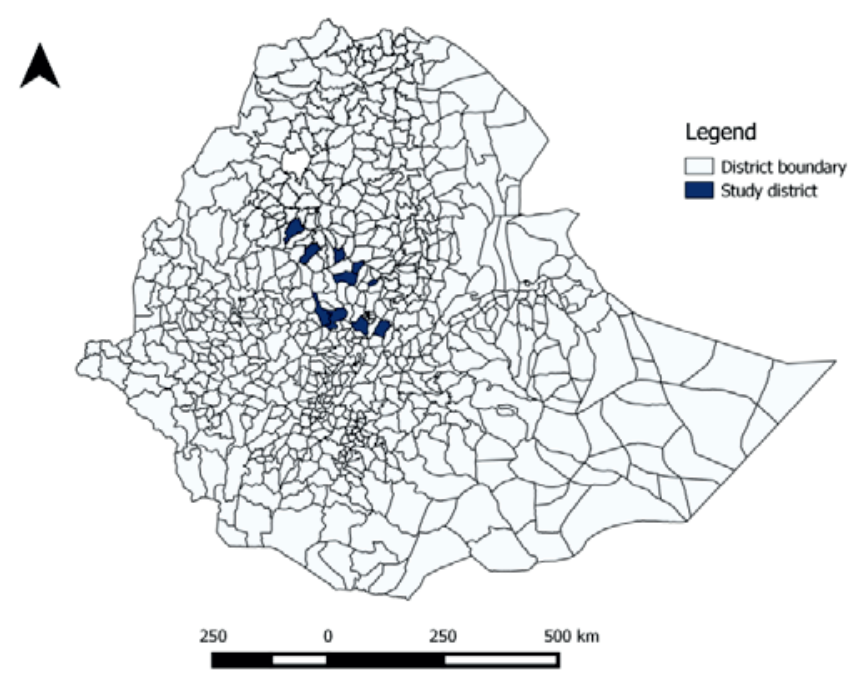

Figure 3.1. Map of Ethiopia showing the study districts.

sampling unit. Multistage cluster sampling was chosen for the practical reason that the population studied has a nested structure and due to lack of individual animal lists in the study area (Thrusfield, 2007). The sample size was calculated using the method described by Bennett et al. ( 1991), Tschopp et al. (2009) and Dohoo et al. (2012).

The sample size to estimate the prevalence with a specified precision is given by:

$$
n=g c=\frac{P(100-P) D}{S E^{2}}
$$

Where $\mathrm{n}$ is the sample size, $\mathrm{p}$ is the prevalence as percentage, $\mathrm{D}$ is the design effect, $\mathrm{SE}$ is the precision (standard error), $\mathrm{g}$ is the average number of individuals sampled per cluster, and $\mathrm{c}$ is the number of clusters sampled. The design effect is given by formula 2 (Bennett et al., 1991):

$$
D=1+(g-1) I C C
$$

The intracluster correlation coefficient (ICC) is a measure of the relatedness of clustered data. Mathematically, it is given by:

$$
\text { ICC }=\frac{\sigma_{u}^{2}}{\sigma_{u}^{2}+\pi^{2} / 3}
$$


Where $\sigma_{u}^{2}$ is the between-cluster component of variance and $\pi^{2} / 3$ the within-cluster component of variance of the logistic distribution.

Gari et al. (2012) reported ICC to be 0.2 for kebele and considering the possibility to collect maximally about 80 (g) serum samples by a team of 4 people per day in a kebele, D equals 17 (formula 2). Sampling 80 animals per cluster (kebele) with an expected disease prevalence of $27 \%$ (Gari et al., 2012) and a desired precision of $3.75 \%$ gave 30 clusters and thus a total sample size of around 2400 . The clusters and the total sample size were more or less equally distributed among 10 study districts. The list of kebeles was obtained from each district agricultural office. Kebeles within district and animals within kebeles were selected randomly. All cattle in a kebele were considered as one population (cluster) since they share common grazing and watering points.

\subsubsection{Potential risk factors}

Data on putative risk factors were obtained by interviewing the farmer (herd owner) at the time of blood sampling. The term "herd" here designates a group of animals owned by a household. The possible explanatory variables included: altitude $(<2000,2000-4000$ and $>4000$ meter above sea level), contact with other animals (yes, no), free animal movement (yes, no), presence of water bodies (river, pond, lake, dam, swampy and irrigated lands) (yes, no), animal trade route in the study area (yes, no), and animal characteristics: breed, age and sex. Individual animals were categorized as calf ( $0.5 \leq 1$ year), young4(1years) and adult ( $\geq 4$ years), breed as Holstein-Friesian cross (HF cross) and local Zebu, sex as male or female.

\subsubsection{Sample collection and laboratory analysis}

Blood samples $(6 \mathrm{ml})$ were collected from the jugular vein of each animal with a history of non-vaccination, using sterile disposable needles and plain vacutainer tubes. The samples were allowed to stand overnight and then the sera were decanted into cryovials. The sera were transported to the National Animal Health Diagnostic and Investigation Centre (NAHDIC) at Sebeta, Ethiopia in ice packs and stored at $-20^{\circ} \mathrm{C}$ until testing for antibodies against LSDV using virus neutralization test (VNT).

The VNT was performed using a constant-virus/varying-serum method (Gari et al., 2008; OIE, 2010). Briefly, each serum was tested in duplicate wells at serial dilutions of $1 / 5,1 / 25,1 / 125,1 / 625$ and $1 / 3125$. Kenyan sheep pox virus (KS1) was used at 100 TCID50 per well in 96-well flat-bottomed tissue-culture microtiter plates. Vero cell was used as culturing host for the test to get consistent results (OIE, 2010). The plates were incubated at $37^{\circ} \mathrm{C}$ in an atmosphere containing $5 \%$ carbon dioxide 
for 9 days. The presence of cytopathic effect (CPE) was examined using an inverted phase-contrast microscope as of day 4 and final reading was made at day 9 . The serum was considered LSD positive, when CPE was inhibited either in both or in one of the duplicate wells at $1 / 25$ or higher dilutions.

\subsubsection{Data management and analysis}

The collected data were entered in to Microsoft Excel spreadsheet, edited and analysed using STATA 14. The animal level and herd level true prevalences were obtained by adjusting the corresponding apparent seroprevalence (AP, as percentage) for $96 \%$ sensitivity (Se) and $100 \%$ specificity (SP) of virus neutralization test (VNT) (Babiuk et al., 2009) using formula 4 (Dohoo et al., 2012; Rogan and Gladen, 1978).

$$
\text { True prevalence }=\frac{\mathrm{AP}+\mathrm{SP}-100}{\mathrm{Se}+\mathrm{SP}-100}
$$

Analysis of the association between animal LSD serostatus and potential risk factors and estimation of variance components were performed by first using univariable mixed effect logistic regression model with kebele as random effect (melogit STATA command). Variables with $P$ values $<0.25$ were checked for multicollinearity between each other using tolerance (collinear at values $<0.4$ ) and variance inflation factor (VIF, collinear at VIF >10 (Dohoo et al., 2012)) tests. If variables were collinear one of them was removed. Next, all potential risk factors were fitted in a multivariable mixed effect logistic regression model and the final model was obtained by a backward stepwise elimination procedure while checking for confounding which was considered present if any of the remaining coefficients changed at least $25 \%$ after removing a non-significant $(p>0.05)$ variable from the model. Interactions were tested for all combinations of the significant main effects. Finally, ICC, SE and Design effect were calculated using formula 1, 2 and 3 to understand the extent to which the observations between clusters are correlated and to assess the precision of the prevalence estimate.

\subsection{Results}

\subsubsection{Seroprevalence}

Descriptive information about the target and study population is presented in Table 3.1. LSDV seropositive animals were detected in all kebeles. Of all animals sampled, 607 (25.4\% (95\% Cl: 23.7- 27.2)) were tested positive while 296 herds (48.9\% (95\% Cl: 44.9-52.9)) had at least one positive animal (Table 3.2). The highest animal level apparent prevalence was recorded in Gozamn district followed by Sebeta Hawas and Jabitenan whereas the lowest prevalence was in Debrelibanos 
district (Table 3.2). The percentage of infected herds was highest in Ambo and lowest in Debrelibanos district (Table 3.2). Both animal and herd level prevalences varied significantly between the districts $(p<0.001)$. Kebele level seroprevalence distribution is presented in Figure 3.2.

The true overall animal level and herd level prevalences were estimated at 26.5\% (95\% Cl: $24.7-28.3$ ) and 51.0\% (95\% Cl: 46.8-55.1), respectively by considering $96 \%$ sensitivity and $100 \%$ specificity of VNT.

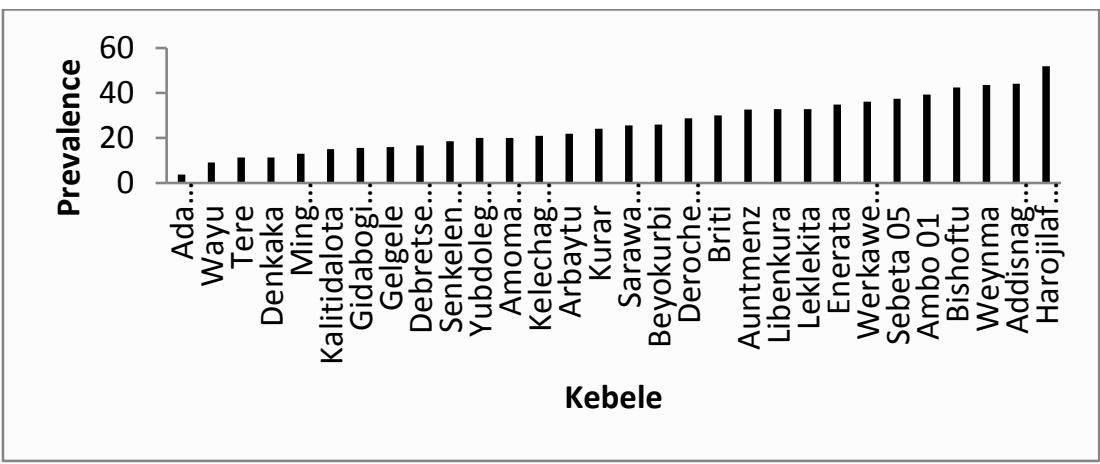

Figure 3.2. LSD seroprevalence of 30 kebeles in 10 districts of Ethiopia.

\subsubsection{Risk factors for LSD serostatus}

Results of the univariable mixed effect logistic regression are presented in Table 3.3. Accordingly, breed, age group, contact with other animals, free animal movement, and presence of water bodies were selected for the multivariable model $(p<0.25)$.

After removing the variable free animal movement due to collinearity with contact with other animals (VIF = 5.27 ), the final model showed age group $\geq 4$ years, OR: 2.44 (95\% Cl: 1.67-3.55), contact with other animals OR: 0.41 (95\% Cl: 0.23-0.74) and presence of water bodies OR: 1.61 (95\% Cl: 1.03-2.52) as significant factors associated with LSD status (Table 3.4).

\subsubsection{Intracluster correlation coefficient}

The inter-kebele variance was estimated as 0.382 (Table 3.4) resulting in an ICC of 0.104 (Formula 3). The design effect is then 9.2 and the precision reached for the unadjusted prevalence estimate equals of $2.7 \%$. 
Table 3.1. Descriptive information about cattle, kebeles and number of serum samples collected per district in a study to lumpy skin disease in Ethiopia.

\begin{tabular}{lcccc}
\hline Districts & $\begin{array}{c}\text { Cattle } \\
\text { pop. in } \\
\text { district }\end{array}$ & $\begin{array}{c}\text { No. of } \\
\text { kebeles in } \\
\text { district }\end{array}$ & $\begin{array}{c}\text { No. of } \\
\text { kebeles } \\
\text { sampled }\end{array}$ & $\begin{array}{c}\text { No. of serum } \\
\text { samples } \\
\text { collected }\end{array}$ \\
\hline Ada'a & 101,088 & 25 & 3 & 239 \\
Ambo & 370,000 & 35 & 3 & 245 \\
Debrelibanos & 59,760 & 11 & 3 & 244 \\
Dejen & 59,505 & 21 & 3 & 246 \\
Dendi & 212,357 & 49 & 3 & 242 \\
Gozamn & 185,799 & 25 & 3 & 235 \\
Hidabu Abote & 79,636 & 20 & 3 & 269 \\
Jabitenan & 237,677 & 37 & 3 & 209 \\
Kuyu & 140,400 & 23 & 3 & 218 \\
Sebeta Hawas & 151,400 & 39 & 3 & $\mathbf{2 3 8 6}$ \\
\hline Overall & $\mathbf{1 , 5 9 7 , 6 2 2}$ & $\mathbf{2 8 5}$ & $\mathbf{3 0}$ & \\
\hline
\end{tabular}




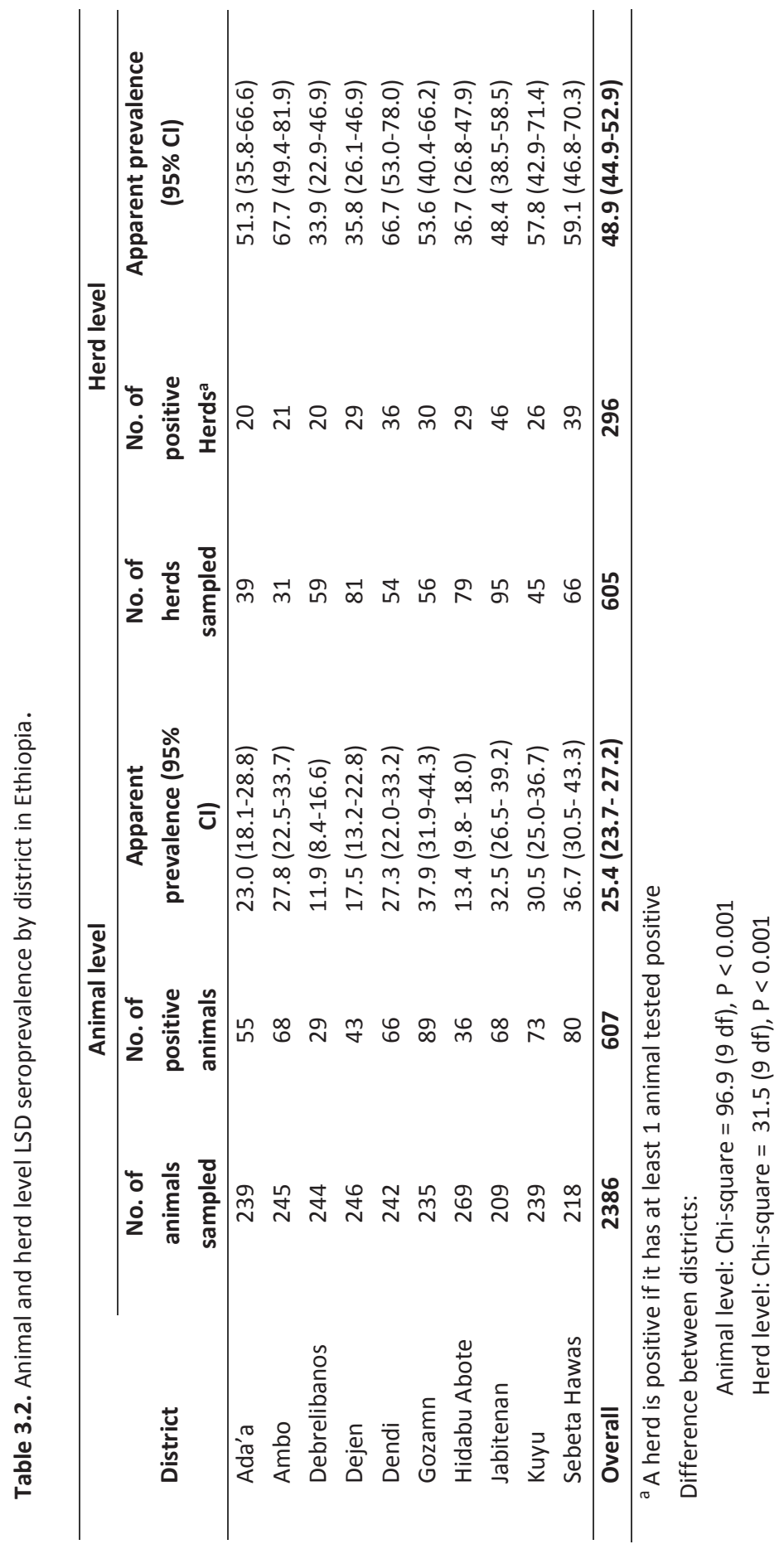




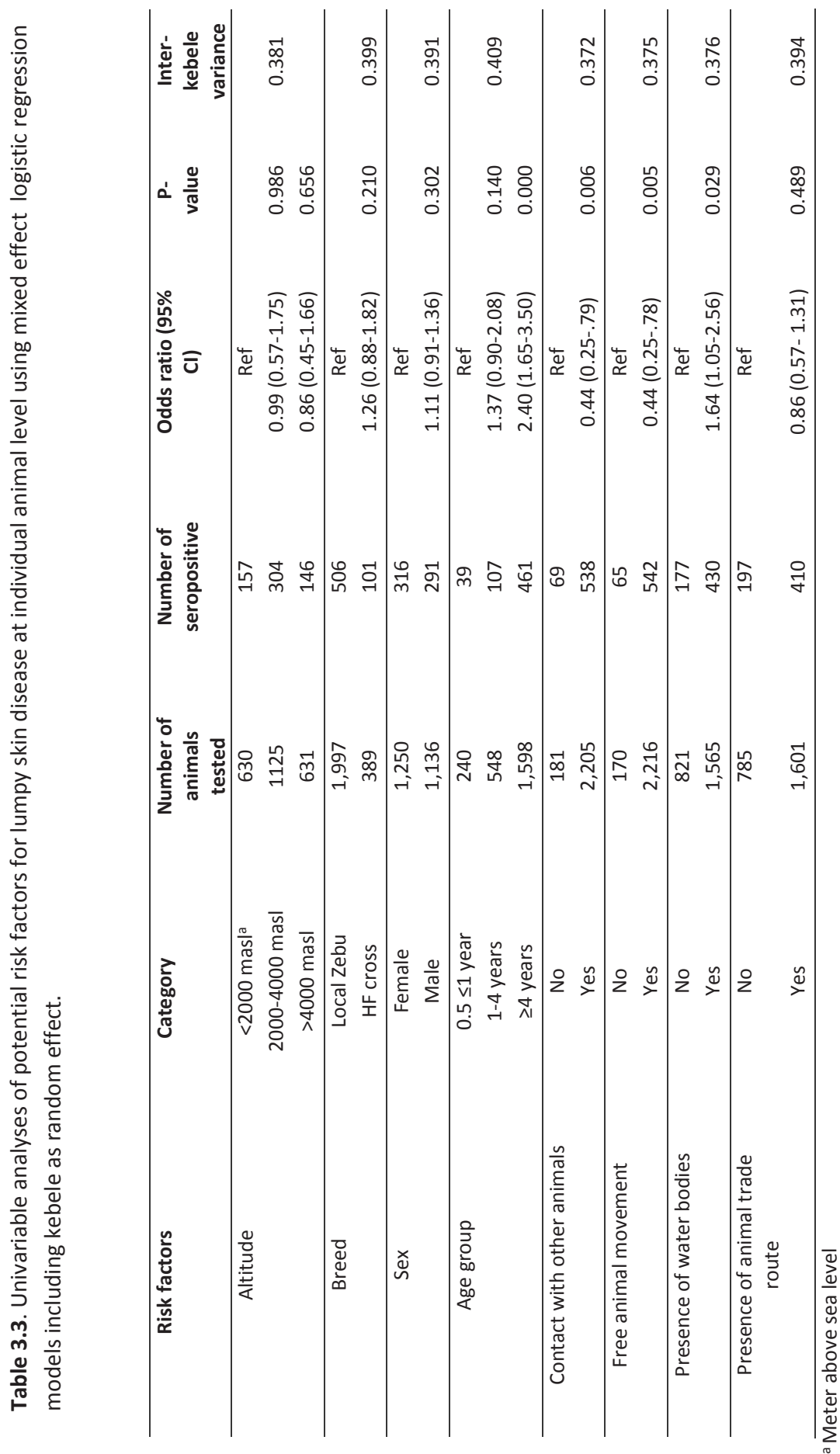




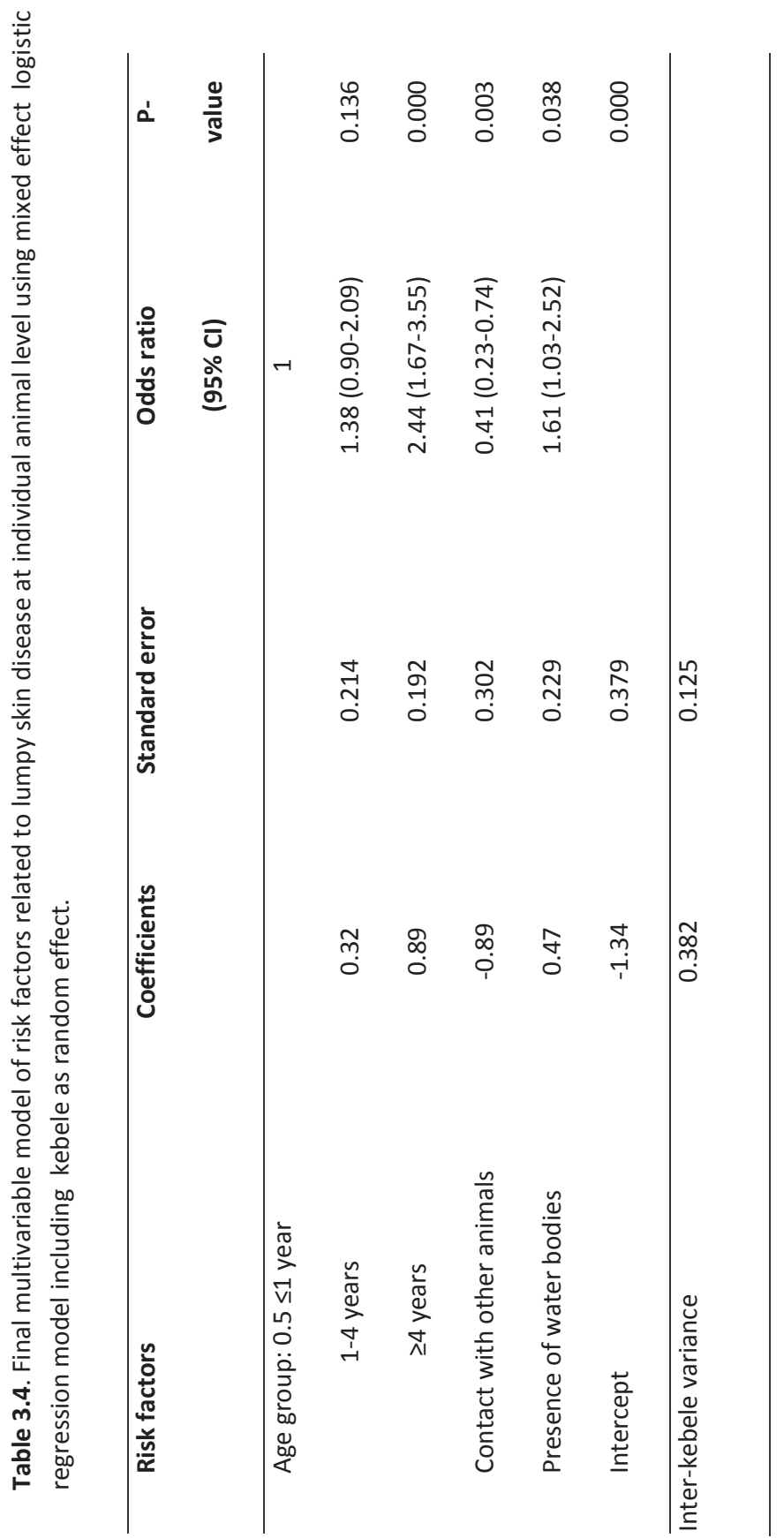




\subsection{Discussion}

In this cross-sectional study, the herd level prevalence was estimated at 48.9\% indicating that in half of the herds involved at least one LSD seropositive animal was present. This prevalence is close to the $44 \%$ herd level prevalence reported by Gari et al. (2012) and Hailu et al. (2014), but much higher than the $5.95 \%$ recorded by Abera et al. (2015). In the current study, $25.4 \%$ of the animals tested positive for LSDV antibody, leading to an estimated true prevalence of $26.5 \%$ in the target population. This is close to the seroprevalence of $27 \%$ reported by Gari et al. (2012), but considerably higher than the seroprevalence of $6.4 \%$ reported by Abera et al. (2015) in Western Wollega and the prevalence of $7.4 \%$ reported by Hailu et al. (2014) in a questionnaire based cross sectional study in north eastern Ethiopia. The difference in both herd and animal level prevalences might be due to the difference in efficiency and population densities of arthropod vectors, dissimilarity in the study area environment, a different study population, sampling period, and testing methods employed for the studies. The statistical methods used to compute the true prevalence might also contribute to the difference, though unadjusted apparent and true prevalences are close. In general, LSD seems to be endemic and widespread in mixed crop-livestock production area of Ethiopia, with all tested kebeles having at least one LSD positive animal.

Among the risk factors studied, age, contact with other animals, and presence of water bodies were significantly associated with LSD serostatus. Adult animals have higher odds to be affected $(O R=2.44)$ compared to calves (reference). This is in agreement with Abera et al. (2015) who recorded higher odds in adult (OR $=3.41$ ) and young $(O R=1.86)$ animals compared to calves. In Ethiopia, $63.5 \%$ of the cattle population is between 3-10 years of age and a large proportion of the animals is used for draft and milk production purposes (CSA, 2015). Therefore, the increased odds of being seropositive for young and adult animals might be attributed to stress and exhaustion related to lactation and heavy labour for milk and draft animals (Hunter and Wallace, 2001; OIE, 2010; Gari et al., 2011; Tuppurainen and Oura, 2012; Abera et al., 2015). On the other hand the low prevalence in calves might be related to the traditional calf management practices that segregate calves from the herd and this might lead to less exposure to vectors (Gari et al., 2011).

Results indicate that animals living in an area with surface water bodies (rivers, dams, lakes, ponds, irrigated and marshy areas) present have 1.61 times higher odds than animals living in areas with no such bodies. Presence of surface water increases the breeding success and survival of various vectors (McMichael, 2003) like for Aedes aegypti, which is seen as the vector for mechanical transmission 
of LSD (Chihota et al., 2001; Kalluri et al., 2007; Al-Salihi, 2014). The present finding supports previous works indicating that river deltas and basins, irrigated lands, and water courses create ideal conditions for arthropod vector populations that could enhance LSDV transmission (Woods, 1988; Davies, 1991; Hunter and Wallace, 2001; Tuppurainen and Oura, 2012). Epidemiologic observations also showed that LSD incidence is highest in wet/warm weather and lowest in the dry season, which is possibly linked to variation in vector population density (FAO, 2013).

Cattle in frequent contact with other animals and other animal species during extensive communal grazing and at watering places have two times lower odds to be infected by LSDV than animals herded separately. This is in contrast with other studies reporting that animals having a frequent contact with other animals at communal grazing and watering points are more at risk to acquire LSD (Gari et al., 2010; Hailu et al., 2014). In the study areas, several animal species (bovine, ovine, caprine, and equine) are allowed to graze and stay together on the same communal pasture land during day time. The inverse relationship we found may be attributed to the presence of alternative blood meal source hosts for the arthropod vectors. Keeping the competent hosts (cattle) together with non-competent hosts (sheep, goat, donkey, mule, horse) at the same pasture land may decrease vector infection/contamination rates and thus the transmission to cattle, a dilution effect (Swaddle and Calos, 2008; Randolph and Dobson, 2012).

In contrast to previous studies (Davies, 1991; Abera et al., 2015), breed did not play a significant role as risk factor for testing LSD positive. This could be explained by the distribution of the breeds among study areas. Most of the HF cross animals were in less affected districts such as Debrelibanos and Ada'a. The absence of significant association between altitude category and serostatus to LSD was observed in the current study unlike Gari et al. (2012) who reported a significantly higher animal level LSD seropositivity in the midland as compared to the highland and the lowland agro-climates, but it corroborates the findings of Abera et al. (2015). We did also find no association between sex and presence of trade routes with serostatus. This is in line with the previous reports by Gari et al. (2010) and Abera et al. (2015).

In developing countries like Ethiopia, information on disease prevalence is usually obtained by means of cross-sectional surveys. Because of unavailability of individual animal sampling frame and a high travel costs cluster sampling was preferred over simple random sampling (Otte and Gumm, 1997). Kebele was considered as a cluster and the intrakebele correlation coefficient (0.104) was medium indicating a certain degree of variation in LSDV seroprevalence between kebeles. This ICC value implies that the within cluster (between individual animal) 
variance is greater than the between cluster (kebele) variance. This finding is in line with the description that the estimate of ICC for most infectious diseases including LSD does not exceed 0.20 (Gari et al., 2012; Otte and Gumm, 1997). The design effect $(D=9.2)$ and the standard error ( $S E=0.027$ ) obtained indicate that the sample size used for the study was larger than what is needed to estimate the prevalence with a $5 \%$ precision and $95 \%$ confidence interval. The design effect appeared to be lower than anticipated and therefore the sample size could have been smaller.

\subsection{Conclusion}

The LSD seroprevalence is medium at animal level and high at herd level. Adult age, contact with other animals, and presence of water bodies are important factors for the occurrence of LSD. The within cluster variance is greater than the between cluster variance and the estimated LSD design effect is lower than anticipated. In general, this study revealed useful epidemiological information for designing plausible LSD control strategy plan in the country, which is non-existent at the moment. Areas endowed with rivers, lakes, ponds, dams, irrigated and swampy areas, which support the presence of abundant blood feeding arthropods, and cattle populations with many adult animals could be priority areas for application of control measures to reduce the burden of the disease.

\section{Acknowledgements}

The authors are grateful to NUFFIC (Netherlands Organization for International Cooperation in Higher Education) for funding the study. We would like to thank NAHDIC, NVI and Veterinary Services Directorate of Ethiopia for the material and logistic support and allowing to use their facilities. We also thank livestock owners and district animal health personnel for their cooperation and technical support during field work.

\section{References}

Abera, Z., Degefu, H., Gari, G., Kidane, M., 2015. Sero-prevalence of lumpy skin disease in selected districts of West Wollega zone, Ethiopia. BMC Vet. Res. $11,135$.

Al-Salihi, K.A., 2014. Lumpy Skin disease: Review of literature. MRSVA 3, 6-23.

Babiuk, S., Bowden, T.R., Boyle, D.B., Wallace, D.B., Kitching, R.P., 2008a. Capripoxviruses: an emerging worldwide threat to sheep, goats and cattle. Transbound. Emerg. Dis. 55, 263-272. 
Babiuk, S., Bowden, T.R., Parkyn, G., Dalman, B., Hoa, D.M., Long, N.T., Vu, P.P., Bieu, D.X., Copps, J., Boyle, D.B., 2009. Yemen and Vietnam capripoxviruses demonstrate a distinct host preference for goats compared with sheep. J. Gen. Virol. 90, 105-114.

Babiuk, S., Bowden, T.R., Parkyn, G., Dalman, B., Manning, L., Neufeld, J., EmburyHyatt, C., Copps, J., Boyle, D.B., 2008b. Quantification of lumpy skin disease virus following experimental infection in cattle. Transbound. Emerg. Dis. 55, 299-307.

Bennett, S., Woods, T., Liyanage, W.M., Smith, D.L., 1991. A simplified general method for cluster-sample surveys of health in developing countries. World Health Stat. Q. 44, 98-106.

Carn, V.M., Kitching, R.P., 1995. An investigation of possible routes of transmission of lumpy skin disease virus (Neethling). Epidemiol. Infect. 114, 219-226.

Chihota, C.M., Rennie, L.F., Kitching, R.P., Mellor, P.S., 2001. Mechanical transmission of lumpy skin disease virus by Aedes aegypti (Diptera: Culicidae). Epidemiol. Infect. 126, 317-321.

Chihota, C.M., Rennie, L.F., Kitching, R.P., Mellor, P.S., 2003. Attempted mechanical transmission of lumpy skin disease virus by biting insects. Med. Vet. Entomol. 17, 294-300.

CSA, 2015. Agricultural Sample Survey, 2014/15 (2007 E.C.), Volume II: Report on livestock and livestock characteristics (Private peasant holdings). Statistical Bulletin 578. Central Statistical Agency (CSA), Federal Democratic Republic of Ethiopia, Addis Ababa.

Davies, F.G., 1991. Lumpy skin disease, an African capripox virus disease of cattle. Br. Vet. J 147, 489-503.

Dohoo, I., Martin, W., Stryhn, H., 2012. Veterinary Epidemiologic Research AVC Inc Charlottetown, Canada.

FAO, 2013. Emergence of lumpy skin disease in the Eastern Mediterranean Basin countries. EMPRES WATCH, Rome, 1-6.

FAO, 2015. Emergence of lumpy skin disease (LSD) in Europe. 1-4.

Gari, G., Biteau-Coroller, F., LeGoff, C., Caufour, P., Roger, F., 2008. Evaluation of indirect fluorescent antibody test (IFAT) for the diagnosis and screening of lumpy skin disease using Bayesian method. Vet. Microbiol. 129, 269-280.

Gari, G., Bonnet, P., Roger, F., Waret-Szkuta, A., 2011. Epidemiological aspects and financial impact of lumpy skin disease in Ethiopia. Prev. Vet. Med. 102, 274283. 
Gari, G., Grosbois, V., Waret-Szkuta, A., Babiuk, S., Jacquiet, P., Roger, F., 2012. Lumpy skin disease in Ethiopia: seroprevalence study across different agroclimate zones. Acta Trop. 123, 101-106.

Gari, G., Waret-Szkuta, A., Grosbois, V., Jacquiet, P., Roger, F., 2010. Risk factors associated with observed clinical lumpy skin disease in Ethiopia. Epidemiol. Infect. 138, 1657-1666.

Hailu, B., Tolosa, T., Gari, G., Teklue, T., Beyene, B., 2014. Estimated prevalence and risk factors associated with clinical Lumpy skin disease in north-eastern Ethiopia. Prev. Vet. Med. 115, 64-68.

Hunter, P., Wallace, D., 2001. Lumpy skin disease in southern Africa: a review of the disease and aspects of control. J. S. Afr. Vet. Ass. 72, 68-71.

Kalluri, S., Gilruth, P., Rogers, D., Szczur, M., 2007. Surveillance of arthropod vectorborne infectious diseases using remote sensing techniques: a review. PLoS Pathog. 3, 1361-1371.

Lubinga, J.C., Tuppurainen, E.S., Mahlare, R., Coetzer, J.A., Stoltsz, W.H., Venter, E.H., 2015. Evidence of transstadial and mechanical transmission of lumpy skin disease virus by Amblyomma hebraeum ticks. Transbound. Emerg. Dis. 62, 174-182.

McMichael, A.J., 2003. Global climate change: will it affect vector-borne infectious diseases?. Intern. Med. J. 33, 554-555.

Mebratu, G.Y., Kassa, B., Fikre, Y., Berhanu, B., 1984. Observation on the outbreak of lumpy skin disease in Ethiopia. Rev. Elev. Méd. Vét. Pays Trop. 37, 395-399.

OIE, 2010. Manual of diagnostic tests and vaccines for terrestrial animals, chapter 2.4.14, Lumpy skin disease. OIE, Paris. http://web.oie.int/eng/normes/MMANUAL/A_Index.htm. Accessed 26 February 2016. OIE, Paris.

OIE, 2013. Lumpy skin disease. OIE Technical Disease Cards. OIE.

Otte, M.J., Gumm, I.D., 1997. Intra-cluster correlation coefficients of 20 infections calculated from the results of cluster-sample surveys. Prev. Vet. Med. 31, 147-150.

Quinn, P.J., Markey, B.K., Carter, M.E., Donnelly, W.J., Leonard, F.C., 2002. Veterinary Microbiology and Microbial Disease. Blackwell Science Great Britain. pp. 335-342.

Radostits, O.M., Gay, C.C., Hinchcliff, K.W., Constable, P.D., 2007. Veterinary Medicine: A Textbook of the Diseases of Cattle, Sheep, Pigs, Goats and Horses. Sounders Elsevier Spain. pp. 1424-1426 
Randolph, S.E., Dobson, A.D., 2012. Pangloss revisited: a critique of the dilution effect and the biodiversity-buffers-disease paradigm. Parasitology 139, 847863.

Rogan, W.J., Gladen, B., 1978. Estimating prevalence from the results of a screening test. Am. J. Epidemiol. 107, 71-76.

Swaddle, J.P., Calos, S.E., 2008. Increased avian diversity is associated with lower incidence of human West Nile infection: observation of the dilution effect. PloS one 3, e2488.

Tschopp, R., Schelling, E., Hattendorf, J., Aseffa, A., Zinsstag, J., 2009. Risk factors of bovine tuberculosis in cattle in rural livestock production systems of Ethiopia. Prev. Vet. Med. 89, 205-211.

Tuppurainen, E.S., Lubinga, J.C., Stoltsz, W.H., Troskie, M., Carpenter, S.T., Coetzer, J.A., Venter, E.H., Oura, C.A., 2013a. Evidence of vertical transmission of lumpy skin disease virus in Rhipicephalus decoloratus ticks. Ticks Tick-borne Dis. 4, 329-333.

Tuppurainen, E.S., Lubinga, J.C., Stoltsz, W.H., Troskie, M., Carpenter, S.T., Coetzer, J.A., Venter, E.H., Oura, C.A., 2013b. Mechanical transmission of lumpy skin disease virus by Rhipicephalus appendiculatus male ticks. Epidemiol. Infect. 141, 425-430.

Tuppurainen, E.S., Oura, C.A., 2012. Review: lumpy skin disease: an emerging threat to Europe, the Middle East and Asia. Transbound. Emerg. Dis. 59, 40-48.

Tuppurainen, E.S., Stoltsz, W.H., Troskie, M., Wallace, D.B., Oura, C.A., Mellor, P.S., Coetzer, J.A., Venter, E.H., 2011. A potential role for ixodid (hard) tick vectors in the transmission of lumpy skin disease virus in cattle. Transbound. Emerg. Dis. 58, 93-104.

Tuppurainen, E.S., Venter, E.H., Shisler, J.L., Gari, G., Mekonnen, G.A., Juleff, N., Lyons, N.A., De Clercq, K., Upton, C., Bowden, T.R., Babiuk, S., Babiuk, L.A., 2017. Review: capripoxvirus diseases: current status and opportunities for control. Transbound. Emerg. Dis. 64 729-745.

Woods, J.A., 1988. Lumpy skin disease- A review. Trop. Anim. Health Prod. 20, 11-17.

Zeynalova, S., Asadov, K., Guliyev, F., Vatani, M., Aliyev, V., 2016. Epizootology and molecular diagnosis of lumpy skin disease among livestock in Azerbaijan. Frontiers Microbiol. 7, 1022. 


\title{
4
}

\section{Transmission dynamics of lumpy skin disease in Ethiopia}

\author{
W. Molla ${ }^{1,2}$, K. Frankena ${ }^{1}$, M.C.M. de Jong ${ }^{1}$ \\ ${ }^{1}$ Quantitative Veterinary Epidemiology, Wageningen University \& Research, \\ Droevendaalsesteeg 1, 6708 PB Wageningen, The Netherlands \\ ${ }^{2}$ Faculty of Veterinary Medicine, University of Gondar, P.O. Box 196, Gondar, \\ Ethiopia
}

Epidemiology and Infection (2017) 145: 2856-2863

DOI: $10.1017 /$ S0950268817001637 


\begin{abstract}
Lumpy skin disease (LSD) is a severe disease of cattle caused by a Capripoxvirus and often caused epidemics in Ethiopia and many other countries. This study was undertaken to quantify the transmission between animals and to estimate the infection reproduction ratio in a predominantly mixed crop-livestock system and in intensive commercial herd types. The transmission parameters were based on a SIR epidemic model with environmental transmission and estimated using generalized linear models. The transmission parameters were estimated using a survival rate of infectious virus in the environment equal to 0.325 per day, a value based on the best fitting statistical model. The transmission rate parameter between animals was 0.072 (95\% Cl: 0.068-0.076) per day in the crop-livestock production system, whereas this transmission rate in intensive production system was 0.076 (95\% Cl: 0.068-0.085) per day. The reproduction ratio (R) of LSD between animals in the crop-livestock production system was 1.07, whereas it was 1.09 between animals in the intensive production system. The calculated $\mathrm{R}$ provides a baseline against which various control options can be assessed for efficacy.
\end{abstract}

Key words: Cattle, Ethiopia, LSD, Transmission, Reproduction ratio 


\subsection{Introduction}

Lumpy skin disease (LSD) is a severe viral disease of cattle, which often occurs as regional epidemics within a larger area in which it is endemic. It is caused by Lumpy skin disease virus (LSDV) which is of the genus Capripoxvirus of family Poxviridae. LSDV is one of the most important animal poxviruses because of the serious economic consequences in cattle (Davies, 1991; Carn, 1993). The disease is characterized by lachrymation, fever, nodular lesions on the skin and mucosal surfaces, lymph node enlargement, inflammatory and oedematous swelling of the legs and lameness (Davies, 1991; Tuppurainen and Oura, 2012).

The disease was reported for the first time in Zambia in 1929 and was confined to Africa until an outbreak occurred in Israel in 1989 (Davies, 1991). However, currently, the disease is found in most African and Middle East countries and recently it has spread to eastern and south eastern European countries. LSDV is clearly on the move in expanding its territory and increasingly becoming a risk for other Asian and European countries (Tuppurainen et al., 2015).

Though the mechanism of LSDV transmission has not yet been clearly established, it is hypothesized that the main mode of transmission of LSDV is via blood feeding arthropods (Carn and Kitching, 1995). Experimentally, female Aedes aegypti mosquitoes have been shown to transmit LSDV mechanically from infected to susceptible cattle (Chihota et al., 2001). The potential role of ixodid ticks in transmission of LSDV has also been demonstrated in transmission studies including mechanical transmission between cattle for Amblyomma hebraeum and Rhipicephalus appendiculatus, trans-stadial transmission for A. hebraeum, and transovarial transmission for Rhipicephalus (Boophilus) decoloratus (Tuppurainen et al., 2011; Lubinga et al., 2013; Tuppurainen et al., 2013b, a; Lubinga et al., 2014). Transmission of LSDV between infected and susceptible animals by direct contact is considered to be inefficient (Carn and Kitching, 1995; Magori-Cohen et al., 2012).

Data from infectious disease outbreaks are usually incomplete and highly dependent. Incomplete because the infection process is only partially observable, i.e. not all cases may be included due to under-reporting or because of asymptomatic cases, the number of susceptible animals may not be known exactly, individuals who enter or leave the study population may not be recorded accurately, there may be misdiagnosis of cases and flaws in data collection. Data such as daily or weekly case 
numbers are obviously dependent (O’Neill, 2010 ; Becker and Britton, 1999). However, transmission under field conditions can be estimated from the number of infections that occurred during the study period or at certain intervals by mathematical modelling using exactly that dependence (Kroese and De Jong, 2001; Velthuis et al., 2002).

One parameter often used to characterize transmission is the basic reproduction ratio $\left(R_{0}\right)$ with the effective reproduction ratio $\left(R_{e}\right)$ being the parameter for the transmission after intervention. $R_{0}$ is defined as the average number of secondary infections caused by one typical infectious individual in a fully susceptible population during its entire infectious period (Diekmann et al., 1990), whereas $R_{e}$ reflects the transmission parameter in a partially susceptible (previously exposed or vaccinated) population (Chowell and Nishiura, 2014). The reproduction ratio (R) is frequently used to describe the behaviour of transmission just after introduction of a disease. Whether an outbreak spreads or dies out depends on whether the reproduction ratio is greater than, or less than, 1 respectively. If $R$ exceeds 1 , a typical (i.e. average) infected animal infects on average more than one susceptible animal, and thus it may cause a major outbreak, while if $R$ is smaller than 1 the disease will die out or it will at most produce a minor outbreak (Velthuis et al., 2002; van Roermund et al., 2010 ).

Despite a large number of LSD outbreaks in many African and Middle East countries, its dynamics are not well studied. Only one study, undertaken by MagoriCohen et al. (2012) in a dairy herd of Israel, reports an estimate for the reproduction ratio of LSDV $\left(R_{0}=15.7\right)$. Therefore, the current study was undertaken with the objectives to better understand the LSDV outbreak dynamics and to quantify the transmission rate parameter and the reproduction ratio between animals.

\subsection{Materials and methods}

\subsubsection{Study area, farms and animals contact patterns}

The study was carried out from 28 April 2014 to 1 February 2015 in the central and north-western parts of Ethiopia. In the north-western part, it involves the cattle population in Mota town and parts of the surrounding five Kebeles (Kebele is the smallest administrative unit in Ethiopia covering an approximate area of $53 \mathrm{~km}^{2}$ ) in Hulet Ejju Enessie district, and Debremarkos University dairy farm in Gozamn district. In the central part, the following herds were enrolled: Selale Dairy Development 
Private Limited Company (Selale Dairy Dev't PLC) in Wuchale district, Aser Dev't PLC in Sululta district, Ambo University dairy farm in Ambo district, Holeta agricultural research centre farm (Holeta A.R.C) and Holeta special cattle breeding centre (Holeta S.C.B.C) in Welmera district, Selam children village dairy farm in Addis Ababa and Jenesis dairy farm in Ada'a district (Figure 4.1). Mota area (Mota town and parts of the surrounding five Kebeles) covers an area of about five $\mathrm{km}$ radius. The production system in the Mota area is mainly mixed crop-livestock while the other herds were commercial dairy herds. Most of the animals in the mixed crop-livestock type of herds were of local Zebu breed whilst the intensive herds consisted of Holstein-Zebu cross. Farms were categorized into small (<10 cattle), medium (10-50 cattle), large (51-300 cattle), very large (301-700 cattle) and extra-large ( $>700$ cattle) based on the number of cattle they comprised.

The cattle contact network depends on a number of factors including housing system, size and nature of grazing lands, water points, cattle density, and frequency and duration of contacts. This study was undertaken at the family herd (group of animals owned by a family for subsistence) and commercial farm (group of animals owned by a private or public organization for commercial purpose) levels. All smallholder herds enrolled in the study were in the Mota area, but the intensive commercial farms were located in different areas. Since the smallholder herds in the subsistence crop-livestock system (Mota area) are managed extensively, they regularly mixed at shared pastures and watering points so that they had to be considered as one epidemiological unit. Animals in the intensive commercial farms, however, did not have direct contact with animals in other farms in their surroundings and most of them were located in districts far apart from each other. 


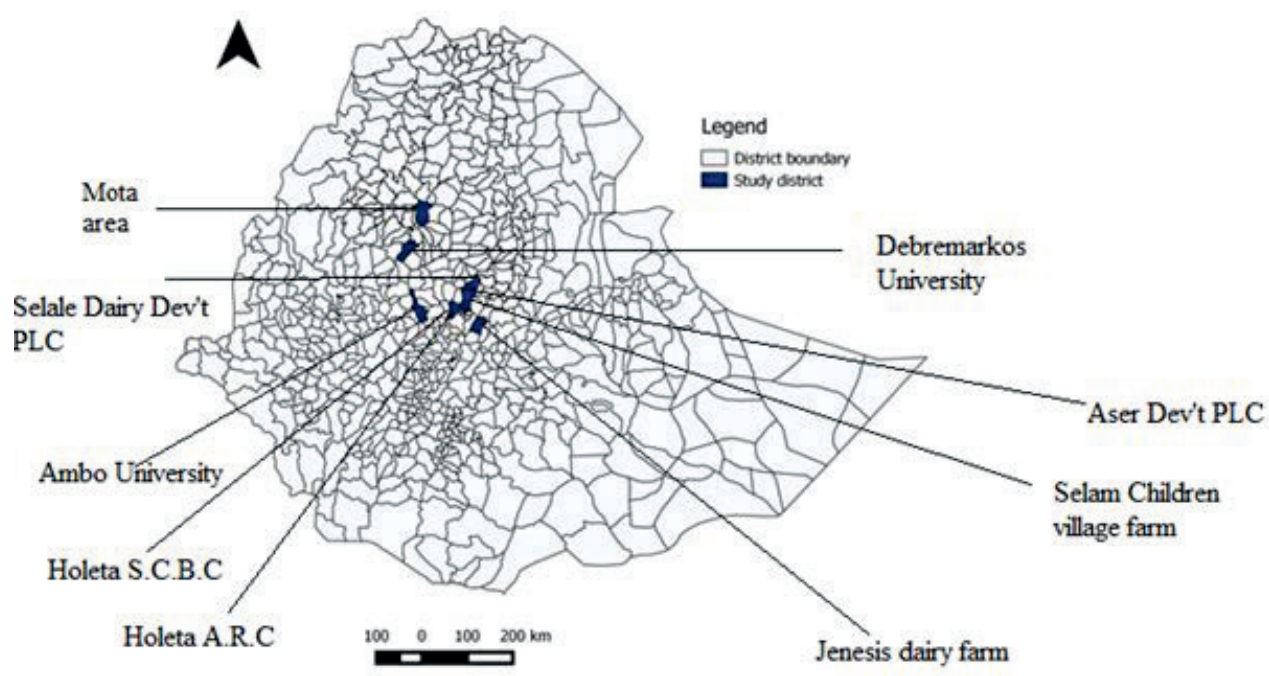

Figure 4.1. Map of Ethiopia showing LSD transmission study districts.

\subsubsection{Period of the epidemic}

To assess the association between LSD epidemics and the season of the outbreak (which has a strong relation with arthropod dynamics), the outbreak duration was categorized into three periods, Belg (period 1), Kiremt (period 2) and Bega (period 3) following the meteorological seasons of Ethiopia. Belg is a short rainy period from February to May over much of the Belg-growing areas. However, over the north-western parts of the country (where Mota area is located) this season is predominantly dry except for the month of May. Kiremt is the period from June to September; it is the main rainy season in which the major food crops of the country are produced. The magnitude of rainfall during Kiremt is higher as compared to the other seasons for many parts of the country. Bega is the period from October to January. It is normally a dry season characterized by cool nights and hot days over various parts of the country (NMA, 2013).

\subsubsection{Infection status of animals}

Herds were visited every week to check whether or not animals showing symptoms of LSD were present. If so, the infection chain within the herd was monitored by visiting the affected herd twice a week throughout the study period 
and the LSD status (susceptible, infected or recovered) of all animals was determined. At the start of the study all cattle were assumed to be susceptible. The start of the infectious period was considered to be the day following that on which an animal was first reported with clinical signs of LSD. Infected animals were assumed to stay infectious on average for 10 days taking the duration of viraemia as a proxy for period of infectivity (Woods, 1990; Carn and Kitching, 1995; Tuppurainen et al., 2005). An infected animal becomes most infective during the viraemic phase of the disease because the amount of virus in various body tissues and secretions and excretions of the animal become the highest in this phase (Woods, 1990). Animals that died before the infectious period was completed were considered infectious for the days they lived after being considered infectious.

The contribution of environment (E) to the transmission of LSDV was established by determining a per day survival rate of LSD virus shed into the environment by infected animals. This was done by fitting a GLM model to the collected data by varying the survival rate from 0.1 to 0.9 and selecting the best fitting model with the lowest AIC value.

Nodular samples were collected from few affected cattle in each herd to confirm the outbreak by using conventional and snapback real-time PCR (polymerase chain reaction) techniques following the procedure described by Gelaye et al. (2013).

\subsubsection{Estimation of the transmission parameters}

The transmission parameters were estimated based on a SIR epidemic model in which individuals are either susceptible (S), infected and infectious (I) or recovered and immune or dead (R). During the study, the numbers of I and S observed in each herd were recorded at the start of each observation interval. Animals were registered as a new case (C) on the date they were reported with LSD and as infectious (I) on the next day. Transmission of LSDV between animals has been estimated from the relationship between the number of infectious animals at the start of the time interval and the number of newly infected animals at the end of the time interval. Every new infection is related to the number of animals that were infectious at the time of infection.

The transmission parameters were estimated by a generalized linear model (GLM) (Velthuis et al., 2003; Heffernan et al., 2005; Chowell et al., 2007; Bravo de 
Rueda et al., 2015). The transmission dynamics of LSD between individuals are described by the change in the number of susceptible (S), infectious (I), and recovered (R) animals. Susceptible cattle become infected with a rate of $\beta \cdot S_{t} \cdot\left(I_{t}+E_{t}\right) / N_{t}$. Here, $\beta$ is the transmission rate which can be interpreted as the average number of new infections caused by a typical infectious animal in a fully susceptible population per unit of time, $S_{t}$ is the number of susceptible animals, $I_{t}$ the number of infectious animals, $E_{t}$ contribution of the environment to the transmission, and $\mathrm{N}_{\mathrm{t}}$ is the total number of animals at time $\mathrm{t}$, and they are assessed at the start of each observation period. The number of infectious contacts encountered by one individual in a period of length $\Delta t$ follows a Poisson distribution with parameter $\left(\beta \cdot\left(\mathrm{I}_{\mathrm{t}}+\mathrm{E}_{\mathrm{t}}\right) / \mathrm{N}_{\mathrm{t}} \cdot \Delta t\right)$. Hence, the probability of a susceptible animal escaping infection, during a period $\Delta t$ is $e^{-\beta \cdot \Delta t \cdot\left(l_{t}+E_{t}\right) / N_{t}}$, and thus the probability to become infected is $1-e^{-\beta \cdot \Delta t \cdot\left(l_{t}+E_{t}\right) / N_{t}}$. This implies that the number of new cases (C) in a period $\Delta t$ follows a binomial distribution. Consequently, the relation between the expected number of cases per unit of time $E(C)$, and $I_{t}, E_{t}, N_{t}, \beta$, and $S_{t}$ can be formulated as $E\left(C_{t}\right)=S_{t} \cdot\left(1-e^{-\beta \cdot \Delta t \cdot\left(I_{t}+E_{t}\right) / N_{t}}\right)$. The transmission parameter $\beta\left(\beta=e^{b}\right.$, where $b$ is the regression coefficient of the intercept of the model) was estimated using a GLM with a complementary-log-log link function and $\log \left(\Delta \mathrm{t} \cdot \frac{\mathrm{l}_{\mathrm{t}}+\mathrm{E}_{\mathrm{t}}}{\mathrm{N}_{\mathrm{t}}}\right)$ as offset. Finally, we obtained $R$ by multiplying $\beta$ with the average length of the infectious period (Velthuis et al., 2003; van Roermund et al., 2010; Bravo de Rueda et al., 2015) times a factor of $(1-E)^{-1}$ which incorporates the environmental contribution.

The Chi-square test was used to test the association of morbidity and mortality with production systems and GLM to compare transmission rates between the three meteorological periods, production systems and herd sizes.

All analyses were carried out in Stata 14.

\subsection{Results}

\subsubsection{Descriptive statistics}

During the study period, a total of 14,319 individual animals from 2,446 herds were followed for LSD occurrence. 12,509 animals (in 2,438 herds) were kept in the crop-livestock system and 1,810 animals (in 8 herds) in the intensive production system (Table 4.1).

The number of animals and herds affected, morbidity and mortality due to LSD per production system are indicated in Table 4.1. The morbidity was significantly higher in the intensive (17.5\%) compared to the crop-livestock $(10.1 \%)$ system. The 
mortality was also significantly higher in the intensive (4.0\%) than in the croplivestock (0.7\%) system (Table 4.1$)$.

In the Mota area, the LSD outbreak started at the end of April 2014 but in the other study farms the outbreak started later and continued until the first week of February 2015. The epidemic curve of the LSD outbreak in the Mota area is presented in Figure 4.2.

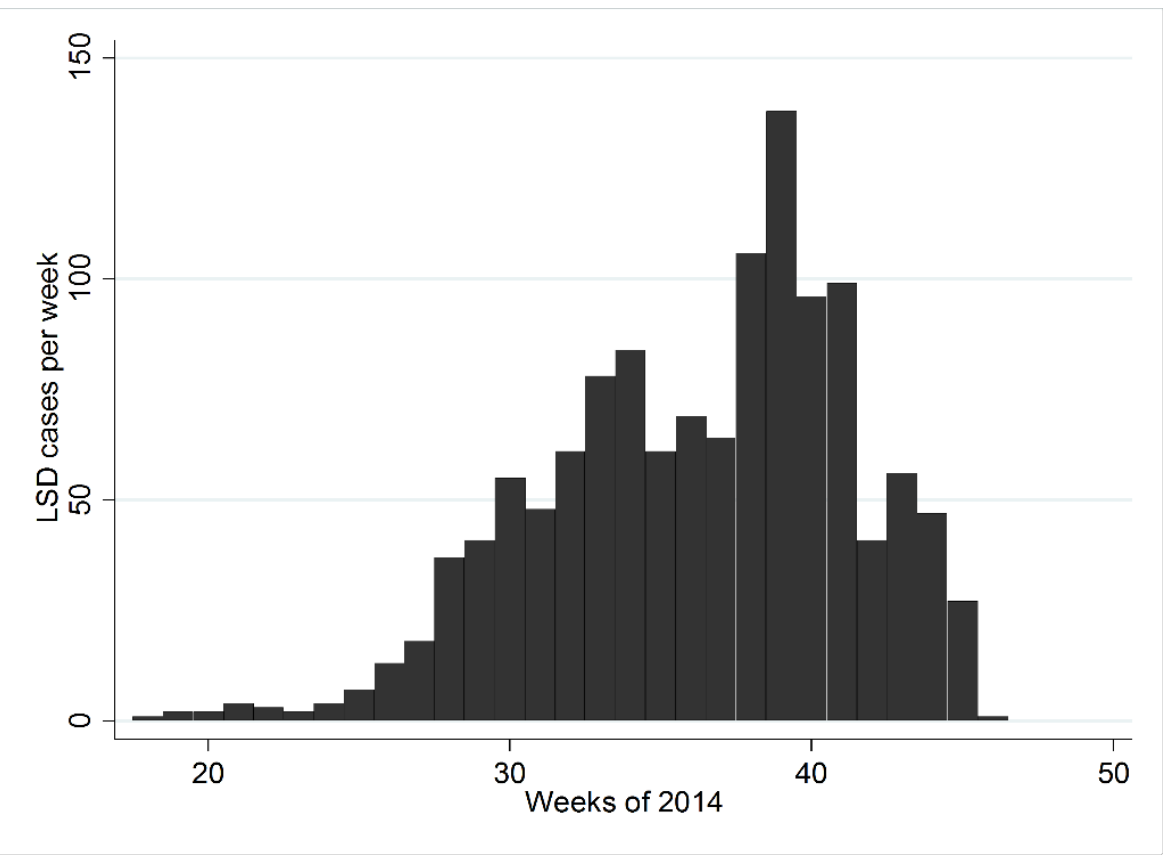

Figure 4.2. Epidemic curve of lumpy skin disease in Mota area, Ethiopia, in 2014. 


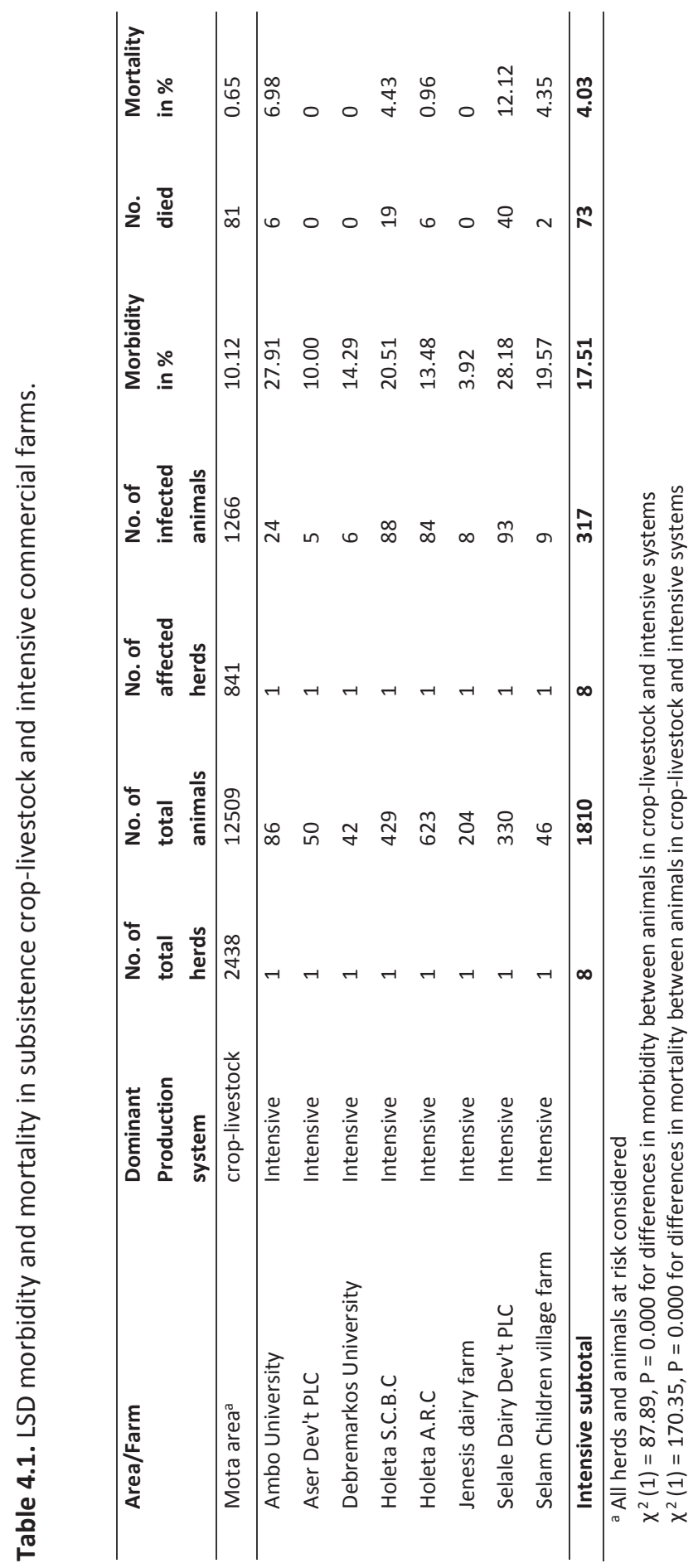




\subsubsection{Transmission of LSD between animals}

The contribution of the environment to the transmission (E) and the number of C, I and S animals in the Mota area are listed for each day of the epidemic (Appendix 4.1). The transmission rate parameter between animals in the dominantly subsistent crop-livestock production system was 0.072 (95\% Cl: 0.068-0.076) per day (Table 4.2) whereas in the intensive production system it was 0.076 (0.068-0.085) per day (Table 4.3). The survival rate of infectious LSD virus in the environment was estimated as 0.325 per day based on the best fitting statistical model and this value was used to account for the indirect transmission (excluding the immediate or direct transmission) of the virus. The average LSD infectious periods for animals are indicated in Table 4.2 and 4.3 for both production systems.

Based on the survival rate of LSDV in the environment, the multiplication factor of $\mathrm{R}$ was 1.5. Then a reproduction ratio of 1.07 between animals was calculated in the crop-livestock production system in the Mota area (Table 4.2). $R$ values between animals vary from 0.90 (Aser dairy farm) to 1.15 (Ambo university) in the eight intensive farms while the overall $R$ value for intensive dairy farms was 1.09 (Table 4.3). Major outbreaks have been observed in Ambo University, Holeta S.C.B.C, Holeta A.R.C, Selale Dairy Dev't PLC, Selam children village dairy herds and Mota area (Table 4.3, Appendix 4.2).

Transmission parameter rates $(\beta)$ between animals for subsistence croplivestock production system in the Mota area showed significant differences between period two and three $(P<0.05)$ (Table 2$)$. However, the transmission rates did not significantly differ between production systems and herd sizes. 


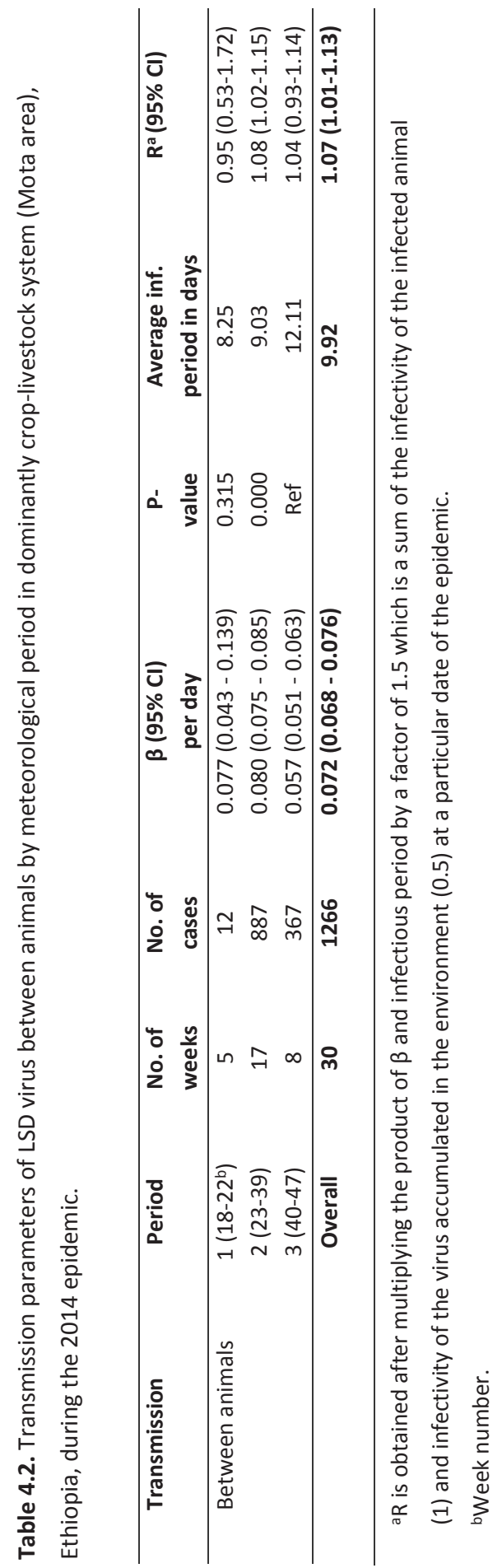




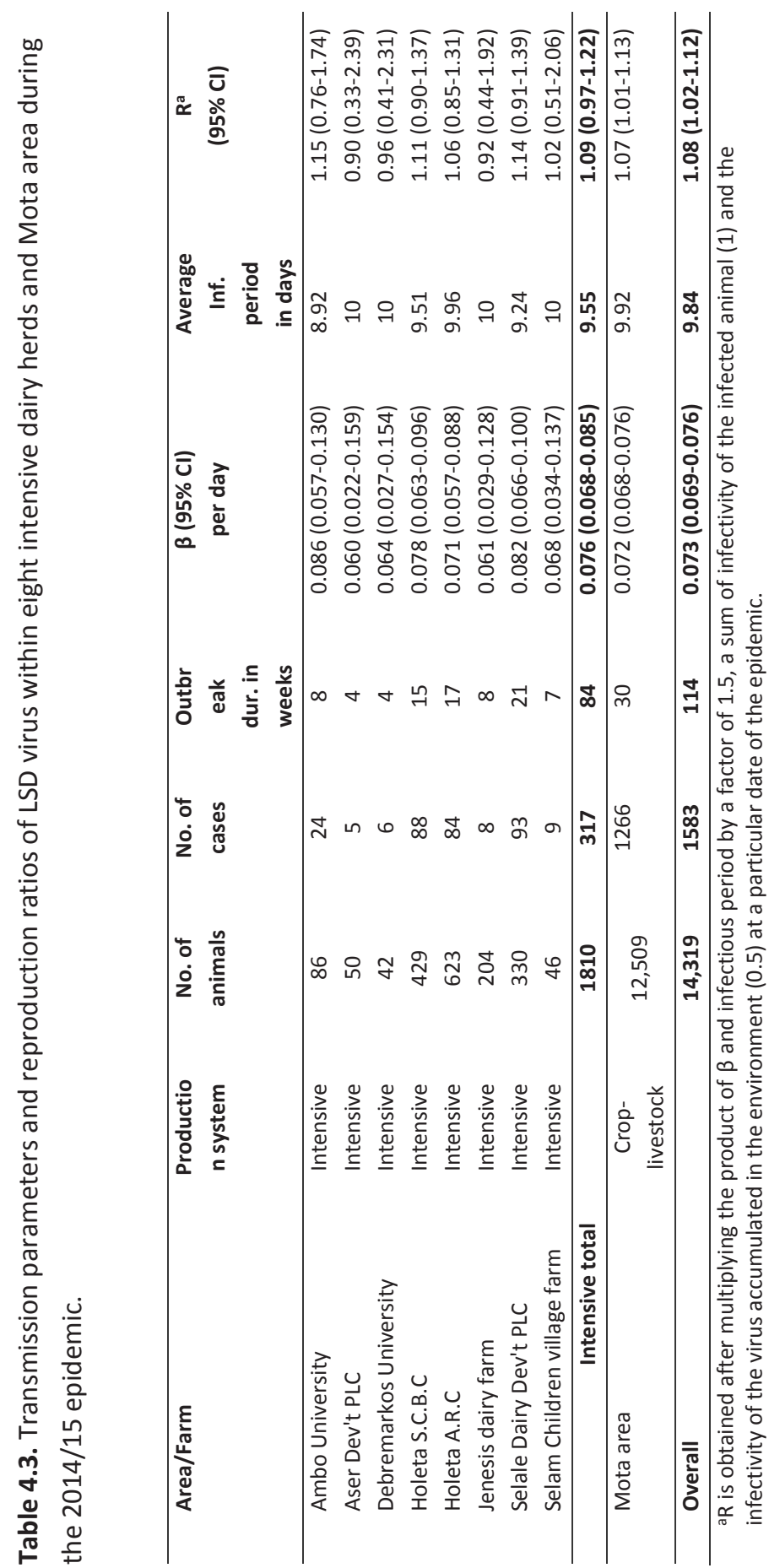




\subsection{Discussion}

The $10.1 \%$ and $17.5 \%$ animal level morbidity of LSD reported in the current study in the subsistence crop-livestock production system and intensive system, respectively, are within the range of what has been reported in previous works (Woods, 1988; Davies, 1991). Similarly, the mortality was higher in the intensive production system than in the crop-livestock system. These significant differences in morbidity and mortality between animals in the two systems might be explained by the breed of cattle raised in the two systems. In the intensive system, HolsteinFriesian local cross was the dominant breed which is more susceptible and more severely affected by LSD than the local Zebu breed (Davies, 1991; OIE, 2010), which is the breed commonly found in the crop-livestock production system. The other reason might be related to the way we calculated the morbidity and mortality in both systems. In the crop-livestock system, all animals in the Mota area whether or not they were within an infected herd or not, were included in the denominator, whereas in the intensive system only the number of animals in infected herds were in the denominator to calculate the morbidity and mortality.

The infectious period and survival of the virus in the environment are important parameters in estimating the reproduction ratio but these parameters were not reported in any of the previous studies. However, information about these parameters is essential for formulating appropriate prevention and control strategies for LSD. In this study too we did not estimate the infectious period of an infected animal and the survival rate of the virus in the environment because the study set up did not allow us to do that; instead we parametrized the infectious period from information obtained in the literature and the survival rate by searching for the best fitting model. We set the infectious period to 10 days for an infected animal by taking into account the duration of virus isolation in blood for 10-12 days (Carn and Kitching, 1995; Tuppurainen et al., 2005). Furthermore, there is no clear information when infected animals become infectious, which is important to know for the quantification of transmission. Infectiousness may start before or after the onset of clinical disease, but for this study we set the start of the infectious period as $24 \mathrm{~h}$ after the onset of the disease considering that LSDV isolation from blood and skin samples were achieved in most of the cases after the affected animals showed fever (Tuppurainen et al., 2005). Regarding the survival rate of the virus in the environment, literature indicates that the virus survives in air-dried hides for at least 18 days, in necrotic skin nodules for up to 33 days or longer, and for up to 35 days in 
desiccated crust (OIE, 2013), but it is not clear whether the viruses surviving in these foci contribute to the transmission of LSD. Taking this information into consideration we fitted a model (by selecting the best fitting model) to our data and found a survival rate of 0.325 per day, which was used in the offset to incorporate the contribution of environment to the transmission of LSDV. The implication of this survival rate is that the infectivity is increased by almost $50 \%$.

To our knowledge, this is the first field study in Ethiopia in which transmission rate parameters have been quantified. This knowledge is helpful to design sets of measures that efficiently eliminate the virus. In the study, LSDV transmission was modelled by considering it as direct transmission. It is widely believed that LSDV is transmitted from infected to susceptible hosts indirectly through mechanical arthropod vectors, though the importance of the different types of arthropod vectors in the transmission of LSD virus in field conditions is not fully understood (Carn and Kitching, 1995; Magori-Cohen et al., 2012). If a blood feeding arthropod feeds briefly on viraemic cattle and is interrupted, a subsequent immediate feeding on a second animal could result in virus transmission. The virus does not replicate within the vector (Goddard, 2008) which thus serves as a passive carrier to transmit the disease. The vector in this case serves only as a bridge for the transmission of LSDV from infected to susceptible cattle so that we did not incorporate the vectors in the transmission model.

During the outbreak, LSDV was transmitted between animals with a rate of 0.072 per day in the crop-livestock production system. The transmission chain from which specific infected cattle to which susceptible cattle was not clearly identified due to the free movement and mixing up of animals in the area and mechanical transmission of the disease by arthropods vectors. Hence, the transmission rate between animals was calculated by considering the cattle population in the area as one population.

In the Mota area, the transmission rate of LSD was also estimated for different time periods and the results indicate a significant difference in daily transmission rates between periods. The per day transmission rate between animals was higher at the beginning of the outbreak (in period 1 and 2 compared to period 3 ). This was expected, because during these periods the susceptible population was not yet depleted and no specific measures were taken to reduce transmission. This result 
indicates that starting implementation of control measures at early stage of the outbreak is necessary to halt the spread of the disease. We did not assess the periodic variation of transmission rate in farms of intensive production system due to the fact that the outbreaks in those farms were relatively short and it was not convenient to divide the time into different periods as in most occasions the outbreak fell in one period.

In this study, we estimated an $\mathrm{R}$ value of 1.07 between animals in the croplivestock area. The $R$ values within the intensive farms were also in the range of 0.90 to 1.15 with an overall value of 1.09 . These $R$ values are low compared with the $R$ value of 15.7 reported for indirect transmission within a commercial dairy farm in Israel (Magori-Cohen et al., 2012). The difference might be explained by the method how $\mathrm{R}$ is calculated, different study population, the environmental difference and the production set up.

Knowledge of within herd transmission is necessary to assess the effectiveness of intervention measures and to design effective monitoring programmes (Stegeman et al., 1999; Graat et al., 2001; Hage et al., 2003 ). In this study, we estimated that $\mathrm{R}$ was greater than 1 between animals in the dominantly crop-livestock system and in some farms of the intensive production system. This sheds light on LSDV transmission and further work should focus on the effect of control measures that add to bring $R$ below the threshold level. LSD control will be achieved if both reproduction ratios, among animals and between herds are less than 1; and also if $R$ among animals is greater than 1 , but $R$, between herds is below 1. Infections with low $R$ values are less difficult to control than those with a high $R$ value (Hage et al., 2003 ). Our estimates of $R$ provides a baseline against which various control options can be assessed for efficacy. In general, from this study it can be concluded that transmission of LSDV between animals in Ethiopia is low.

\section{Acknowledgments}

The authors are grateful to The Netherlands Organization for International Cooperation in Higher Education(NUFFIC) for financing this study. We would also like to thank animal health personnel and the herd owners for their kind collaboration in collecting and providing information for the study. 


\section{References}

Becker, N.G., Britton, T., 1999. Statistical studies of infectious disease incidence J. R. Statist. Soc. B 61, 287-307.

Bravo de Rueda, C., de Jong, M.C.M., Eblé, P.L., Dekker, A., 2015. Quantification of transmission of foot-and-mouth disease virus caused by an environment contaminated with secretions and excretions from infected calves. Vet. Res. 46. doi: 10.1186/s13567-015-0156-5.

Carn, 1993. Control of capripoxvirus infections. Vaccine 11, 1275-1279.

Carn, V.M., Kitching, R.P., 1995. An investigation of possible routes of transmission of lumpy skin disease virus (Neethling). Epidemiol. Infect. 114, 219-226.

Chihota, C.M., Rennie, L.F., Kitching, R.P., Mellor, P.S., 2001. Mechanical transmission of lumpy skin disease virus by Aedes aegypti (Diptera: Culicidae). Epidemiol. Infect. 126, 317-321.

Chowell, G., Nishiura, H., 2014. Transmission dynamics and control of Ebola virus disease (EVD): a review. BMC Medicine 12.

Chowell, G., Nishiura, H., Bettencourt, L.M., 2007. Comparative estimation of the reproduction number for pandemic influenza from daily case notification data. J. R. Soc. Interface 4, 155-166.

Davies, F.G., 1991. Lumpy skin disease, an African capripox virus disease of cattle. Br. Vet. J. 147, 489-503.

Diekmann, O., Heesterbeek, J.A.P., Metz, J.A.J., 1990. On the definition and the computation of the basic reproduction ratio $\mathrm{RO}$ in models for infectious diseases in heterogeneous populations J. Math. Biol. 28, 365-382.

Gelaye, E., Lamien, C.E., Silber, R., Tuppurainen, E.S., Grabherr, R., Diallo, A., 2013. Development of a cost-effective method for capripoxvirus genotyping using snapback primer and dsDNA intercalating dye. PloS one 8, e75971.

Goddard, J., 2008. Infectious Diseases and Arthropods. Humana Press Totowa NJ, USA.

Graat, E.A.M., de Jong, M.C.M., Frankena, K., Franken, P., 2001. Modelling the effect of surveillance programmes on spread of bovine herpesvirus 1 between certified cattle herds. Vet. Microbiol. 79, 193-208.

Hage, J.J., Schukken, Y.H., Schols, H., Maris-Veldhuis, M.A., Rijsewijk, F.A.M., Klaassen, C.H.L., 2003. Transmission of bovine herpesvirus 1 within and between herds on an island with a BHV1 control programme. Epidemiol. Infect. 130, 541-552. 
Heffernan, J.M., Smith, R.J., Wahl, L.M., 2005. Perspectives on the basic reproductive ratio. J. R. Soc. Interface 2, 281-293.

Kroese, A.H., De Jong, M.C.M., 2001. Design and analysis of transmission experiments. In: Menzies, F.D., Reid, S.W.J. (Ed.), Annual Meeting of the Society for Veterinary Epidemiology and Preventive Medicine, Noordwijkerhout, The Netherlands, xxi-xxxvii.

Lubinga, J.C., Tuppurainen, E.S., Coetzer, J.A., Stoltsz, W.H., Venter, E.H., 2014.

Evidence of lumpy skin disease virus over-wintering by transstadial persistence in Amblyomma hebraeum and transovarial persistence in Rhipicephalus decoloratus ticks. Exp. Appl. Acarolo. 62, 77-90.

Lubinga, J.C., Tuppurainen, E.S., Stoltsz, W.H., Ebersohn, K., Coetzer, J.A., Venter, E.H., 2013. Detection of lumpy skin disease virus in saliva of ticks fed on lumpy skin disease virus-infected cattle. Exp. Appl. Acarol. 61, 129-138.

Magori-Cohen, R., Louzoun, Y., Herziger, Y., Oron, E., Arazi, A., Tuppurainen, E., Shpigel, N.Y., Klement, E., 2012. Mathematical modelling and evaluation of the different routes of transmission of lumpy skin disease virus. Vet. Res. 43. doi: 10.1186/1297-9716-43-1.

NMA, 2013. Annual climate bulletin., National meteorological agency (NMA), Addis ababa Ethiopia.

http://www.ethiomet.gov.et/bulletins/view_pdf/348/2013_annual_bull etin.pdf. Accessed 30 June, 2016.

O’Neill, P.D., 2010. Introduction and snapshot review: Relating infectious disease transmission models to data. Stat. Med. 29, 2069-2077.

OIE, 2010. Manual of diagnostic tests and vaccines for terrestrial animals, chapter 2.4.14, Lumpy skin disease. OIE, Paris. http://web.oie.int/eng/normes/MMANUAL/A_Index.htm. Accessed 26 February 2016. OIE, Paris.

OIE, 2013. Lumpy skin disease. OIE Technical Disease Cards. OIE.

Stegeman, A., Elbers, A.R.W., Bouma, A., De Smit, H., De Jong, M.C.M., 1999.

Transmission of classical swine fever virus within herds during the 19971998 epidemic in The Netherlands. Prev. Vet. Med. 42, 201-218.

Tuppurainen, E.S., Lubinga, J.C., Stoltsz, W.H., Troskie, M., Carpenter, S.T., Coetzer, J.A., Venter, E.H., Oura, C.A., 2013a. Evidence of vertical transmission of lumpy skin disease virus in Rhipicephalus decoloratus ticks. Ticks Tickborne Dis. 4, 329-333.

Tuppurainen, E.S., Lubinga, J.C., Stoltsz, W.H., Troskie, M., Carpenter, S.T., Coetzer, J.A., Venter, E.H., Oura, C.A., 2013b. Mechanical transmission of lumpy skin 
disease virus by Rhipicephalus appendiculatus male ticks. Epidemiol. Infect. 141, 425-430.

Tuppurainen, E.S., Oura, C.A., 2012. Review: lumpy skin disease: an emerging threat to Europe, the Middle East and Asia. Transbound. Emerg. Dis. 59, 40-48.

Tuppurainen, E.S., Stoltsz, W.H., Troskie, M., Wallace, D.B., Oura, C.A., Mellor, P.S., Coetzer, J.A., Venter, E.H., 2011. A potential role for ixodid (hard) tick vectors in the transmission of lumpy skin disease virus in cattle.

Transbound. Emerg. Dis. 58, 93-104.

Tuppurainen, E.S., Venter, E.H., Shisler, J.L., Gari, G., Mekonnen, G.A., Juleff, N., Lyons, N.A., De Clercq, K., Upton, C., Bowden, T.R., Babiuk, S., Babiuk, L.A., 2015. Review: Capripoxvirus Diseases: Current Status and Opportunities for Control. Transbound. Emerg. Dis. Published online: 29 July 2015. doi:101111/tbed12444.

Tuppurainen, E.S.M., Venter, E.H., Coetzer, J.A.W., 2005. The detection of lumpy skin disease virus in samples of experimentally infected cattle using different diagnostic techniques. Onderstepoort J. Vet. Res. 72, 153-164.

van Roermund, H.J.W., Eblé, P.L., De Jong, M.C.M., Dekker, A., 2010 No betweenpen transmission of foot-and-mouth disease virus in vaccinated pigs. Vaccine 28, 4452-4461.

Velthuis, A.G.J., De Jong, M.C.M., De Bree, J., G., N., van Boven, M., 2002. Quantification of transmission in one-to-one experiments. Epidemiol. Infect. 128, 193-204.

Velthuis, A.G.J., De Jong, M.C.M., Kamp, E.M., Stockhofe, N., Verheijden, J.H.M., 2003. Design and analysis of an Actinobacillus pleuropneumoniae transmission experiment. Prev. Vet. Med. 60, 53-68.

Woods, J.A., 1988. Lumpy skin disease- A review. Trop. Anim. Hlth. Prod. 20, 11-17.

Woods, J.A., 1990. Lumpy skin disease virus. In: Dinter, Z., Morein, B. eds. Virus infections of ruminants. Elsevier Science publishers B. V. Amesterdam. pp. 53-67. 


\section{Appendixes}

Appendix 4.1. Number of newly infected (C), infectious (I), susceptible (S) animals, contribution of the environment to the transmission (E) and total number of animals $(\mathrm{N})$ of the epidemic during the 2014 LSD outbreak in Mota area.

\begin{tabular}{|c|c|c|c|c|c|c|}
\hline Period & $\begin{array}{l}\text { Date of } \\
\text { infection }\end{array}$ & $S$ & 1 & $\mathrm{E}$ & $\mathrm{C}$ & $\mathrm{N}$ \\
\hline 1 & 29-4-2014 & 12508 & 1 & 0 & 0 & 12509 \\
\hline 1 & $30-4-2014$ & 12508 & 1 & 0.325 & 0 & 12509 \\
\hline 1 & $1-5-2014$ & 12508 & 1 & 0.430625 & 0 & 12509 \\
\hline 1 & 2-5-2014 & 12508 & 1 & 0.464953125 & 0 & 12509 \\
\hline 1 & 3-5-2014 & 12508 & 1 & 0.476109766 & 0 & 12509 \\
\hline 1 & $4-5-2014$ & 12508 & 1 & 0.479735674 & 1 & 12509 \\
\hline 1 & 5-5-2014 & 12507 & 2 & 0.480914094 & 0 & 12509 \\
\hline 1 & $6-5-2014$ & 12507 & 2 & 0.806297081 & 0 & 12509 \\
\hline 1 & $7-5-2014$ & 12507 & 2 & 0.912046551 & 0 & 12509 \\
\hline 1 & 8-5-2014 & 12507 & 2 & 0.946415129 & 0 & 12509 \\
\hline 1 & $9-5-2014$ & 12507 & 1 & 0.957584917 & 0 & 12509 \\
\hline 1 & $10-5-2014$ & 12507 & 1 & 0.636215098 & 1 & 12509 \\
\hline 1 & $11-5-2014$ & 12506 & 2 & 0.531769907 & 0 & 12509 \\
\hline 1 & $12-5-2014$ & 12506 & 2 & 0.82282522 & 1 & 12509 \\
\hline 1 & $13-5-2014$ & 12505 & 3 & 0.917418196 & 0 & 12509 \\
\hline 1 & $14-5-2014$ & 12505 & 3 & 1.273160914 & 0 & 12509 \\
\hline 1 & $15-5-2014$ & 12505 & 2 & 1.388777297 & 1 & 12509 \\
\hline 1 & $16-5-2014$ & 12504 & 3 & 1.101352622 & 0 & 12509 \\
\hline 1 & $17-5-2014$ & 12504 & 3 & 1.332939602 & 0 & 12509 \\
\hline 1 & $18-5-2014$ & 12504 & 3 & 1.408205371 & 0 & 12509 \\
\hline 1 & $19-5-2014$ & 12504 & 3 & 1.432666745 & 1 & 12508 \\
\hline 1 & $20-5-2014$ & 12503 & 4 & 1.440616692 & 0 & 12508 \\
\hline
\end{tabular}




\begin{tabular}{|c|c|c|c|c|c|c|}
\hline 1 & $21-5-2014$ & 12503 & 3 & 1.768200425 & 1 & 12508 \\
\hline 1 & $22-5-2014$ & 12502 & 4 & 1.549665138 & 0 & 12508 \\
\hline 1 & $23-5-2014$ & 12502 & 3 & 1.80364117 & 1 & 12507 \\
\hline 1 & $24-5-2014$ & 12501 & 4 & 1.56118338 & 1 & 12507 \\
\hline 1 & $25-5-2014$ & 12500 & 5 & 1.807384599 & 1 & 12507 \\
\hline 1 & $26-5-2014$ & 12499 & 5 & 2.212399995 & 0 & 12507 \\
\hline 1 & $27-5-2014$ & 12499 & 5 & 2.344029998 & 0 & 12507 \\
\hline 1 & $28-5-2014$ & 12499 & 5 & 2.386809749 & 0 & 12507 \\
\hline 1 & $29-5-2014$ & 12499 & 5 & 2.400713169 & 1 & 12507 \\
\hline 1 & $30-5-2014$ & 12498 & 5 & 2.40523178 & 1 & 12507 \\
\hline 1 & $31-5-2014$ & 12497 & 6 & 2.406700328 & 0 & 12507 \\
\hline 1 & $1-6-2014$ & 12497 & 5 & 2.732177607 & 0 & 12507 \\
\hline 2 & 2-6-2014 & 12497 & 5 & 2.512957722 & 0 & 12507 \\
\hline 2 & $3-6-2014$ & 12497 & 4 & 2.44171126 & 0 & 12507 \\
\hline 2 & $4-6-2014$ & 12497 & 3 & 2.093556159 & 1 & 12507 \\
\hline 2 & $5-6-2014$ & 12496 & 3 & 1.655405752 & 0 & 12507 \\
\hline 2 & 6-6-2014 & 12496 & 3 & 1.513006869 & 1 & 12507 \\
\hline 2 & 7-6-2014 & 12495 & 4 & 1.466727233 & 0 & 12507 \\
\hline 2 & 8-6-2014 & 12495 & 4 & 1.776686351 & 0 & 12507 \\
\hline 2 & 9-6-2014 & 12495 & 3 & 1.877423064 & 0 & 12507 \\
\hline 2 & $10-6-2014$ & 12495 & 2 & 1.585162496 & 0 & 12507 \\
\hline 2 & $11-6-2014$ & 12495 & 2 & 1.165177811 & 1 & 12507 \\
\hline 2 & $12-6-2014$ & 12494 & 3 & 1.028682789 & 1 & 12507 \\
\hline 2 & $13-6-2014$ & 12493 & 4 & 1.309321906 & 1 & 12507 \\
\hline 2 & $14-6-2014$ & 12492 & 5 & 1.72552962 & 1 & 12506 \\
\hline 2 & $15-6-2014$ & 12491 & 5 & 2.185797126 & 0 & 12506 \\
\hline 2 & $16-6-2014$ & 12491 & 5 & 2.335384066 & 1 & 12506 \\
\hline 2 & $17-6-2014$ & 12490 & 5 & 2.383999821 & 2 & 12506 \\
\hline 2 & $18-6-2014$ & 12488 & 7 & 2.399799942 & 2 & 12506 \\
\hline 2 & $19-6-2014$ & 12486 & 9 & 3.054934981 & 0 & 12506 \\
\hline 2 & $20-6-2014$ & 12486 & 9 & 3.917853869 & 0 & 12506 \\
\hline 2 & $21-6-2014$ & 12486 & 9 & 4.198302507 & 2 & 12506 \\
\hline
\end{tabular}




\begin{tabular}{|c|c|c|c|c|c|c|}
\hline 2 & 22-6-2014 & 12484 & 10 & 4.289448315 & 3 & 12506 \\
\hline 2 & 23-6-2014 & 12481 & 12 & 4.644070702 & 0 & 12505 \\
\hline 2 & 24-6-2014 & 12481 & 11 & 5.409322978 & 0 & 12505 \\
\hline 2 & $25-6-2014$ & 12481 & 10 & 5.333029968 & 4 & 12505 \\
\hline 2 & $26-6-2014$ & 12477 & 13 & 4.98323474 & 0 & 12505 \\
\hline 2 & 27-6-2014 & 12477 & 12 & 5.84455129 & 5 & 12505 \\
\hline 2 & $28-6-2014$ & 12472 & 15 & 5.799479169 & 1 & 12505 \\
\hline 2 & $29-6-2014$ & 12471 & 14 & 6.75983073 & 0 & 12505 \\
\hline 2 & $30-6-2014$ & 12471 & 14 & 6.746944987 & 2 & 12505 \\
\hline 2 & $1-7-2014$ & 12469 & 16 & 6.742757121 & 1 & 12505 \\
\hline 2 & $2-7-2014$ & 12468 & 15 & 7.391396064 & 0 & 12505 \\
\hline 2 & $3-7-2014$ & 12468 & 13 & 7.277203721 & 6 & 12505 \\
\hline 2 & 4-7-2014 & 12462 & 19 & 6.590091209 & 6 & 12505 \\
\hline 2 & $5-7-2014$ & 12456 & 25 & 8.316779643 & 3 & 12505 \\
\hline 2 & 6-7-2014 & 12453 & 24 & 10.82795338 & 5 & 12505 \\
\hline 2 & $7-7-2014$ & 12448 & 29 & 11.31908485 & 3 & 12504 \\
\hline 2 & 8-7-2014 & 12445 & 27 & 13.10370258 & 8 & 12504 \\
\hline 2 & $9-7-2014$ & 12437 & 34 & 13.03370334 & 6 & 12504 \\
\hline 2 & $10-7-2014$ & 12431 & 40 & 15.28595358 & 4 & 12504 \\
\hline 2 & $11-7-2014$ & 12427 & 42 & 17.96793491 & 8 & 12504 \\
\hline 2 & $12-7-2014$ & 12419 & 49 & 19.48957885 & 3 & 12504 \\
\hline 2 & $13-7-2014$ & 12416 & 52 & 22.25911313 & 2 & 12503 \\
\hline 2 & $14-7-2014$ & 12414 & 48 & 24.13421177 & 6 & 12503 \\
\hline 2 & $15-7-2014$ & 12408 & 48 & 23.44361882 & 4 & 12503 \\
\hline 2 & $16-7-2014$ & 12404 & 49 & 23.21917612 & 5 & 12503 \\
\hline 2 & $17-7-2014$ & 12399 & 49 & 23.47123224 & 1 & 12502 \\
\hline 2 & $18-7-2014$ & 12398 & 47 & 23.55315048 & 14 & 12502 \\
\hline 2 & 19-7-2014 & 12384 & 53 & 22.92977391 & 9 & 12502 \\
\hline 2 & 20-7-2014 & 12375 & 56 & 24.67717652 & 3 & 12502 \\
\hline 2 & 21-7-2014 & 12372 & 55 & 26.22008237 & 5 & 12502 \\
\hline 2 & $22-7-2014$ & 12367 & 52 & 26.39652677 & 12 & 12501 \\
\hline 2 & 23-7-2014 & 12355 & 61 & 25.4788712 & 8 & 12501 \\
\hline 2 & $24-7-2014$ & 12347 & 67 & 28.10563314 & 11 & 12501 \\
\hline 2 & $25-7-2014$ & 12336 & 72 & 30.90933077 & 5 & 12500 \\
\hline
\end{tabular}




\begin{tabular}{|c|c|c|c|c|c|c|}
\hline 2 & $26-7-2014$ & 12331 & 73 & 33.4455325 & 11 & 12500 \\
\hline 2 & 27-7-2014 & 12320 & 78 & 34.59479806 & 12 & 12499 \\
\hline 2 & 28-7-2014 & 12308 & 89 & 36.59330937 & 6 & 12498 \\
\hline 2 & 29-7-2014 & 12302 & 82 & 40.81782555 & 4 & 12498 \\
\hline 2 & $30-7-2014$ & 12298 & 77 & 39.9157933 & 6 & 12497 \\
\hline 2 & $31-7-2014$ & 12292 & 79 & 37.99763282 & 6 & 12497 \\
\hline 2 & $1-8-2014$ & 12286 & 80 & 38.02423067 & 4 & 12497 \\
\hline 2 & 2-8-2014 & 12282 & 72 & 38.35787497 & 10 & 12496 \\
\hline 2 & $3-8-2014$ & 12272 & 74 & 35.86630936 & 7 & 12495 \\
\hline 2 & 4-8-2014 & 12265 & 70 & 35.70655054 & 6 & 12495 \\
\hline 2 & $5-8-2014$ & 12259 & 71 & 34.35462893 & 9 & 12495 \\
\hline 2 & $6-8-2014$ & 12250 & 69 & 34.2402544 & 10 & 12494 \\
\hline 2 & 7-8-2014 & 12240 & 68 & 33.55308268 & 12 & 12492 \\
\hline 2 & 8-8-2014 & 12228 & 74 & 33.00475187 & 12 & 12492 \\
\hline 2 & 9-8-2014 & 12216 & 82 & 34.77654436 & 6 & 12492 \\
\hline 2 & $10-8-2014$ & 12210 & 82 & 37.95237692 & 15 & 12492 \\
\hline 2 & $11-8-2014$ & 12195 & 91 & 38.9845225 & 6 & 12491 \\
\hline 2 & $12-8-2014$ & 12189 & 93 & 42.24496981 & 10 & 12490 \\
\hline 2 & $13-8-2014$ & 12179 & 92 & 43.95461519 & 10 & 12489 \\
\hline 2 & $14-8-2014$ & 12169 & 96 & 44.18524994 & 7 & 12489 \\
\hline 2 & $15-8-2014$ & 12162 & 96 & 45.56020623 & 16 & 12488 \\
\hline 2 & $16-8-2014$ & 12146 & 103 & 46.00706702 & 14 & 12487 \\
\hline 2 & $17-8-2014$ & 12132 & 107 & 48.42729678 & 10 & 12487 \\
\hline 2 & $18-8-2014$ & 12122 & 104 & 50.51387145 & 4 & 12484 \\
\hline 2 & $19-8-2014$ & 12118 & 96 & 50.21700822 & 6 & 12484 \\
\hline 2 & $20-8-2014$ & 12112 & 95 & 47.52052767 & 15 & 12483 \\
\hline 2 & 21-8-2014 & 12097 & 95 & 46.31917149 & 14 & 12482 \\
\hline 2 & $22-8-2014$ & 12083 & 101 & 45.92873074 & 22 & 12479 \\
\hline 2 & 23-8-2014 & 12061 & 115 & 47.75183749 & 13 & 12478 \\
\hline 2 & $24-8-2014$ & 12048 & 118 & 52.89434718 & 5 & 12478 \\
\hline 2 & $25-8-2014$ & 12043 & 117 & 55.54066283 & 6 & 12476 \\
\hline 2 & $26-8-2014$ & 12037 & 107 & 56.07571542 & 14 & 12474 \\
\hline
\end{tabular}




\begin{tabular}{|c|c|c|c|c|c|c|}
\hline 2 & $27-8-2014$ & 12023 & 108 & 52.99960751 & 8 & 12473 \\
\hline 2 & 28-8-2014 & 12015 & 106 & 52.32487244 & 14 & 12473 \\
\hline 2 & $29-8-2014$ & 12001 & 117 & 51.45558354 & 9 & 12472 \\
\hline 2 & $30-8-2014$ & 11992 & 120 & 54.74806465 & 5 & 12472 \\
\hline 2 & $31-8-2014$ & 11987 & 110 & 56.79312101 & 10 & 12472 \\
\hline 2 & $1-9-2014$ & 11977 & 106 & 54.20776433 & 13 & 12472 \\
\hline 2 & 2-9-2014 & 11964 & 97 & 52.06752341 & 9 & 12472 \\
\hline 2 & 3-9-2014 & 11955 & 93 & 48.44694511 & 12 & 12471 \\
\hline 2 & 4-9-2014 & 11943 & 100 & 45.97025716 & 9 & 12471 \\
\hline 2 & $5-9-2014$ & 11934 & 103 & 47.44033358 & 10 & 12471 \\
\hline 2 & $6-9-2014$ & 11924 & 99 & 48.89310841 & 6 & 12471 \\
\hline 2 & $7-9-2014$ & 11918 & 97 & 48.06526023 & 8 & 12471 \\
\hline 2 & 8-9-2014 & 11910 & 91 & 47.14620958 & 5 & 12469 \\
\hline 2 & $9-9-2014$ & 11905 & 87 & 44.89751811 & 9 & 12469 \\
\hline 2 & $10-9-2014$ & 11896 & 91 & 42.86669339 & 13 & 12469 \\
\hline 2 & $11-9-2014$ & 11883 & 94 & 43.50667535 & 13 & 12469 \\
\hline 2 & $12-9-2014$ & 11870 & 94 & 44.68966949 & 11 & 12469 \\
\hline 2 & $13-9-2014$ & 11859 & 96 & 45.07414258 & 5 & 12468 \\
\hline 2 & $14-9-2014$ & 11854 & 89 & 45.84909634 & 34 & 12468 \\
\hline 2 & $15-9-2014$ & 11820 & 113 & 43.82595631 & 7 & 12466 \\
\hline 2 & $16-9-2014$ & 11813 & 111 & 50.9684358 & 19 & 12465 \\
\hline 2 & $17-9-2014$ & 11794 & 123 & 52.63974164 & 15 & 12463 \\
\hline 2 & $18-9-2014$ & 11779 & 130 & 57.08291603 & 11 & 12462 \\
\hline 2 & $19-9-2014$ & 11768 & 136 & 60.80194771 & 16 & 12462 \\
\hline 2 & $20-9-2014$ & 11752 & 142 & 63.96063301 & 4 & 12461 \\
\hline 2 & $21-9-2014$ & 11748 & 133 & 66.93720573 & 28 & 12460 \\
\hline 2 & $22-9-2014$ & 11720 & 148 & 64.97959186 & 18 & 12458 \\
\hline 2 & $23-9-2014$ & 11702 & 155 & 69.21836735 & 9 & 12457 \\
\hline 2 & $24-9-2014$ & 11693 & 160 & 72.87096939 & 29 & 12456 \\
\hline 2 & $25-9-2014$ & 11664 & 154 & 75.68306505 & 28 & 12452 \\
\hline 2 & $26-9-2014$ & 11636 & 175 & 74.64699614 & 13 & 12451 \\
\hline 2 & 27-9-2014 & 11623 & 170 & 81.13527375 & 13 & 12450 \\
\hline 2 & $28-9-2014$ & 11610 & 169 & 81.61896397 & 19 & 12448 \\
\hline 3 & 29-9-2014 & 11591 & 177 & 81.45116329 & 12 & 12447 \\
\hline
\end{tabular}




\begin{tabular}{|c|c|c|c|c|c|c|}
\hline 3 & $30-9-2014$ & 11579 & 173 & 83.99662807 & 18 & 12446 \\
\hline 3 & $1-10-2014$ & 11561 & 187 & 83.52390412 & 12 & 12445 \\
\hline 3 & 2-10-2014 & 11549 & 170 & 87.92026884 & 12 & 12444 \\
\hline 3 & 3-10-2014 & 11537 & 162 & 83.82408737 & 14 & 12443 \\
\hline 3 & 4-10-2014 & 11523 & 166 & 79.8928284 & 9 & 12442 \\
\hline 3 & 5-10-2014 & 11514 & 148 & 79.91516923 & 13 & 12442 \\
\hline 3 & $6-10-2014$ & 11501 & 133 & 74.07243 & 18 & 12442 \\
\hline 3 & $7-10-2014$ & 11483 & 138 & 67.29853975 & 24 & 12442 \\
\hline 3 & 8-10-2014 & 11459 & 149 & 66.72202542 & 22 & 12441 \\
\hline 3 & 9-10-2014 & 11437 & 154 & 70.10965826 & 9 & 12439 \\
\hline 3 & $10-10-2014$ & 11428 & 151 & 72.83563893 & 6 & 12439 \\
\hline 3 & $11-10-2014$ & 11422 & 139 & 72.74658265 & 7 & 12439 \\
\hline 3 & $12-10-2014$ & 11415 & 134 & 68.81763936 & 9 & 12439 \\
\hline 3 & $13-10-2014$ & 11406 & 131 & 65.91573279 & 3 & 12439 \\
\hline 3 & $14-10-2014$ & 11403 & 120 & 63.99761316 & 15 & 12439 \\
\hline 3 & $15-10-2014$ & 11388 & 126 & 59.79922428 & 6 & 12438 \\
\hline 3 & $16-10-2014$ & 11382 & 119 & 60.38474789 & 5 & 12438 \\
\hline 3 & $17-10-2014$ & 11377 & 106 & 58.30004306 & 1 & 12438 \\
\hline 3 & $18-10-2014$ & 11376 & 83 & 53.397514 & 2 & 12438 \\
\hline 3 & 19-10-2014 & 11374 & 62 & 44.32919205 & 5 & 12437 \\
\hline 3 & 20-10-2014 & 11369 & 58 & 34.55698742 & 0 & 12437 \\
\hline 3 & 21-10-2014 & 11369 & 52 & 30.08102091 & 13 & 12437 \\
\hline 3 & 22-10-2014 & 11356 & 57 & 26.6763318 & 8 & 12435 \\
\hline 3 & $23-10-2014$ & 11348 & 56 & 27.19480783 & 4 & 12435 \\
\hline 3 & 24-10-2014 & 11344 & 57 & 27.03831255 & 8 & 12435 \\
\hline 3 & $25-10-2014$ & 11336 & 51 & 27.31245158 & 18 & 12435 \\
\hline 3 & $26-10-2014$ & 11318 & 63 & 25.45154676 & 2 & 12434 \\
\hline 3 & 27-10-2014 & 11316 & 59 & 28.7467527 & 3 & 12433 \\
\hline 3 & $28-10-2014$ & 11313 & 61 & 28.51769463 & 12 & 12432 \\
\hline 3 & $29-10-2014$ & 11301 & 71 & 29.09325075 & 8 & 12432 \\
\hline 3 & $30-10-2014$ & 11293 & 74 & 32.53030649 & 6 & 12432 \\
\hline 3 & $31-10-2014$ & 11287 & 80 & 34.62234961 & 9 & 12432 \\
\hline
\end{tabular}




\begin{tabular}{|r|r|r|r|r|r|r|}
\hline 3 & $1-11-2014$ & 11278 & 76 & 37.25226362 & 7 & 12432 \\
\hline 3 & $2-11-2014$ & 11271 & 76 & 36.80698568 & 3 & 12432 \\
\hline 3 & $3-11-2014$ & 11268 & 75 & 36.66227035 & 2 & 12432 \\
\hline 3 & $4-11-2014$ & 11266 & 70 & 36.29023786 & 4 & 12432 \\
\hline 3 & $5-11-2014$ & 11262 & 56 & 34.54432731 & 9 & 12432 \\
\hline 3 & $6-11-2014$ & 11253 & 63 & 29.42690637 & 5 & 12432 \\
\hline 3 & $7-11-2014$ & 11248 & 65 & 30.03874457 & 4 & 12432 \\
\hline 3 & $8-11-2014$ & 11244 & 57 & 30.88759199 & 0 & 12432 \\
\hline 3 & $9-11-2014$ & 11244 & 49 & 28.5634674 & 1 & 12432 \\
\hline 3 & $10-11-2014$ & 11243 & 44 & 25.2081269 & 0 & 12432 \\
\hline 3 & $11-11-2014$ & 11243 & 35 & 22.49264124 & 0 & 12432 \\
\hline 3 & $12-11-2014$ & 11243 & 28 & 18.6851084 & 0 & 12432 \\
\hline 3 & $13-11-2014$ & 11243 & 25 & 15.17266023 & 0 & 12432 \\
\hline 3 & $14-11-2014$ & 11243 & 23 & 13.05611458 & 0 & 12432 \\
\hline 3 & $15-11-2014$ & 11243 & 19 & 11.71823724 & 0 & 12432 \\
\hline 3 & $16-11-2014$ & 11243 & 10 & 9.983427102 & 0 & 12432 \\
\hline 3 & $17-11-2014$ & 11243 & 5 & 6.494613808 & 0 & 12432 \\
\hline 3 & $18-11-2014$ & 11243 & 1 & 3.735749488 & 0 & 12432 \\
\hline 3 & $19-11-2014$ & 11243 & 1 & 1.539118583 & 0 & 12432 \\
\hline
\end{tabular}



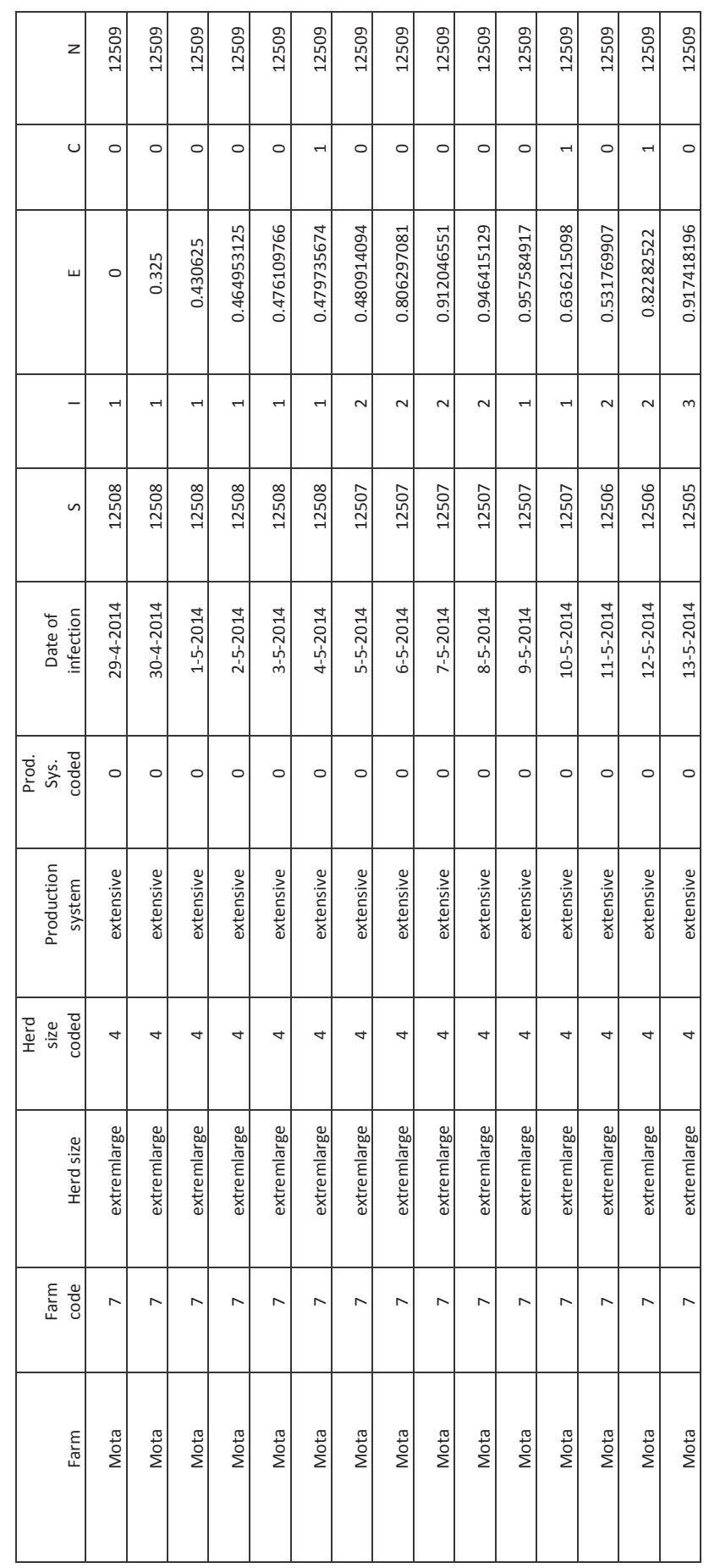


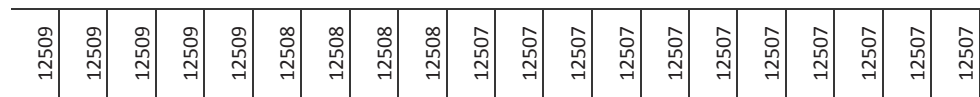

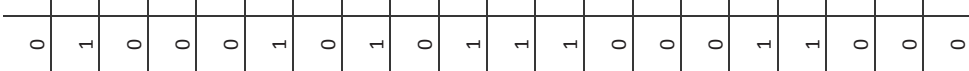

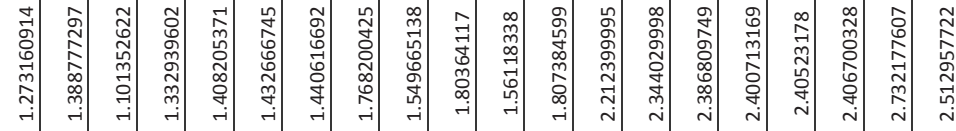

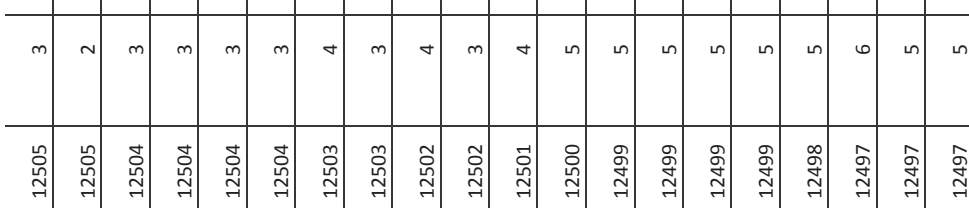

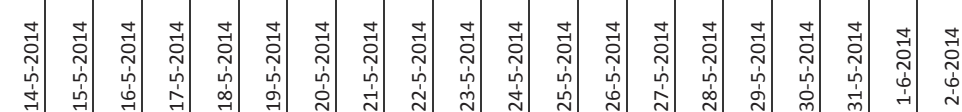

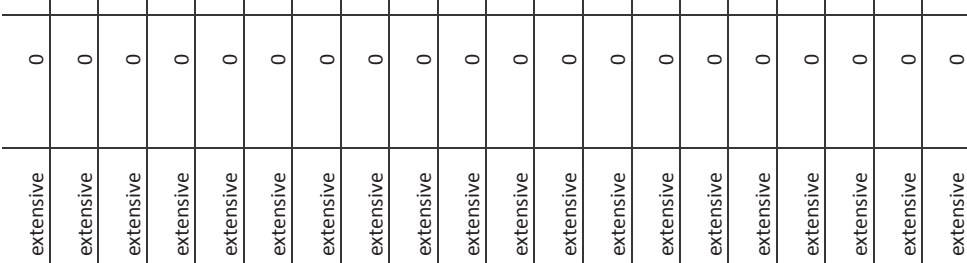
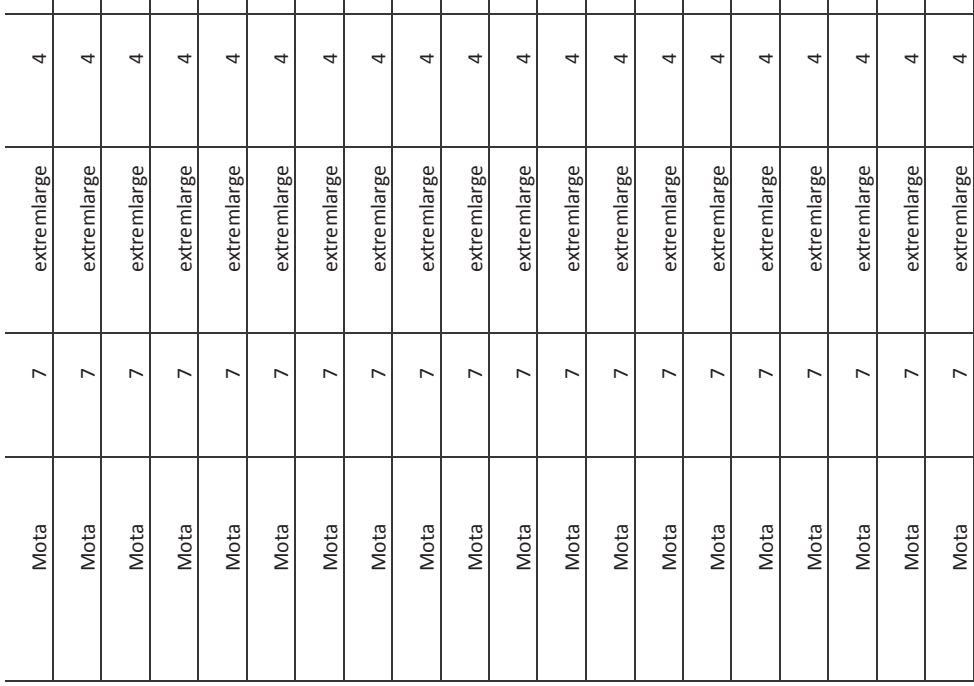

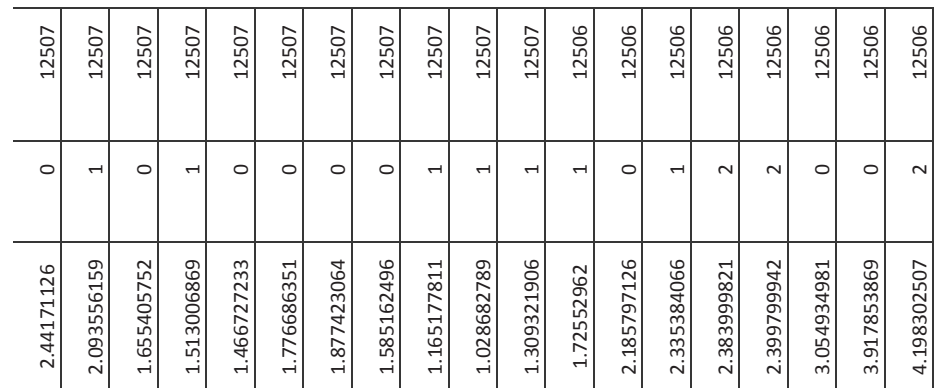

\begin{tabular}{|c|c|c|c|c|c|c|c|c|c|c|c|c|c|c|c|c|c|c|}
\hline$\nabla$ & $m$ & $m$ & $m$ & $\nabla$ & $ナ$ & $m$ & $N$ & $N$ & $m$ & 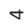 & เn & in & in & in & $N$ & $\sigma$ & $\sigma$ & $\sigma$ \\
\hline 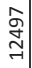 & 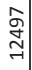 & $\underset{\stackrel{\mathscr{I}}{+}}{\stackrel{\sim}{\sim}}$ & $\begin{array}{l}\stackrel{\mathscr{g}}{+} \\
\stackrel{\sim}{\sim}\end{array}$ & $\underset{\stackrel{\sim}{\Omega}}{\stackrel{\sim}{\sim}}$ & 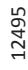 & $\underset{\stackrel{\text { I }}{+}}{\stackrel{\sim}{\sim}}$ & 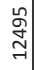 & 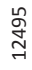 & $\underset{\stackrel{+}{+}}{\stackrel{+}{+}}$ & $\underset{\sim}{\stackrel{m}{\sim}}$ & $\underset{\text { ㄱ }}{\stackrel{\sim}{+}}$ & $\underset{\sim}{\stackrel{\sigma}{+}}$ & $\underset{\text { I্ }}{\stackrel{్}{+}}$ & $\underset{\text { 욤 }}{\stackrel{+}{+}}$ & $\begin{array}{l}\infty \\
\stackrel{0}{+} \\
\stackrel{\sim}{\sim}\end{array}$ & $\begin{array}{l}\text { } \\
\stackrel{\infty}{+} \\
\underset{\sim}{+}\end{array}$ & $\begin{array}{l}\infty \\
\stackrel{\infty}{+} \\
\underset{\sim}{\sim}\end{array}$ & $\begin{array}{l}\stackrel{0}{ } \\
\stackrel{\infty}{+} \\
\stackrel{\sim}{\sim}\end{array}$ \\
\hline
\end{tabular}

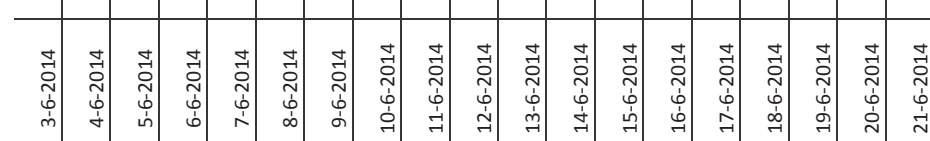
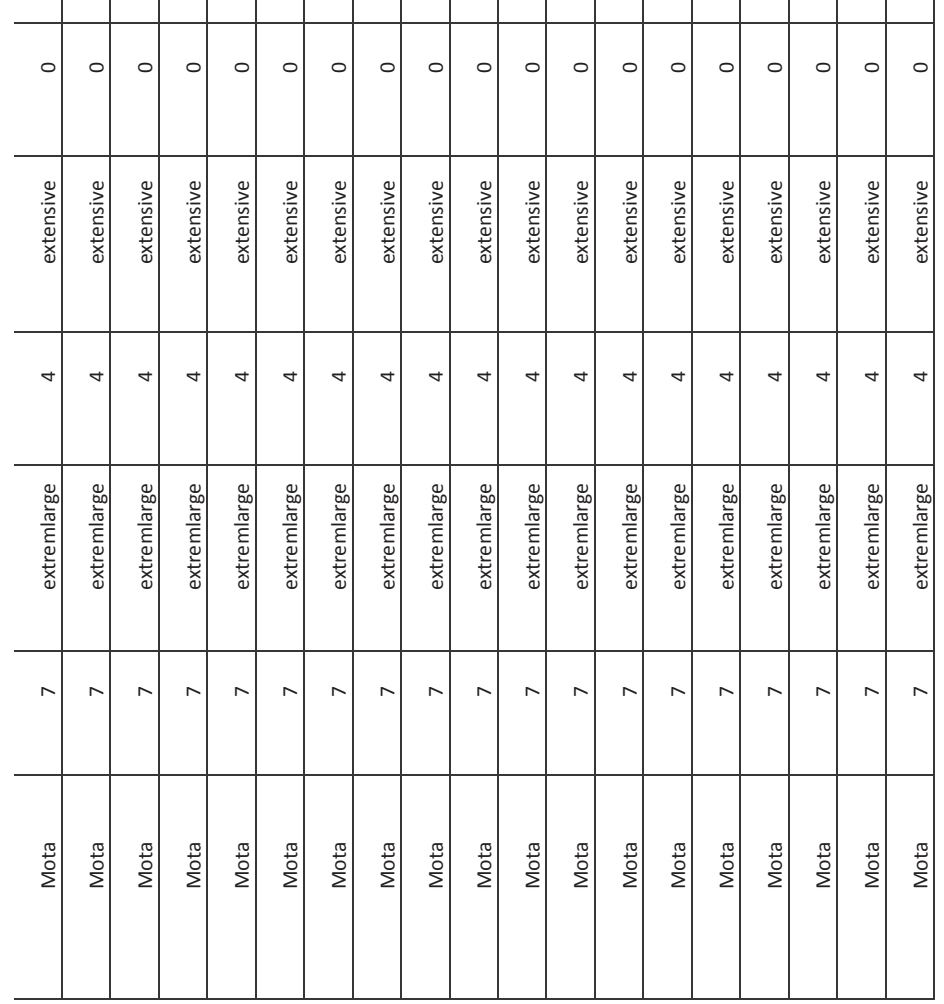


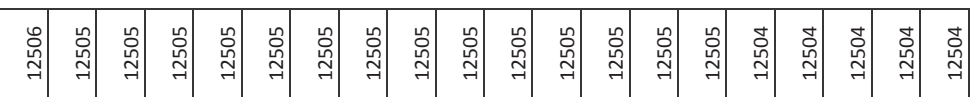

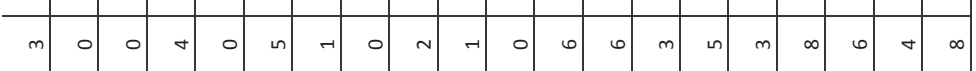

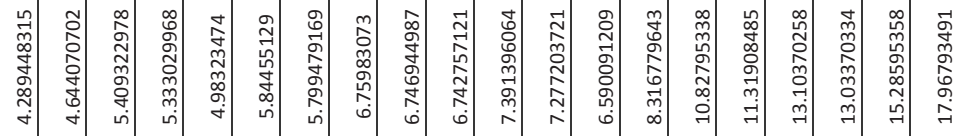

이 기

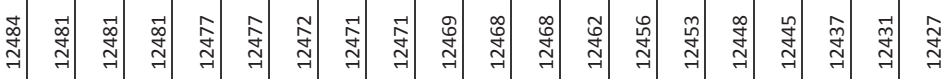

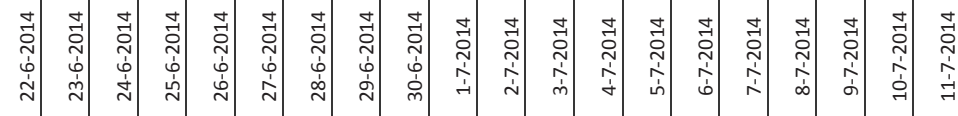

\begin{tabular}{llllllllllllllllllllll}
\hline & 0 & 0 & 0 & 0 & 0 & 0 & 0 & 0 & 0 & 0 & 0 & 0 & 0 & 0 & 0 & 0 & 0 & 0 & 0
\end{tabular}

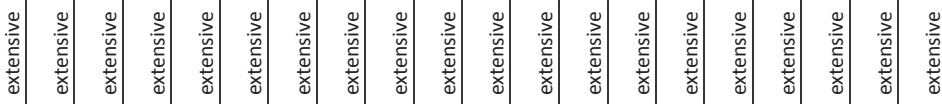

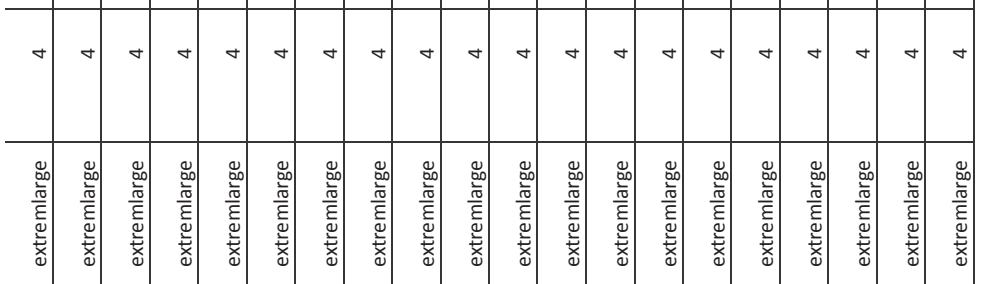

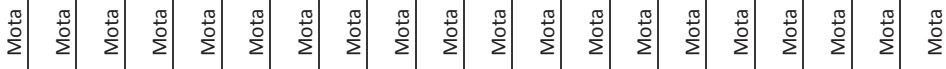




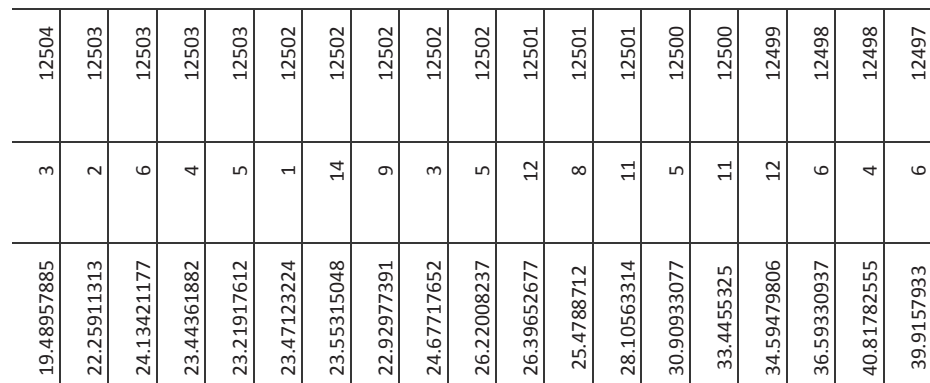

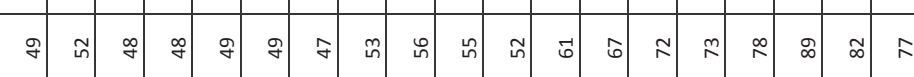

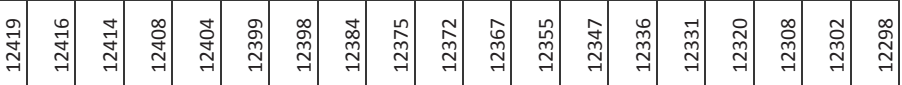
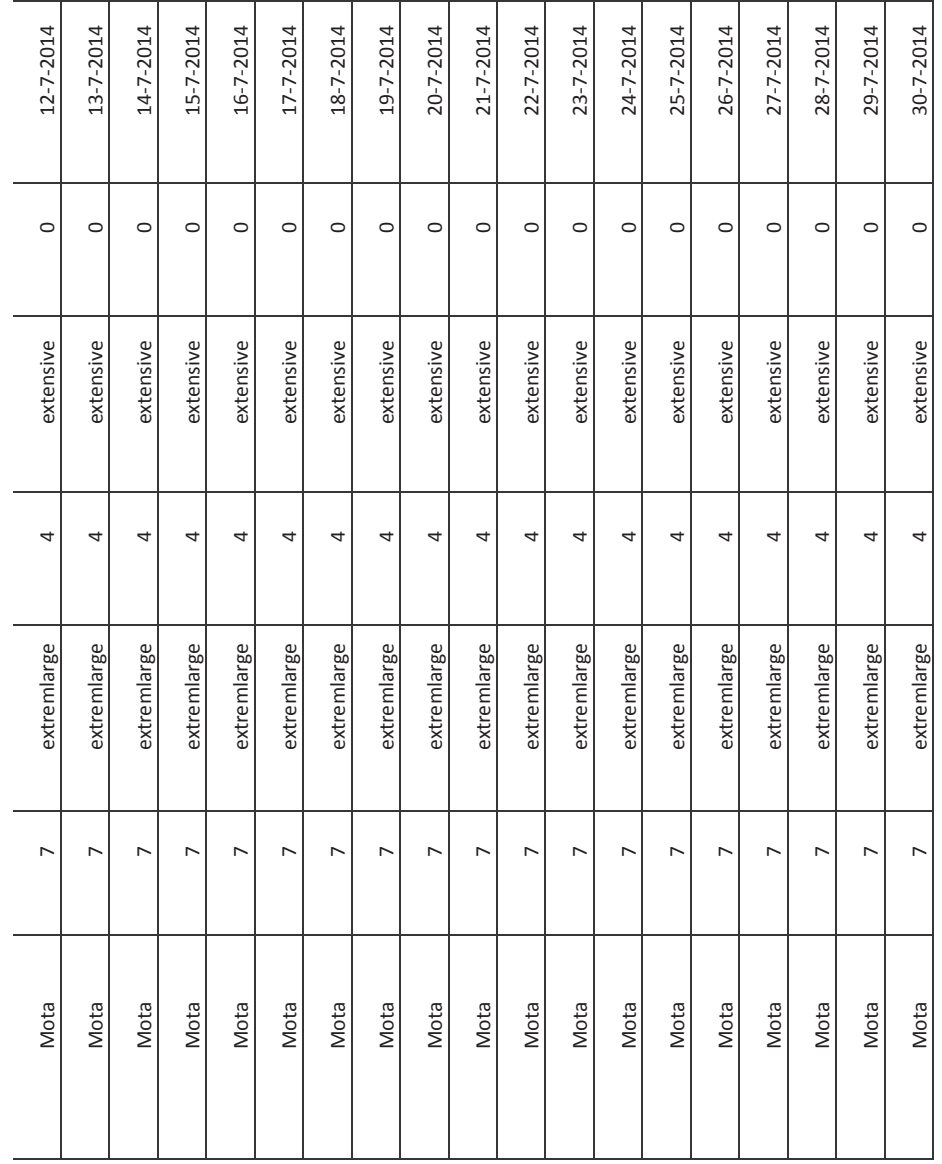


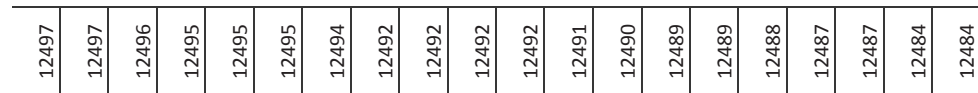

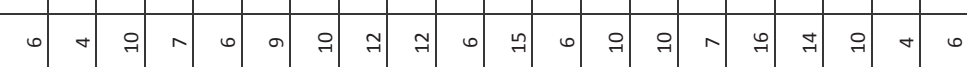

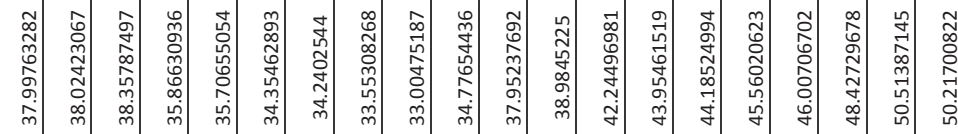

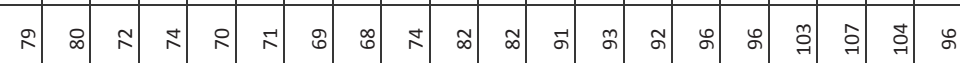

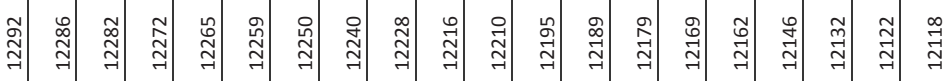

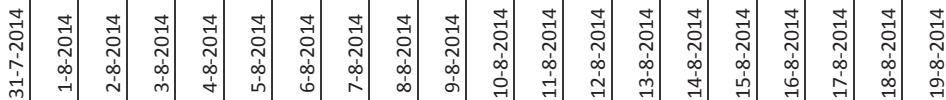
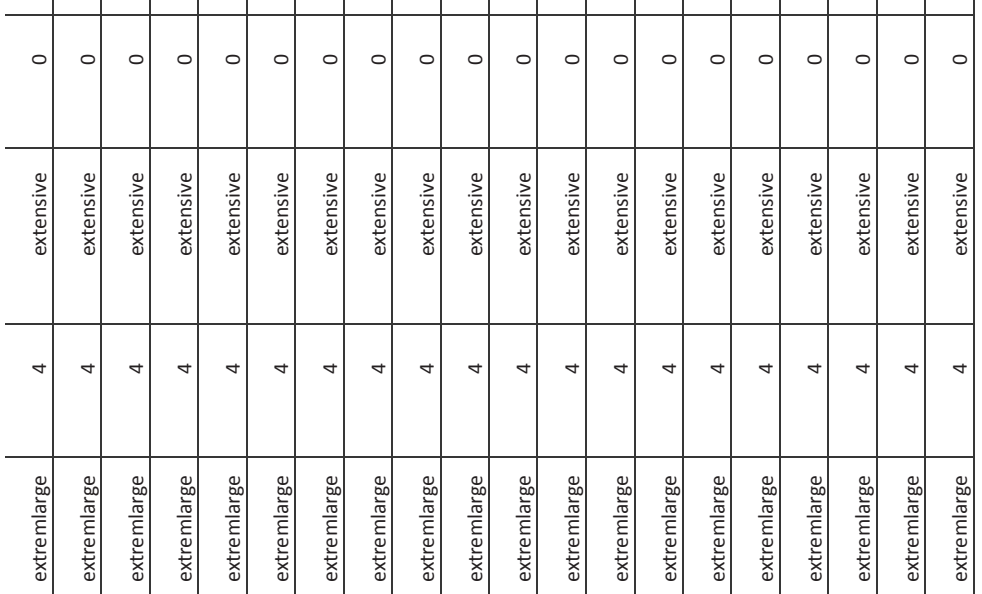

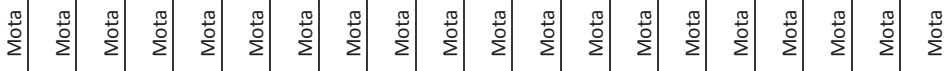




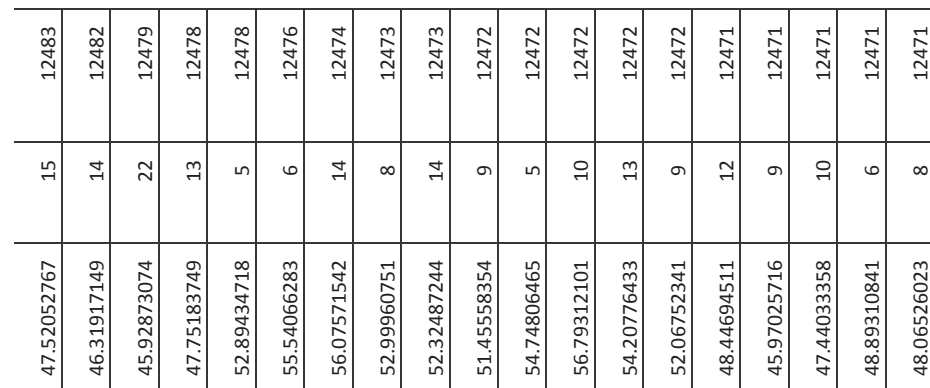

ถู

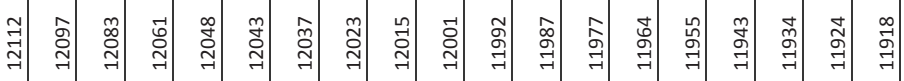

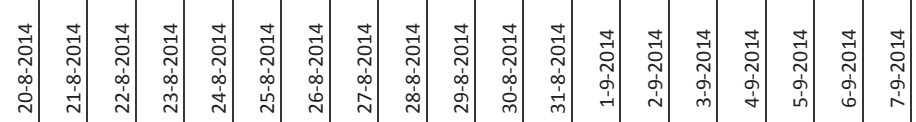

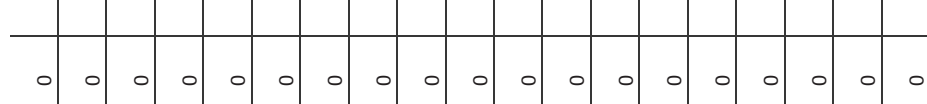

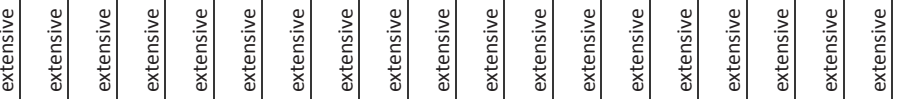

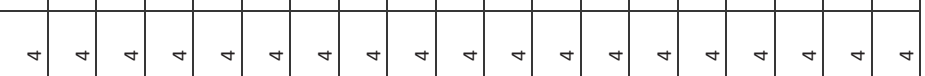

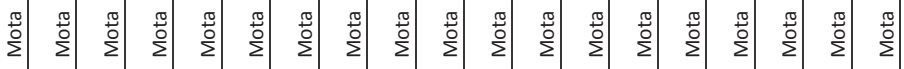




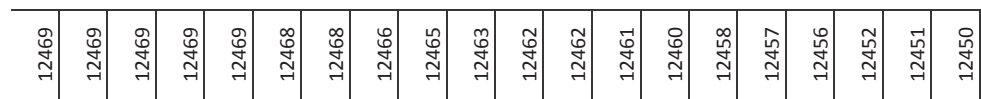

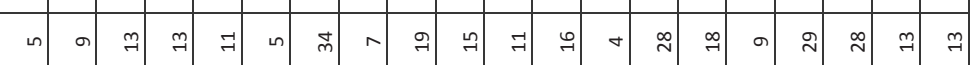

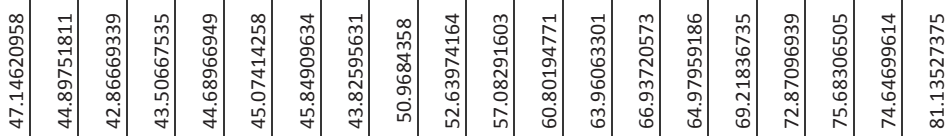

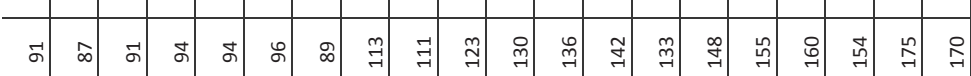

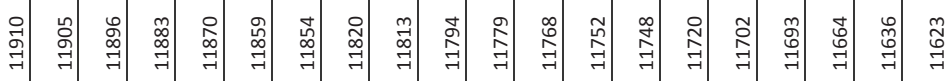

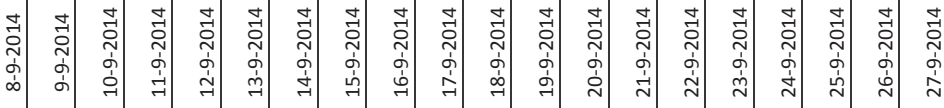

\begin{tabular}{llllll|l|l|l|l|l|l|l|l|l|l|l|l|l|l}
\hline & 0 & 0 & 0 & 0 & 0 & 0 & 0 & 0 & 0 & 0 & 0 & 0 & 0 & 0 & 0 & 0 & 0 & 0 & 0
\end{tabular}

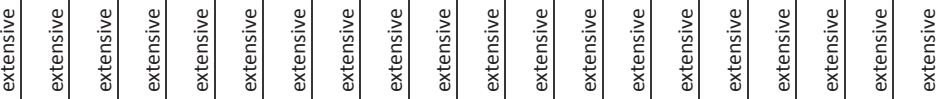

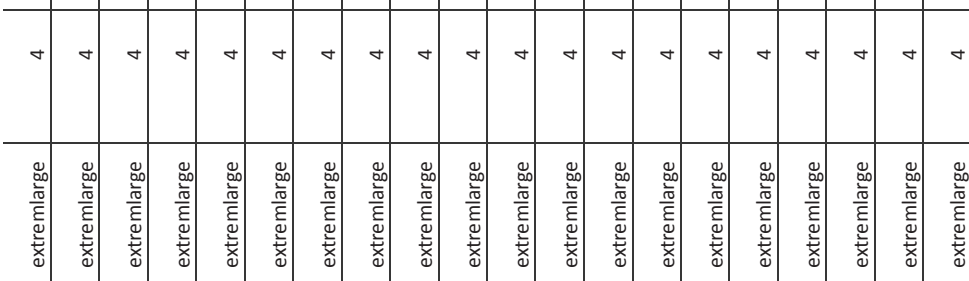

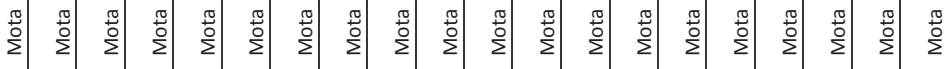




\begin{tabular}{|c|c|c|c|c|c|c|c|c|c|c|c|c|c|c|c|c|c|c|}
\hline 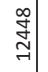 & 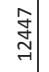 & \begin{tabular}{l}
0 \\
\multirow{J}{*}{} \\
\multirow{J}{*}{}
\end{tabular} & 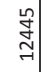 & $\begin{array}{l}\mathbb{J} \\
\underset{\exists}{J}\end{array}$ & 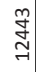 & $\underset{\mathbb{J}}{\mathbb{Z}}$ & 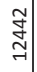 & 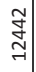 & $\begin{array}{l}\mathcal{J} \\
\underset{7}{*}\end{array}$ & $\begin{array}{l}\vec{J} \\
\underset{7}{7}\end{array}$ & 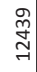 & 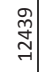 & $\begin{array}{l}\stackrel{q}{\tilde{g}} \\
\underset{\sim}{\mid}\end{array}$ & 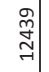 & $\begin{array}{l}\vec{q} \\
\underset{\sim}{\sim}\end{array}$ & 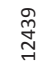 & & \\
\hline ᄀ & $\approx$ & $\stackrel{\infty}{\sim}$ & $\approx$ & $\approx$ & $\underset{A}{ }$ & $\sigma$ & $m$ & $\stackrel{\infty}{\sim}$ & $\stackrel{\sim}{\sim}$ & $\approx$ & $\sigma$ & 0 & $r$ & $\sigma$ & $m$ & 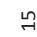 & 0 & \\
\hline $\begin{array}{l}0 \\
0 \\
0 \\
0 \\
0 \\
0 \\
-1 \\
\infty\end{array}$ & 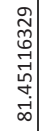 & 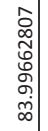 & 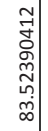 & 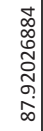 & 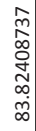 & 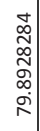 & 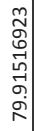 & $\begin{array}{l}\stackrel{M}{\mathbf{D}} \\
\mathbf{0} \\
\dot{\sim}\end{array}$ & 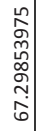 & 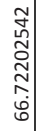 & $\begin{array}{l}\stackrel{0}{ } \\
0 \\
0 \\
0 \\
0 \\
0 \\
\stackrel{2}{n}\end{array}$ & $\begin{array}{l}0 \\
0 \\
0 \\
0 \\
0 \\
\infty \\
\stackrel{N}{N} \\
\end{array}$ & 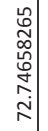 & $\begin{array}{l}0 \\
0 \\
0 \\
0 \\
0 \\
0 \\
0 \\
0 \\
0\end{array}$ & $\begin{array}{l}\hat{g} \\
\hat{n} \\
\hat{n} \\
\hat{\sigma} \\
\dot{0}\end{array}$ & 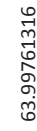 & & \\
\hline
\end{tabular}

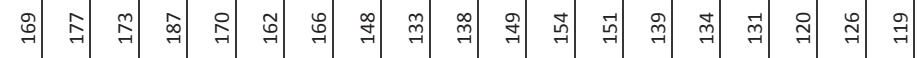

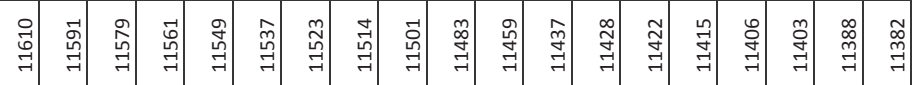

\begin{tabular}{|c|c|c|c|c|c|c|c|c|c|c|c|c|c|c|c|c|c|c|c|c|}
\hline 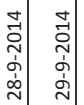 & $\begin{array}{l}0 \\
0 \\
0 \\
0 \\
0 \\
0\end{array}$ & 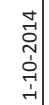 & 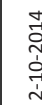 & & & 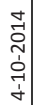 & $\begin{array}{l}\overrightarrow{0} \\
0 \\
\vdots \\
\text { ஸे }\end{array}$ & $\bar{c}$ & & & & & & & & & & & $\begin{array}{l}\overrightarrow{0} \\
\vdots \\
0 \\
\dot{d} \\
\dot{d}\end{array}$ & 乌் \\
\hline
\end{tabular}

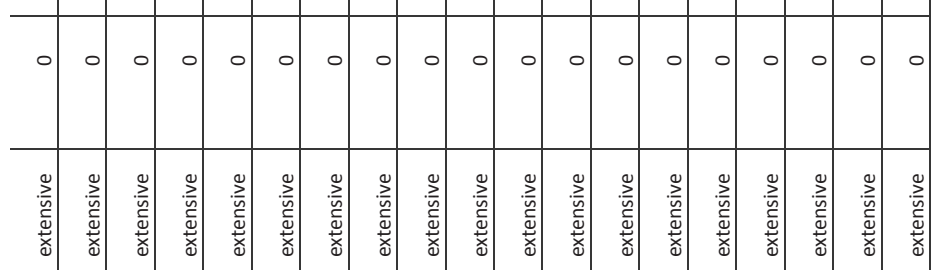
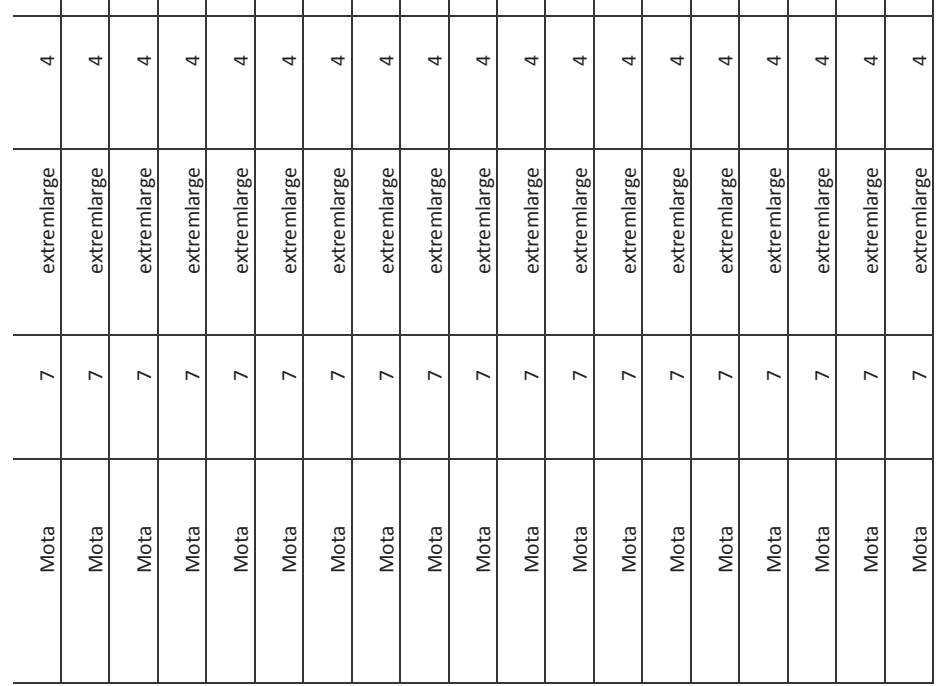


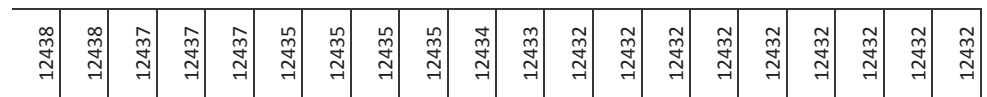

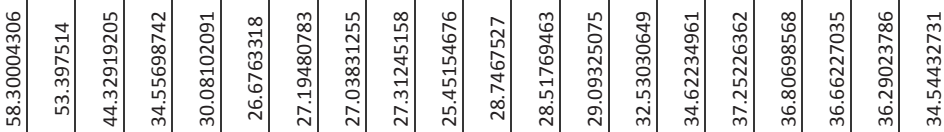

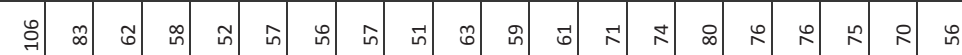

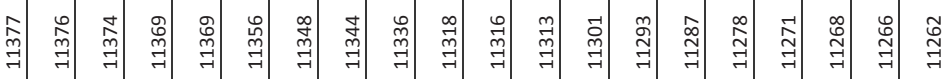

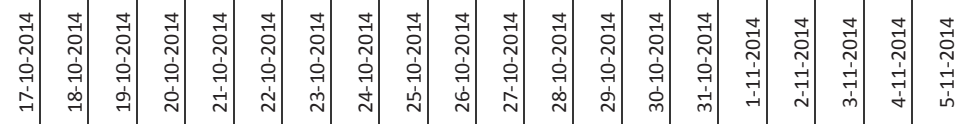

\begin{tabular}{llllllllllll|l|l|l|l|l|l|l|l|l}
\hline & 0 & 0 & 0 & 0 & 0 & 0 & 0 & 0 & 0 & 0 & 0 & 0 & 0 & 0 & 0 & 0 & 0 & 0 & 0
\end{tabular}

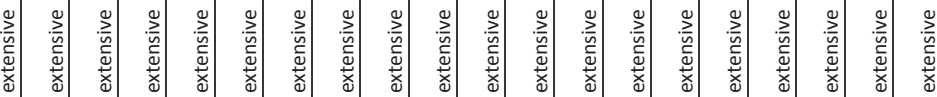

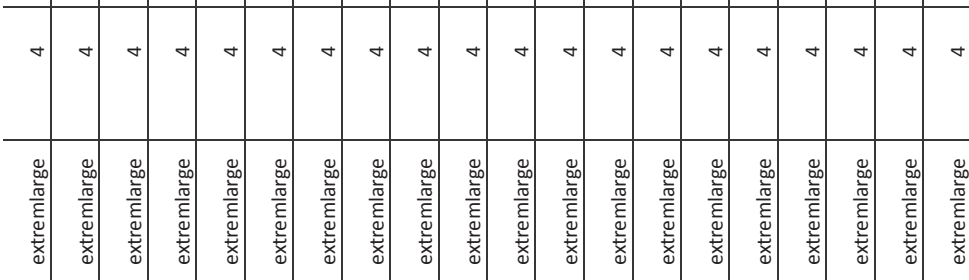

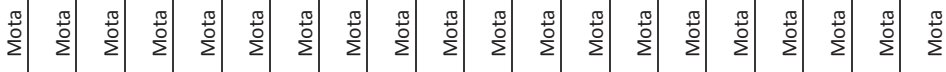




\begin{tabular}{|c|c|c|c|c|c|c|c|c|c|c|c|c|c|c|c|c|c|c|}
\hline 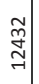 & 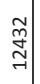 & 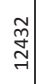 & 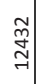 & $\underset{\widetilde{N}}{\underset{\sim}{\sim}}$ & 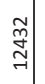 & $\underset{\widetilde{N}}{\underset{\sim}{\sim}}$ & $\begin{array}{l}\tilde{\sim} \\
\underset{\sim}{\sim} \\
\mid\end{array}$ & 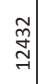 & 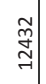 & 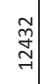 & $\begin{array}{l}\tilde{N} \\
\underset{\sim}{\sim}\end{array}$ & $\underset{\widetilde{f}}{\widetilde{ন}}$ & $\begin{array}{l}\tilde{g} \\
\underset{\sim}{\sim}\end{array}$ & $\infty$ & $\infty$ & $\infty$ & $\infty$ & 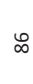 \\
\hline in & $\nabla$ & 0 & -1 & 0 & 0 & 0 & 0 & 0 & 0 & 0 & 0 & 0 & 0 & 0 & o & 0 & 0 & $\circ$ \\
\hline 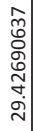 & 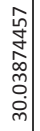 & 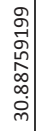 & 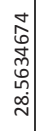 & 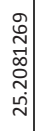 & 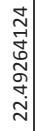 & $\begin{array}{l}\vec{b} \\
0 \\
0 \\
0 \\
0 \\
0 \\
0 \\
-1\end{array}$ & 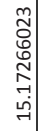 & 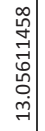 & 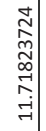 & 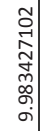 & 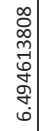 & 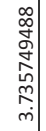 & 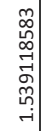 & 0 & $\begin{array}{l}\stackrel{\sim}{N} \\
0 \\
0\end{array}$ & 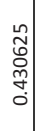 & 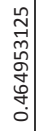 & $\begin{array}{l}0 \\
\stackrel{0}{0} \\
0 \\
0 \\
\circ \\
0\end{array}$ \\
\hline (6) & 영 & in & $g$ & $g$ & $\stackrel{m}{m}$ & $\stackrel{\sim}{\infty}$ & $\stackrel{\sim}{\sim}$ & $\tilde{\sim}$ & 9 & Q & in & $\pi$ & $\rightarrow$ & $\rightarrow$ & $\rightarrow$ & $\rightarrow$ & $\rightarrow$ & - \\
\hline 竺 & 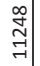 & \begin{tabular}{l}
$\mathbb{J}$ \\
\multirow{Z}{*}{}
\end{tabular} & 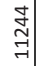 & $\begin{array}{l}\underset{J}{\mathbb{J}} \\
\mid\end{array}$ & 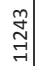 & 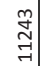 & $\begin{array}{l}\stackrel{m}{\Xi} \\
\exists\end{array}$ & $\underset{\Xi}{\Xi}$ & $\underset{\Xi}{\Xi}$ & 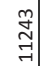 & 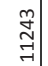 & 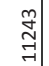 & 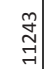 & $\stackrel{\llcorner}{\infty}$ & $\infty$ & $\infty$ & $\stackrel{\infty}{\infty}$ & $\infty$ \\
\hline 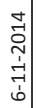 & 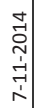 & $\begin{array}{l}\vec{d} \\
\stackrel{1}{2} \\
\stackrel{7}{7} \\
\infty\end{array}$ & 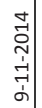 & 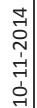 & 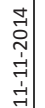 & 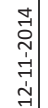 & 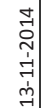 & 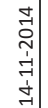 & 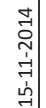 & 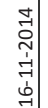 & 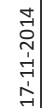 & $\begin{array}{l}0 \\
0 \\
0 \\
-1 \\
\Delta \\
0\end{array}$ & $\begin{array}{l}0 \\
0 \\
-1 \\
\vec{a} \\
\Delta\end{array}$ & 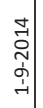 & 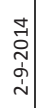 & 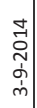 & 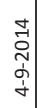 & ָิ \\
\hline 0 & 0 & 0 & 0 & 0 & 0 & 0 & 0 & 0 & 0 & 0 & 0 & 0 & 0 & - & $\rightarrow$ & $\rightarrow$ & $\rightarrow$ & 7 \\
\hline 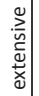 & 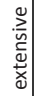 & 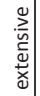 & 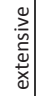 & 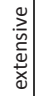 & 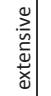 & 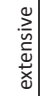 & 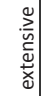 & 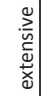 & 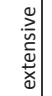 & 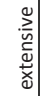 & $\begin{array}{l}\stackrel{0}{u} \\
\bar{u} \\
\vec{v} \\
\vec{v}\end{array}$ & 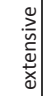 & 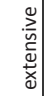 & 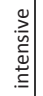 & 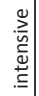 & 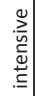 & 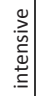 & $\sum_{\bar{c}}^{0}$ \\
\hline$\sigma$ & $\nabla$ & $\nabla$ & $\sigma$ & $\nabla$ & $\nabla$ & $\nabla$ & $\nabla$ & $\sigma$ & $\nabla$ & $\nabla$ & $\nabla$ & $\diamond$ & $\diamond$ & $N$ & $\sim$ & $\sim$ & $\sim$ & $r$ \\
\hline 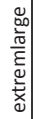 & 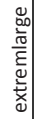 & 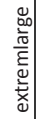 & 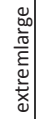 & 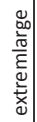 & 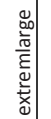 & 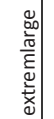 & 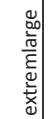 & 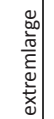 & 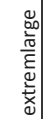 & 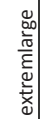 & 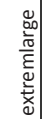 & 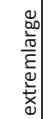 & 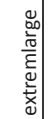 & \begin{tabular}{c|c}
$\stackrel{0}{0}$ \\
$\frac{0}{\sigma 0}$
\end{tabular} & 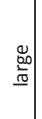 & $\begin{array}{l}\frac{\mathrm{d}}{\mathrm{d}} \\
\frac{\mathrm{w}}{\mathrm{a}}\end{array}$ & $\begin{array}{l}\frac{\mathrm{a}}{\mathrm{a}} \\
\frac{\mathrm{w}}{\mathrm{a}}\end{array}$ & $\begin{array}{l}\text { 品 } \\
\text { a. }\end{array}$ \\
\hline r & r & $r$ & $r$ & $\wedge$ & $\wedge$ & $\wedge$ & $\wedge$ & N & ^ & $\wedge$ & $\Lambda$ & $N$ & $\wedge$ & 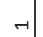 & $\rightarrow$ & $\rightarrow$ & $\rightarrow$ & . \\
\hline$\sum^{\circ}$ & $\stackrel{\text { and }}{\stackrel{0}{2}}$ & $\sum^{\frac{\pi}{0}}$ & $\frac{\mathrm{m}}{2}$ & $\stackrel{\frac{\pi}{0}}{\stackrel{0}{0}}$ & $\begin{array}{l}\frac{\pi}{0} \\
\stackrel{0}{2}\end{array}$ & 党| & 登| & 吾| & \begin{tabular}{l|l}
$\frac{\pi}{2}$ \\
$\stackrel{0}{\circ}$
\end{tabular} & 韋 & 离 & 韋 & $\begin{array}{l}\stackrel{8}{\circ} \\
\stackrel{0}{2}\end{array}$ & 昜 & $\begin{array}{l}\text { ㅇํํ } \\
\text { | }\end{array}$ & $\begin{array}{l}\text { : } \\
\text { है }\end{array}$ & 总 & \\
\hline
\end{tabular}




\begin{tabular}{|c|c|c|c|c|c|c|c|c|c|c|c|c|c|c|c|c|c|c|c|}
\hline$\infty$ & $\infty$ & $\infty$ & $\infty$ & $\infty$ & $\infty$ & $\infty$ & $\infty$ & $\infty$ & $\infty$ & $\infty$ & $\begin{array}{l}0 \\
\infty\end{array}$ & $\infty$ & $\infty$ & $\infty$ & $\infty$ & $\stackrel{\infty}{\infty}$ & $\Phi$ & 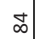 & \\
\hline 0 & 0 & 0 & 0 & 0 & 0 & 0 & 0 & 0 & 0 & 0 & $\pi$ & $m$ & 0 & 0 & 0 & 0 & 0 & $\rightarrow$ & \\
\hline 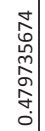 & 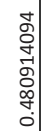 & 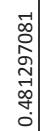 & 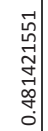 & 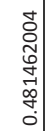 & 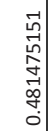 & 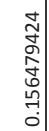 & $\begin{array}{l}m \\
0 \\
0 \\
0 \\
0 \\
0 \\
0 \\
0 \\
0 \\
0\end{array}$ & 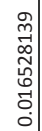 & 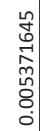 & 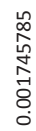 & $\begin{array}{l}\infty \\
\stackrel{n}{0} \\
\hat{L} \\
0 \\
0 \\
0\end{array}$ & 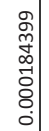 & $\begin{array}{c}n \\
0 \\
0 \\
0 \\
0 \\
0 \\
0 \\
0\end{array}$ & 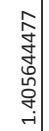 & 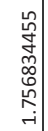 & 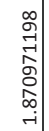 & 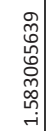 & 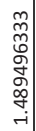 & \\
\hline
\end{tabular}

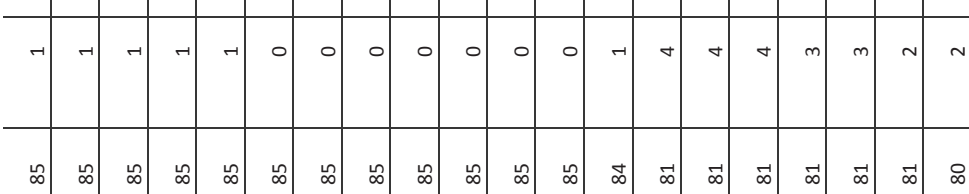

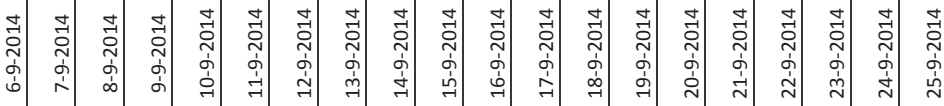

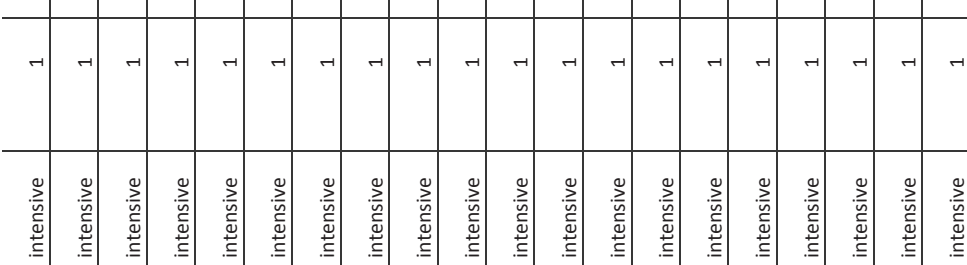

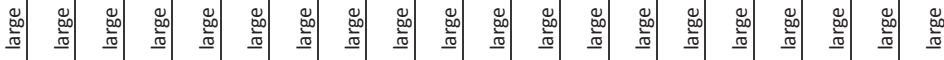

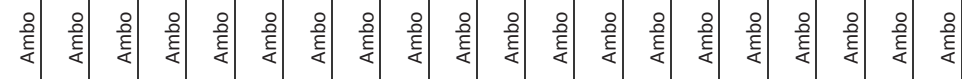




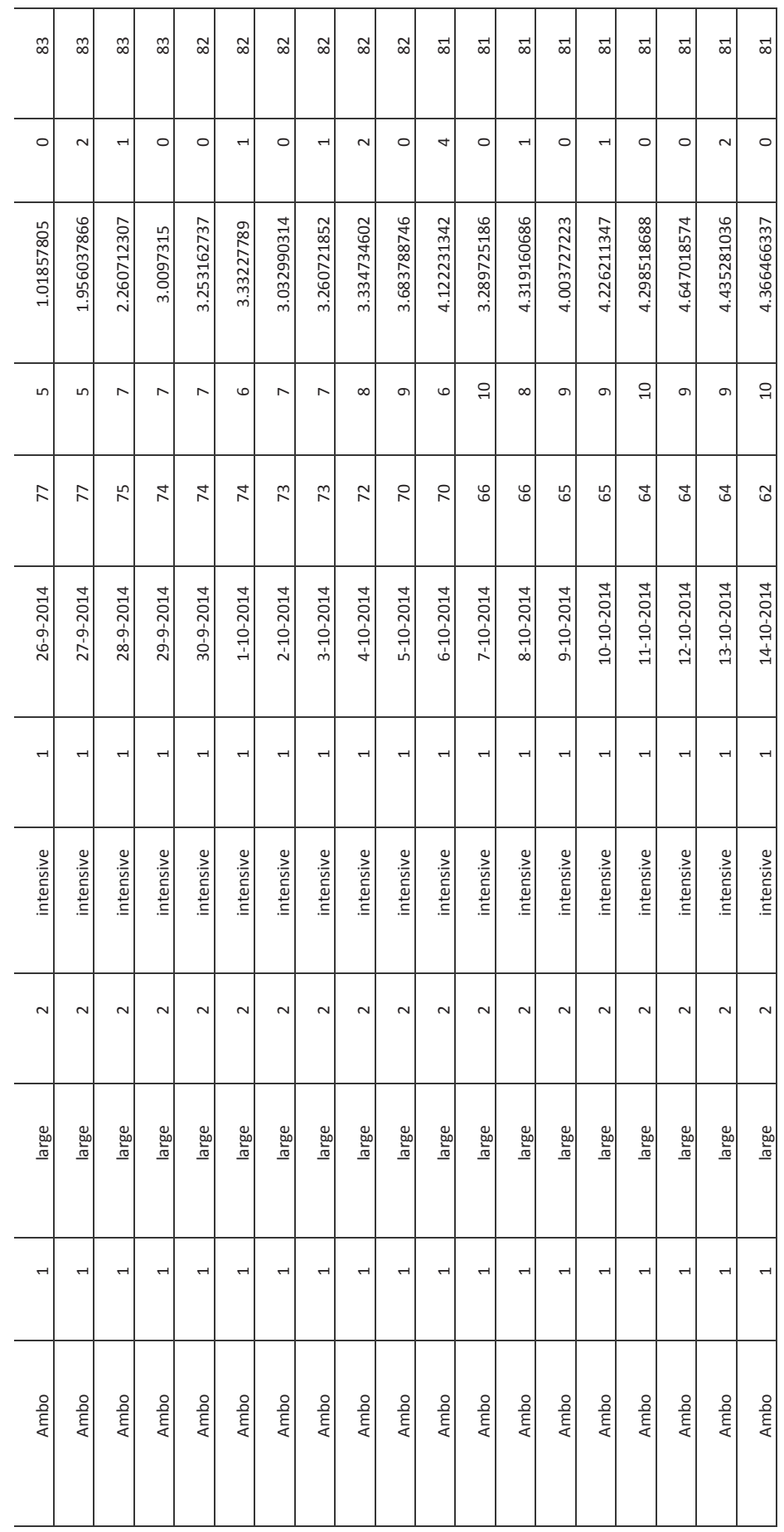




\begin{tabular}{|c|c|c|c|c|c|c|c|c|c|c|c|c|c|c|c|c|c|c|c|}
\hline$\vec{\infty}$ & $\vec{\infty}$ & $\vec{\infty}$ & $\infty$ & $\triangleright$ & $\infty$ & $\infty$ & $\triangleright$ & $\triangleright$ & 요 & 요 & 은 & ㅇํ & 은 & 은 & 우 & 우 & 요 & 은 & in \\
\hline 0 & 0 & 0 & 0 & 0 & 0 & 0 & 0 & 0 & 0 & 0 & 0 & 0 & $\rightarrow$ & 0 & 0 & $\neg$ & -1 & 0 & \\
\hline $\begin{array}{l}0 \\
0 \\
n \\
0 \\
0 \\
0 \\
0 \\
\dot{\sigma}\end{array}$ & 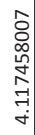 & 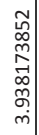 & 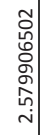 & $\begin{array}{l}m \\
\overrightarrow{0} \\
0 \\
0 \\
0 \\
m \\
0 \\
0 \\
-1\end{array}$ & 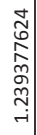 & 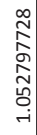 & 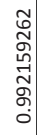 & 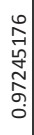 & 0 & $\begin{array}{l}\stackrel{\sim}{\cong} \\
\stackrel{m}{0}\end{array}$ & 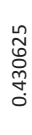 & $\begin{array}{l}\stackrel{2}{N} \\
0 \\
0 \\
0 \\
0 \\
0 \\
0 \\
0\end{array}$ & 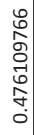 & 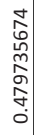 & 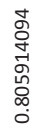 & $\begin{array}{l}\text { o̊ } \\
\text { స్. } \\
\text { హ. } \\
\text { శ్ }\end{array}$ & $\begin{array}{l}0 \\
0 \\
b \\
\hat{\sigma} \\
\hat{\sigma} \\
\sigma \\
0 \\
0\end{array}$ & 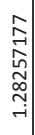 & \\
\hline
\end{tabular}

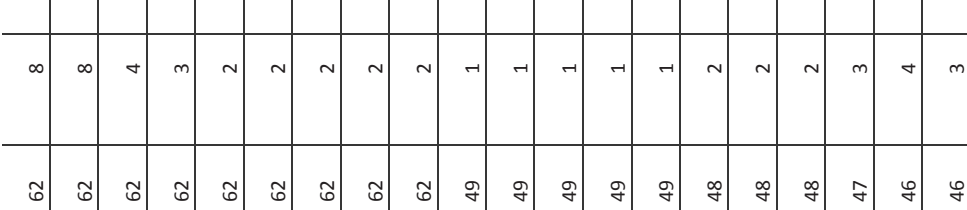

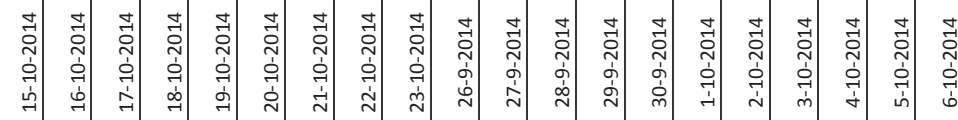

\begin{tabular}{|c|c|c|c|c|c|c|c|c|c|c|c|c|c|c|c|c|c|c|c|}
\hline - & -1 & $\rightarrow$ & -1 & -1 & -1 & -1 & -1 & -1 & $\neg$ & -1 & -1 & -1 & -1 & -1 & $r$ & -1 & -1 & -1 & -1 \\
\hline$\stackrel{\unlhd}{.}$ & 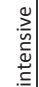 & 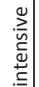 & 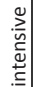 & 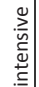 & 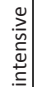 & 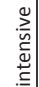 & 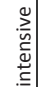 & 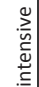 & 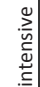 & 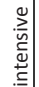 & 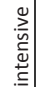 & 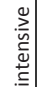 & 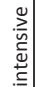 & 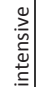 & 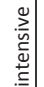 & 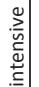 & 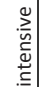 & 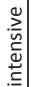 & \\
\hline
\end{tabular}

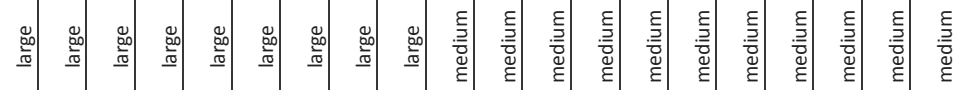

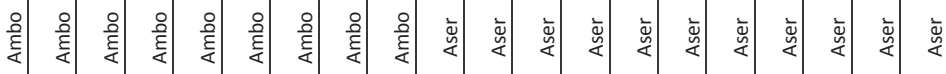




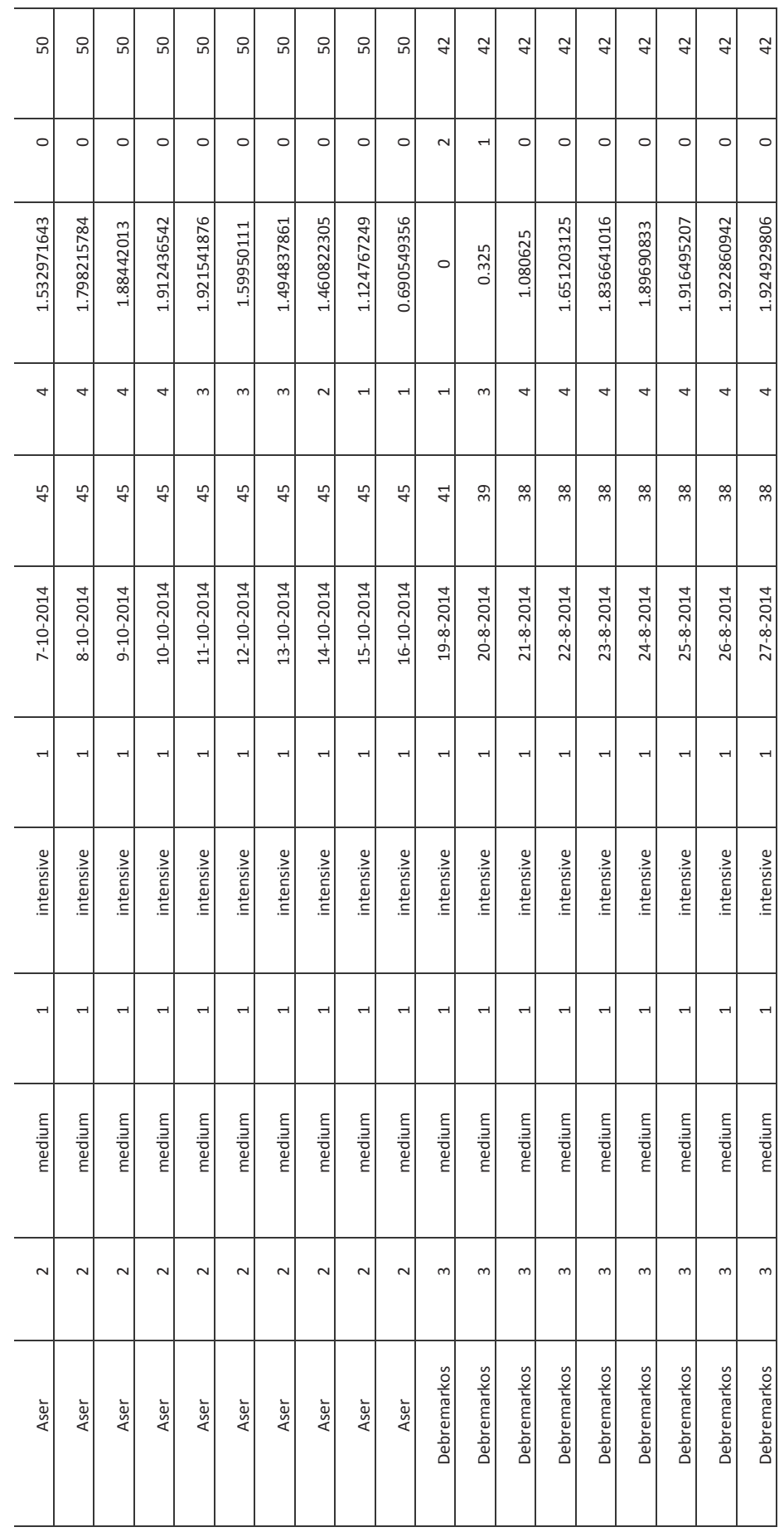




\begin{tabular}{|c|c|c|c|c|c|c|c|c|c|c|c|c|c|c|c|c|c|c|c|}
\hline$\approx$ & $\mathcal{F}$ & $\mathcal{f}$ & $\mathcal{7}$ & $\mathcal{\gamma}$ & $\mathcal{7}$ & $\mathcal{F}$ & F & $\mathcal{\gamma}$ & $\mathcal{F}$ & $\mathcal{\gamma}$ & $\mathcal{7}$ & $q$ & $\mathcal{F}$ & $\underset{ซ}{2}$ & $\stackrel{2}{\mathscr{\gamma}}$ & ఫิ & శి & ซे & \\
\hline- & 0 & 0 & 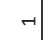 & 。 & 0 & 0 & 0 & $\circ$ & 0 & 0 & 0 & 0 & 0 & 0 & 0 & 0 & 0 & 0 & \\
\hline 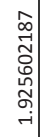 & 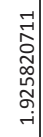 & 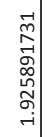 & 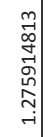 & 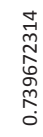 & $\begin{array}{c}\text { D } \\
0 \\
0 \\
0 \\
0 \\
0 \\
0 \\
0 \\
0\end{array}$ & 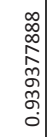 & $\begin{array}{l}\vec{~} \\
0 \\
0 \\
0 \\
0 \\
0 \\
0 \\
0\end{array}$ & 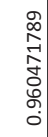 & 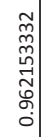 & 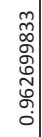 & 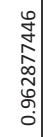 & $\begin{array}{l}\text { ज्ञ } \\
\hat{\Omega} \\
\hat{0} \\
\hat{0} \\
0\end{array}$ & 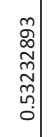 & 0 & $\begin{array}{c}\tilde{\tilde{n}} \\
\mathrm{O}\end{array}$ & 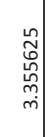 & 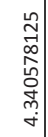 & 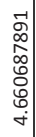 & \\
\hline
\end{tabular}

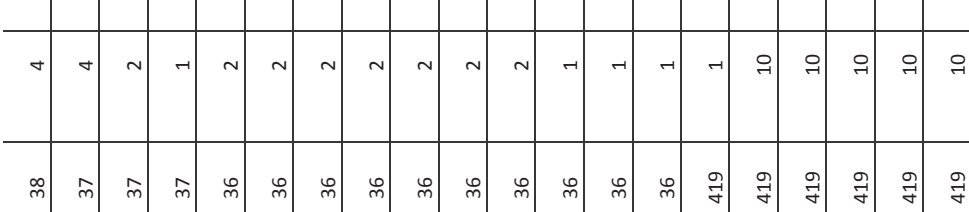

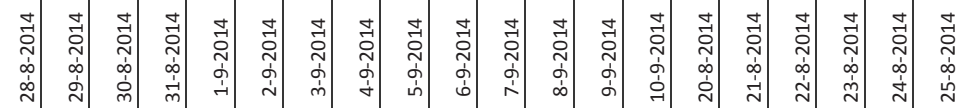

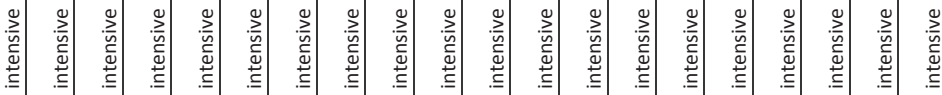

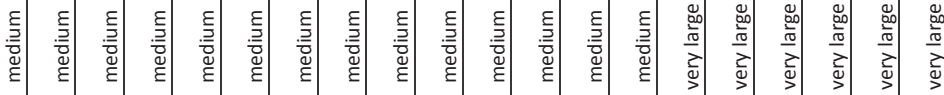

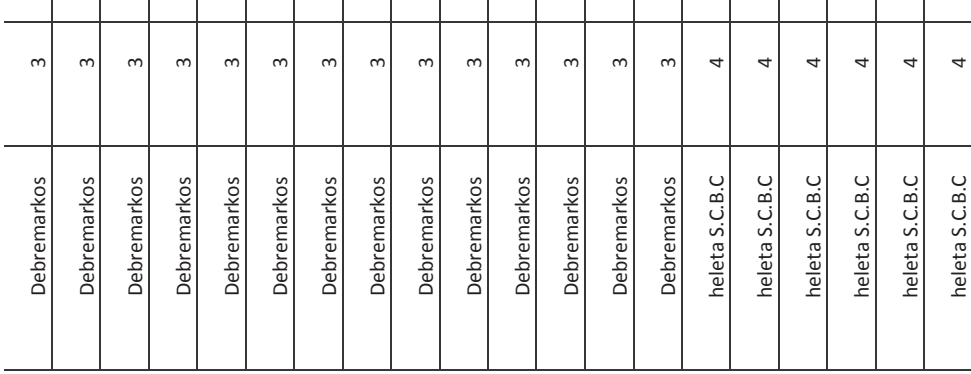




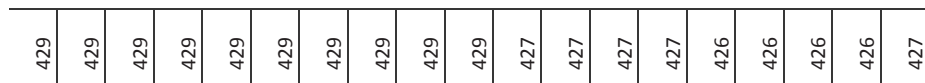

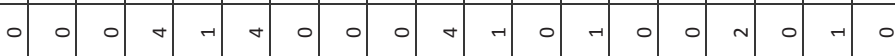

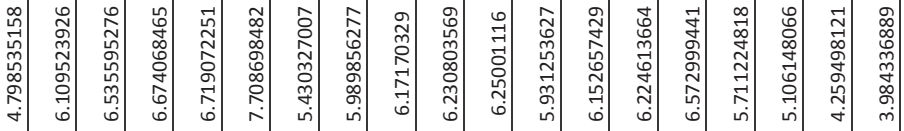

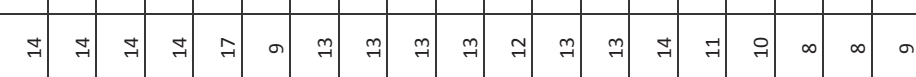

岁

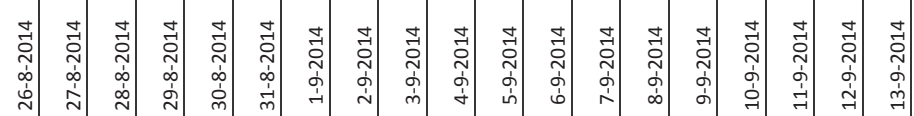

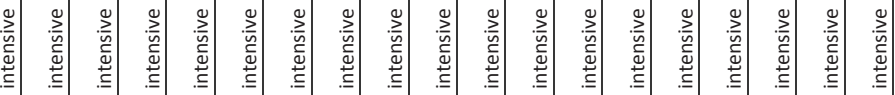

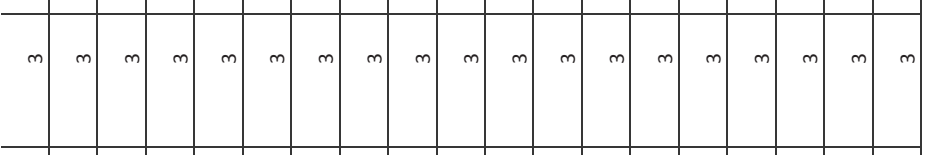

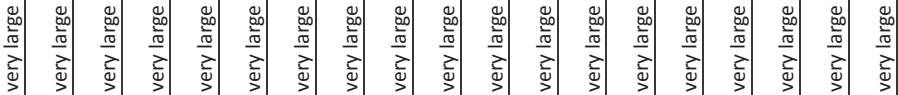

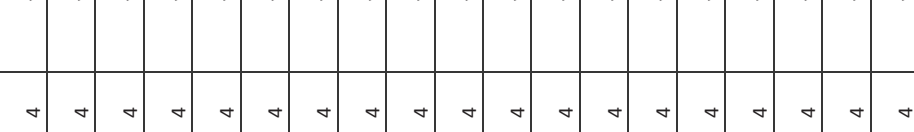

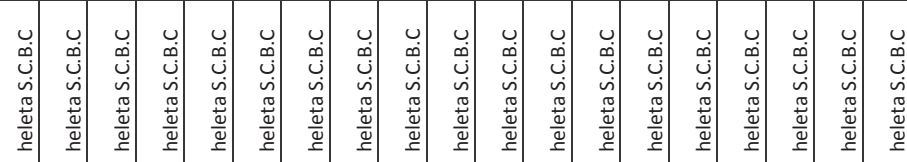




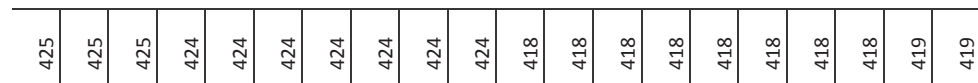

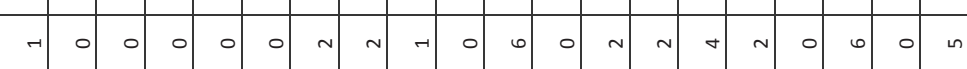

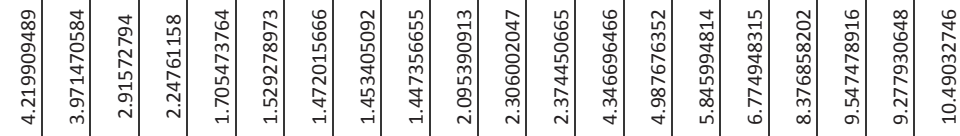

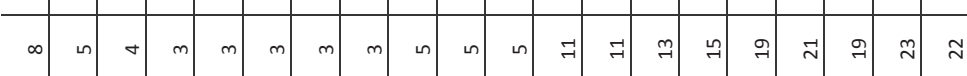

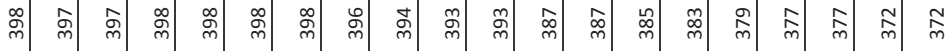

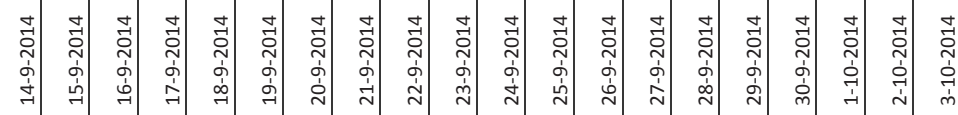

\begin{tabular}{|c|c|c|c|c|c|c|c|c|c|c|c|c|c|c|c|c|c|c|c|}
\hline$\rightarrow$ & -1 & $\rightarrow$ & -1 & $\rightarrow$ & $\rightarrow$ & $r$ & 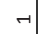 & $\rightarrow$ & -1 & $\rightarrow$ & -1 & -1 & -1 & -1 & $\rightarrow$ & $\rightarrow$ & -1 & -1 & -1 \\
\hline$\stackrel{\searrow}{\geq}$ & 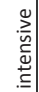 & 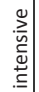 & 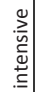 & 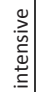 & 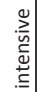 & 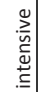 & 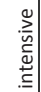 & 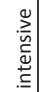 & 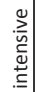 & 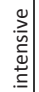 & 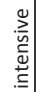 & 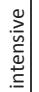 & 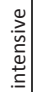 & 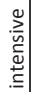 & 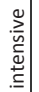 & 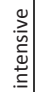 & 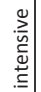 & 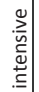 & $\sum_{\bar{c}}^{\frac{m}{m}}$ \\
\hline
\end{tabular}

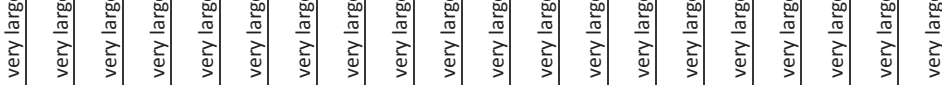

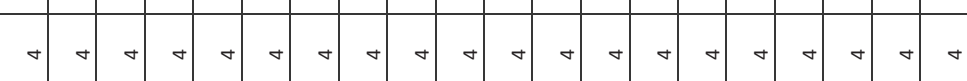

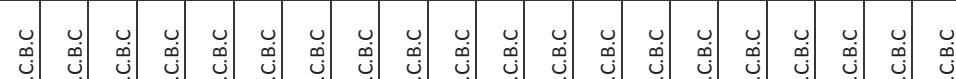

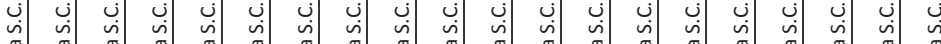

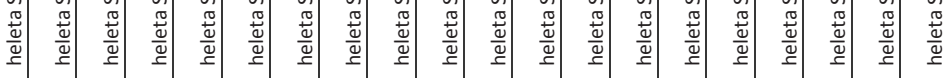




\begin{tabular}{|c|c|c|c|c|c|c|c|c|c|c|c|c|c|c|c|c|c|c|}
\hline$\stackrel{\infty}{\vec{f}}$ & $\stackrel{\infty}{\vec{f}}$ & $\stackrel{\infty}{\exists}$ & $\stackrel{\infty}{\vec{\gamma}}$ & $\stackrel{\infty}{\vec{\gamma}}$ & $\stackrel{\infty}{\exists}$ & $\stackrel{\vec{f}}{\vec{f}}$ & $\stackrel{\vartheta}{\sigma}$ & $\vec{f}$ & $\vec{g}$ & $\stackrel{m}{\vec{\gamma}}$ & $\stackrel{m}{\vec{q}}$ & $\vec{q}$ & $\stackrel{m}{\gamma}$ & $\stackrel{m}{f}$ & $\begin{array}{l}m \\
\vec{\gamma}\end{array}$ & $\mathcal{F}$ & $z$ & $\overline{7}$ \\
\hline 0 & ० & 0 & 0 & 0 & $\nabla$ & $m$ & 0 & 0 & o & 0 & 0 & 0 & 0 & 0 & 0 & 0 & $\rightarrow$ & \\
\hline 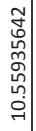 & 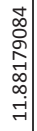 & $\begin{array}{l} \\
\tilde{0} \\
0 \\
0 \\
0 \\
0 \\
0 \\
0 \\
0\end{array}$ & 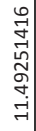 & $\begin{array}{l}-1 \\
0 \\
0 \\
-1 \\
-1\end{array}$ & 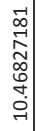 & 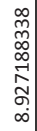 & $\begin{array}{l}\vec{\delta} \\
0 \\
\tilde{\omega} \\
\sigma \\
\sigma \\
\sigma\end{array}$ & $\begin{array}{l}\text { N } \\
\tilde{N} \\
0 \\
0 \\
0 \\
0\end{array}$ & 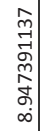 & 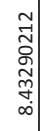 & $\begin{array}{c}0 \\
0 \\
0 \\
0 \\
0 \\
0 \\
0 \\
0 \\
0\end{array}$ & 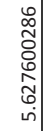 & $\begin{array}{l}m \\
o \\
o \\
\hat{o} \\
\hat{h} \\
\dot{q} \\
\dot{n}\end{array}$ & 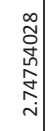 & 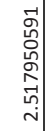 & 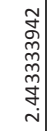 & 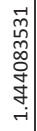 & \\
\hline
\end{tabular}

\begin{tabular}{|c|c|c|c|c|c|c|c|c|c|c|c|c|c|c|c|c|c|c|c|}
\hline$\stackrel{\mathbb{N}}{ }$ & 기 & $\stackrel{\mathfrak{N}}{*}$ & $\sim$ & $\vec{N}$ & F & i & $\approx$ & $\exists$ & न & 7 & $\exists$ & in & in & in & in & $\sim$ & 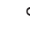 & & \\
\hline
\end{tabular}

自

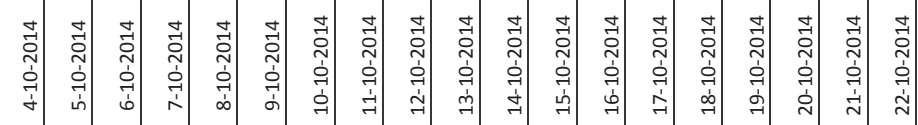

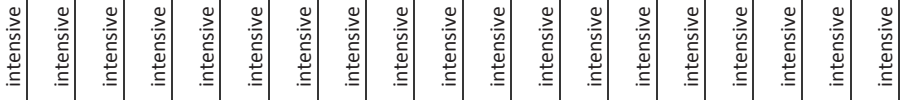

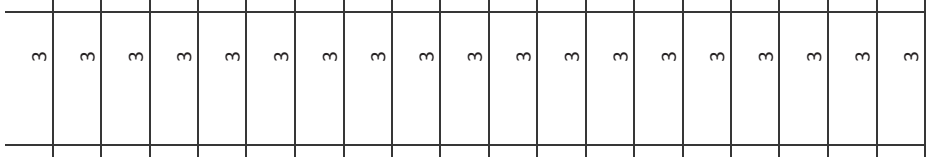

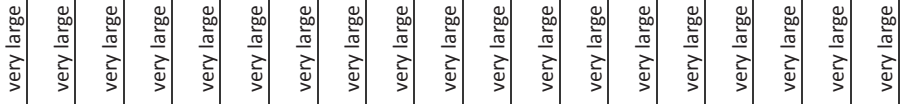

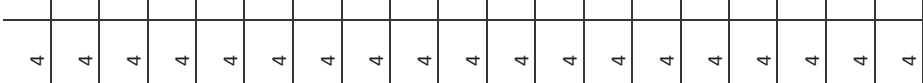

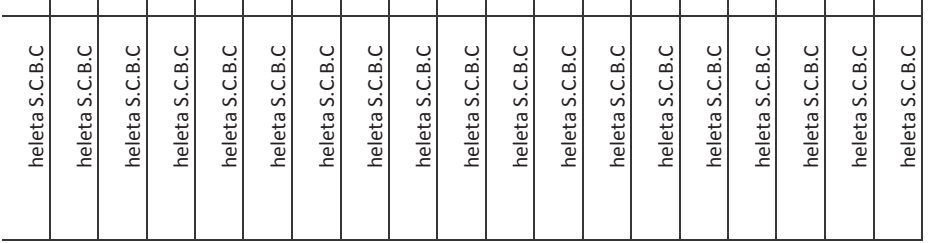




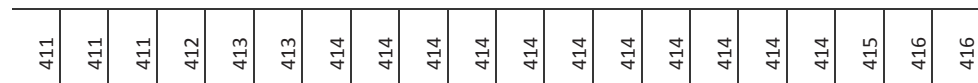

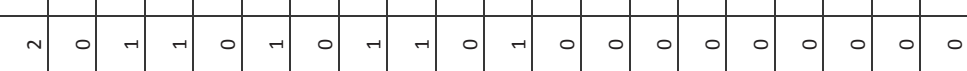

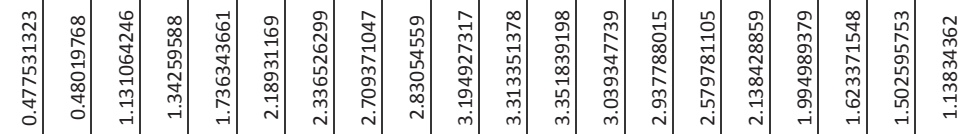

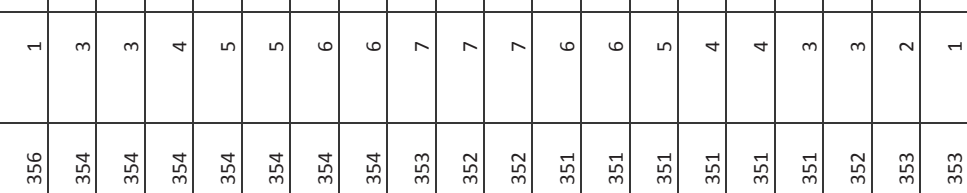

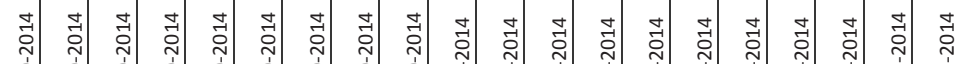

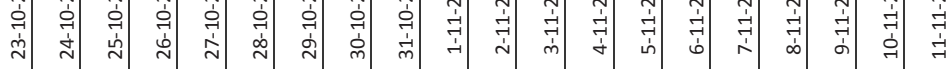

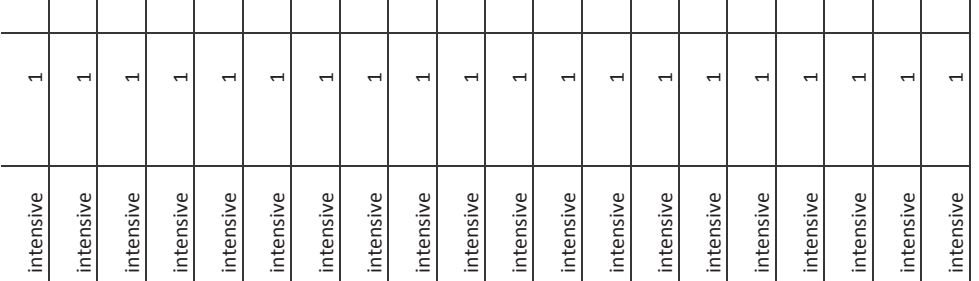

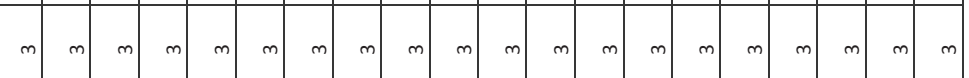

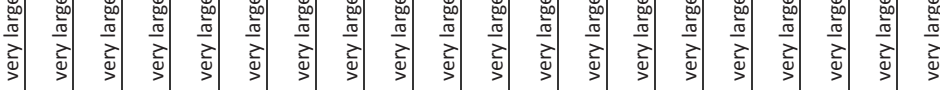

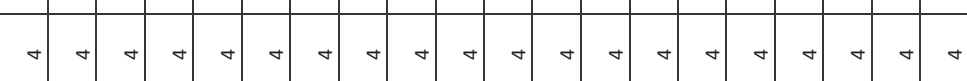

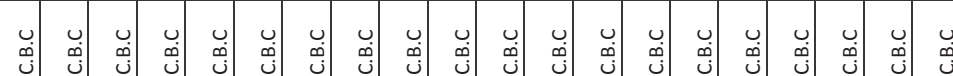

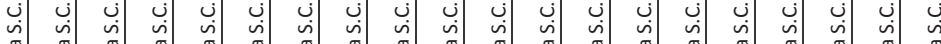

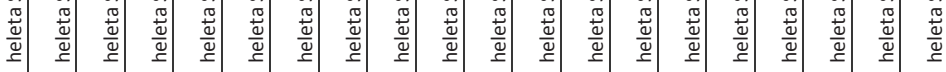




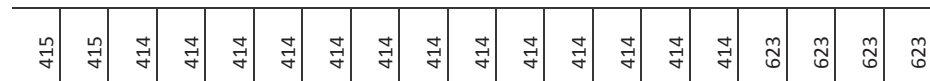

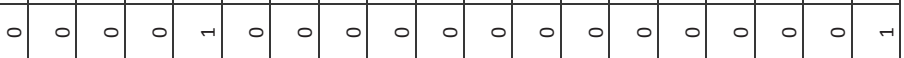

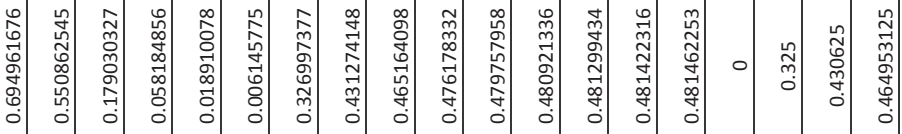

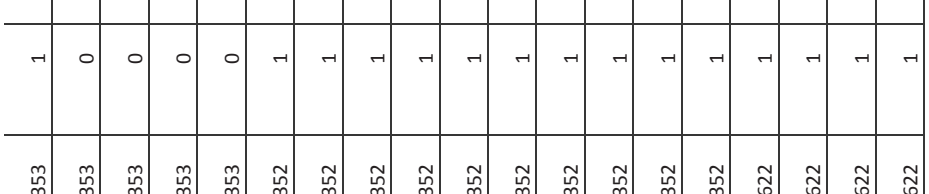

ஸू

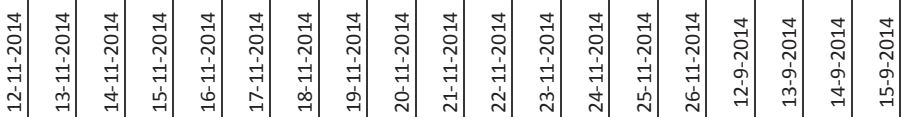

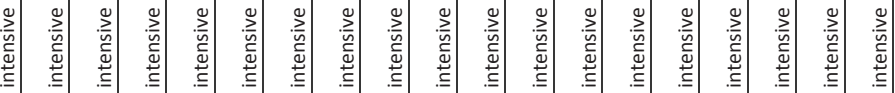

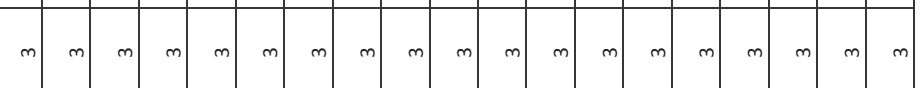

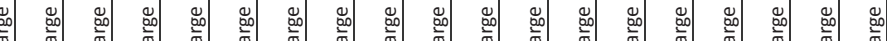

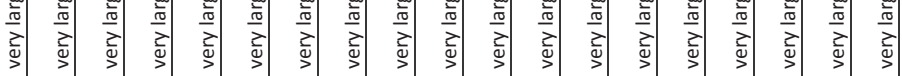

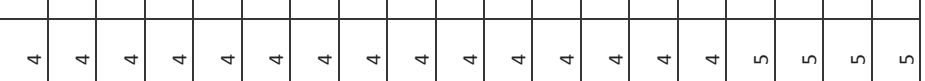

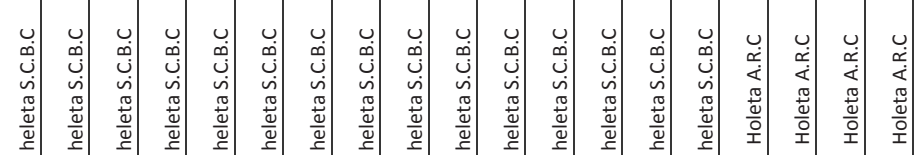




\begin{tabular}{|c|c|c|c|c|c|c|c|c|c|c|c|c|c|c|c|c|c|c|c|}
\hline$\widetilde{\widetilde{\sigma}}$ & 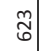 & $\underset{\widetilde{\sigma}}{ }$ & $\underset{\widetilde{\sigma}}{\widetilde{2}}$ & 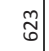 & $\underset{\widetilde{\sigma}}{\mathscr{2}}$ & $\stackrel{\widetilde{\sigma}}{0}$ & శ్ర & $\tilde{\widetilde{\sigma}}$ & $\tilde{్}$ & $\widetilde{\widetilde{\sigma}}$ & $\underset{్ ర ్}{0}$ & జ్ర & $\begin{array}{l}\mathfrak{\sigma} \\
\mid\end{array}$ & $\stackrel{\widetilde{\sigma}}{ }$ & $\tilde{\widetilde{\sigma}}$ & $\mid$ & $\stackrel{\infty}{\overrightarrow{0}}$ & $\underset{ర}{\infty}$ & \\
\hline-1 & 0 & 0 & 0 & 0 & 0 & 0 & -1 & 0 & 0 & 0 & ○ & 0 & 0 & 0 & -1 & -1 & 0 & 0 & \\
\hline $\begin{array}{l}0 \\
0 \\
0 \\
0 \\
0 \\
0 \\
0 \\
0\end{array}$ & 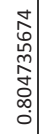 & 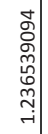 & 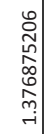 & 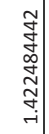 & 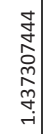 & 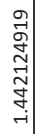 & 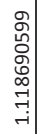 & 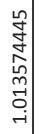 & 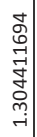 & $\begin{array}{c}\overrightarrow{0} \\
\infty \\
0 \\
0 \\
0 \\
0 \\
m \\
\vec{i}\end{array}$ & 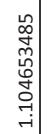 & 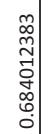 & 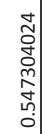 & $\begin{array}{l}\infty \\
0 \\
0 \\
0 \\
\infty \\
\tilde{D} \\
0 \\
0 \\
0\end{array}$ & $\begin{array}{l}\infty \\
0 \\
0 \\
0 \\
\tilde{p} \\
0 \\
0 \\
o \\
0\end{array}$ & 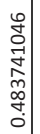 & 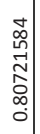 & 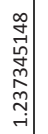 & \\
\hline$\sim$ & $m$ & $\mathrm{~m}$ & $m$ & $\mathrm{~m}$ & $\mathrm{~m}$ & $\sim$ & $\sim$ & $m$ & $m$ & $\sim$ & I & | & $\rightarrow$ & $\rightarrow$ & $\rightarrow$ & $\sim$ & $m$ & $\sim$ & \\
\hline$\vec{ర}$ & ปุำ & ণิ & ఫิ & ఫิ & రి & ¿ุర & ธิธ్ & $\vec{\emptyset}$ & :ै & $\vec{\emptyset}$ & $\stackrel{\overrightarrow{0}}{0}$ & $\overrightarrow{6}$ & $\overrightarrow{\mathrm{b}}$ & 잉 & वे & $\mid$\begin{tabular}{c}
$\infty$ \\
\hdashline \\
\end{tabular} & $\vec{b}$ & $\tilde{6}$ & \\
\hline $\begin{array}{l}0 \\
0 \\
T \\
o \\
\dot{\omega} \\
\text { | }\end{array}$ & 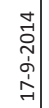 & 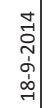 & 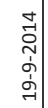 & $\begin{array}{l}5 \\
\vdots \\
0 \\
0 \\
\vdots \\
\dot{1}\end{array}$ & 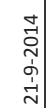 & 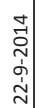 & $\begin{array}{l}\text { ज्ञ } \\
\hat{\sim} \\
\dot{\sim} \\
\dot{\sim}\end{array}$ & 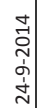 & 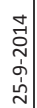 & 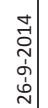 & 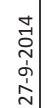 & $\begin{array}{l}d \\
0 \\
N \\
o \\
d \\
d\end{array}$ & 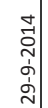 & 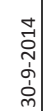 & $\begin{array}{l}5 \\
0 \\
T \\
0 \\
\vdots \\
-1\end{array}$ & 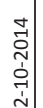 & $\begin{array}{l}0 \\
0 \\
0 \\
0 \\
0 \\
m\end{array}$ & 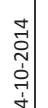 & \\
\hline- & -1 & |l & th & - & $\rightarrow$ & $\rightarrow$ & $\rightarrow$ & - & $\rightarrow$ & $\rightarrow$ & $\pi$ & $\rightarrow$ & -1 & $r$ & $r$ & -1 & $\rightarrow$ & $\rightarrow 1$ & \\
\hline 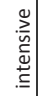 & 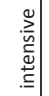 & 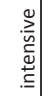 & 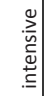 & 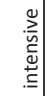 & : & 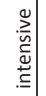 & 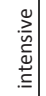 & 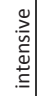 & : & 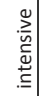 & 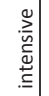 & 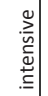 & 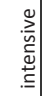 & 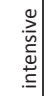 & 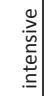 & 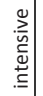 & 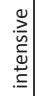 & 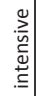 & \\
\hline
\end{tabular}

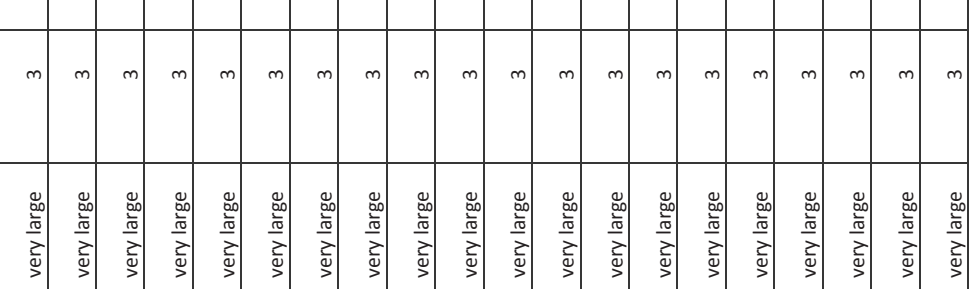

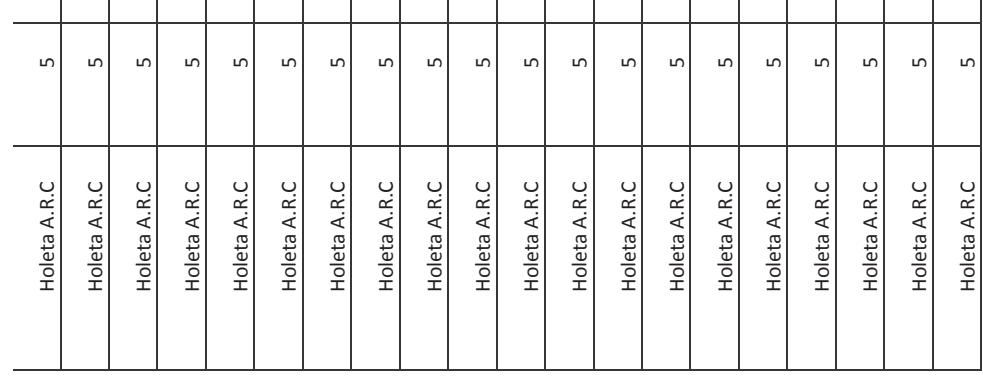




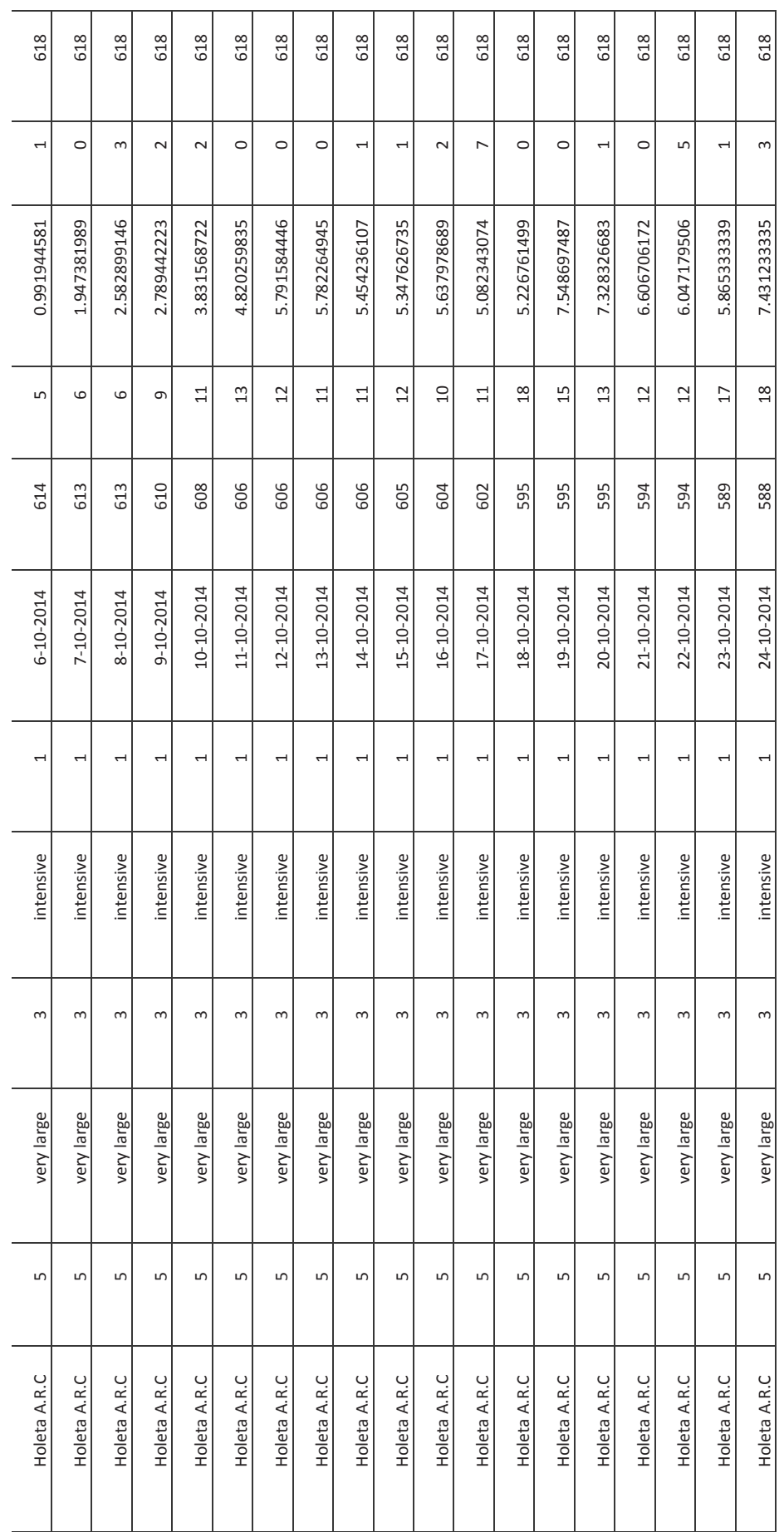




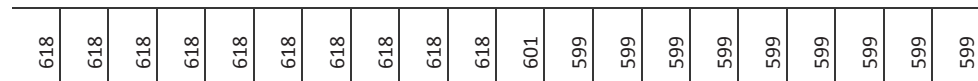

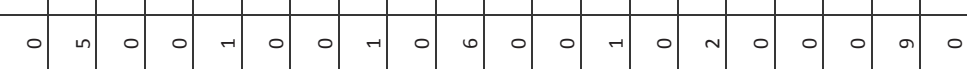

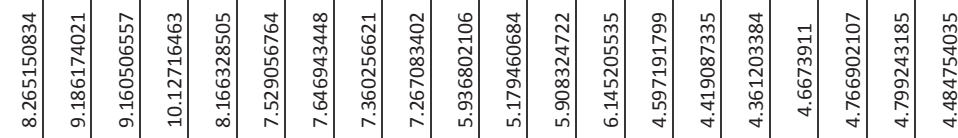

ㄴ

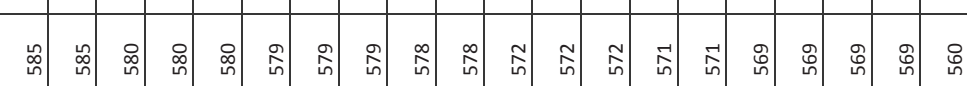

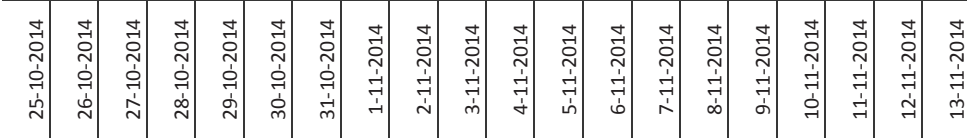

\begin{tabular}{|c|c|c|c|c|c|c|c|c|c|c|c|c|c|c|c|c|c|c|c|}
\hline & -1 & -1 & -1 & -1 & -1 & -1 & -1 & -1 & -1 & -1 & -7 & -1 & $\rightarrow$ & -7 & $\rightarrow$ & -1 & -1 & -1 & \\
\hline & & & & & & & & & & & & & & & & & & & \\
\hline$\stackrel{\nu}{=}$ & 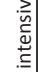 & 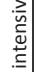 & 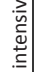 & 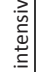 & 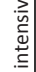 & 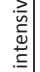 & 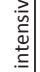 & 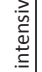 & & & & & 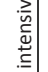 & & $\begin{array}{l}\vec{z} \\
\vec{u} \\
\stackrel{\vec{g}}{.}\end{array}$ & 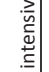 & 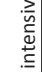 & 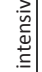 & \\
\hline
\end{tabular}

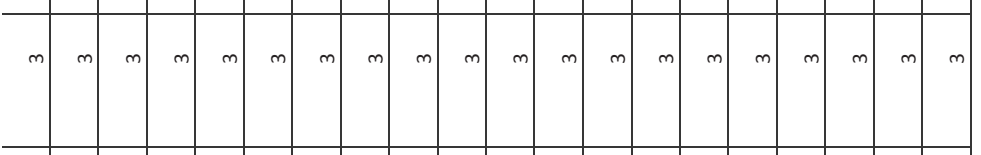

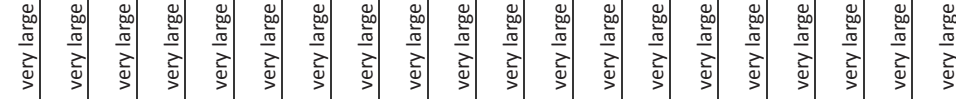

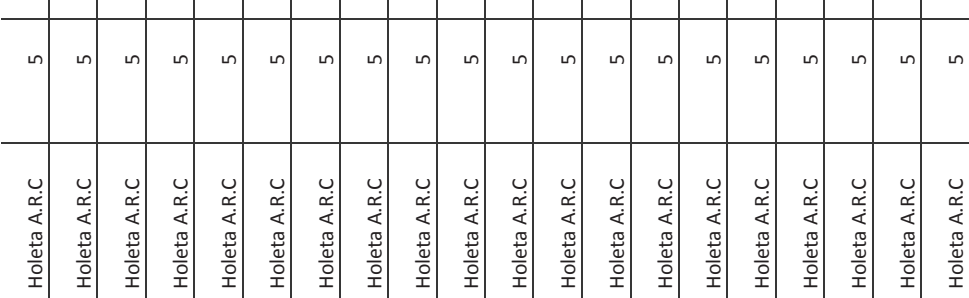




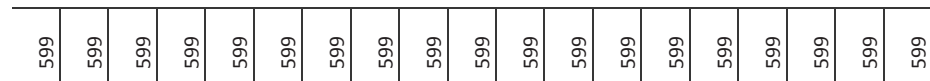

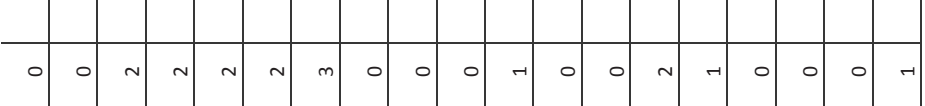

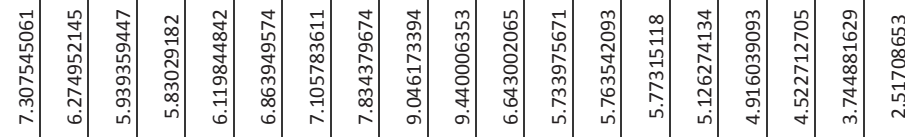

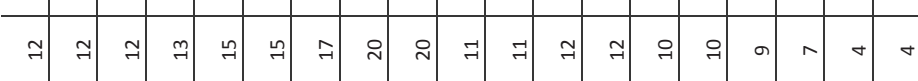

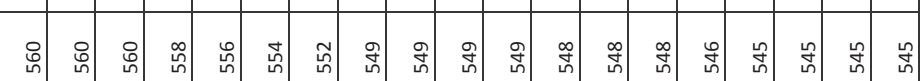

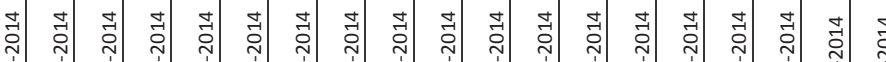

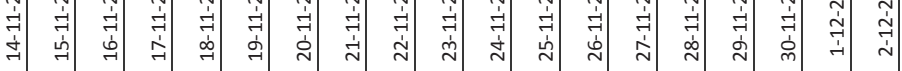

:

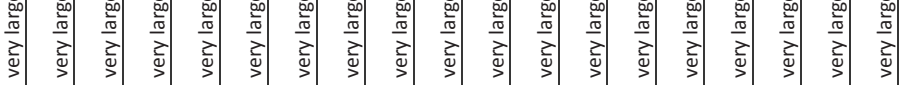

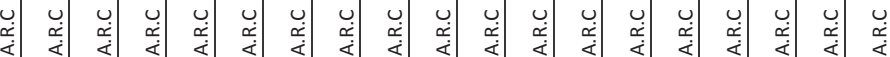

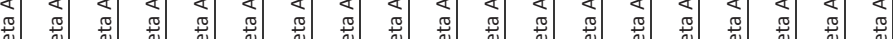

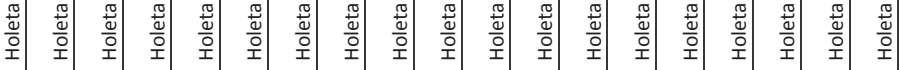




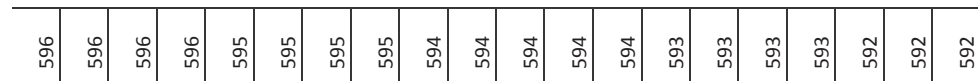

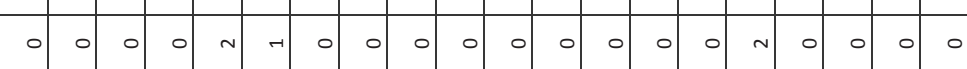

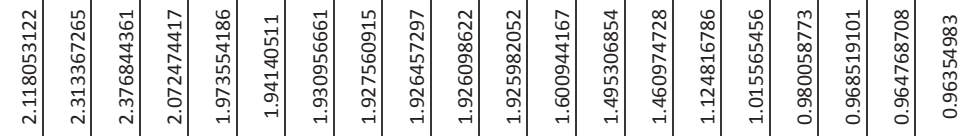

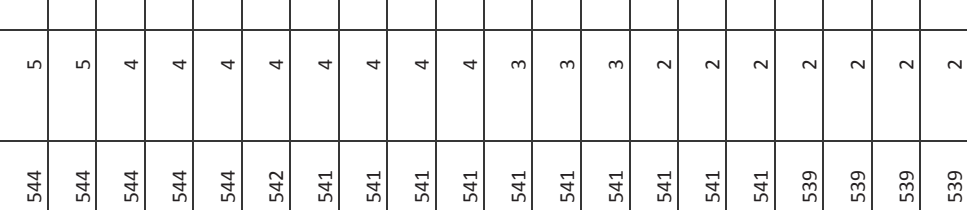

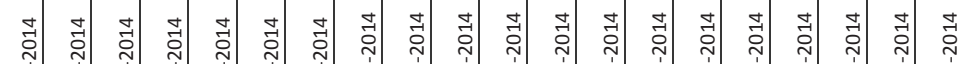

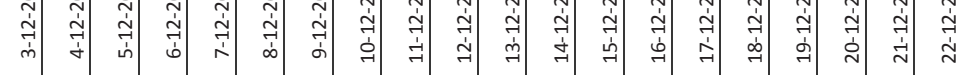

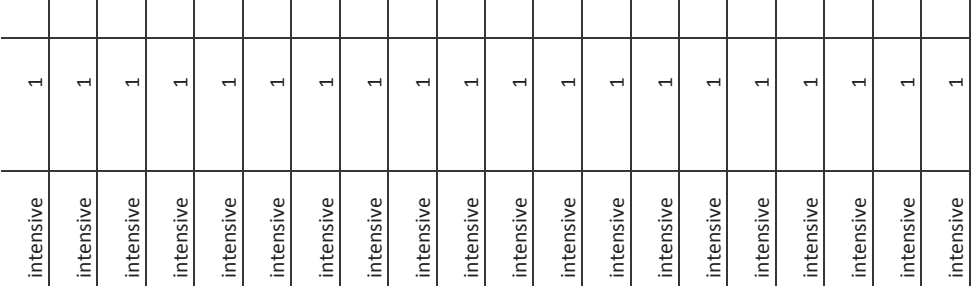

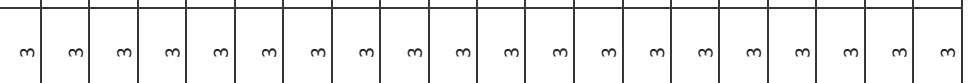

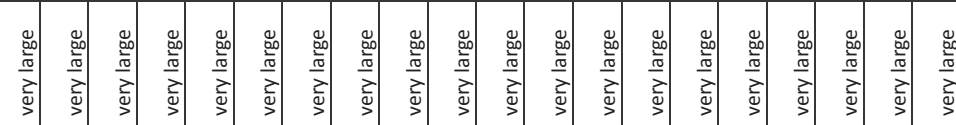

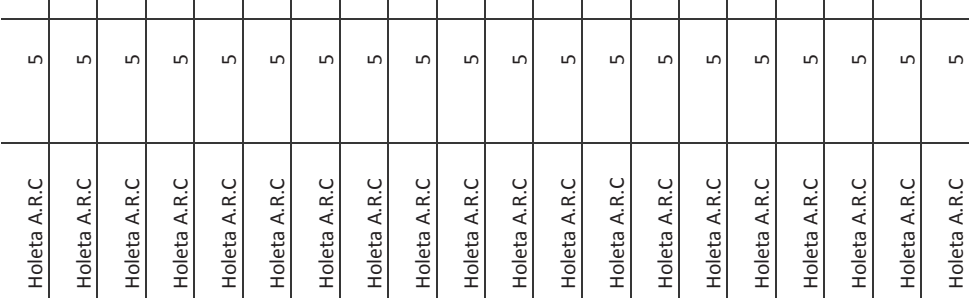




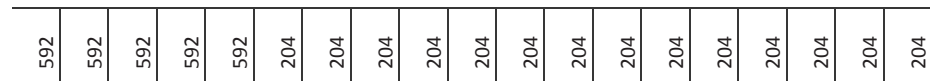

\begin{tabular}{llllllllllllllllllll|}
\hline & 0 & 0 & 0 & 0 & 0 & 0 & 0 & 0 & 0 & 0 & 4 & 0 & 0 & 0 & $n$ & 0 & 0 & 0
\end{tabular}

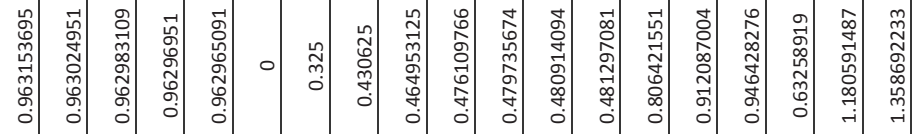

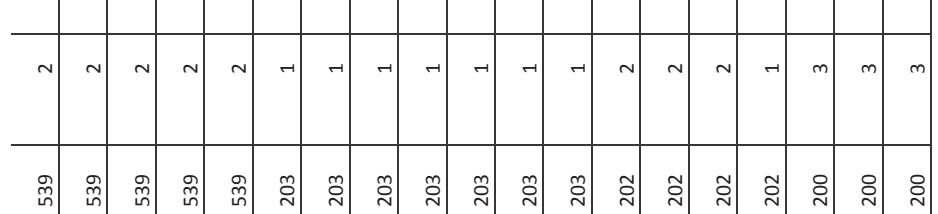

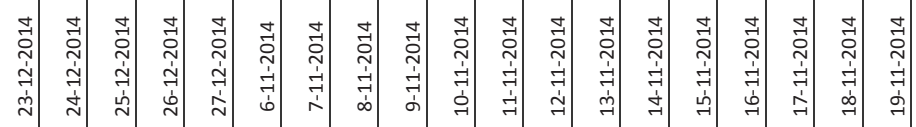

\begin{tabular}{|c|c|c|c|c|c|c|c|c|c|c|c|c|c|c|c|c|c|c|}
\hline-1 & $\rightarrow$ & $\rightarrow$ & -1 & $\neg$ & -1 & -1 & -1 & -1 & -1 & -1 & $\rightarrow$ & $\rightarrow$ & -1 & $\rightarrow$ & -1 & $\rightarrow$ & -1 & -1 \\
\hline 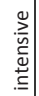 & 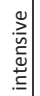 & 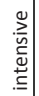 & 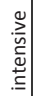 & 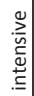 & $\begin{array}{l}\stackrel{0}{n} \\
\stackrel{2}{n} \\
\stackrel{0}{ \pm} \\
. \leq\end{array}$ & 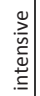 & 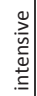 & 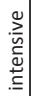 & 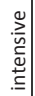 & 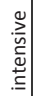 & 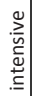 & 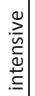 & 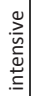 & $\begin{array}{l}\stackrel{0}{ \pm} \\
\stackrel{2}{0} \\
\stackrel{ \pm}{ \pm} \\
. \leq\end{array}$ & 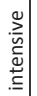 & 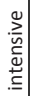 & 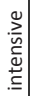 & 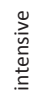 \\
\hline
\end{tabular}

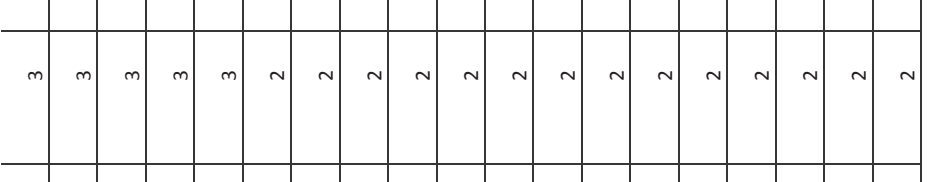

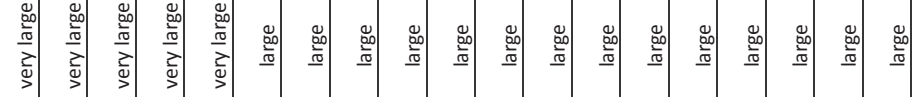

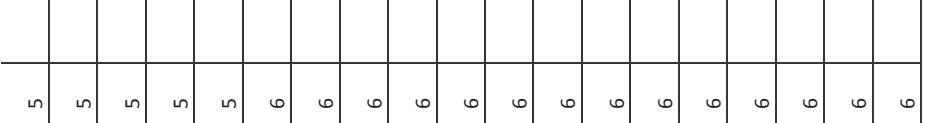

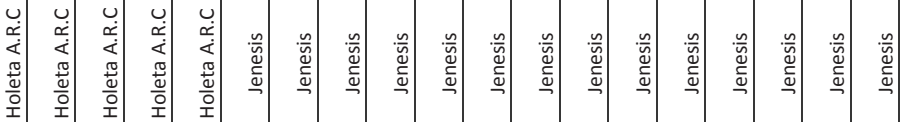


吾

\begin{tabular}{l|lll|llllllllllllllllll}
\hline 0 & 0 & 0 & 0 & -1 & 0 & 0 & 0 & 0 & 0 & 0 & -1 & 0 & 0 & 0 & 0 & 0 & 0 & 0 & 0
\end{tabular}

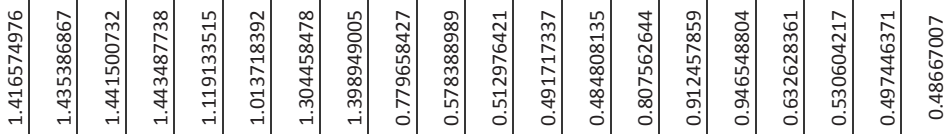

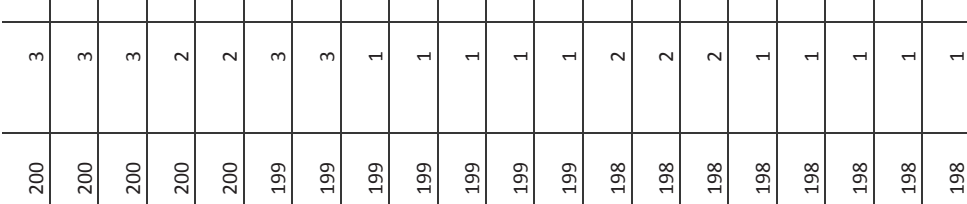

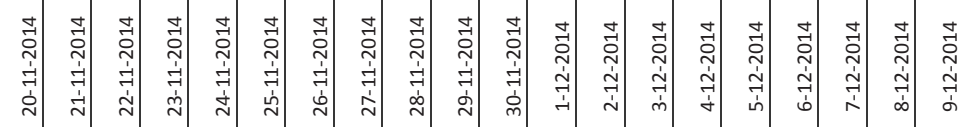

\begin{tabular}{|c|c|c|c|c|c|c|c|c|c|c|c|c|c|c|c|c|c|c|c|}
\hline & -1 & 7 & 7 & $\rightarrow$ & -1 & $r$ & -1 & $\rightarrow$ & $\rightarrow$ & $\rightarrow$ & -1 & 7 & -7 & 7 & $\rightarrow$ & -1 & $\rightarrow$ & $\pi$ & $\rightarrow$ \\
\hline$\frac{m}{n}$ & 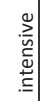 & 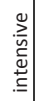 & 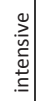 & 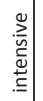 & 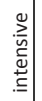 & 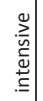 & 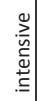 & 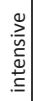 & 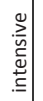 & 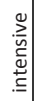 & 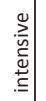 & 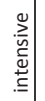 & 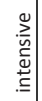 & 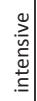 & 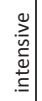 & 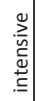 & 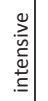 & 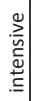 & $\sum_{\bar{m}}^{\bar{m}}$ \\
\hline
\end{tabular}

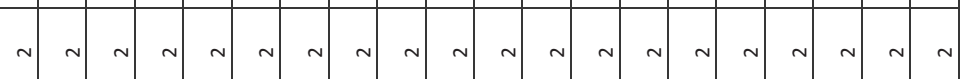

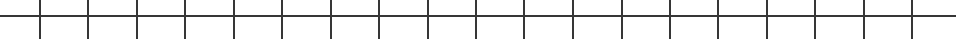

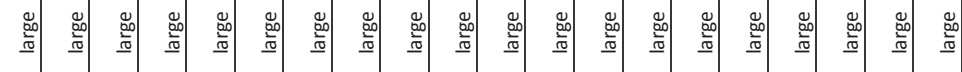

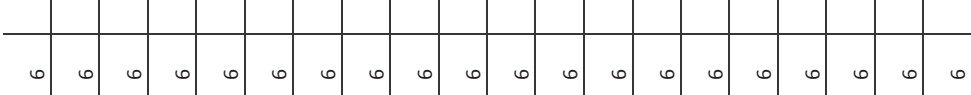

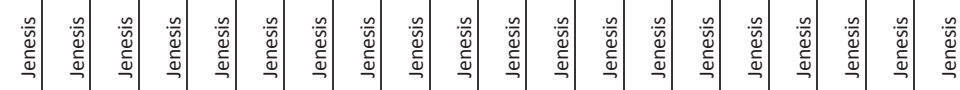




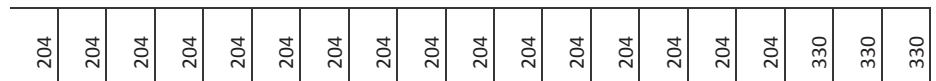

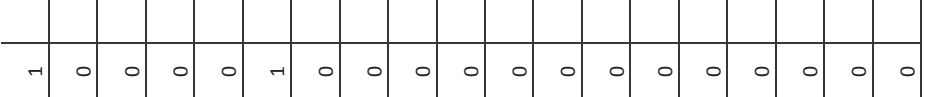

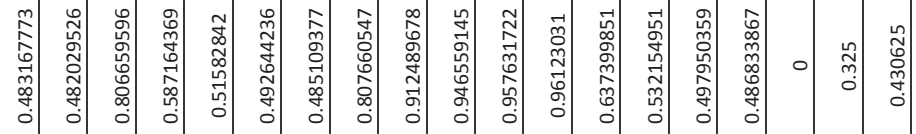

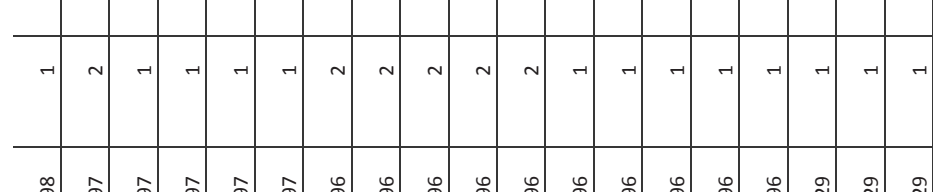

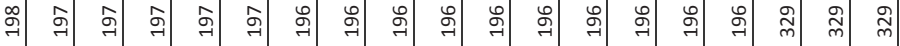

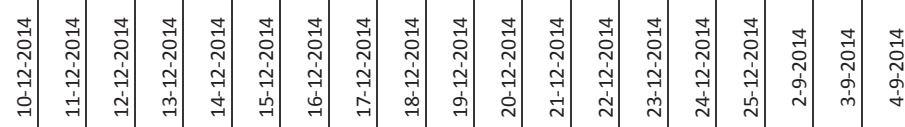

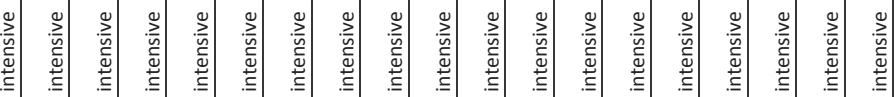
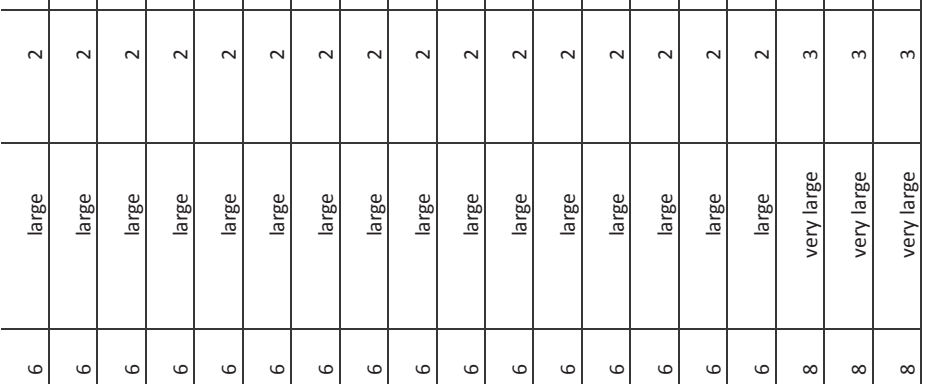

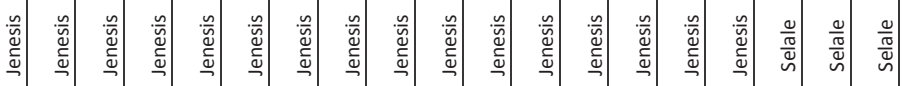




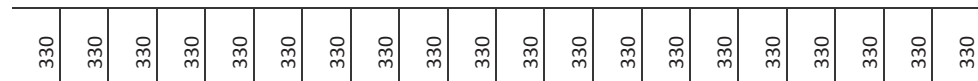

\begin{tabular}{lllllllllllllllllllll}
\hline 0 & 0 & 0 & 0 & 0 & 0 & 0 & 0 & 0 & 0 & 0 & 0 & 0 & 0 & 0 & 0 & 0 & 0 & 0 & 4
\end{tabular}

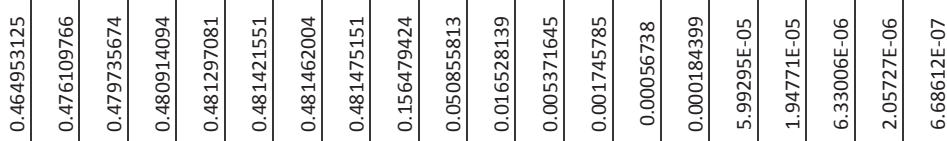

\begin{tabular}{|c|c|c|c|c|c|c|c|c|c|c|c|c|c|c|c|c|c|c|c|}
\hline & - & & & & & & & & & & & & & & & & & & \\
\hline & $\neg$ & $\neg$ & -1 & 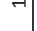 & $\overrightarrow{7}$ & $\vec{\gamma}$ & ${ }^{\circ}$ & 0 & 0 & 0 & 0 & 0 & 0 & 0 & 0 & 0 & 0 & o & \\
\hline శ్లి & శ్లి & శ్లి & జ్లి| & స్లి| & జ్లి & స్లి & స్లి & స్లి & స్లి & శ్లి & $\underset{్}{\text { ని }}$ & స్లి & స్లి| & స్లి & శ్లి & స్ & శ్లి| & శ్లి| & \\
\hline
\end{tabular}

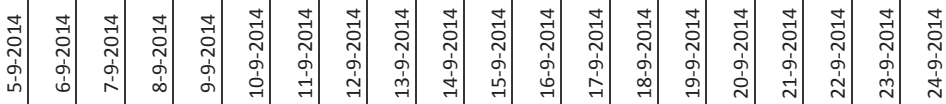

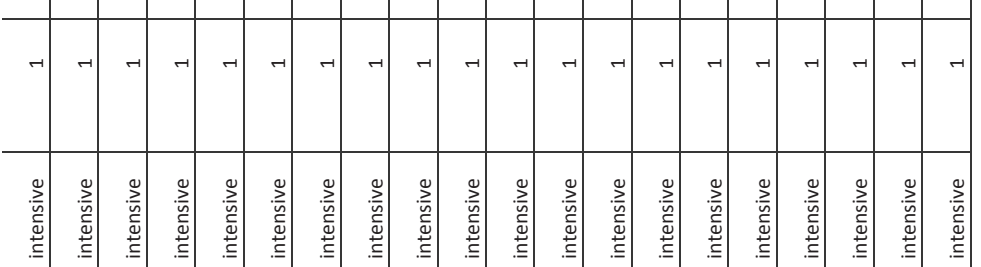

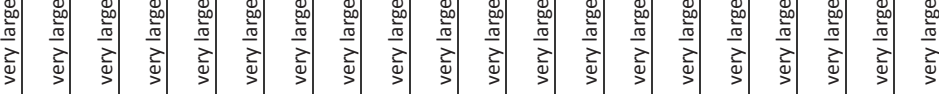

\begin{tabular}{llllllllllllllll|l|l|l|l|l}
\hline & $\infty$ & $\infty$ & $\infty$ & $\infty$ & $\infty$ & $\infty$ & $\infty$ & $\infty$ & $\infty$ & $\infty$ & $\infty$ & $\infty$ & $\infty$ & $\infty$ & $\infty$ & $\infty$ & $\infty$ & $\infty$ & $\infty$
\end{tabular}

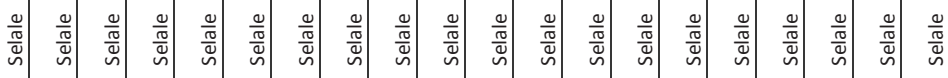


శ్లి శ్లి శ్లి

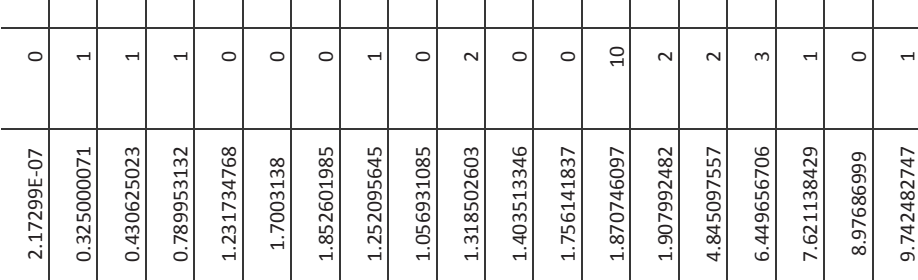

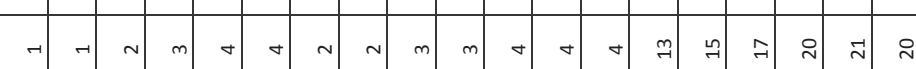

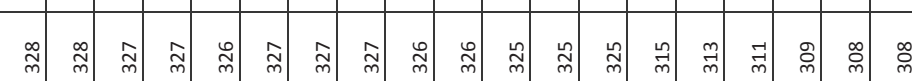

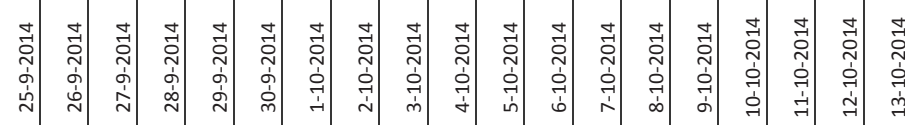

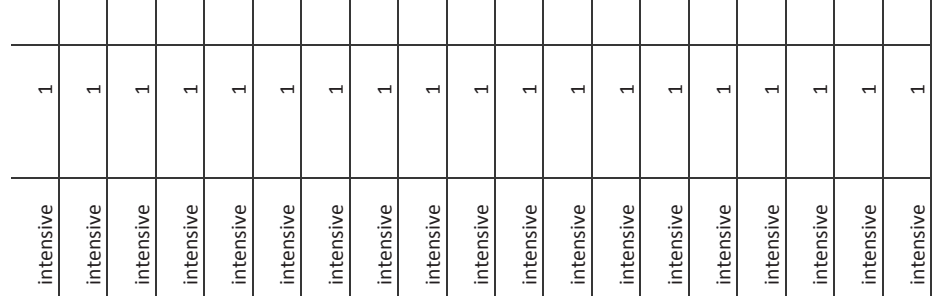

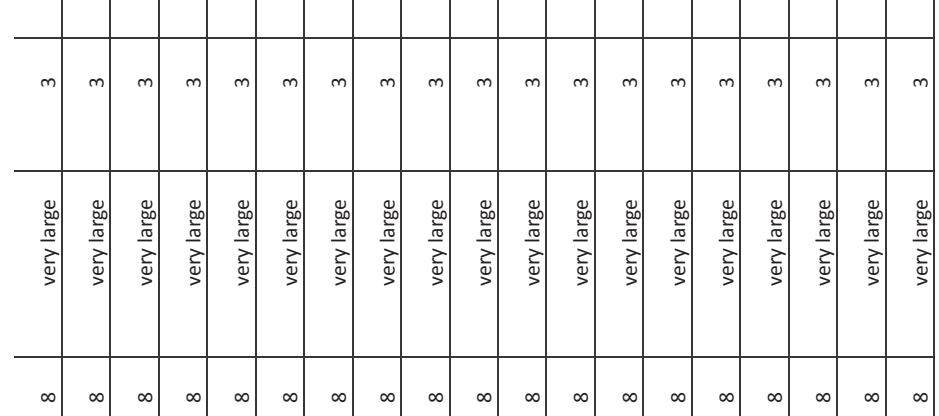

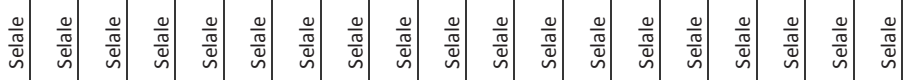




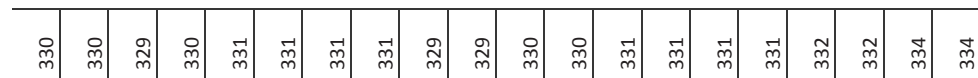

\begin{tabular}{l|lll|l|l|l|l|l|l|l|l|l|l|l|l|l|l|l|l|l|l}
\hline 0 & 4 & 0 & 4 & 0 & $m$ & 4 & 0 & 0 & 0 & 4 & 4 & 0 & 0 & 4 & 4 & 4 & $n$ & 0 & $n$ \\
\hline
\end{tabular}

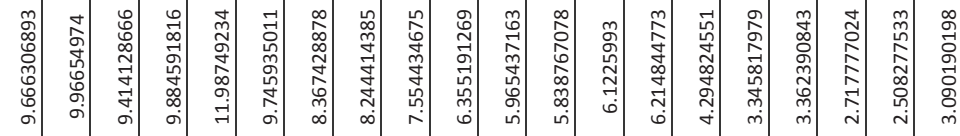

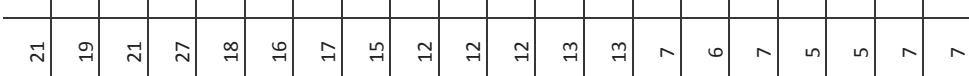

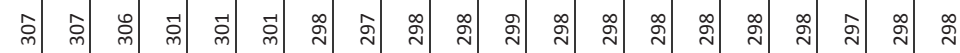

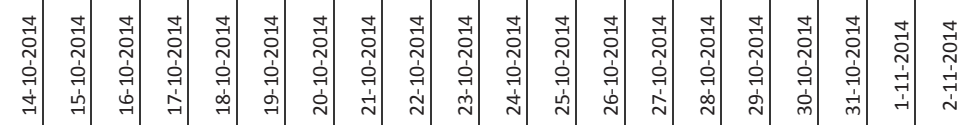

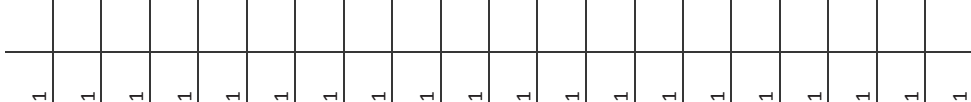

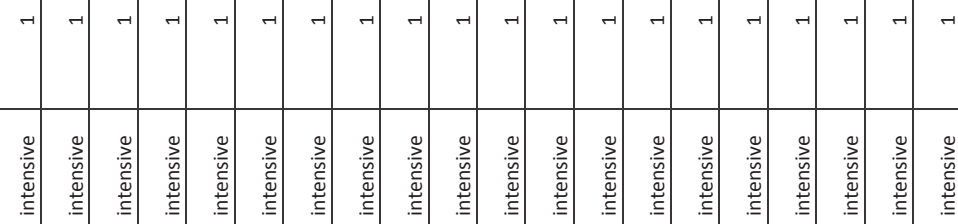

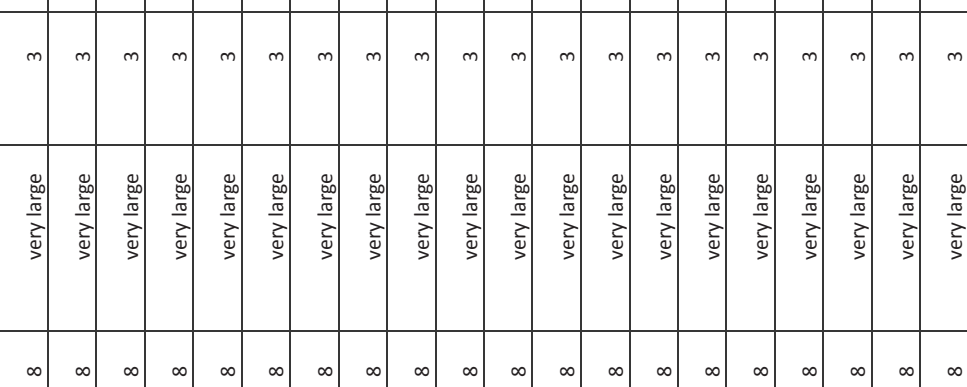

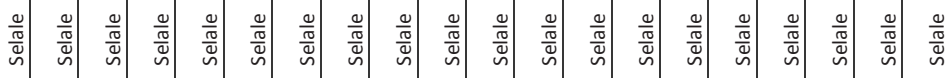


弚

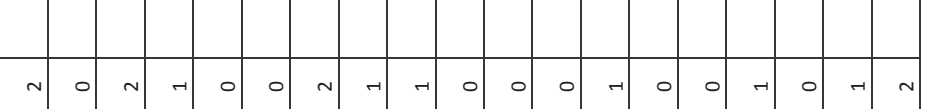

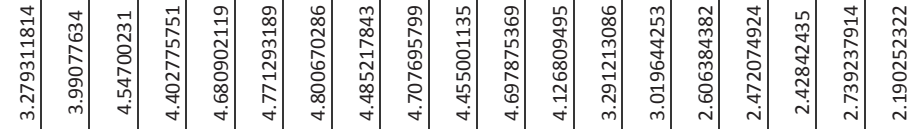

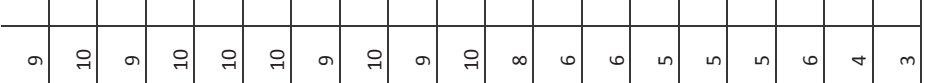

品 品

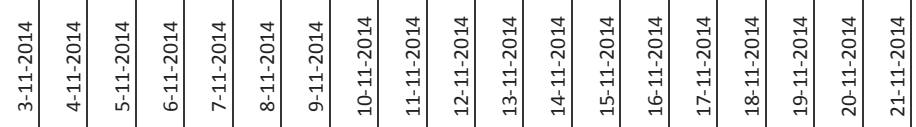

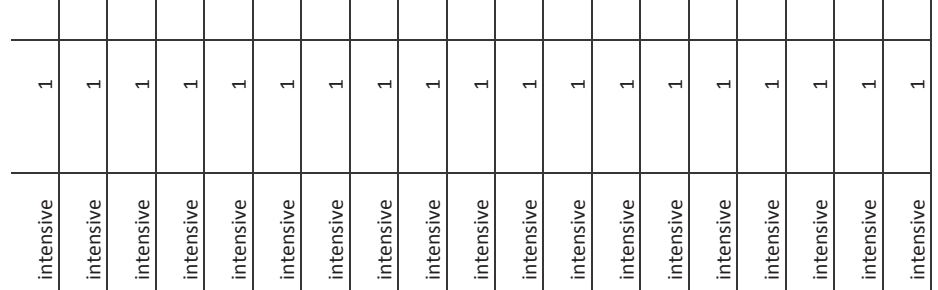

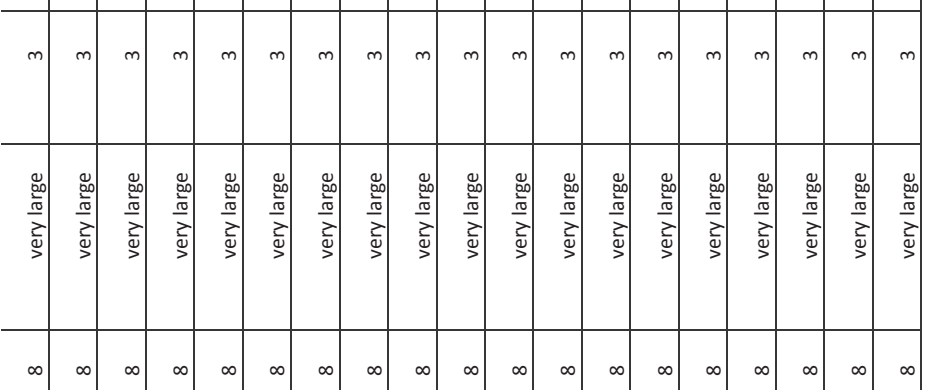

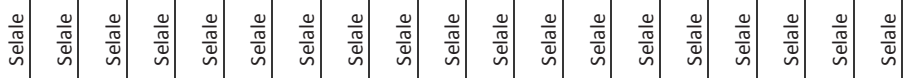




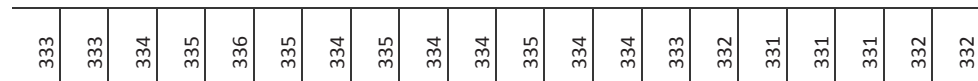

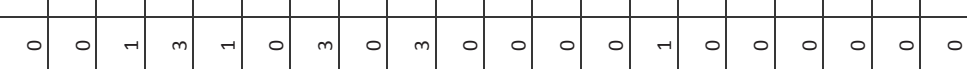

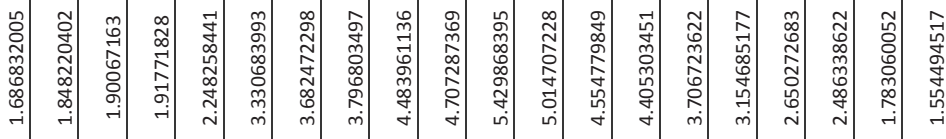

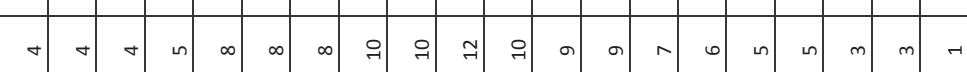

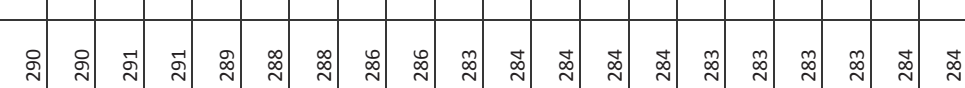

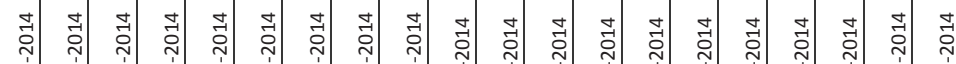

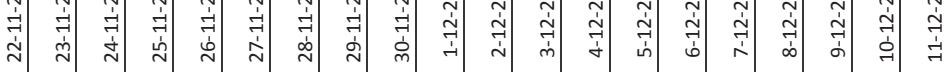

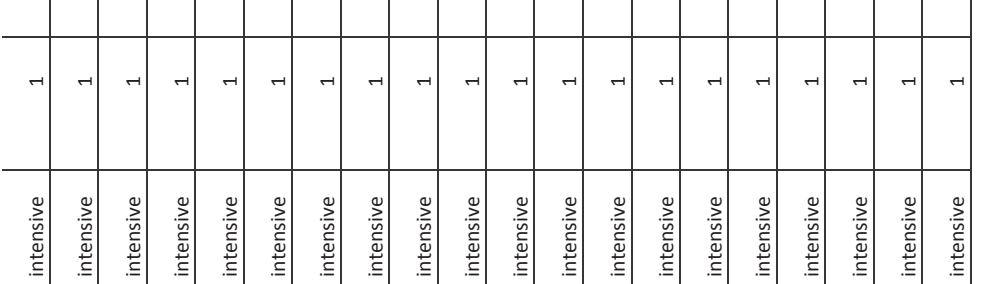

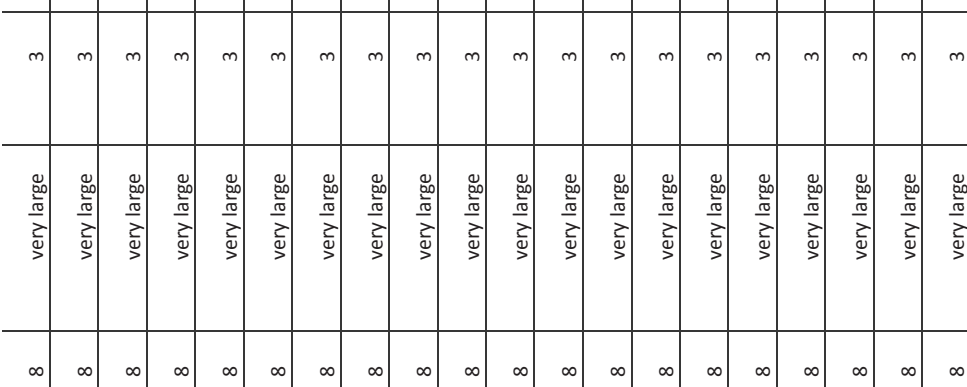

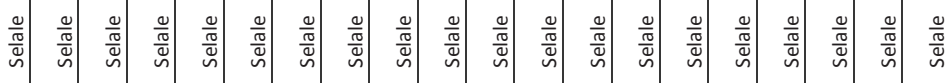




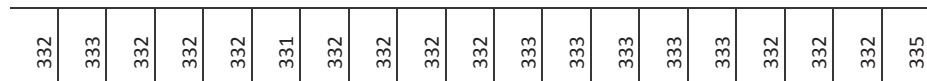

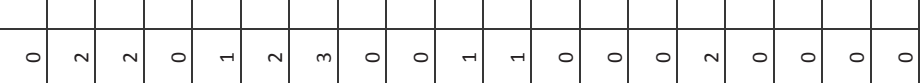

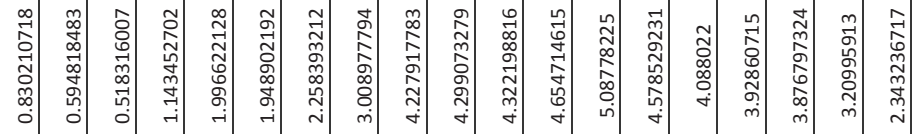

\begin{tabular}{|c|c|c|c|c|c|c|c|c|c|c|c|c|c|c|c|c|c|c|}
\hline-1 & $\rightarrow$ & & & & & & & & & & & & & & & & & \\
\hline & & & & 8 & n) & 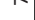 & $\rightarrow$ & 0 & 0, & $\rightarrow 1$ & 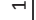 & का & $\infty$ & $\infty$ & $\infty$ & 8 & $\sigma$ & $\nabla$ \\
\hline$\underset{\sim}{\mathbb{\infty}}$ & $\stackrel{\sim}{\infty}$ & $\underset{\sim}{\sim}$ & $\underset{\sim}{\sim}$ & 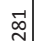 & 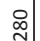 & 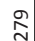 & $\stackrel{0}{\stackrel{2}{v}}$ & $\stackrel{\wedge}{\Sigma}$ & న & $\hat{\imath}$ & ڤొ & $\begin{array}{l}0 \\
2\end{array}$ & $\stackrel{0}{2}$ & ○ & ț & $\stackrel{t}{\Delta}$ & $\stackrel{+}{\Delta}$ & $\widehat{N}$ \\
\hline
\end{tabular}

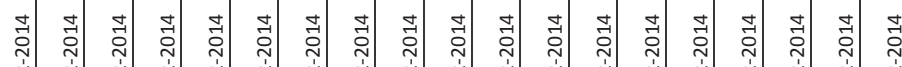

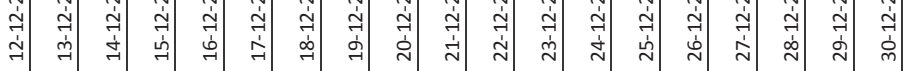
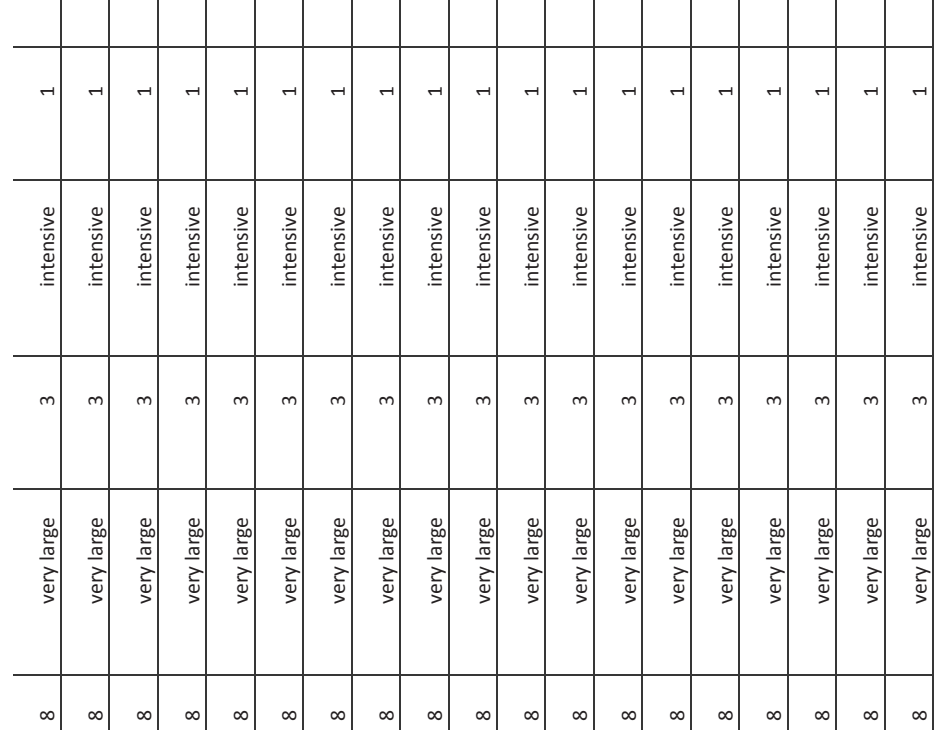

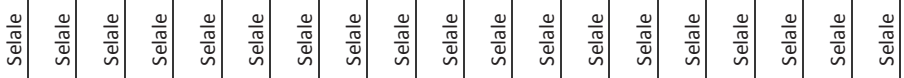




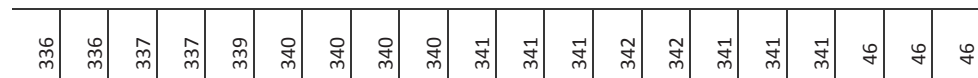

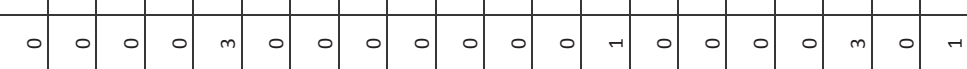

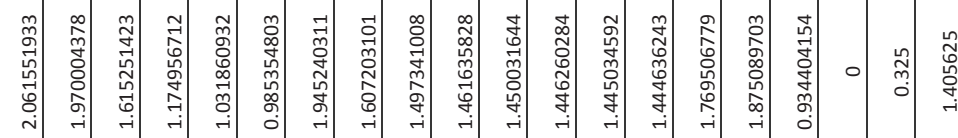

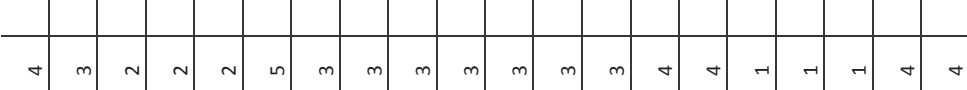

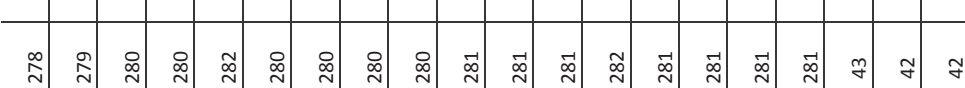

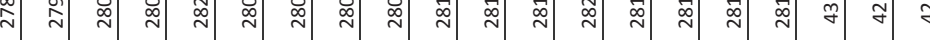

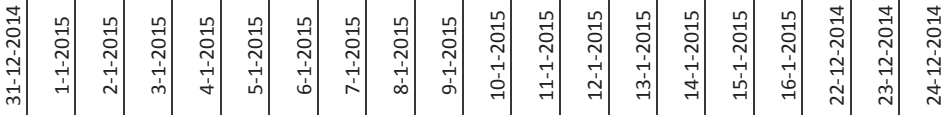

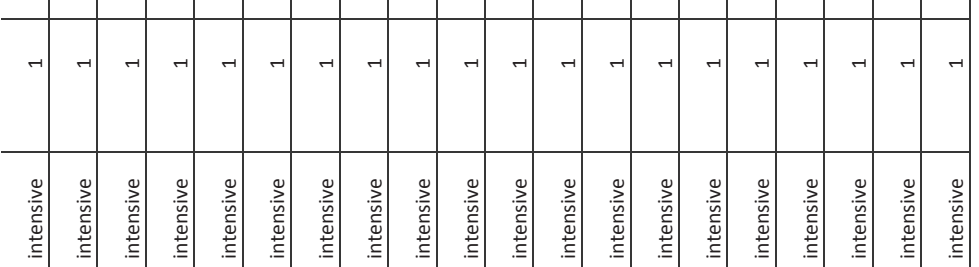

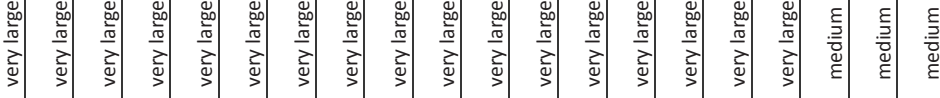

\begin{tabular}{llllllllllllllllllllllll}
\hline & $\infty$ & $\infty$ & $\infty$ & $\infty$ & $\infty$ & $\infty$ & $\infty$ & $\infty$ & $\infty$ & $\infty$ & $\infty$ & $\infty$ & $\infty$ & $\infty$ & $\infty$ & $\infty$ & $\sigma$ & $\sigma$ & $\sigma$
\end{tabular}

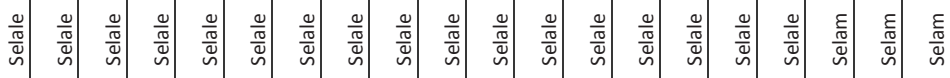



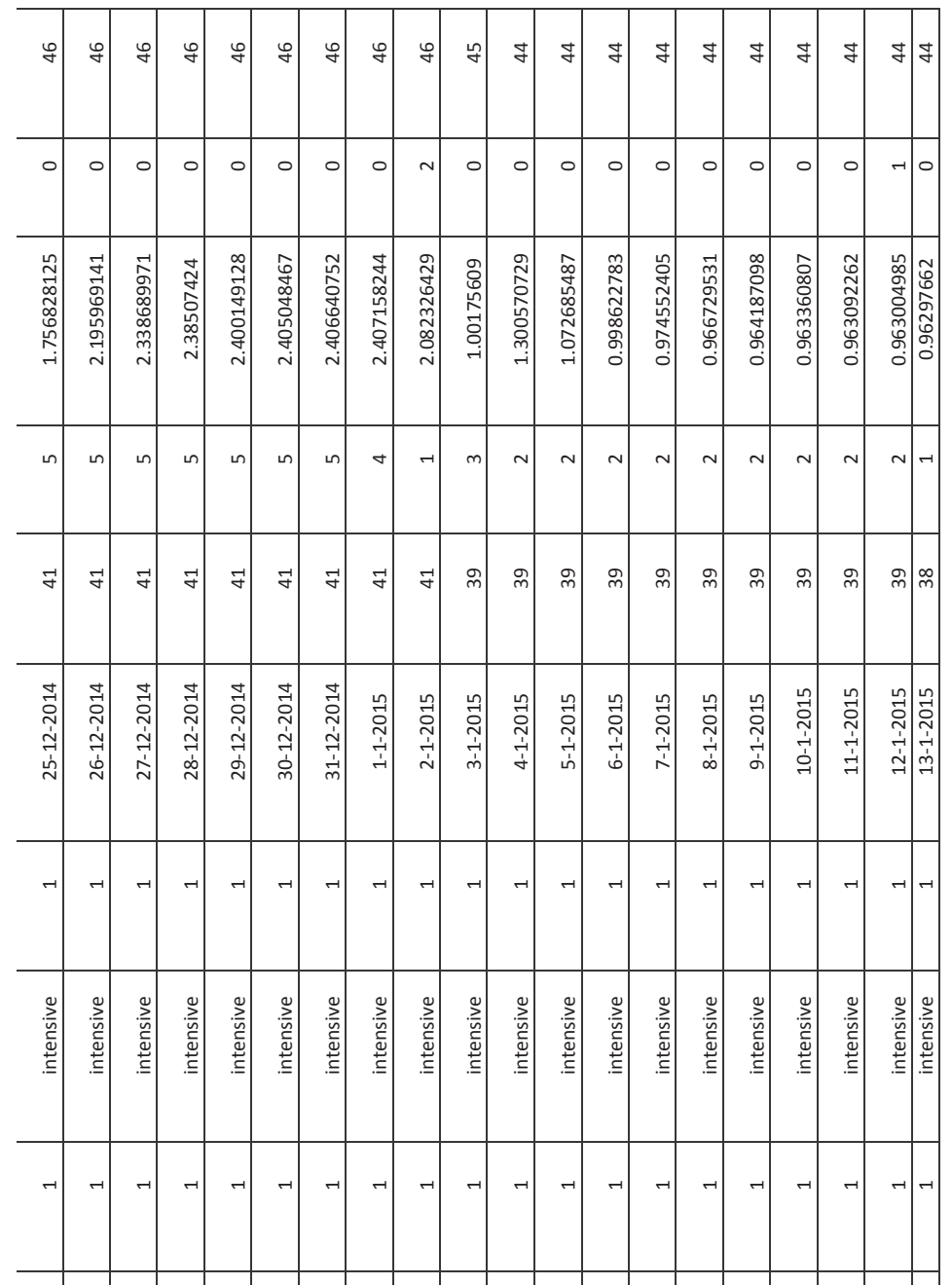

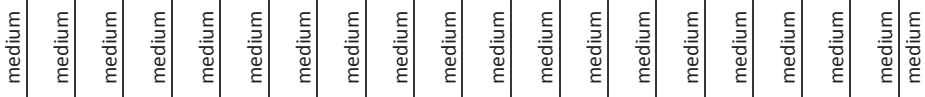

a

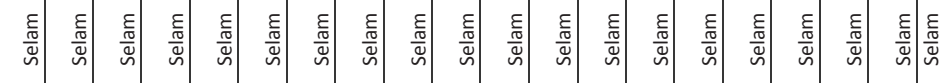




\begin{tabular}{|c|c|c|c|c|c|c|c|c|c|c|c|c|c|c|c|c|c|c|}
\hline 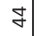 & 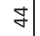 & 寸 & F & 寸 & F & 寸 & g & J & J & J & J & J & $\forall$ & $\forall$ & J & J & Z & J \\
\hline 0 & 0 & 0 & 0 & 0 & 0 & 0 & 0 & $\rightarrow$ & 0 & 0 & 0 & 0 & 0 & 0 & 0 & 0 & 0 & ○ \\
\hline 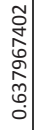 & 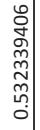 & $\begin{array}{l}\hat{0} \\
\tilde{o} \\
0 \\
0 \\
0 \\
o \\
0 \\
0\end{array}$ & $\begin{array}{l}n \\
0 \\
0 \\
0 \\
0 \\
0 \\
0 \\
0 \\
0\end{array}$ & 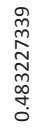 & 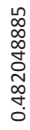 & $\begin{array}{l}\infty \\
0 \\
0 \\
0 \\
0 \\
6 \\
0 \\
0 \\
0 \\
0\end{array}$ & $\begin{array}{l}m \\
\stackrel{m}{7} \\
\vec{v} \\
5 \\
0 \\
0 \\
0 \\
0\end{array}$ & 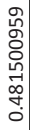 & 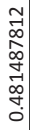 & 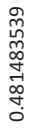 & 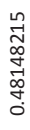 & 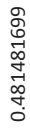 & 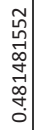 & $\begin{array}{c}0 \\
0 \\
h \\
0 \\
0 \\
0 \\
0 \\
+ \\
0\end{array}$ & 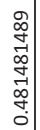 & 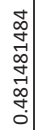 & 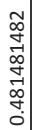 & 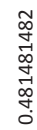 \\
\hline-1 & $\rightarrow$ & -1 & -1 & -1 & $\rightarrow$ & -1 & -1 & $\neg$ & -1 & -1 & -1 & -1 & -1 & -1 & -1 & -1 & -1 & 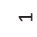 \\
\hline$\stackrel{\infty}{m}$ & $\stackrel{\infty}{m}$ & $\stackrel{\infty}{m}$ & $\stackrel{\infty}{m}$ & $\stackrel{\infty}{m}$ & $\stackrel{\infty}{m}$ & $\stackrel{\infty}{m}$ & $\stackrel{\infty}{m}$ & $\stackrel{\infty}{m}$ & $\hat{m}$ & $\hat{m}$ & $\hat{m}$ & $\hat{m}$ & $\hat{m}$ & $\hat{m}$ & $\hat{m}$ & $\hat{m}$ & $\hat{m}$ & $\widehat{m}$ \\
\hline & & & & & & ᄂ & & & & & & & & & & & & \\
\hline రి & ָัర & ָे & ָे & ָָ & ָे & ્ָণ & ્ָગ & ర్స & స్ & i্ & 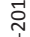 & 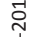 & స్ర & స్ర & స్ర & స్ర & స్ర & D্ \\
\hline$\vec{d}$ & 岁 & $\vec{b}$ & $\overrightarrow{1}$ & 崩 & هু & 仓் & $\vec{\Delta}$ & $\overrightarrow{\tilde{N}}$ & $\overrightarrow{\tilde{n}}$ & $\stackrel{\vec{d}}{N}$ & ஸे & $\overrightarrow{0}$ & $\vec{\sim}$ & 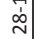 & $\overrightarrow{\grave{d}}$ & $\vec{\rho}$ & $\vec{m}$ & \\
\hline
\end{tabular}

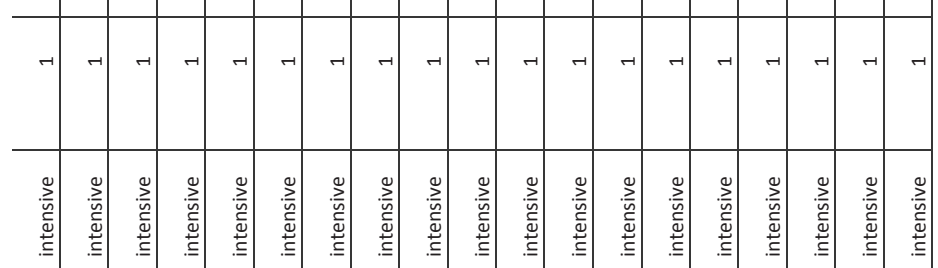

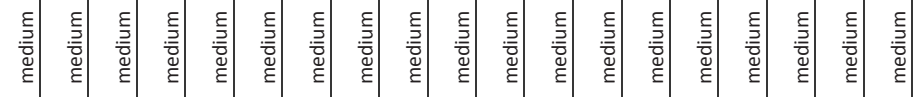

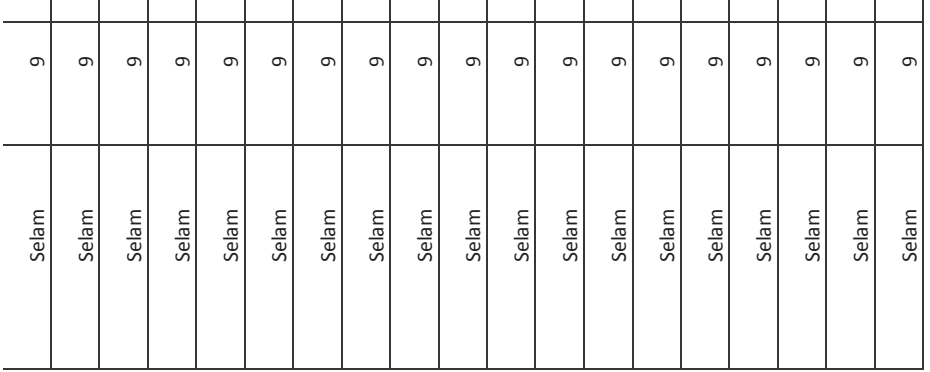




\section{Field study on the use of vaccination to control the occurrence of lumpy skin disease in Ethiopian cattle}

Wassie Molla ${ }^{a, b}$, Klaas Frankena ${ }^{a}$, Getachew Garic, Mart C.M. de Jong ${ }^{a}$

a Quantitative Veterinary Epidemiology, Wageningen University \& Research, Droevendaalsesteeg 1, 6708 PB Wageningen, The Netherlands

${ }^{b}$ Faculty of Veterinary Medicine, University of Gondar, P.O. Box 196, Gondar, Ethiopia

${ }^{\mathrm{c}}$ National Animal Health Diagnostic and Investigation Centre (NAHDIC), Sebeta, Ethiopia

Preventive Veterinary Medicine (2017) 147: 34-41

DOI: http://dx.doi.org/10.1016/j.prevetmed.2017.08.019. 


\begin{abstract}
The current study was carried out in central and north-western parts of Ethiopia to assess the efficacy of Kenyan sheep pox virus strain vaccine (KS1 0-180) against natural lumpy skin disease (LSD) infection under field conditions by estimating its effect on the transmission and severity of the disease. For this study, an LSD outbreak was defined as the occurrence of at least one LSD case in a specified geographical area. An observational study was conducted on a total of 2053 (1304 vaccinated and 749 unvaccinated) cattle in 339 infected herds located in 10 subkebeles and a questionnaire survey was administered to 224 herd owners. Over $60 \%$ of the herd owners reported that the vaccine has a low to very low effect in protecting animals against clinical LSD; almost all of them indicated that the vaccine did not induce any adverse reactions. In the unvaccinated group of animals $31.1 \%$ were diagnosed with LSD while this was $22.5 \%$ in the vaccinated group $(P<0.001)$. Severity of the disease was significantly reduced in vaccinated compared to unvaccinated animals ( $\mathrm{OR}=0.68,95 \% \mathrm{Cl}: 0.49 ; 0.96)$. Unvaccinated infected animals were more likely (predicted fraction $=0.89$ ) to develop moderate and severe disease than vaccinated infected animals (predicted fraction $=0.84$ ).

LSD vaccine efficacy for susceptibility was estimated to be 0.46 (i.e. a susceptibility effect of 0.54 ) while the infectiousness effect of the vaccine was 1.83 . In other words, the vaccine reduces the susceptibility by a factor of two and increases infectiousness by approximately the same amount. LSD transmission occurred in both vaccinated and unvaccinated animals, the estimated reproduction ratio (R) was 1.21 in unvaccinated animals compared to 1.19 in vaccinated ones, and not significantly different. In conclusion, KS1 0-180 vaccination, as applied currently in Ethiopia, has poor efficacy in protecting cattle populations against LSD, neither by direct clinical protection nor by reducing transmission, and this signifies the urgent need to either improve the quality of the vaccine or to develop potent alternative vaccines that will confer good protection against LSD.
\end{abstract}

Key words: Capripoxvirus, Kenyan sheep pox (KS1 O-180) vaccine, Lumpy skin disease, Reproduction ratio, Severity, Vaccine efficacy 


\subsection{Introduction}

Lumpy skin disease (LSD) is a disease of cattle caused by LSD virus, a DNA virus, which belongs to the family Poxviridea, subfamily Chordopoxvirinae and it is of the genus Capripoxvirus. The disease is characterized by fever, nodular lesions on the skin and mucous membranes, inflammatory and oedematous swellings of the limbs and brisket, lymphadenopathy, deterioration of body condition and drop in milk production (Davies, 1991; Quinn et al., 2002; Radostits et al., 2007). It has spread to most African countries, Middle East countries and recently to Europe (Davies, 1991; Tuppurainen and Oura, 2012; Tasioudi et al., 2016; WAHID, 2016; Tuppurainen et al., 2017). LSD is endemic in Ethiopia and is a constant threat to the livestock sector since its first occurrence in 1981 (Mebratu et al., 1984; Gari et al., 2010). LSD outbreaks occur frequently in various regional states of the country, despite intensive vaccination campaigns (APHRD, 2012). It is an economically devastating and therefore a notifiable disease as per OIE disease categorization (Gari et al., 2011; Tuppurainen and Oura, 2012; OIE, 2016).

Vaccination, movement control and slaughter of infected and in-contact animals are considered as options for the control of LSD. However, it is widely agreed that vaccination is the most manageable and realistic approach to control the disease in endemic and resource poor countries (Carn, 1993; Tuppurainen et al., 2014). Live attenuated vaccines based on sheep pox virus (for example, Kenyan sheep pox (KS1 0-180), Romanian sheep pox and Yugoslavian RM 65 sheep pox vaccines), goat pox virus (Gorgan goat pox vaccine), and LSDV (Neethling strain vaccine) have been used for the control of LSD (OIE, 2010; Tuppurainen et al., 2014; Gari et al., 2015).

In general vaccination can exert important effects, both at the individual and at the population level. It may help to directly protect vaccinated animals, reduce severity of the disease by reducing all or some of its symptoms or it may reduce transmission of pathogens by lowering susceptibility and/or infectiousness, and thus also indirectly reduce the risk for other vaccinated and unvaccinated individuals to become infected (De Jong, 1994; Halloran et al., 1997; Van der Goot et al., 2007; Aznar et al., 2011; OIE, 2015). The effect of vaccine intervention on the dynamics of infectious diseases, i.e. in the population, can be estimated by the reproduction ratio $(R)$ which is the average number of secondary cases arising from one typically infected animal during its entire infectious period (Diekmann et al., 1990; Heffernan et al., 2005).

LSD vaccine failure has been reported in several countries including Ethiopia. During the 2006 outbreak in Israel, 11\% (4.2\% in dairy and $33.7 \%$ in feedlot 
cattle) of RM65 (Ramayer strain) vaccinated cattle became infected (Brenner et al., 2009). In Jordan, Abutarbush (2014) reported an overall LSD morbidity of $4.7 \%$ in cattle populations vaccinated with RM65 (Jovivac ${ }^{\circledR}$ ) and LSD vaccine of unknown origin. Kumar (2011) reported a continued LSD outbreak in Oman for more than three months after vaccination of cattle herds with Kenyan sheep and goat pox vaccine. In Ethiopia, LSD vaccine failure has been reported since 1993 (Carn, 1993). Ayelet et al. (2013) estimated morbidity to be $23.8 \%$ in the cattle population of central Ethiopia after vaccination with KS1 0-180 virus strain vaccine. However, a better protection was claimed with Neethling vaccine (1.11\% morbidity) and with a 10 times higher dose of the RM65 vaccine (1.85\% morbidity) (Ben-Gera et al., 2015). Vaccines in general may give only partial protection (leaky vaccines) or protect only some of the individuals (all-or-nothing) (Smith et al., 1984). In addition, further immunization failure may arise due to insufficient vaccine coverage or factors related to the host, vaccine, or vaccination quality due to handling, reconstitution or administration of the vaccine (Quinn et al., 1999).

Ayelet et al. (2013) and Gari et al. (2015) reported that KS1 O-180 vaccine provides incomplete protection in immunized animals. However, the level of protection and its effect on the severity of the disease have not been documented well under field conditions. KS1 0-180 vaccine is still applied as the sole means of LSD control in Ethiopia. Hence, the aims of this study was to assess the efficacy of KS1 0-180 virus strain vaccine against natural LSD infections under field conditions by estimating its effect on the transmission and severity of the disease.

\subsection{Materials and Methods}

\subsubsection{Study and study area}

The study consisted of two parts:

(1) A questionnaire survey focusing on herd owners' information regarding several aspects of vaccination which was undertaken in central and north-western parts of Ethiopia (Figure 5.1). In central Ethiopia, it was undertaken in Ada'a, Sebeta Hawas, Ambo, Dendi, Debrelibanos, Kuyu and Hidabu Abote districts in Oromia National Regional State. In north-western part, the data were collected from Dejen, Gozamen, Hulet Ejju Enessie and Jabitenan districts in Amhara National Regional State. The dominant agricultural production system in the study areas was mixed crop-livestock system. The grazing practice in almost all study areas was open grazing on communal pasture land where animals from a village were herded together. 
(2) A vaccine efficacy follow-up study under field conditions was undertaken in the north-western part of Ethiopia in Mota town and the surrounding four rural kebeles (the lowest administrative structure in Ethiopia, in which at least 500 households (3,500 to 4,000 persons) live and cover on average about $53 \mathrm{~km}^{2}$ and $3 \mathrm{~km}^{2}$ land area in rural and urban places, respectively) of Hulet Ejju Enessie district, East Gojjam Administrative Zone, Amhara National Regional State (Figure 5.2). The rural kebeles were Hibre Selam, Debre Gubae, Beza Bizuhan, and Ayen Berhan. Cattle populations of ten sub-kebeles were enrolled in the study namely Mota (from Mota town), Akobe, Semo, and Shewaber from Hibre Selam, Atetanat and Yerez from Beza Bizuhan, Webmariam from Ayen Berhan, and Kesmender, Komma and Zenabach from Debre Gubae kebele.

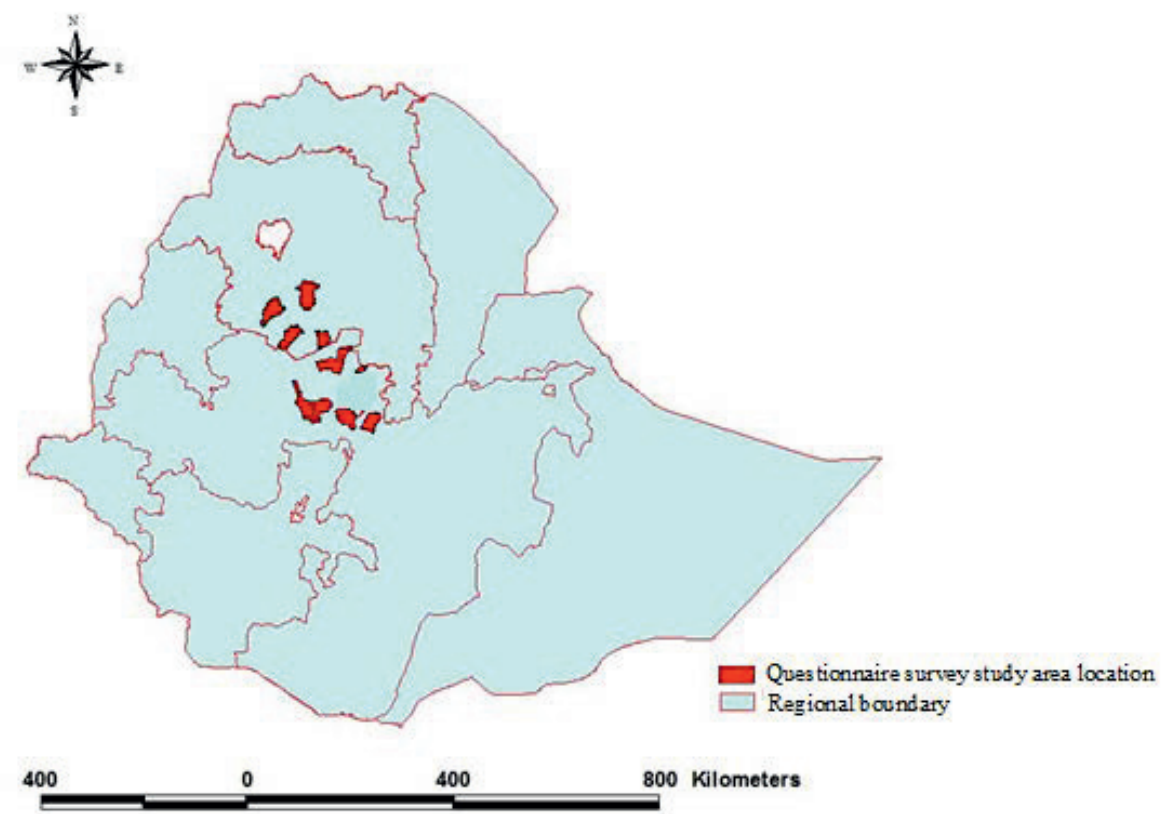

Figure 5.1 Map of Ethiopia showing the area where the questionnaire survey was performed. 


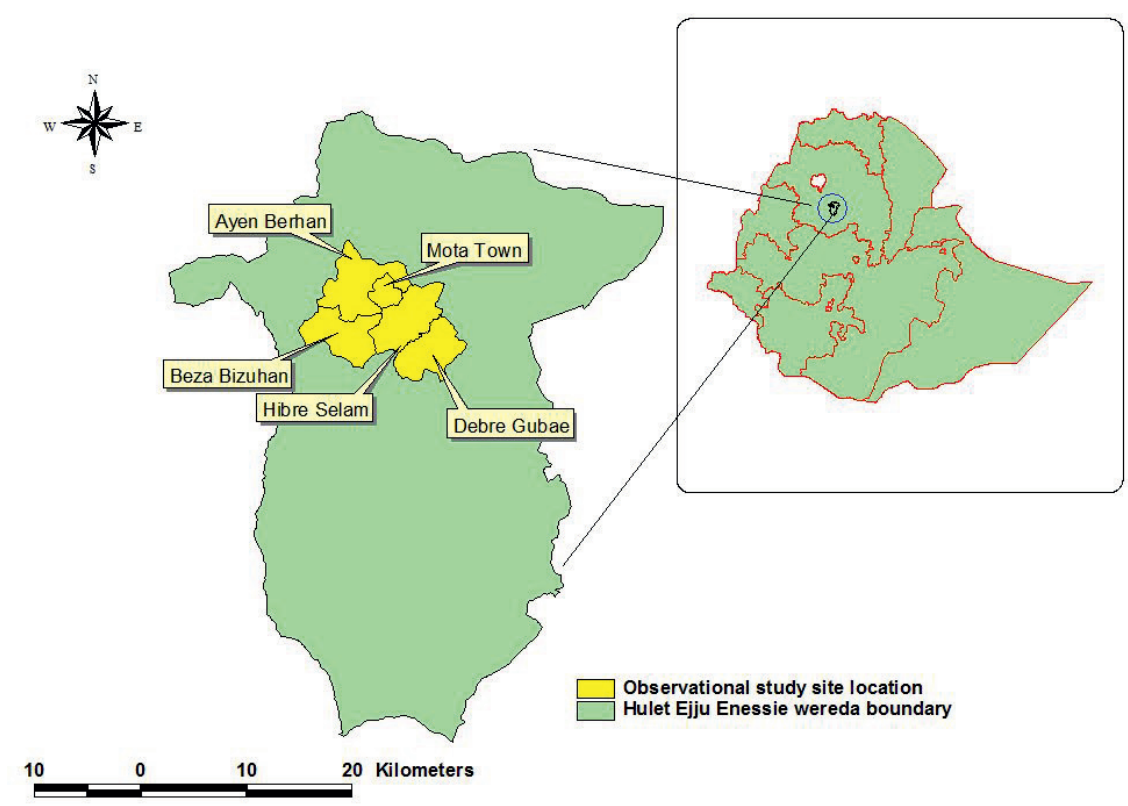

Figure 5.2 Map of Hulet Ejju Enessie district (Ethiopia) showing LSD vaccine efficacy observational study site.

\subsubsection{Questionnaire survey}

The study population for the questionnaire survey was about 13,200 cattle herd owners living in 33 selected kebeles of 11 districts. These owners were smallholder farmers with the main purpose of subsistence farming, that is: draft power for crop production, milk for consumption, manure for soil fertility and fuel, and cash income. Animals were kept in an extensive management system and most of the herds were composed of local Zebu breed cattle. Animals in this system share communal grazing and watering resources. The term "herd" in this study designates an aggregate of animals kept together day and night and owned by a household.

\subsubsection{Study design and data collection}

Eleven districts located in the central and north-western parts of Ethiopia were identified for a cross-sectional questionnaire survey. The districts were selected based on the recent LSD outbreak occurrence, location and accessibility. For this study, an LSD outbreak was considered, if at least one case of LSD occurred in a specified geographical area (usually kebele). Three kebeles were randomly selected 
from each district. From each kebele, five to eight herd owners willing to participate were interviewed. The survey data were collected from a total of 224 herd owners from January 2015 to May 2015.

The data were collected by face to face interview using the local language. After getting an informed consent from the herd owners, the interviewer asked questions about the vaccination status, vaccination frequency, the vaccination service provider, fee of the vaccination, the vaccination date and when the animals become infected if there was any infected animal in his herd. Furthermore, the herd owners were requested to express their opinion on the effectiveness of the vaccine in protecting cattle against LSD and the adverse reactions to the vaccine. The vaccine is considered to be protective from day 21 to one year post vaccination. All responses were recorded in a predesigned response sheet.

\subsubsection{Data management and analysis}

Descriptive statistics were used to summarise the data on vaccination coverage at herd level, frequency of vaccination, and owner's opinion about the effectiveness and adverse reactions of the LSD vaccine.

\subsubsection{Follow-up study}

A follow-up study was carried out after the index case of LSD appeared in Beza Bizuhan kebele at the specific village called Chech on 29 April 2014. The disease stayed restricted in the village for a reasonable period of time but after that it spread to other villages and surrounding kebeles. The selected area for follow-up was Mota town and its surrounding area, representing 10 sub-kebeles. In the area, animals were owned by smallholder farmers with the main purpose of subsistence farming except for six dairy farms which kept cattle for commercial purposes. Most of the herds were composed of local Zebu breed cattle and managed under extensive management. The six dairy herds consisted mainly of Holstein-Zebu cross and were managed under semi-intensive or intensive conditions.

The study population included 7464 heads of cattle grouped in 1203 herds. The cattle population in each sub-kebele (considered as ten separate populations as they were herded on common pasture land within a sub-kebele) was vaccinated partially. This partial coverage was not purposive but due to the failure of the owner to get their animals vaccinated. The vaccination campaign was undertaken at least one month before the entrance of the disease into a specific sub-kebele. The 
vaccination was provided by the public veterinary service of the Hulet Ejju Enessie district following the index case appearance in the area.

\subsubsection{Study design and herd selection}

This study was designed as a prospective cohort study. At the beginning of the study, ten cattle populations (i.e. all cattle in a sub-kebele excluding calves less than 6 month old) with partial vaccination coverage and LSD free status were selected. All herds in the selected populations were inspected on a weekly basis for clinical signs of LSD. The herd owners were also asked to report any suspicion of the disease. The sub-kebeles were selected based on their partial vaccination status. We selected populations with different vaccination coverage because that is a prerequisite to estimate both vaccine efficacy for susceptibility and infectiousness (Longini et al., 1998; Aznar et al., 2011). The vaccination coverage level in the selected 10 sub-kebele cattle populations ranged from 3-95\%. Since the vaccine coverage was strictly inferior to $100 \%$, a number of infections within the vaccinated group was expected to occur. The animals, whether vaccinated or not, were followed starting from August 1, 2014 to November 31, 2014, i.e. from the day the first case was detected in the sub-kebele until no more new cases were recorded. If an animal in a herd was diagnosed with LSD and the owner volunteered to participate, the herd was enrolled in the study. Therefore, the main inclusion criteria for a herd were the infection status of the herd and the willingness of the owner to participate. A herd was considered positive if at least one animal showed LSD-characteristic nodular lesions. In total, 448 herds were recorded as being affected and of these, 339 farmers (75.7\%) were willing to participate and all their bovines $(n=2053)$ enrolled in the study.

\subsubsection{Data collection}

In the ten sub-kebeles, infected herds were visited twice a week by animal health professionals and by the first author, and clinical signs were recorded. The severity of LSD was assessed at three levels: mild, moderate and severe. Mild LSD was defined as only few nodular lesions $(<5)$ in some part of the body, mild fever (39$39.5^{\circ} \mathrm{C}$ ) and quick recovery (within a week); the moderate level was assigned if fever, inappetence, many nodular lesions/swelling on the limb or brisket, and weakness was present; severe LSD was scored if high fever $\left(>40^{\circ} \mathrm{C}\right)$, extensive nodular lesions/swellings, anorexia, weakness, emaciation or death was observed.

Animal data including breed, sex, age and records like vaccination status, vaccination date and type of vaccine used were compiled for all animals at the first 
herd visit. Type of herd and sub-kebele were also recorded. The first visit was made by the district veterinary team and the first author. The animal health professionals who collected the data from infected animals were blind for the vaccination status of the affected animals.

Biopsy samples of skin nodules were collected from a sample of the affected animals in each sub-kebele and analysed by conventional and Snapback Real-time PCR (polymerase chain reaction) techniques following the method described by Tuppurainen et al. (2005) and Gelaye et al. (2013) to confirm that the clinically observed disease truly was LSD. A total of 34 skin samples were collected for LSD confirmation.

\subsubsection{Vaccine used for control and prevention of LSD}

The live attenuated vaccine of KS1-0180 produced by National Veterinary Institute (NVI), Ethiopia was the only vaccine used for prevention and control of LSD in Ethiopia. It recently has been reported that the virus used for the production of KS1-O180 is not a sheep pox virus but was found to be LSDV (Gelaye et al., 2015). The vaccine was prepared in $20 \mathrm{ml}$ vials containing 100 doses and reconstituted by $100 \mathrm{ml}$ of cool and sterile saline water; $10^{3.5} \mathrm{TCID}_{50}$ was administered per animal as recommended by the manufacturer. A suspension of $1 \mathrm{ml}$ vaccine was injected subcutaneously at the neck side (NVI, 2010 ).

\subsubsection{Data management and analysis}

Descriptive statistics were used to describe the morbidity and mortality in cattle populations with different vaccination coverage.

To analyse the association between the occurrence of LSD infections in animals (i.e. the cases, which are assumed to be binomial distributed) and independent variables (vaccination status, breed, age, sex, herdtype, and location), multivariable logistic regression was performed (STATA version 14). Vaccination status was the main effect of interest while location, breed, age, sex and herdtype were added as additional explanatory variables. All factors were fitted in a multivariable regression model and the final model was obtained by a backward stepwise elimination procedure while checking for confounding. For that purpose confounding was defined as a change of at least $25 \%$ in any of the regression coefficients after removing a non-significant $(p>0.05)$ variable from the model. Interactions were tested for all combinations of the significant main effects. Generalised estimating equations (GEE, population averaged model) was run using herd as random effect. An exchangeable correlation structure was specified for the 
random effect and results were expressed as Odds ratio (OR) and its $95 \%$ confidence interval $(\mathrm{Cl})$.

To estimate the effect of vaccination on the severity (mild, moderate or severe) of LSD, first a univariable and then multivariable (backwards elimination process) ordered logistic regression analysis was run by incorporating breed, age, sex, herdtype, and kebele as potential factor and retaining it in the model as confounder when necessary. The probability of a vaccinated or unvaccinated infected animal falling in either of the severity categories was computed using estimated coefficients and the associated cut points of the ordered logistic regression analysis. Proportionality of odds across response categories was tested using the approximate likelihood-ratio test (omodel logit command in STATA version 14).

Multivariable regression analysis using a generalized linear model (GLM) was performed to assess the effect of vaccination on the transmission of LSD by setting LSD infection of animals as binomial (yes/no) dependent variable and vaccination status (yes/no) and fraction of vaccinated among the infected (FracVaccl) as independent variables. The model was fit using the complementary loglog (cloglog) link function and log (number of infected animals/total number of animals per sub-kebele) as offset (Velthuis et al., 2003) using STATA version 14. The susceptibility and infectiousness coefficients obtained from the analysis were used to calculate the transmission parameters by inserting them into the formulae described in Table 5.1. Note that in this case we observed the total outbreak in the sub-kebele and thus the regression coefficient estimates pertain to the final size of the outbreak and thus we directly estimate the reproduction ratio $\mathrm{R}$ rather than the transmission rate parameter $\beta$.

Vaccine efficacy for susceptibility (VEs) and infectiousness (VEi) were estimated using formula 1 and 2 as described by Halloran et al. (2010) and Aznar et al. (2011) and for this the four transmission parameters with their expression were defined (Table 5.1).

$$
\begin{aligned}
& V E s=1-\left(\frac{R u v}{R u u}\right)=1-\left(\frac{R v v}{R v u}\right) \\
& V E i=1-\left(\frac{R v u}{R u u}\right)=1-\left(\frac{R v v}{R u v}\right)
\end{aligned}
$$


Table 5.1 The fitted model to estimate LSD transmission rates in cattle populations with different levels of vaccination coverage in Mota town and Hulet Ejju Enessie district of Ethiopia.

\begin{tabular}{|c|c|c|}
\hline $\begin{array}{l}\text { Partial } R \\
\text { value }\end{array}$ & $\begin{array}{c}\log \beta=c 0+c 1^{*} \text { vaccination }+c 2 * f^{\text {fracVaccl }}{ }^{a} \\
\text { Description }\end{array}$ & Expression $^{b}$ \\
\hline Ruu & $\begin{array}{l}\text { Transmission from an unvaccinated to an } \\
\text { unvaccinated animal }\end{array}$ & $\mathrm{e}^{\mathrm{co}}$ \\
\hline Ruv & $\begin{array}{l}\text { Transmission from an unvaccinated to a } \\
\text { vaccinated animal }\end{array}$ & $\mathrm{e}^{\mathrm{c0}+\mathrm{c1}}$ \\
\hline$R v u$ & $\begin{array}{l}\text { Transmission from a vaccinated to an } \\
\text { unvaccinated animal }\end{array}$ & $\mathrm{e}^{\mathrm{c0}+\mathrm{c} 2}$ \\
\hline$R v v$ & $\begin{array}{l}\text { Transmission from a vaccinated to a } \\
\text { vaccinated animal }\end{array}$ & $\mathrm{e}^{\mathrm{c} 0+\mathrm{c1} 1+\mathrm{c} 2}$ \\
\hline
\end{tabular}

${ }^{a}$ Fraction of vaccinated among the infected.

${ }^{b}$ Relation between infection parameters and estimated coefficients of the model, where $\mathrm{c} 0$ is the estimated intercept and $c 1$ and $c 2$ are the estimated regression coefficients of the variables vaccination and fracVaccl respectively.

A vaccine with an efficacy of 0 was considered as not effective whereas a value of 1 was considered fully efficacious. Values of vaccine efficacies above 0.7 are considered 'good', whereas vaccine efficacies in the range of 0.3 to 0.7 are generally considered 'reasonable' (Halloran et al., 2010; Lu. et al., 2013). However, this interpretation of vaccine efficacy does not correspond to whether vaccination will reduce $R$ so that $R<1$, because whether $R<1$ also depends on the $R$ in the absence of vaccination.

The reproduction ratio in vaccinated animals was calculated by multiplying the effects of vaccination on susceptibility (exp(coefficient of the independent variable Vaccination)), and on infectiousness (exp(coefficient of the fraction of vaccinated among the infected)) and the intercept of the regression model. Whereas $R$ for unvaccinated was calculated from the exponent of the intercept only.

\subsection{Results}

\subsubsection{Questionnaire survey}

Based on the herd owner's response, the vaccination coverage at herd level was estimated to be $56.3 \%$. The public veterinary service vaccinated the majority (88.9\%) of the herds and more than $95 \%$ of the herds did not get routine prophylactic vaccination against LSD but were vaccinated just after the LSD index case was reported in a neighbouring kebele. More than $60 \%$ of the herd owners deemed the 
vaccine to be of low to very low efficacy in protecting against clinical LSD, however, almost all of them responded that the vaccine did not induce any adverse reaction after vaccination (Table 5.2).

Table 5.2 Ethiopian herd owners' opinion on LSD vaccine effectiveness and adverse reactions.

\begin{tabular}{lcccccc}
\hline Level & \multicolumn{3}{c}{ Vaccine effectiveness } & \multicolumn{3}{c}{ Vaccine adverse reactions } \\
\cline { 2 - 7 } & Frequency & $\%$ & Cum. \% & Frequency & $\%$ & Cum. \% \\
\hline Very high & 0 & 0 & 0 & 1 & 0.8 & 0.8 \\
High & 29 & 23.2 & 23.2 & 1 & 0.8 & 1.6 \\
Moderate & 20 & 16.0 & 39.2 & 0 & 0 & 1.6 \\
Low & 6 & 4.8 & 44 & 0 & 0 & 1.6 \\
Very low & 70 & 56.0 & 100 & 123 & 98.4 & 100 \\
\hline Total & $\mathbf{1 2 5}$ & $\mathbf{1 0 0}$ & $\mathbf{1 0 0}$ & $\mathbf{1 2 5}$ & $\mathbf{1 0 0}$ & $\mathbf{1 0 0}$ \\
\hline
\end{tabular}

\subsubsection{Follow-up study}

\subsubsection{Description of LSD occurrence and vaccination}

The follow-up study was undertaken in 10 sub-kebeles with 339 infected herds comprising a total of 2053 cattle of which 1304 (63.5\%) were vaccinated (Table 5.3). Herd size varied from $1(n=6)$ to $37(n=1)$ with an average of 6 and a median of 6 animals. About $95 \%$ of the herds had 10 or less animals. The study population consisted of $346(16.8 \%)$ calves, 263 (12.8\%) heifers, 227 (11.1\%) bulls, 490 (23.9\%) cows and 727 (35.4\%) oxen. Of the 2053 animals, 526 (25.6\%) were diagnosed with LSD, $233(31.1 \%)$ in the unvaccinated group and $293(22.5 \%)$ in the vaccinated group (Chi-square test: $p<0.001$ ). The PCR results confirmed the LSD infection in all ten subkebeles.

The multivariable population averaged model showed that herd did not contribute significantly to the total variance. Therefore multivariable logistic regression without random effects was performed which showed that the estimates and their significance were very similar to the random effects model. All variables remained significant in the multivariable analysis except herdtype but this variable confounded the estimates of location. Results show that vaccination significantly decreased the risk of LSD $(\mathrm{OR}=0.49,95 \% \mathrm{Cl}$ : 0.37 ; 0.64). Crossbreeds, males and older age were associated with increased risk to be LSD positive compared to their references and the interaction between vaccination and breed was significant. Vaccination is more efficient in crossbreed $(O R=0.49 * 0.43=0.21)$ than local breed (OR=0.49) animals (Table 5.4). 


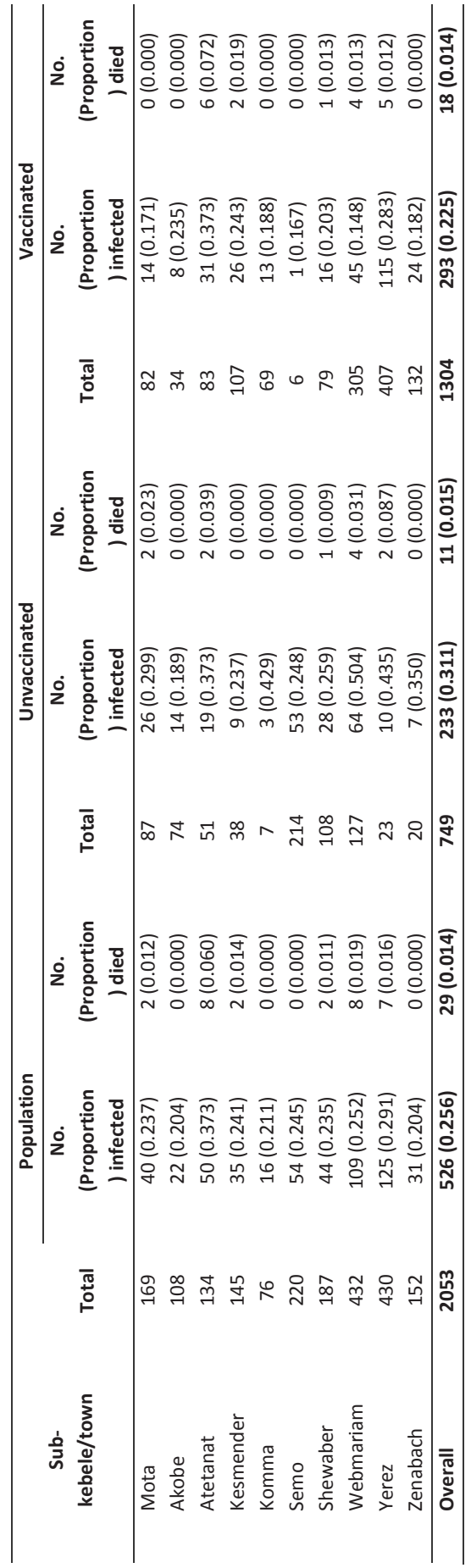




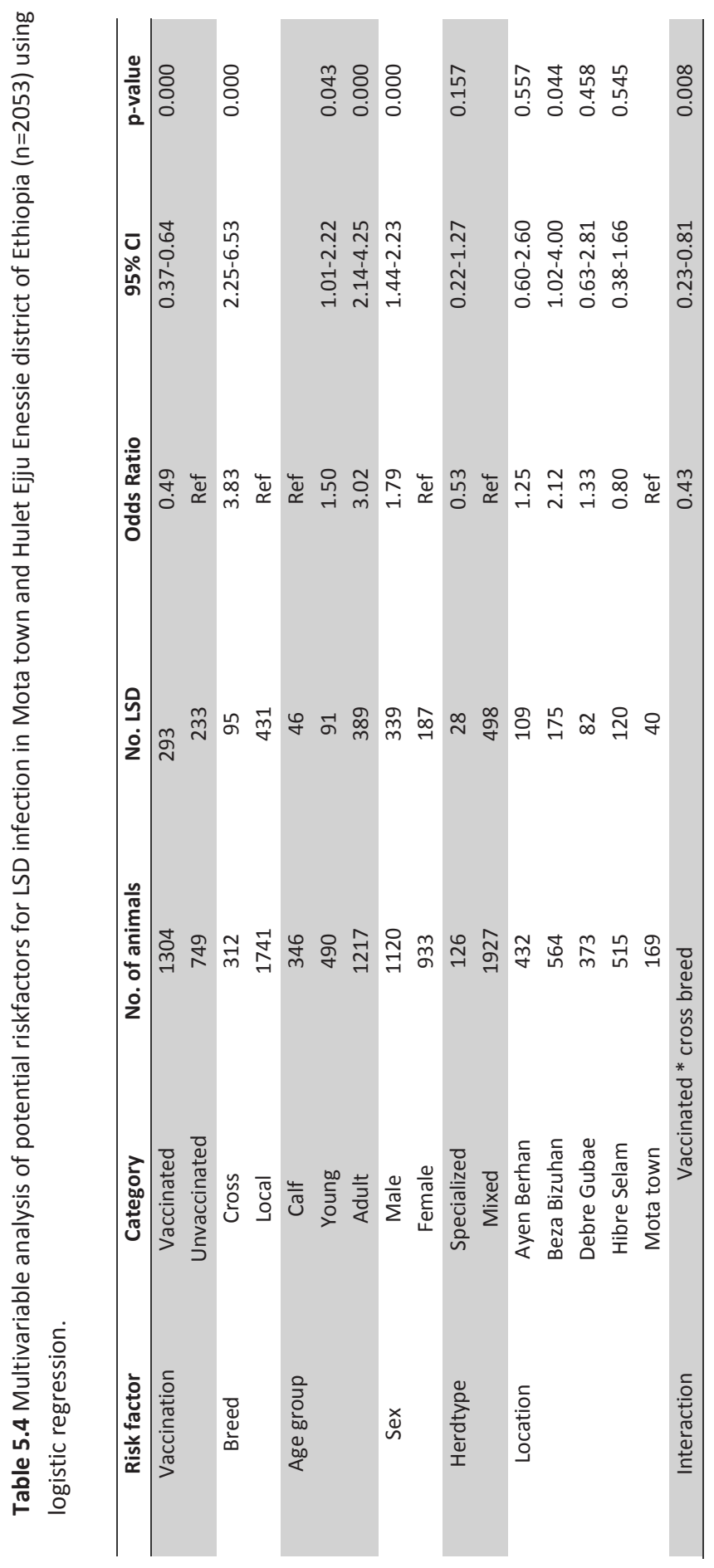




\subsubsection{LSD severity and vaccination}

The severity of LSD was assessed on a total of 480 clinically infected cattle (264 vaccinated and 216 unvaccinated). In unvaccinated animals, the majority of the affected animals (50.5\%) were categorized as severe and $9.7 \%$ fell in the mild category whereas in vaccinated animals these figures were $42.8 \%$ and $17.1 \%$ respectively (Table 5.5). The results of the multivariable ordered logistic model showed that only vaccination was significantly associated with a different (lower) severity score (Odds Ratio $(O R)=0.68,95 \%$ confidence interval $(\mathrm{Cl}): 0.49 ; 0.96)$. The test for the proportional odds assumption was not significant $(p=0.21)$ indicating that it is valid to report the OR as 0.68 . Furthermore, the predicted fraction showed that the probability of developing moderate and severe disease was slightly higher in unvaccinated animals (0.89) compared to vaccinated animals (0.84).

Table 5.5. LSD severity in vaccinated and unvaccinated cattle population $(n=480)$ of Ethiopia.

\begin{tabular}{lcccc}
\hline $\begin{array}{l}\text { Severity } \\
\text { level }\end{array}$ & \multicolumn{2}{c}{ Vaccinated } & \multicolumn{2}{c}{ Unvaccinated } \\
\cline { 2 - 5 } & Number & $\begin{array}{c}\text { Proportion } \\
\text { in \% }\end{array}$ & Number & $\begin{array}{c}\text { Proportion } \\
\text { in \% }\end{array}$ \\
\hline Mild & 45 & 17.1 & 21 & 9.7 \\
Moderate & 106 & 40.2 & 86 & 39.8 \\
Severe & 113 & 42.8 & 109 & 50.5 \\
\hline Total & $\mathbf{2 6 4}$ & $\mathbf{1 0 0}$ & $\mathbf{2 1 6}$ & $\mathbf{1 0 0}$ \\
\hline
\end{tabular}

\subsubsection{LSD vaccine efficacy with respect to transmission}

The multivariable GLM analysis showed that both the susceptibility $(\exp (b)=$ $0.54,95 \% \mathrm{Cl}: 0.44 ; 0.66)$ and infectiousness ( $\exp (\mathrm{b})=1.83,95 \% \mathrm{Cl}: 1.28 ; 2.61)$ effects of the vaccine are significant and thus the effects are a reduction in susceptibility by a factor 2 and an increase in infectiousness by a factor 2 (Table 5.6).

A 0.46 vaccine efficacy for susceptibility and -0.83 for infectiousness recorded in this study were obtained by inserting the corresponding estimated partial reproduction ratios ( $\mathrm{Ruu}=1.21$, $\mathrm{Ruv}=0.65$, $\mathrm{Rvu}=2.22$ and $\mathrm{Rvv}=1.19$ ) into formula 1 and 2 (Table 5.1 and 5.6).

The estimated reproduction ratios for vaccinated and unvaccinated cattle were almost equal: 1.19 (95\% Cl: 1.02-1.39) and 1.21 (95\% Cl: 1.01-1.46). The 0.98 (95\% $\mathrm{Cl}: 0.73-1.33)$ reduction in $\mathrm{R}$ by vaccination was not significantly different from $1(p=0.92)$. 
Table 5.6 Analysis of the effect of vaccination on susceptibility and infectiousness of LSD in Mota town and Hulet Ejju Enessie district of Ethiopia ( $n=2053$ ) using GLM.

\begin{tabular}{llcccc}
\hline Variable & $\begin{array}{c}\text { Susceptibility/i } \\
\text { nfectiousness }\end{array}$ & $\begin{array}{c}\text { Coefficient } \\
\text { (b) }\end{array}$ & $\begin{array}{c}\text { Effect } \\
(\mathbf{e x p}(\mathbf{b}))\end{array}$ & $\mathbf{9 5 \% \mathbf { C l }}$ & $\begin{array}{c}\mathbf{p}- \\
\text { value }\end{array}$ \\
\hline Vaccination & Susceptibility & -0.62 & 0.54 & $0.44-0.66$ & 0.000 \\
FracVaccl $^{\mathrm{a}}$ & Infectiousness & 0.60 & 1.83 & $1.28-2.61$ & 0.001 \\
Constant & & 0.19 & 1.21 & $1.00-1.46$ & 0.045 \\
\hline
\end{tabular}

${ }^{\text {aFraction }}$ of vaccinated among the infected in each population (= sub-kebele).

\subsection{Discussion}

LSD vaccine breakdown and a concomitant morbidity are reported in Ethiopian cattle since 1993 (Carn, 1993) while vaccination with KS1 O-180 vaccine is the major control method in the country. However, the efficacy of KS1 0-180 virus strain vaccine against natural LSD infections under field conditions and its impact on the transmission and severity of the disease is largely unknown and both are estimated in this paper.

\subsubsection{Questionnaire survey}

The questionnaire survey shows that in almost all study districts no regular vaccination program for LSD is applied. This is related to the long time (5 or more years) interval between LSD epidemics (Woods, 1988) and resource limitation. LSD vaccination is usually initiated by the appearance of an index case in an area. Therefore, vaccination for LSD is commonly carried out at the face of the outbreak to control the disease occurrence. However, vaccinating animals during an outbreak may aggravate the transmission of LSD due to iatrogenic transmission from healthy looking, incubating animals to susceptible animals (Hunter and Wallace, 2001). The survey also showed that most of the vaccinations were provided by the public veterinary service. This clears out the suspect that the vaccine failure might be related to the administration of the vaccine by incompetent practitioners (and that apply LSD vaccination illegally).

Vaccination coverage is an important issue in disease control. Cattle populations with low vaccination coverage are assumed to remain at higher risk for the disease. The $56.3 \%$ vaccination coverage at herd level estimated in this study is low given that the vaccine is provided free of charge. The reason for low coverage might be related to owner's belief that the vaccine is not protective. More than $60 \%$ 
of the herd owners interviewed in the questionnaire survey reported low effectiveness of KS1 0-180 vaccine in protecting cattle against clinical LSD confirming the estimated poor performance of the vaccine (Ayelet et al., 2013; Gelaye et al., 2015). However, the low vaccination coverage is not related to vaccine adverse effects as almost all respondents did not indicate any adverse effect. This is in agreement with what Gelaye et al. (2015) reported for the vaccine. However, in other countries adverse reactions in cattle vaccinated with sheep pox and Neethling virus based vaccine have been reported like swelling on the injection site and developing active LSD (Weiss, 1968; Yeruham et al., 1994; Ben-Gera et al., 2015; Abutarbush et al., 2016).

\subsubsection{Follow-up study}

The $22.5 \%$ morbidity in vaccinated animals recorded in the follow-up study is comparable to $23.8 \%$ morbidity reported in central Ethiopia in cattle vaccinated with Kenyan sheep pox vaccine strain (Ayelet et al., 2013). However, a much lower morbidity of $4.7 \%$ (Abutarbush, 2014), 11\% (Brenner et al., 2009) and 1.6\% (BenGera et al., 2015) were recorded in vaccinated cattle of Jordan and Israel. This difference might be attributed to the difference in the quality of the vaccine used, vaccination coverage, management system, environment or climate difference of the areas where the animals are kept.

The factors age group, breed, sex, herdtype and location were included into the logistic regression model to adjust the estimate of vaccination. The adjusted odds ratio for vaccination was 0.49 which indicates that vaccination is protective for LSD. Unvaccinated animals have 2.04 (1/0.49) times higher odds to acquire LSD than vaccinated ones. The interaction between vaccination and breed was significant and it revealed that vaccination was more efficient in crossbreed $(O R=0.21)$ than local breed $(O R=0.49)$ animals. This might be related with the more susceptibility nature of Holstein-Zebu cross to LSD than pure local Zebu animals (Davies 1991; OIE, 2010). Possible confounding factors which are not measured in this study include movement of animals and vector density. No animal movement restriction was applied in the study area; animals move freely from area to area. This practice was similar in all study kebeles and for both vaccinated and unvaccinated animals. Vector density is also assumed to be similar in all study kebeles because they are located in the same geographical area with similar weather conditions and altitude and on top of that they are all within the range of the insect flight zone.

Vaccination was associated with less severe LSD symptoms. This finding is in agreement with the observation of Abutarbush (2014) who reported a considerable change in feed intake and milk production, fever, and a longer duration 
of illness in the majority of unvaccinated cattle as compared to vaccinated cattle. Hence, LSD vaccination reduces disease severity and as consequence it may prevent part of the production loss due to LSD. Increased vaccine dose is claimed to improve the protective efficacy of the vaccine. Ben-Gera et al. (2015) reported a low incidence $(1.85 \%)$ in cattle vaccinated with a 10 fold increased dose of RM65 vaccine. The regular vaccine dose used to immunize cattle against LSD in Ethiopia is 10 fold compared what used to immunize sheep and goat. For cattle, LSD vaccine contains $10^{3.5} \mathrm{TCID}_{50}$ attenuated virus per field dose while for sheep and goat it is, $10^{2.5} \mathrm{TCID}_{50}$ per dose (NVI, 2010).

The vaccine efficacy of 0.46 as estimated for susceptibility is within the 'reasonable' efficacy range of 0.3 to 0.7 (Halloran et al., 2010; Lu et al., 2013).This indicates that vaccination reduces susceptibility to LSD 1.85 times (1/(1-0.46)). However, vaccinated infected animals are 1.83 times more infectious than unvaccinated infected ones. This is contradictory from what is expected from a vaccine. The increased infectiousness might be related with disease management practices. In the usual management practice, diseased animals are isolated and penned separately from healthy animals. However, the situation in vaccinated LSD affected animals is different, they are less diseased (not easily noticed) and thus remain longer in the herd (not isolated or removed) while they are infectious. This condition might be favourable for the transmission of the virus. Therefore, in this regard, animal disease management might contribute to increased infectiousness. However, this finding needs further investigation because the disease management and other factors which can influence the infectiousness were not under control. In general, the gain in decreasing susceptibility in vaccinated cattle is cancelled out by almost the same increment of infectiousness and this indicates that KS1 0-180 vaccine is not effective in controlling LSD in cattle populations. The overall low efficacy of the vaccine substantiates the previous findings that vaccination against LSD does not provide protection from clinical disease (Ayelet et al., 2013; Abutarbush, 2014; Gari et al., 2015). Most LSD vaccines currently available, except the homologous Neethling vaccine, provide poor protection against LSD transmission (Brenner et al., 2009; Somasundaram, 2011; Tuppurainen et al., 2014; Ben-Gera et al., 2015), which is a challenge for the control of the disease.

Although vaccinating cattle against LSD is considered the main control option in resource poor countries like Ethiopia, little is known about the effect of vaccination on the disease dynamics. In the current study, the estimated reproduction ratios were 1.21 and 1.19 for unvaccinated and vaccinated cattle, respectively. In both cases $R$ is greater than 1 and confirms that LSD virus can spread in cattle populations, regardless of their vaccination status, and can cause a major 
outbreak. This shows that vaccination with KS1 0-180 vaccine alone cannot eliminate the disease from a cattle population. Thus, a more competent LSD vaccine and other additional measures, like movement control, detection and removal of infected animals, are needed to bring the reproduction ratio to below 1.0.

An observational study was chosen for this study because it is less costly and enables to assess the performance of the vaccine under real-life circumstances, including the complex and not easily controllable exposure to LSDV due to the insect vectors involved. Important confounders were measured and equal exposure risk of vaccinated and unvaccinated animals were assumed. Furthermore, the study design avoids the ethical problem of using a placebo when an approved vaccine is available (Torvaldsen and McIntyre, 2002).

Observational studies are prone to potential biases due to its uncontrolled nature. The biases may be related to selection, misclassification of cases, confounding factors, dealing with the impact of unknown or unmeasured factors (Dohoo et al., 2003), missing information, and non-comparability of groups. The distribution of potentially confounding variables among the study groups and other variables which were not considered might also be a source of bias. Another limitation to this study is related to the severity assessment; subjectivity might be somehow involved in allocating affected animals into different categories and on few occasions the observer might have been unblinded for the vaccination status of the animal because the owner might have complained about the poor efficacy of the vaccine. We assumed that exposure to infection was equal in both vaccinated and unvaccinated animals, that all important confounders were measured and adjusted for by the model used. Considering these limitations, the results reported here should be interpreted carefully.

\subsection{Conclusion}

The results of our study showed that KS1 0-180 strain vaccine reduces susceptibility of cattle to LSD but it also increases infectiousness by about the same amount, partially because animals with less severe disease signs may remain undetected in the herd for longer periods. Generally, the vaccine has poor efficacy in protecting cattle populations against LSD, neither by direct clinical protection nor by reducing transmission. Therefore, the prevailing situation dictates the urgent need of a competent LSD vaccines development to control LSD in endemic countries and to halt its current spread to free countries and continents. 


\section{Acknowledgments}

The authors would like to thank NUFFIC (Netherlands Organization for International Cooperation in Higher Education) for funding the study. We would like to extend our gratitude to the National Animal Health Diagnostic and Investigation Centre, Ethiopia for assistance in field work and laboratory confirmation of the disease. We also thank district animal health professionals and the herd owners for their kind collaboration in collecting and providing information for the study.

\section{References}

Abutarbush, S.M., 2014. Efficacy of vaccination against lumpy skin disease in Jordanian cattle. Vet. Rec. 175.

Abutarbush, S.M., Hananeh, W.M., Ramadan, W., Al Sheyab, O.M., Alnajjar, A.R., Al Zoubi, I.G., Knowles, N.J., Bachanek-Bankowska, K., Tuppurainen, E.S., 2016. Adverse reactions to field vaccination against lumpy skin disease in Jordan. Transbound. Emerg. Dis. 63, e213-219.

APHRD, 2012. Ethiopia Animal Health Yearbook 2011 Animal and Plant Health Regulatory Directorate (APHRD), Addis Ababa, Ethiopia.

Ayelet, G., Abate, Y., Sisay, T., Nigussie, H., Gelaye, E., Jemberie, S., Asmare, K., 2013. Lumpy skin disease: preliminary vaccine efficacy assessment and overview on outbreak impact in dairy cattle at Debre Zeit, central Ethiopia. Antivir. Res. 98, 261-265.

Aznar, I., McGrath, G., Murphy, D., Corner, L.A., Gormley, E., Frankena, K., More, S.J., Martin, W., O'Keeffe, J., De Jong, M.C., 2011. Trial design to estimate the effect of vaccination on tuberculosis incidence in badgers. Vet. Microbiol. 151, 104-111.

Ben-Gera, J., Klement, E., Khinich, E., Stram, Y., Shpigel, N.Y., 2015. Comparison of the efficacy of Neethling lumpy skin disease virus and x10RM65 sheep-pox live attenuated vaccines for the prevention of lumpy skin disease - The results of a randomized controlled field study. Vaccine 33, 4837-4842.

Brenner, J., Bellaiche, M., Gross, E., Elad, D., Oved, Z., Haimovitz, M., Wasserman, A., Friedgut, O., Stram, Y., Bumbarov, V., Yadin, H., 2009. Appearance of skin lesions in cattle populations vaccinated against lumpy skin disease: statutory challenge. Vaccine 27, 1500-1503.

Carn, 1993. Control of capripoxvirus infections. Vaccine 11, 1275-1279.

Dohoo, I., Martin, W., Stryhn, H., 2003. Veterinary Epidemiologic Research. AVC Inc. Charlottetown, Canada. pp. 1-21. 
Davies, F.G., 1991. Lumpy skin disease, an African capripox virus disease of cattle. Br. Vet. J. 147, 489-503.

De Jong, M.C.M., 1994. Experimental quantification of vaccine induced reduction in virus transmission reduction. Vaccine 12, 761-766.

Diekmann, O., Heesterbeek, J.A.P., Metz, J.A.J., 1990. On the definition and the computation of the basic reproduction ratio $\mathrm{RO}$ in models for infectious diseases in heterogeneous populations J. Math. Biol. 28, 365-382.

Gari, G., Abie, G., Gizaw, D., Wubete, A., Kidane, M., Asgedom, H., Bayissa, B., Ayelet, G., Oura, C.A., Roger, F., Tuppurainen, E.S., 2015. Evaluation of the safety, immunogenicity and efficacy of three capripoxvirus vaccine strains against lumpy skin disease virus. Vaccine 33, 3256-3261.

Gari, G., Bonnet, P., Roger, F., Waret-Szkuta, A., 2011. Epidemiological aspects and financial impact of lumpy skin disease in Ethiopia. Prev. Vet. Med. 102, 274283.

Gari, G., Waret-Szkuta, A., Grosbois, V., Jacquiet, P., Roger, F., 2010. Risk factors associated with observed clinical lumpy skin disease in Ethiopia. Epidemiol. Infect. 138, 1657-1666.

Gelaye, E., Belay, A., Ayelet, G., Jenberie, S., Yami, M., Loitsch, A., Tuppurainen, E., Grabherr, R., Diallo, A., Lamien, C.E., 2015. Capripox disease in Ethiopia: Genetic differences between field isolates and vaccine strain, and implications for vaccination failure. Antivir. Res. 119, 28-35.

Gelaye, E., Lamien, C.E., Silber, R., Tuppurainen, E.S., Grabherr, R., Diallo, A., 2013. Development of a cost-effective method for capripoxvirus genotyping using snapback primer and dsDNA intercalating dye. PloS One 8, e75971.

Halloran, M.E., Longini, J.I., Struchiner, C.J., 2010. Design and Analysis of Vaccine Studies. Springer, New York. pp. 19-45.

Halloran, M.E., Struchiner, C.J., Longini, J.I., 1997. Study designs for evaluating different efficacy and effectiveness aspects of vaccines. Am. J. Epidemiol. 146,789-803.

Heffernan, J.M., Smith, R.J., Wahl, L.M., 2005. Perspectives on the basic reproductive ratio. J. R. Soc. Interface 2, 281-293.

Hunter, P., Wallace, D., 2001. Lumpy skin disease in southern Africa: a review of the disease and aspects of control. J. S. Afr. Vet. Ass. 72, 68-71.

Kumar, S.M., 2011. An outbreak of lumpy skin disease in a Holstein dairy herd in Oman: A clinical report Asian J. Anim. Vet. Adv. 6, 851-859.

Longini, Jr., I.M., Sagatelian, K., Rida, W.N., Halloran, M.E., 1998. Optimal vaccine trial design when estimating vaccine efficacy for susceptibility and infectiousness from multiple populations. Stat. Med. 17, 1121-1136. 
Lu, Z., Schukken, Y.H., Smith, R.L., Mitchell, R.M., Gröhn, Y.T., 2013. Impact of imperfect Mycobacterium avium subsp. paratuberculosis vaccines in dairy herds: A mathematical modeling approach. Prev. Vet. Med. 108, 148-158.

Mebratu, G.Y., Kassa, B., Fikre, Y., Berhanu, B., 1984. Observation on the outbreak of lumpy skin disease in Ethiopia Rev. Elev. Méd. Vét. Pays Trop. 37, 395-399.

NVI, 2010 Product catalogue. User manual for lumpy skin disease vaccine. Issue In: National Veterinary Institute (Ed.), 12.

OIE, 2010. Manual of diagnostic tests and vaccines for terrestrial animals, chapter 2.4.14, Lumpy disease. OIE, Paris. http://web.oie.int/eng/normes/MMANUAL/A Index.htm. Accessed 26 February 2016.

OIE, 2015. Manual of diagnostic tests and vaccines for terrestrial animals, volume 1, chapter 1.1.6, principles of veterinary vaccine production. OIE, Paris. http://www.oie.int/fileadmin/Home/eng/Health_standards/tahm/1.01.06 _VACCINE_PRODUCTION.pdf. Accessed 8 March 2016.

OIE, 2016. OIE-Listed diseases, infections and infestations in force in 2016. OIE, Paris. http://www.oie.int/animal-health-in-the-world/oie-listed-diseases-2016/. Accessed 8 March 2016).

Quinn, P.J., Carter, M.E., Markey, B., Carter, G.R., 1999. Clinical Veterinary Microbiology. Elsevier Ltd, London. pp. 486-495.

Quinn, P.J., Markey, B.K., Carter, M.E., Donnelly, W.J., Leonard, F.C., 2002. Veterinary Microbiology and Microbial Disease. Blackwell Science, Great Britain. pp. 335-342.

Radostits, O.M., Gay, C.C., Hinchcliff, K.W., Constable, P.D., 2007. Veterinary Medicine: A Textbook of the Diseases of Cattle, Sheep, Pigs, Goats and Horses. Sounders Elsevier, Spain. pp. 1424-1426.

Smith, P.G., Rodrigues, L.C., Fine, P.E., 1984. Assessment of the protective efficacy of vaccines against common diseases using case-control and cohort studies. Int. J. Epidemiol. 13, 87-93.

Somasundaram, M.K., 2011. An outbreak of lumpy skin disease in a Holstein dairy herd in Oman: a clinical report. Asian J. Anim. Vet. Adv. 6, 851-859.

Tasioudi, K.E., Antoniou, S.E., Iliadou, P., Sachpatzidis, A., Plevraki, E., Agianniotaki, E.I., Fouki, C., Mangana-Vougiouka, O., Chondrokouki, E., Dile, C., 2016. Emergence of lumpy skin disease in Greece, 2015. Transbound. Emerg. Dis. 63, 260-265.

Torvaldsen, S., Mclntyre, P.B., 2002. Observational methods in epidemiologic assessment of vaccine effectiveness. Commun. Dis. Intell. Q. Rep. 26, 451457. 
Tuppurainen, E.S., Oura, C.A., 2012. Review: lumpy skin disease: an emerging threat to Europe, the Middle East and Asia. Transbound. Emerg. Dis. 59, 40-48.

Tuppurainen, E.S., Pearson, C.R., Bachanek-Bankowska, K., Knowles, N.J., Amareen, S., Frost, L., Henstock, M.R., Lamien, C.E., Diallo, A., Mertens, P.P., 2014. Characterization of sheep pox virus vaccine for cattle against lumpy skin disease virus. Antivir. Res. 109, 1-6.

Tuppurainen, E.S., Venter, E.H., Shisler, J.L., Gari, G., Mekonnen, G.A., Juleff, N., Lyons, N.A., De Clercq, K., Upton, C., Bowden, T.R., Babiuk, S., Babiuk, L.A., 2017. Review: capripoxvirus diseases: current status and opportunities for control. Transbound. Emerg. Dis. 64, 729-745.

Tuppurainen, E.S.M., Venter, E.H., Coetzer, J.A.W., 2005. The detection of lumpy skin disease virus in samples of experimentally infected cattle using different diagnostic techniques. Onderstepoort J. Vet. Res. 72, 153-164.

Van der Goot, J.A., Van Boven, M., Koch, G., de Jong, M.C.M., 2007. Variable effect of vaccination against highly pathogenic avian influenza (H7N7) virus on disease and transmission in pheasants and teals. Vaccine 25, 8318-8325.

Velthuis, A.G.J., De Jong, M.C.M., Kamp, E.M., Stockhofe, N., Verheijden, J.H.M., 2003. Design and analysis of an Actinobacillus pleuropneumoniae transmission experiment. Prev. Vet. Med. 60, 53-68.

WAHID, 2016. Summary of Immediate notifications and Follow-ups. World Animal Health Information Database (WAHID).

Weiss, K.E., 1968. Lumpy skin disease virus. Virol. Monogr. 3, 111-131.

Woods, J.A., 1988. Lumpy skin disease- A review. Trop. Anim. Hlth Prod. 20, 11-17.

Yeruham, I., Perl, S., Nyska, A., Abraham, A., Davidson, M., Haymovitch, M., Zamir, O., Grinstein, H., 1994. Adverse reactions in cattle to a capripox vaccine. Vet. Rec.135, 330-332. 


\section{Economic impact of lumpy skin disease and cost effectiveness of vaccination for the control of outbreaks in Ethiopia}

\footnotetext{
Wassie Molla ${ }^{1,2}$, Mart C.M. de Jong ${ }^{1}$, Getachew Gari ${ }^{3}$, Klaas Frankena ${ }^{1}$

${ }^{1}$ Quantitative Veterinary Epidemiology, Wageningen University \& Research, Droevendaalsesteeg 1, 6708 PB Wageningen, The Netherlands

${ }^{2}$ Faculty of Veterinary Medicine, University of Gondar, P.O. Box 196, Gondar, Ethiopia

${ }^{3}$ National Animal Health Diagnostic and Investigation Centre (NAHDIC), Sebeta, Ethiopia
}

Preventive Veterinary Medicine (2017) 147:100-107 DOI: http://dx.doi.org/10.1016/j.prevetmed.2017.09.003 


\begin{abstract}
Lumpy skin disease (LSD), an infectious viral disease of cattle, causes considerable financial losses in livestock industry of affected countries. A questionnaire survey with the objectives of determining direct economic losses of LSD (mortality loss, milk loss, draft loss) and treatment costs (medication and labour cost) per affected herd, and assessing the cost effectiveness of vaccination as a means for LSD control was carried out in the central and north-western parts of Ethiopia. From a total of 4430 cattle (in 243 herds) surveyed, 941 animals (in 200 herds) were reported to be infected. The overall morbidity and mortality at animal level were $21.2 \%$ and $4.5 \%$, and at herd level these were $82.3 \%$ and $24.3 \%$. There was a significant difference in animal level morbidity and mortality between categories of animals. Over $94 \%$ of the herd owners ranked LSD as a big or very big problem for cattle production. A large proportion (92.2\%) of the herd owners indicated that LSD affects cattle marketing. A median loss of USD 375 (USD 325 in local Zebu and USD 1250 in Holstein-Friesian local Zebu cross cattle) was estimated per dead animal. Median losses per affected lactating cow were USD 141 (USD 63 in local Zebu cows and USD 216 in Holstein-Friesian local Zebu cross cows) and, USD 36 per affected ox. Diagnosis and medication cost per affected animal were estimated at USD 5. The median total economic loss of an LSD outbreak at herd level was USD 1176 (USD 489 in subsistence farm and USD 2735 in commercial farm). At herd level, the largest component of the economic loss was due to mortality (USD 1000) followed by milk loss (USD 120). LSD control costs were the least contributor to herd level losses. The total herd level economic losses in the commercial farm type were significantly higher than in the subsistence farm type. The financial analysis showed a positive net profit of USD 136 (USD 56 for subsistence farm herds and USD 283 for commercial herds) per herd due to LSD vaccine investment. It should be noted that only the noticeable direct costs and treatment costs associated with the disease were considered in the study. Generally, vaccination is economically effective and should be encouraged.
\end{abstract}

Key words: LSD outbreak, Morbidity, Mortality, Economic loss, Vaccination, Ethiopia 


\subsection{Introduction}

Lumpy skin disease (LSD) is a severe systemic disease of cattle caused by the lumpy skin disease virus, which belongs to the genus Capripoxvirus, family Poxviridae. It is characterized by fever, nodular lesions on the skin and mucous membranes and lymphadenopathy (Murphy et al., 1999; Radostits et al., 2007). The morbidity during LSD outbreaks varies greatly from $5 \%$ to $100 \%$ depending on the immune status of the host and the abundance of arthropod vectors (Woods, 1988; Tuppurainen and Oura, 2012). LSD mortality is generally low (usually less than 5\%) but occasionally may reach 20\% (Woods, 1988; Babiuk et al., 2008; OIE, 2010). LSD is associated with reduction in milk production, temporary or permanent sterility in bulls and cows, weight loss, draft power loss, abortion, damage to hides and death. Disease control and eradication measures such as vaccination campaigns, removal of affected animals, biosecurity are costly (Woods, 1988; Radostits et al., 2007; Babiuk et al., 2008; OIE, 2010; Tuppurainen and Oura, 2012). For example in Israel the control of the initial LSD outbreak costed USD 750,000, and the indirect financial loss associated with compulsory animal movement restrictions was also significant (AU-IBAR, 2013). The economic importance of the disease is also due to convalescence of several months (Murphy et al., 1999). The World Organization for Animal Health (OIE) categorized LSD as a notifiable disease because of its substantial economic impact (Tuppurainen and Oura, 2012; OIE, 2015). Because of these considerable financial losses and the international trade restrictions on live animals and their products, LSD is one of the most important infectious diseases in countries where it is endemic.

Livestock is an important sector in Ethiopia's economy as it contributes $35.6 \%$ to the agricultural Gross Domestic Product (GDP), equivalent to $16.5 \%$ of the national GDP (Metaferia et al., 2011), and 37 to $87 \%$ to the household incomes (GebreMariam et al., 2010). The contribution of livestock to the annual foreign exchange earnings amounts to $12 \%$ (NBE, 2014). Households keep cattle for multiple purposes: milk production, draft power, beef production, manure for fuel and fertilizer, and breeding (GebreMariam et al., 2010; Negassa et al., 2011). The total cattle population of Ethiopia is estimated to be about 57 million heads (CSA, 2015). The benefit that cattle could have for the country is not attained for several reasons and one important reason is animal disease. LSD stands among the major diseases that limit the productivity of the cattle population (Gari et al., 2011; APHRD, 2012). LSD was restricted to Africa and Middle East countries for decades, but recently it is spreading unusually beyond its territory into Europe and other Asian countries and increasingly becomes a risk for the livestock industry in these continents 
(Tuppurainen et al., 2015; Tasioudi et al., 2016; WAHIS, 2016). In Ethiopia, LSD was first observed in 1981 in the north-western part of the country (Mebratu et al., 1984). However, it has now spread to almost all regions and agro-ecological zones of the nation with seroprevalence ranging from $23-31 \%$ at animal level and $26-64 \%$ at herd level (Gari et al., 2010, 2012). The infection was reported to cause $33.93 \%$ and $13.41 \%$ morbidity and $7.43 \%$ and $1.25 \%$ mortality in Holstein-Friesian cross bred and local Zebu cattle, respectively (Gari et al., 2011).

Knowledge of disease impact is essential when deciding on the level of expenditure that can be justified for a disease control programme (Knight-Jones and Rushton, 2013). The economic impact of LSD can be largely influenced by the methods used to control and eradicate outbreaks. In general, LSD prevention and control programmes are based on one or more of the following three elements: routine vaccination, stamping-out and movement restriction (Davies, 1991; Carn, 1993; Horst et al., 1999). The main LSD prevention and control scheme in Ethiopia is through vaccination. Vaccination costs depend on the number of animals vaccinated, vaccine cost, vaccination frequency, and labour and distribution costs (Horst et al., 1999). In Ethiopia, vaccination cost is borne by the government, i.e. vaccines are provided free of charge to the livestock owners.

Disease impacts are generally easy to identify but may be difficult to quantify. Disease outbreaks often have broad, long-term effects on livestock industry. The costs of animal disease can roughly be divided into direct costs, which include losses related to animal illness, death and less immediate impacts such as reduced fertility, and indirect costs, which encompass control costs, losses in trade and other revenues (Rushton, 2009; Oxford-Analytica, 2012). Understanding the impact of animal disease and assessing its losses is useful for policy makers and farmers who may weigh the losses against the costs of disease control each at their own level (Pritchett et al., 2005). There has been very limited work carried out on the financial analysis of herd-level control of LSD. Therefore, the objectives of this study were to determine the direct financial losses of LSD related to milk loss, draft power loss, mortality and indirect losses due to treatment, and to assess the cost effectiveness of vaccination as a means of LSD control.

\subsection{Materials and methods}

\subsubsection{Study design and population}

A questionnaire survey targeted to assess the economic impact of LSD was carried out in the central and north-western parts of Ethiopia (Figure 6.1). In central part, it was undertaken in Ada'a, Sebeta Hawas, Ambo, Dendi, Debrelibanos, Kuyu and Hidabu Abote districts in Oromia National Regional State. In north-western part, 
the data were collected from Dejen, Gozamen, Hulet Ejju Enessie and Jabitenan districts in Amhara National Regional State. Furthermore, another five commercial dairy farms (Selale Dairy Development PLC at Muketuri, Aser at Ecoefobabo, Sululta; Selam Children Village in Addis Ababa, Holeta dairy cattle genetic improvement nucleus farm and Holeta agricultural research centre farm at Holeta) were included in the study.

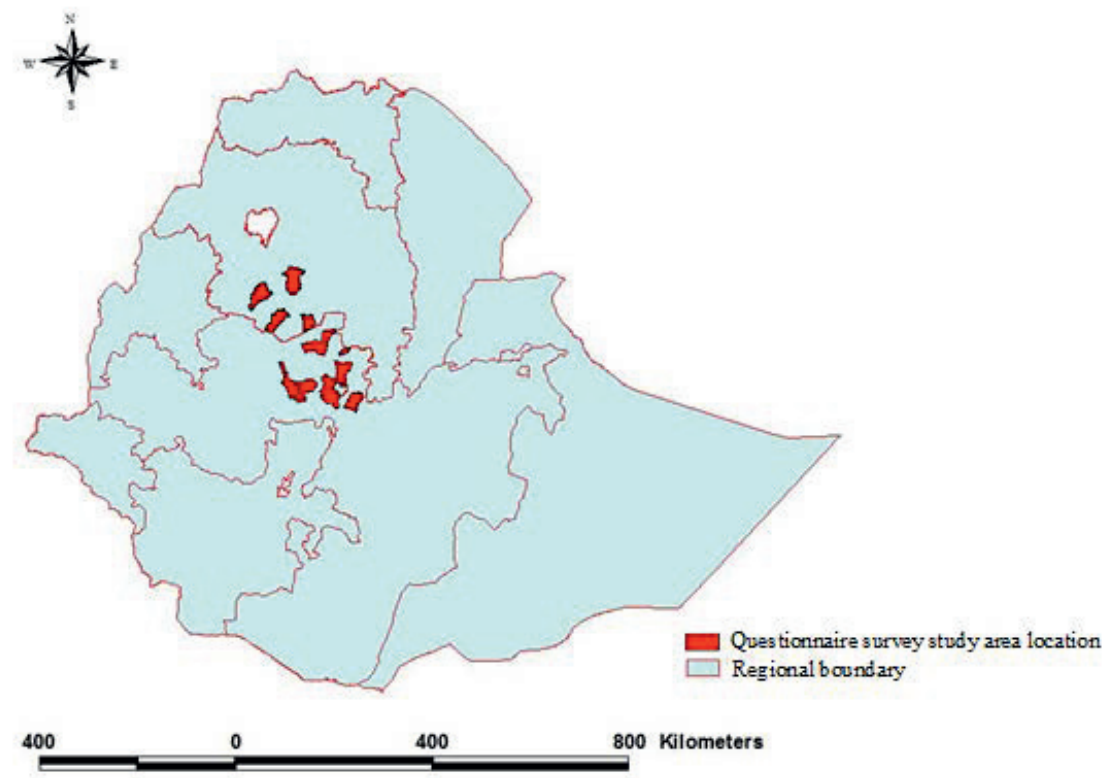

Figure 6.1. Map of Ethiopia showing the area and the location of 243 cattle farms included in the study of the economic impact of lumpy skin disease (2014/15).

The livestock production systems in the study area can be classified into two broad categories: subsistence crop-livestock production and commercial dairy production. In the subsistence production system the small holding farms are mainly kept for draft power, milk and meat production (Mengistu, 2003) and the composition of the herd is dominated by local Zebu cattle. The commercial dairy farms are market oriented and include medium (10-50 animals) to large-scale ( $>50$ animals) farms of crossbred Zebu with Holstein-Friesian. They are mostly located around peri-urban and urban areas practicing intensive and semi-intensive production (Mengistu, 2003). Milk and calf production are the main source of income. 


\subsubsection{Data collection}

The questionnaire survey was undertaken from October 2014 to May 2015. The time span for the financial analysis was one year i.e. May 2014 to April 2015. A total of 243 herd owners from 15 districts (comprising 34 kebeles and 5 farms) enrolled in the study, a number close to numbers used in comparable studies (Jemberu et al., 2014; Jibat et al., 2016; Chenais et al., 2017). Kebele is the smallest administrative division in Ethiopia. The districts were selected based on the occurrence of an LSD outbreak and three kebeles were randomly selected from each of 10 districts, four kebeles from one district, 2 farms from 1 district and 1 farm each from the other 3 districts. From each kebele, five to eight herd owners that were willing to participate were interviewed. The data were collected by face to face interview using the local language. An oral consent to use the data for scientific research was obtained from each participating herd owner before the interview started.

The questionnaire was designed primarily to record the magnitude of production losses, mortality, and cost of control for LSD in several categories of bovines in a herd (a group of cattle owned by a household or an organization), and perception of farmers on livelihood impact and its influence on cattle marketing during the outbreak period. The farmer's ability to identify LSD infection was crosschecked by enquiring about the main epidemiological and clinical features of LSD. If the herd owner's description was consistent with the classical clinical signs and epidemiologic features of LSD (nodular lesions on skin and mucosal surface, enlargement of superficial lymph nodes, swelling of the limb or the lower body, discharge from eyes, nostrils and mouth, reduced milk production in lactating cows, depression, morbidity varying from $5-45 \%$ and mortality less than $10 \%$ ) (FAO, 2010), they were considered to know the disease and the interview was continued. Farmers were also asked to estimate the daily milk production of their cattle before and after infection, the duration of infection, the milk price per litre, the renting price of an ox, the market value of animal, labour time lost for an animal getting treated and wage of a daily labourer. Commercial farms and some of subsistence herd owners estimated the volume of the daily milk produced in litres. However, the majority of subsistence herd owners estimated the volume of milk produced by each LSD affected cow using the local container (gourds or bucket) which normally is used for milking. This was later converted to litre after filling the container with water to the level indicated by the owner and measured using a graduated jug. Additional information such as treatment and vaccination cost were collected from veterinary practitioners. Financial information was collected first in Ethiopian currency (Birr) and later converted to USD at an exchange rate of 20 Birr = USD 1 (8 October, 2014). 


\subsubsection{Estimation of economic losses}

The economic impact of LSD was determined by an estimation of the direct (visible) production losses such as milk loss, mortality loss, and draft power loss, and indirect impacts like control costs (Knight-Jones and Rushton, 2013) using the method described in Jemberu et al. (2014). However, due to information paucity, impacts of the other direct losses due to reduced bodyweight, abortion, infertility, culling, and poorer hide quality were not considered in this study. Only affected herds were included in the calculations. All costs are expressed as median costs as the distribution is not Normal.

\subsubsection{Mortality loss}

The mortality loss was set equal to the market value of the animal that died. Thus, the economic loss due to mortality per herd was calculated by considering the seven categories of animals (calf, bull, heifer, dry cow, pregnant cow, lactating cow, and ox) that died and their corresponding market price (Formula 1).

$$
M L S D_{i}=\sum_{j=1}^{7} N M C_{i j} * P C_{i j}
$$

Where MLSDi represents the economic losses due to LSD induced death of herd $\mathrm{i}$; NMCij is the number of animals that died in each category $j$ of herd $i$ and PCij is the price of that animal.

\subsubsection{Milk loss}

LSDV infections in lactating cows cause milk yield reduction or cessation of milking for the duration of the illness and sometimes beyond. The economic loss per herd due to loss of milk production was estimated based on Formula 2.

$$
\text { MilkLSD }_{i}=\text { NLSDCow }_{i} * D_{i} * \text { QMilkL }_{i} * \text { PMilk }_{i} \quad \text { [2] }
$$

where MilkLSDi represents the economic losses due to milk loss for herd i; NLSDcowi the number of LSD infected lactating cows in herd i; Di the average duration of illness in days of affected lactating cows; QMilkLi the average quantity of milk lost in litres per affected cow per day, and PMilki the price of milk per litre for herd i.

\subsubsection{Draft power loss}

In Ethiopia, the traditional agricultural system depends heavily on animal draft power to cultivate crops. A diseased draft ox cannot plough or provides less draft power. The loss from draft power reduction can be captured from effective working days lost (Formula 3). 


$$
\operatorname{DraftLSD}_{i}=\text { NoxenLSD }_{i} * \text { DDraft }_{i} * \text { PDraft }_{i} * \frac{65}{365} \quad \text { [3] }
$$

where DraftLSDi represents the economic loss due to draft power loss for herd i; NoxenLSDi the number of oxen affected in herd i, DDrafti the average duration of illness in days of an affected ox, PDrafti the price of draft power rent of an ox per day and 65/365 is an adjustment factor for effective working days - a draft ox in Ethiopia works for about 65 days in a year (Goe, 1987). Farmers whose draft oxen are affected with LSD have to rent, purchase a replacement ox or borrow animals for cultivation. An ox can be rented from a farmer owning surplus oxen on cash or grain basis.

\subsubsection{LSD control costs}

LSD control costs were considered to consist of vaccination, diagnosis and medication costs and extra labour costs for seeking treatment for sick animals. Many herd owners in Ethiopia use public veterinary services to get their animals vaccinated which is free of charge for contagious and transboundary animal diseases like LSD. However, clinical treatment of LSD affected animals was at the farmers' own expense. Hence, the economic cost of LSD treatment is calculated as per Formula 4.

$$
\operatorname{TrCost}_{i}=\left(\operatorname{NTr}_{i} * P \operatorname{Tr}_{i}\right)+\left(\text { NhoursL }_{i} * P d l_{i}\right)[4]
$$

where TrCosti represents the treatment cost for affected herd i; NTri the number of animals treated; PTri the average per head expenditure to LSD treatment; NhoursLi the average number of working hours lost for seeking treatment for sick animals, and Pdli the average payment rate of a replacement labourer per hour in the locality of herd i.

\subsubsection{Total economic losses}

The total economic costs (TEC) due to LSD infection per affected herd were obtained by adding losses arising from draft power loss, milk production loss, mortality and treatment expenditure (Formula 5).

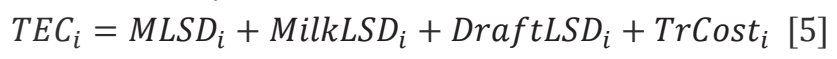

\subsubsection{Partial budget analysis for LSD vaccine use}

The cost effectiveness of LSD control through vaccination was evaluated using partial budgeting analysis technique, which quantifies the economic consequences of a specific change in farm procedures (Dijkhuizen et al., 1995). The economic concept of partial budgeting is important for cost-benefit analysis of disease control measures (Rushton, 2009). A partial budget format with four parts (additional returns gained, reduced costs, returns foregone, and extra costs experienced as a consequence of the change) was employed as described by 
Dijkhuizen et al. (1995) and Dijkhuizen and Morris (1997). Costs were estimated in scenarios with and without vaccination. The base plan was no vaccine use by the herd owners, and the alternative plan was LSD vaccine use. The cost for purchase and administration of the LSD vaccine was considered the extra cost of the alternative plan, though it is borne by the government. The profitability of vaccine use in LSD control was calculated on a herd basis using Formula 6.

Net Profit $=($ Additional returns + Reduced costs $)$

$$
\text { - (Returns foregone + Extra costs)[6] }
$$

A positive net result indicates that LSD vaccination is desirable from an economic point of view (Dijkhuizen et al., 1995; Dijkhuizen and Morris, 1997; Young et al., 2013). Moreover, the marginal rate of return (MRR) was calculated as the net benefit divided by the total cost incurred due to vaccine use to further scrutinize the adoption of the change (Gari et al., 2011).

\subsubsection{Statistical analysis}

Descriptive statistics were used to calculate the morbidity and mortality at animal and herd level. A Chi-square test was used to evaluate the differences in morbidity and mortality between categories of animals and between districts. Kruskal-Wallis equality-of-populations rank test was used, as the economic losses were not normally distributed, to compare the differences in herd level economic losses among districts and between farm types. A p-value less than 0.05 was considered as significant. Stata version 14 was used for all analyses.

\subsection{Results}

\subsubsection{Herd size and structure}

A total of 243 herds with 4430 heads enrolled in the study. The study population comprised $18.4 \%$ calves, $22.7 \%$ heifers, $8.9 \%$ bulls, $37.1 \%$ cows and $12.9 \%$ oxen. Herd size varied from $1(n=3)$ to $643(n=1)$ animals. About $90 \%$ of the herds consisted of less than 25 animals. The mean herd size in commercial farms was 56 heads and 10 heads in the subsistence farms. The majority of the farms (81.9\%) involved in the study were small holder subsistence farms, but they hold only $44.3 \%$ of the study animals; $78.6 \%$ of the herds were managed extensively.

\subsubsection{LSD morbidity and mortality}

All herd owners approached were able to describe LSD in terms of its key epidemiologic features and symptoms. Based on the farmer's response, a total of 941 out of 4430 (21.2\%) animals and 200 out of 243 (82.3\%) herds were declared affected by LSD (i.e. they had at least one LSD positive animal) in the period May 
2014 to April 2015. Mortalities at animal and herd level were 4.5\% (198/4430) and $24.3 \%$ (59/243), respectively. Case fatality amounted to $21.0 \%(198 / 941)$. In most herds in which animals died it was restricted to 1 ( $n=36$ out of 59) or 2 (9 out of 59) dead animals, however in one large herd (331 heads) 40 animals died. Differences in morbidity and mortality between study districts, at both animal level and herd level, were statistically significant $(P<0.05)$. The highest animal level morbidity $(37.9 \%)$ and mortality (12.1\%) were recorded in Jabitenan district and Selale dairy Dev. PLC, respectively (Table 6.1). The morbidity per animal category varied from lowest $15.0 \%$ in dry cows to $26.9 \%$ in oxen, whereas the mortality varied from $2.2 \%$ in dry cows to $6.0 \%$ in pregnant cows (Table 6.2). The difference in animal level morbidity and mortality between categories was significant $(P<0.05)$.

\subsubsection{Perception of herd owners on LSD impact}

From 243 herd owners interviewed in this study, 229 (94.2\%) ranked LSD as a serious or very serious disease. Economic losses most frequently mentioned were death, milk loss, draft power loss, weight loss, abortion and hide quality loss (Figure 6.2). 224 (92.2\%) of the herd owners indicated that LSD outbreaks affect cattle marketing. A large proportion ( $n=217,89.3 \%$ ) of them witnessed that cattle selling is practiced during LSD outbreaks. Almost all herd owners do not sell sick animals and $32(13.2 \%)$ of them would like to sell unaffected animals from their herds during LSD outbreaks mainly due to fear of the disease $(n=30,93.8 \%)$.

\subsubsection{Financial losses of LSD outbreaks}

The financial losses related to mortality, milk reduction, draft power loss, and control cost per affected individual animal are presented in Appendixes 6.1-6.4, respectively. The overall median financial loss per dead animal was estimated at USD 375; however, it was USD 325 for local Zebu and USD 1250 for Holstein-Friesian local Zebu cross cattle. Category wise, the median loss per head varied from USD 150 for calves to USD 1181 for milking cows, whereas from breed perspective the highest loss (USD 2250) was recorded in cross breed cows and the lowest (USD 59) in local Zebu calves. District wise, the median loss per dead animal varied from USD 125 in local Zebu in Debrelibanos district to USD 1966 in cross breed cattle in Holeta (Appendix 6.1). Besides to the mortality loss, additional costs were incurred for carcass disposal. For this a cost of USD 11.9 (ranging USD 5-20) per carcass was required, but this was not included in the economic loss estimation due to the fact that expenditure for this purpose is required in rare occasions as usually the carcasses are disposed or buried by the villagers. 


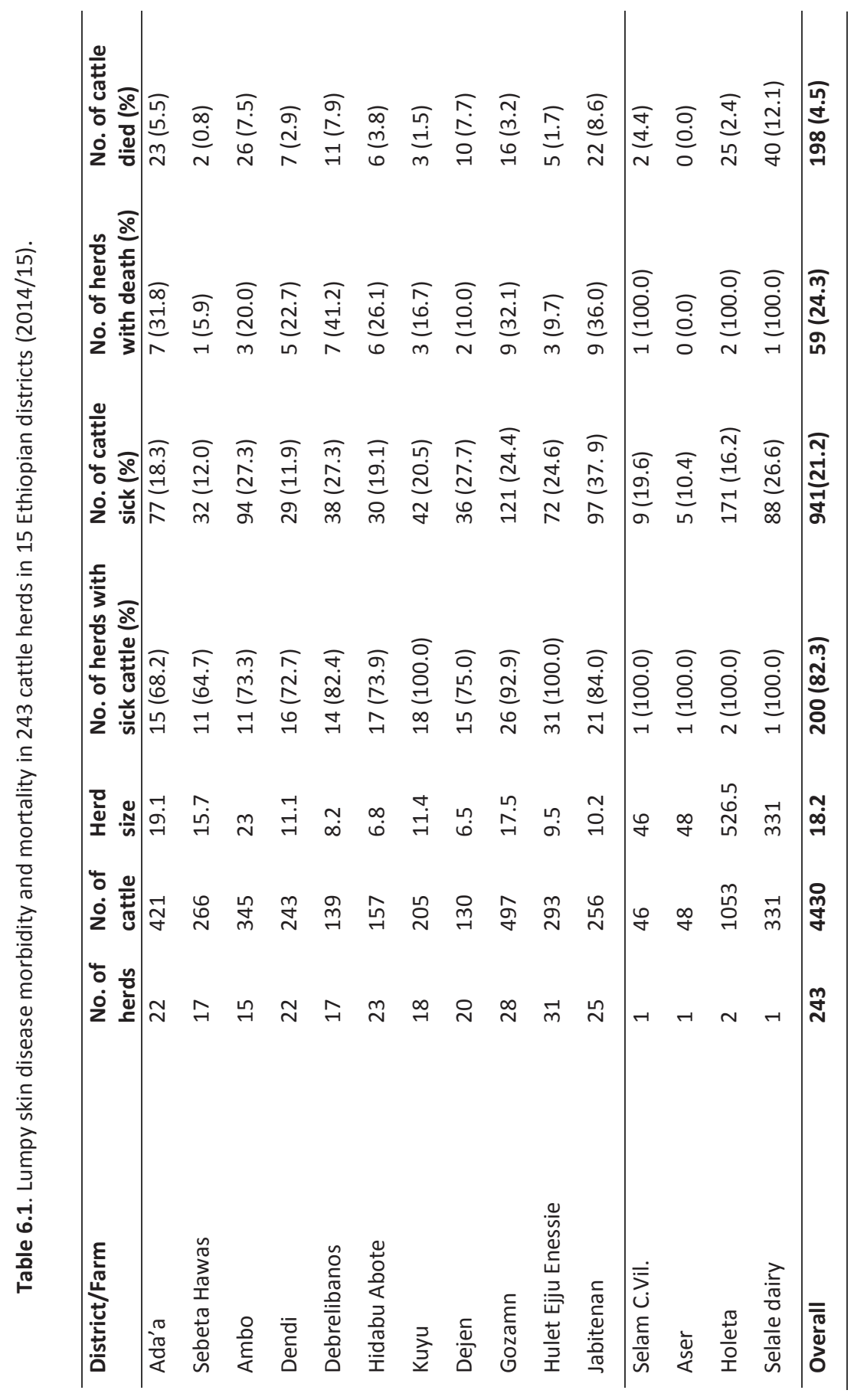




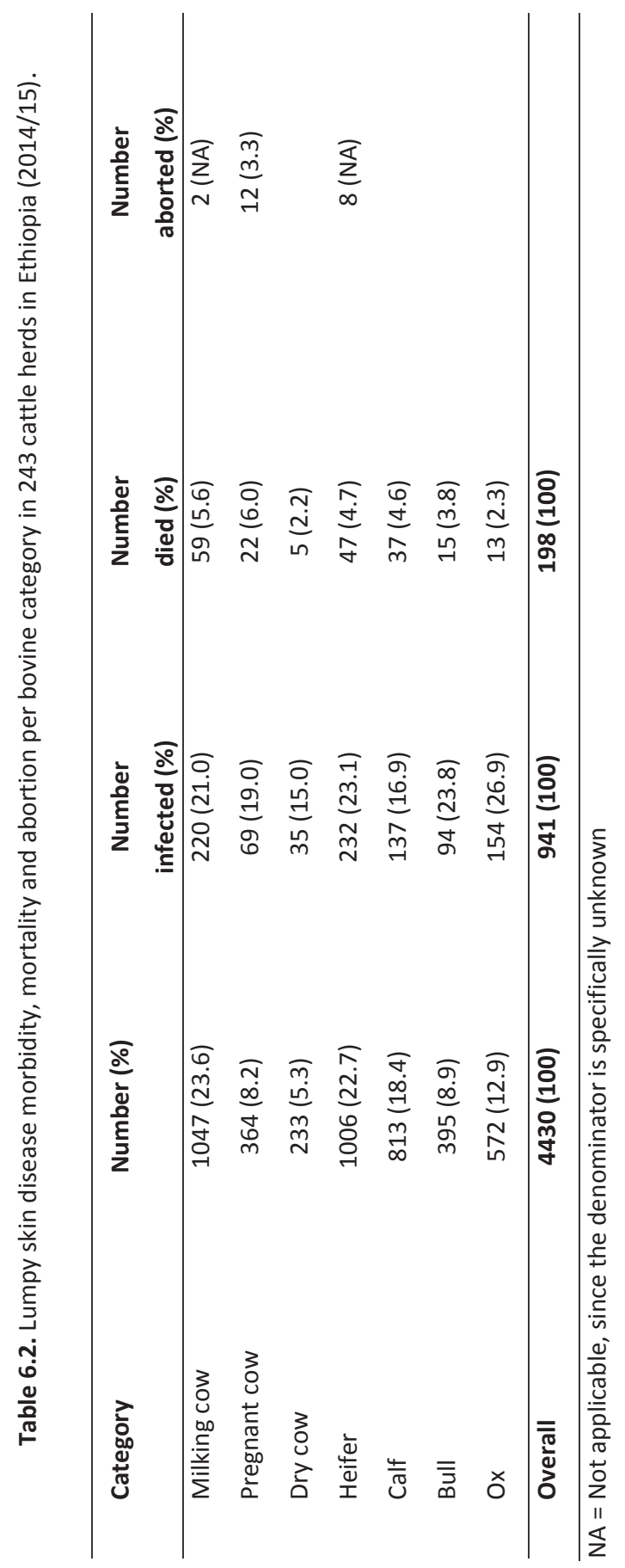


Almost all ( $n=240,98.8 \%$ ) of the herd owners knew the effect of LSD on milk production. According to the information obtained from the herd owners, milk production reduced by $74 \%$ for a period of about 2.5 months. The overall daily milk loss per affected milking cow was 4.0 litres. Breed wise, it was 1.7 litres in local and 7.2 litres in cross bred cows. Financially, the overall median milk production loss per affected milking cow was USD 141, which was USD 63 in local Zebu cow and USD 216 in Holstein-Friesian local cross cow. The lowest and the highest milk loss per milking cow reported were USD 27 in local cattle and USD 906 in cross cow in Hulet Ejju Enessie and Debrelibanos districts, respectively (Appendix 6.2).

Almost all ( $n=241,99.2 \%$ ) interviewees responded that LSD affects the traction power of animals. The median number of effective working days lost per affected ox was 10 days (range 1-32 days) resulting in an overall median loss of USD 36 per affected ox (Appendix 6.3).

More than $80 \%$ of LSD affected cattle got treated for secondary complications. The overall median diagnosis and medication cost per affected animal was USD 5 (Appendix 6.4). The cost of time lost for seeking treatment per affected animal could not be estimated as it was common practice that a herd owner took several animals to a veterinary clinic at a time to seek treatment and this complicated the estimation of per head cost.

The median total economic loss of an LSD outbreak at herd level was USD 1176. This figure is based on 193 herds as in 7 herds the LSD positive animal(s) were not productive and were not treated. A statistical analysis with Kruskal-Wallis equality-of-populations rank test revealed significant differences $(P<0.05)$ in total economic loss among districts. The highest and lowest economic losses were recorded in Selale dairy farm and in Sebeta Hawas district, respectively (Table 6.3). At herd level, the largest component of the economic loss was due to mortality (USD 1000) followed by milk loss (USD 120) and draft loss (USD 48). LSD control costs were the least contributor to herd level losses (Table 6.3). The median economic loss by farm type was USD 489 and USD 2735 in subsistence and commercial farms respectively per affected herd $(P<0.05$; Table 6.3). 


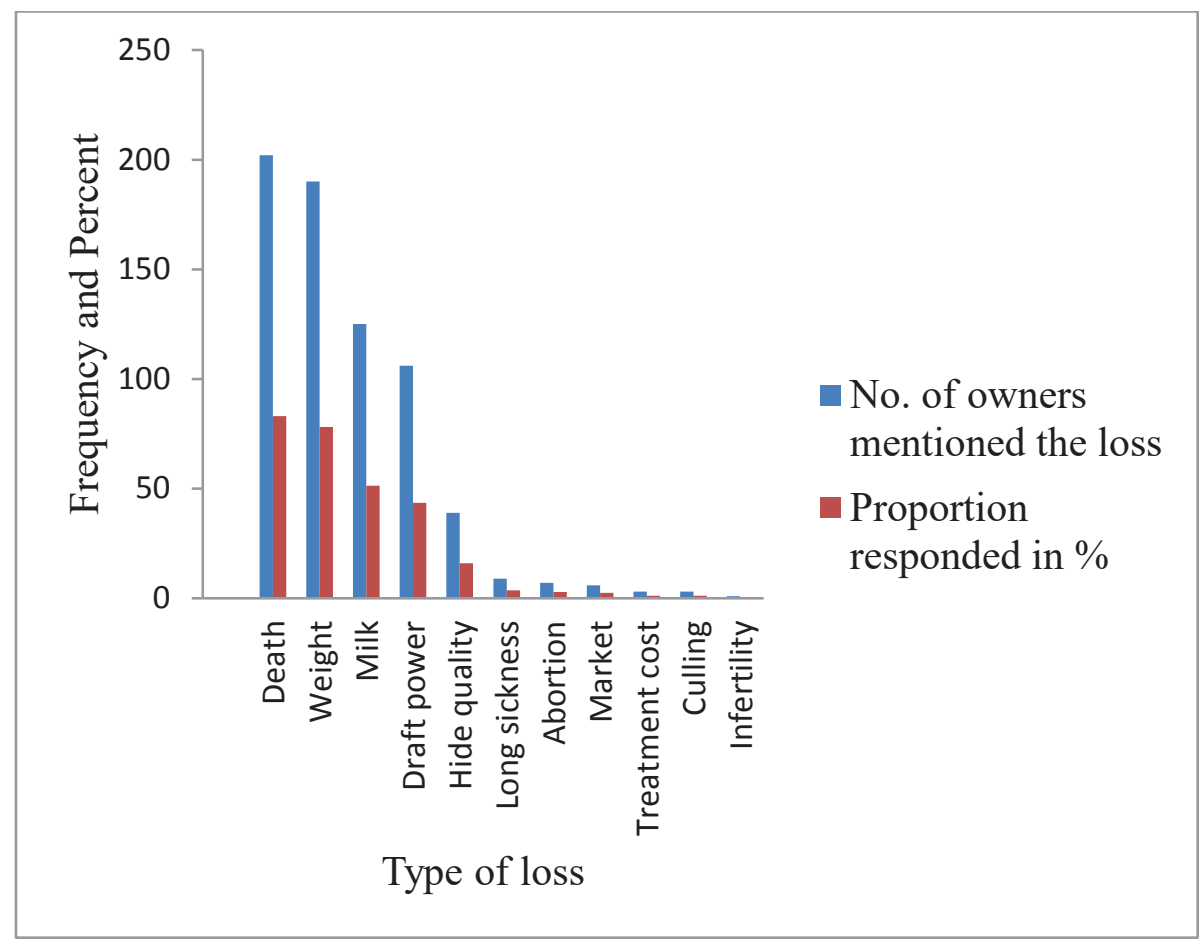

Figure 6.2. Major losses induced by lumpy skin disease as listed by cattle herd owners ( $\mathrm{n}$ $=243)$ in Ethiopia (2014/15).

\subsubsection{Partial budgeting}

The majority of the input parameters for the partial budget analysis were obtained from data collected in this study; however, the remaining key parameters were taken from other sources (Appendix 6.5).

The results of the partial budget analysis indicated a positive net profit of USD 136 (USD 56 for subsistence farm herds and USD 283 for commercial herds) and marginal rate of return (MRR) of 15.14 (11.29 in subsistence and 10.10 in commercial herd) per herd by vaccinating the animals for LSD (Table 6.4). Thus, investment in vaccination to control LSD would reduce the overall financial loss due to the disease by $11.6 \%$ per herd. 


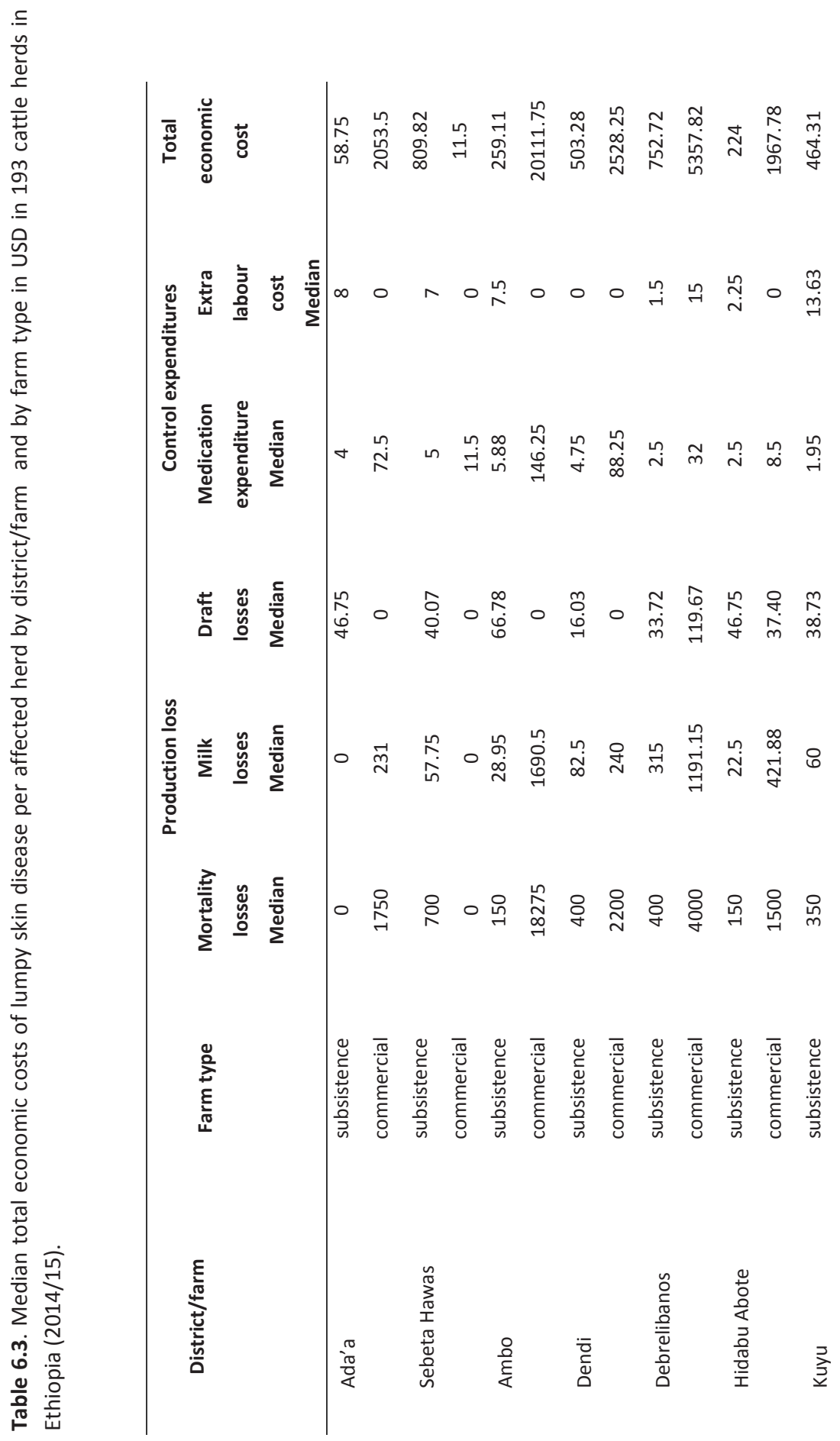




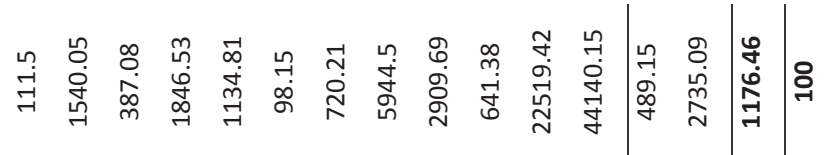

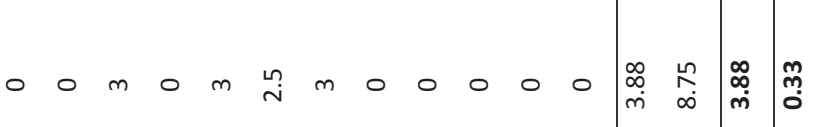

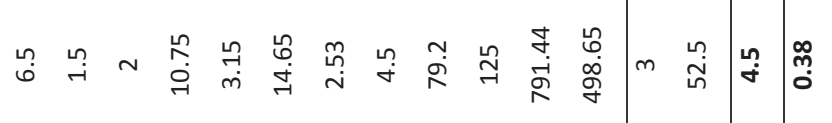

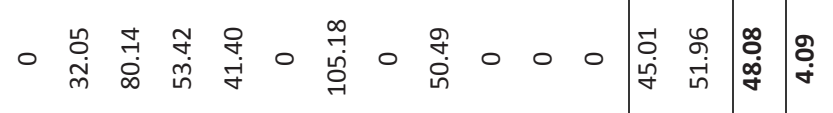

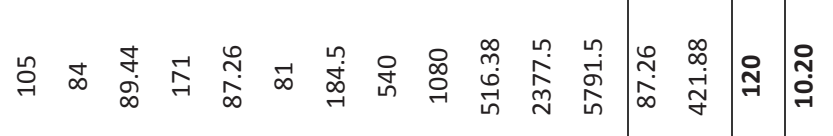

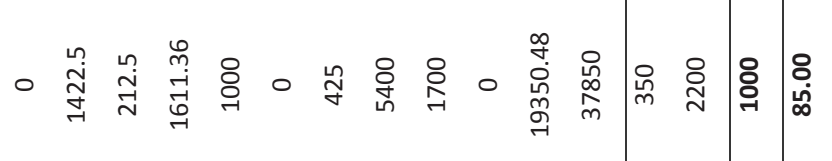

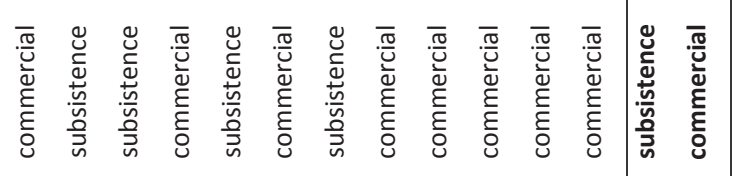

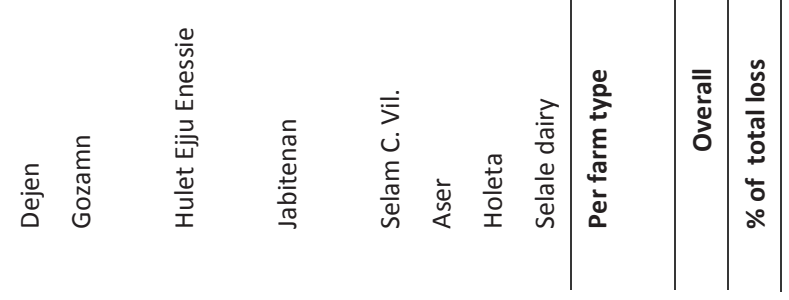




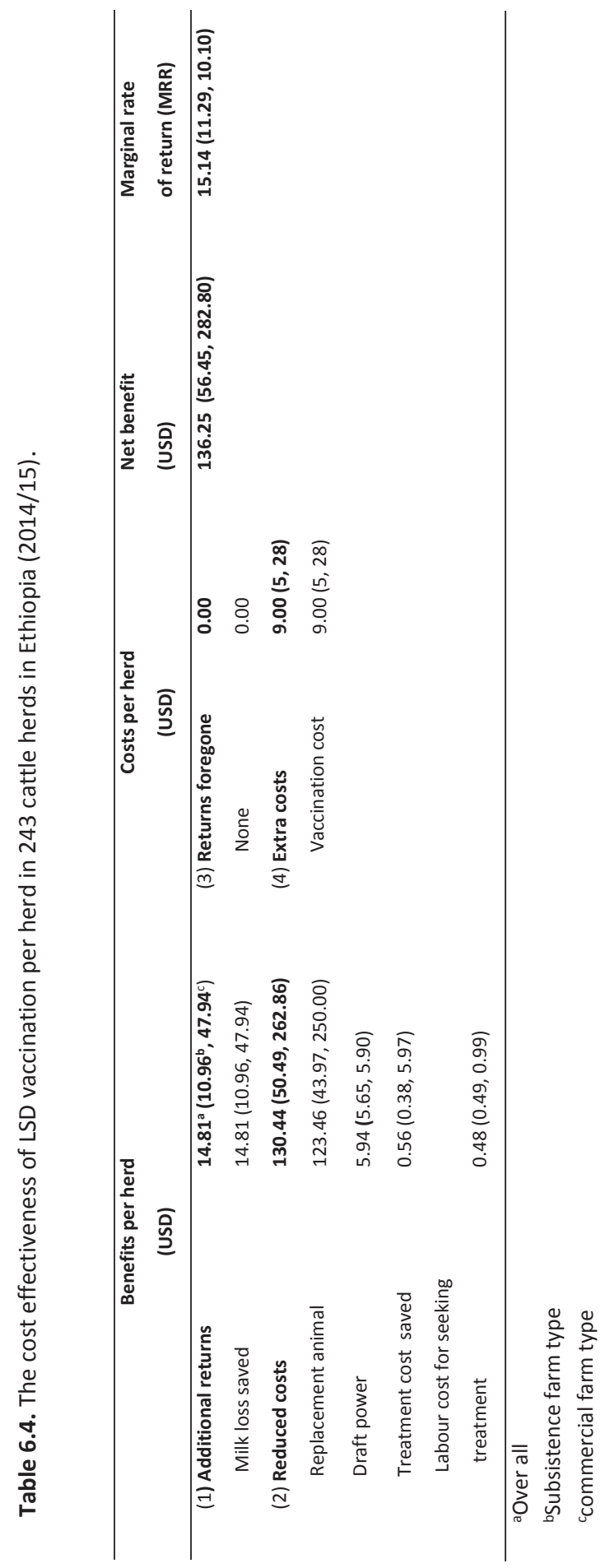




\subsection{Discussion}

The animal level morbidity (21.2\%) and mortality (4.5\%) recorded in this study is close to the $22.9 \%$ and $26 \%$ morbidity and 2.3 and $1.9 \%$ mortality reported in central Ethiopia (Ayelet et al., 2013) and Jordan (Abutarbush et al., 2015), respectively. However, it is much higher than the $7.4 \%$ animal level morbidity reported in north-eastern Ethiopia (Hailu et al., 2014), 8.7\% in Greece (Tasioudi et al., 2016), 11\% in Israel (Brenner et al., 2009), and 0.65\% in Turkey (Ince et al., 20016 ). Significantly different morbidity and mortality was observed between animal categories with oxen showing the highest level of morbidity (26.9\%). This might be attributable to the stress and fatigue created during ploughing. The highest mortality was observed in pregnant cows (6\%) which might be related to physiological conditions of pregnancy that make the animal more susceptible to disease (Kehrli et al., 2009). Generally, LSD morbidity varies from as low as $5 \%$ to $100 \%$ (Woods, 1988) and mortality is generally low (usually less than $5 \%$ ) but may sometimes reach $20 \%$ (Woods, 1988; OIE, 2010). Thus, the animal level as well as the LSD morbidity and mortality levels per animal category reported in this study are within the limits reported in previous works. Furthermore, a significantly different morbidity and mortality was present between districts with highest morbidity in Jabitenan district (37.9\%). This might be related to the presence of many rivers, irrigated areas and higher temperature, making the conditions in the district suitable for the replication of arthropods and propagation of LSD (Davies, 1991).

Interview results indicated that LSD is a serious problem for cattle producers in the study area as more than $94 \%$ of the interviewees considered LSD as a threat for their cattle. According to the herd owners, the disease induces weight loss, reduced milk production, draft power loss, mortality, market instability, infertility, abortion, culling, and hides quality losses. These observations are in line with the impacts of LSD described in previous works (Woods, 1988; Davies, 1991; Kumar, 2011; Abutarbush et al., 2015). The impacts of LSD in domestic as well as international cattle market is complex and generally go beyond the immediate effects on affected producers (Otte et al., 2004). In this study, more than $92 \%$ of the herd owners reported that LSD outbreaks affects cattle marketing at domestic market in numerous ways including lowering the demand and price of cattle during the outbreak period.

An overall median financial loss of USD 375 per dead animal recorded in this study is a big loss for a farmer whose livelihood depends on crop-livestock or livestock production. The mortality loss per head was highly variable between breeds, animal categories and districts. The per head mortality loss of local Zebu 
cattle was low (USD 325) as compared to Holstein-Friesian local cross cattle (USD 1250). The median loss per head categories varied from USD 150 for calves to USD 1181 for milking cows. These differences can be mainly attributed to the high production potential of cross bred animals and animal's purpose.

The milk production loss of $74 \%$ for the period of about 2.5 months recorded in this study is almost comparable to what has been reported in previous studies (Woods, 1988; Kumar, 2011; Abutarbush et al., 2015). The median daily milk loss of 4.0 litres per affected animal is a big loss for a nation that is an importer of dairy products (Negassa et al., 2011) by aggravating the product scarcity. In most cases the affected milking cows did not produce milk for months. For cows restarting milk production, it took months to regain their normal production level while in some cases, especially for local cows, LSD caused complete drying off. LSD caused an overall median loss of USD 141 per affected cow, being USD 216 in Holstein-Friesian local cross and reduced to USD 63 in local Zebu. The loss indicated here is greater than the loss induced by foot and mouth disease (FMD), which was USD 29 per affected cow in crop-livestock production system and USD 26 in pastoral system (Jemberu et al., 2014).

In the current study the herd owners reported that LSD affected draft animals were not available for field work for an average period of 59 days (ranging 7-180 days) which resulted in a median loss of about 10 (ranging 1-32) effective working days. The lost working days, in turn, lead to reduced crop production, either through reduced area that can be cultivated, or through lower yields due to late planting (McDermott et al., 1999). The effective working days lost estimated in this study is smaller than the 16 days reported by Gari et al. (2011). A farmer whose ox is affected by LSD has to borrow, rent, or purchase replacement ox or request assistance from relatives for cultivation. The translation of the effective working days lost into financial loss by considering the daily renting price (cash basis) of an ox gave an overall median loss of USD 36 per affected ox, which is greater than the loss reported due to FMD (Jemberu et al., 2014). This loss would have been larger if we had used 100/365 as adjustment factor (Yilma et al., 2011) instead of 65/365.

The median total economic loss of USD 1176 per LSD affected herd recorded in this study is a huge loss for a producer in a country with a gross domestic product per capita of USD 316 (Trading-Economics, 2015) and per capita income of USD 550 (World-Bank, 2015). Even the median loss per affected herd in subsistence croplivestock system (USD 489) is six times higher than what Jemberu et al. (2014) reported for FMD, a disease which is on the top list for its devastating economic impact worldwide (Knight-Jones and Rushton, 2013; Junker et al., 2009). This supports the reports stating that LSD is economically more important than FMD in 
some countries such as South Africa (Murphy et al., 1999). The reason for this is that mortality in FMD is low and it occurs mainly in young age categories while LSD mortality is relatively high compared to FMD and occurs in all age categories. Of all costs, $85 \%$ is due to mortality although LSD induced mortality is low in cattle population as a whole (Woods, 1988). The median total economic losses per affected herd of USD 2735 for the commercial farm were significantly higher than the loss of USD 489 for the subsistence farm type. The higher loss in affected commercial herds is the reflection of larger herd size, higher market value and productivity potential of cross-bred animals.

As the study is undertaken retrospectively after certain months of LSD occurrence in the herd, recall bias in relation to the duration of infection, the amount of milk produced during sickness, working days lost and others might happened. Furthermore, the number of animals and herds affected were reported based on the owners declaration and this might also lead to biased number of cases. The recall bias and the diagnosis bias might have influenced the estimation of the financial losses reported to some extent and can be taken as the weakness of the study.

Routine vaccination, stamping-out and movement restriction are important methods in LSD control (Davies, 1991; Carn, 1993). Each control measure acts by reducing the transmission of the agent in the population. However, Ethiopia is applying mainly vaccination to control the disease. The economic benefit gained from controlling LSD with vaccination was measured by taking the reduction in economic loss from the disease into account by comparison with the level of expenditure for its vaccination. The result of the cost benefit analysis showed that a net loss of about USD 136 per herd would be avoided and marginal rate of return (MMR) of 15.14 gained by using LSD vaccination. The estimates revealed that LSD control with vaccination is economically beneficial by reducing the loss by $11.6 \%$ per herd. This result is less cost effective as compared to the findings of Gari et al. (2011) who reported a positive net benefit of USD 680.71 and a MRR of 34 for LSD vaccine intervention. However, the existing LSD vaccine provides incomplete protection against the disease (Ayelet et al., 2013). The vaccine is efficacious in only $28 \%$ of the vaccinated animals (unpublished data) which was taken into account in the partial budget analysis. More effective vaccines are needed to gain more from the intervention. The partial budget analysis was restricted to the direct benefits arising from the mortality and morbidity losses avoided and savings in the cost of LSD treatment. We did not consider other control options like movement control due to their practical limitation in Ethiopian situations.

It should be noted that only the noticeable direct costs and treatment costs associated with the disease were considered in the study. The indirect impacts of the 
disease such as under exploitation of the animal potential, animal welfare, international trade etc., were not considered. Also the visible direct costs were not fully captured mainly due to information paucity and difficulty to measure the loss in precise economic terms. Thus, the economic loss estimation presented here should be seen as a conservative estimate of the loss due to LSD.

\subsection{Conclusion}

The LSD impact in terms of production losses and control costs was high, a median total economic loss of USD 1176 (USD 2735 in commercial and USD 489 in subsistence herd) per LSD affected herd. The losses were mainly from morbidity and mortality of cattle and were the greatest in highly productive animals. The largest component of the economic losses was due to mortality loss followed by milk loss and draft loss at both animal level and herd level losses. LSD control costs were the least contributor for the herd level losses. Commercial farms which hold more productive and more susceptible animals were more severely affected economically than the subsistence crop related farms. Vaccination was found to be economically and practically feasible choice to control LSD. The cost benefit analysis was restricted to the direct benefits arising from the mortality and morbidity losses avoided and savings in the cost of LSD treatment. Generally, vaccination is economically beneficial and should be encouraged.

\section{Acknowledgments}

The authors would like to thank NUFFIC (Netherlands Organization for International Cooperation in Higher Education) for funding this project. We are grateful to the National Animal Health Diagnostic and Investigation Centre, Federal Animal Health Directorate, and National Veterinary Institute, Ethiopia for assistance in field work of the study. We also thank district animal health professionals and the herd owners for their kind collaboration in collecting and providing information for the study.

\section{References}

Abutarbush, S.M., Ababneh, M.M., Al Zoubi, I.G., Al Sheyab, O.M., Al Zoubi, M.G., Alekish, M.O., Al Gharabat, R.J., 2015. Lumpy skin disease in Jordan: disease emergence, clinical signs, complications and preliminary-associated economic losses. Transbound. Emerg. Dis. 62, 549-554.

APHRD, 2012. Ethiopia Animal Health Yearbook 2011, Animal and Plant Health Regulatory Directorate (APHRD), Addis Ababa, Ethiopia. 
AU-IBAR, 2013. Lumpy skin disease. In: (AU-IBAR), A.U.-I.B.f.A.R. (Ed.) CAB International.

Ayelet, G., Abate, Y., Sisay, T., Nigussie, H., Gelaye, E., Jemberie, S., Asmare, K., 2013. Lumpy skin disease: preliminary vaccine efficacy assessment and overview on outbreak impact in dairy cattle at Debre Zeit, central Ethiopia. Antivir. Res. 98, 261-265.

Babiuk, S., Bowden, T.R., Boyle, D.B., Wallace, D.B., Kitching, R.P., 2008. Capripoxviruses: an emerging worldwide threat to sheep, goats and cattle. Transbound. Emerg. Dis. 55, 263-272.

Brenner, J., Bellaiche, M., Gross, E., Elad, D., Oved, Z., Haimovitz, M., Wasserman, A., Friedgut, O., Stram, Y., Bumbarov, V., Yadin, H., 2009. Appearance of skin lesions in cattle populations vaccinated against lumpy skin disease: statutory challenge. Vaccine 27, 1500-1503.

Carn, 1993. Control of capripoxvirus infections. Vaccine 11, 1275-1279.

Chenais, E., Boqvist, S., Emanuelson, U., von Bromssen, C., Ouma, E., Aliro, T., Masembe, C., Stahl, K., Sternberg-Lewerin, S., 2017. Quantitative assessment of social and economic impact of African swine fever outbreaks in northern Uganda. Prev. Vet. Med. 144, 134-148.

CSA, 2015. Agricultural Sample Survey, 2014/15 (2007 E.C.), Volume II: Report on livestock and livestock characteristics (Private peasant holdings). Statistical Bulletin 578. Central Statistical Agency (CSA), Federal Democratic Republic of Ethiopia, Addis Ababa.

Davies, F.G., 1991. Lumpy skin disease, an African capripox virus disease of cattle. Br. Vet. J. 147, 489-503.

Dijkhuizen, A.A., Huirne, R.B.M., Jalvingh, A.W., 1995. Economic analysis of animal diseases and their control. Prev. Vet. Med. 25, 135-149.

Dijkhuizen, A.A., Morris, R.S., 1997. Animal Health Economics: Principles and Applications. Post Graduate Foundation in Veterinary Science University of Sydney, Sydney South 1235, Australia.

FAO, 2010. Case definition of livestock disease. ttp://www.fao.org/docrep/014/al859e/al859e00.pdf. Accessed 31 July 2017.

Gari, G., Bonnet, P., Roger, F., Waret-Szkuta, A., 2011. Epidemiological aspects and financial impact of lumpy skin disease in Ethiopia. Prev. Vet. Med. 102, 274283.

Gari, G., Grosbois, V., Waret-Szkuta, A., Babiuk, S., Jacquiet, P., Roger, F., 2012. Lumpy skin disease in Ethiopia: seroprevalence study across different agroclimate zones. Acta Trop. 123, 101-106. 
Gari, G., Waret-Szkuta, A., Grosbois, V., Jacquiet, P., Roger, F., 2010. Risk factors associated with observed clinical lumpy skin disease in Ethiopia. Epidemiol. Infect. 138, 1657-1666.

GebreMariam, S., Amare, S., Baker, D., Solomon, A., 2010. Diagnostic study of live cattle and beef production and marketing: Constraints and opportunities for enhancing the system. http://bdsknowledge.org/dyn/bds/docs/800/Ethiopia-livestock-valuechain-diagnostic-july-201.pdf. Accessessed 15 February 2017.

Goe, M.R., 1987. Animal traction on smallholder farms in the Ethiopian highlands. Department of Animal Science. Cornell University, Ithaca, New York.

Hailu, B., Tolosa, T., Gari, G., Teklue, T., Beyene, B., 2014. Estimated prevalence and risk factors associated with clinical Lumpy skin disease in north-eastern Ethiopia. Prev. Vet. Med. 115, 64-68.

Horst, H.S., de Vos, C.J., Tomassen, F.H.M., Stelwagen, J., 1999. The economic evaluation of control and eradication of epidemic livestock diseases. Rev. Sci. Tech. Off. Int. Epiz. 18, 367-379.

Ince, O., B., Cakir, S., Dereli, M.A., 20016. Risk Analysis of Lumpy Skin Disease in Turkey. Indian J. Anim. Res. 50, 1013-1017.

Jemberu, W.T., Mourits, M.C., Woldehanna, T., Hogeveen, H., 2014. Economic impact of foot and mouth disease outbreaks on smallholder farmers in Ethiopia. Prev. Vet. Med. 116, 26-36.

Jibat, T., Mourits, M.C., Hogeveen, H., 2016. Incidence and economic impact of rabies in the cattle population of Ethiopia. Prev. Vet. Med. 130, 67-76.

Junker, F., Komorowska, J., Tongeren, F.V., 2009. “Impact of Animal Disease Outbreaks and Alternative Control Practices on Agricultural Markets and Trade: The case of FMD", OECD Food, Agriculture and Fisheries Working Paper.

Kehrli, M.E.J., Ridpath, J.F., Neill, J.D., 2009. Immune suppression in cattle: contributors and consequences. In, NMC 48th Annual Meeting Charlotte, North Carolina, 103-112.

Knight-Jones, T.J., Rushton, J., 2013. The economic impacts of foot and mouth disease - what are they, how big are they and where do they occur? Prev. Vet. Med. 112, 161-173.

Kumar, S.M., 2011. An outbreak of lumpy skin disease in a Holstein dairy herd in Oman: A clinical report. Asian J. Anim. Vet. Adv. 6, 851-859.

McDermott, J., Randolph, T.F., Staal, S.J., 1999. The economics of optimal health and productivity in smallholder livestock systems in developing countries. Rev. Sci. Tech. Off. Int. Epiz. 18, 399-424. 
Mebratu, G.Y., Kassa, B., Fikre, Y., Berhanu, B., 1984. Observation on the outbreak of lumpy skin disease in Ethiopia. Rev. Elev. Méd. Vét. Pays Trop. 37, 395-399.

Mengistu, A., 2003. Country pasture/forage resource profiles. http://www.fao.org/ag/agp/agpc/doc/counprof/ethiopia/ethiopia.htm. Accessed 2 September 2015.

Metaferia, F., Cherenet, T., Gelan, A., Abnet, F., Tesfay, A., Ali, J.A., Gulilat, W., 2011. A Review to Improve Estimation of Livestock Contribution to the National GDP. Ministry of Finance and Economic Development and Ministry of Agriculture, Addis Ababa, Ethiopia.

Murphy, F.A., Gibbs, E.P.J., Horzinek, M.C., Studdert, M.J., 1999. Veterinary Virology Academic Press San Diego. pp. 278-291.

NBE, 2014. National Bank annual report of 2013-2014. National Bank of Ethiopia (NBE), Addis Ababa Ethiopia.

Negassa, A., Rashid, S., Gebremedhin, B., 2011. Livestock Production and Marketing. ESSP II Working Paper 26. International Food Policy Research Institute/Ethiopia Strategy Support Program II, Addis Ababa, Ethiopia.

OIE, 2010. Manual of diagnostic tests and vaccines for terrestrial animals, vol. 2, chapter 2.4.14, Lumpy skin disease. OIE, Paris.

http://web.oie.int/eng/normes/MMANUAL/A_Index.htm. Accessed 26 February 2016.

OIE, 2015. Manual of diagnostic tests and vaccines for terrestrial animals, vol. 1, chapter 1.1.6, principles of veterinary vaccine production. OIE, Paris. http://www.oie.int/fileadmin/Home/eng/Health_standards/tahm/1.01.06 _VACCINE_PRODUCTION.pdf. Accessed 8 March 2016.

Otte, M.J., Nugent, R., McLeod, A., 2004. Transboundary animal diseases: Assessment of Socio-economic impacts and institutional response. Livestock policy discussion paper No. 9. FAO, Livestock information and policy branch, AGAL.

Oxford-Analytica, 2012. The Costs of Animal Disease. A report produced for the Federation for International Animal Health. Oxford Analytica Ltd, 1-37.

Pritchett, J., Thilmany, D., Johnson, K., 2005. Animal disease economic impacts: a survey of literature and typology of research approaches. Int. Food Agribus. Man. 8, 23-45.

Radostits, O.M., Gay, C.C., Hinchcliff, K.W., Constable, P.D., 2007. Veterinary Medicine: A Textbook of the Diseases of Cattle, Sheep, Pigs, Goats and Horses. Sounders Elsevier Spain. pp. 1424-1426.

Rushton, J., 2009. The Economics of Animal Health and Production. CABI Oxfordshire, UK. pp. 73-197. 
Tasioudi, K.E., Antoniou, S.E., Iliadou, P., Sachpatzidis, A., Plevraki, E., Agianniotaki, E.I., Fouki, C., Mangana-Vougiouka, O., Chondrokouki, E., Dile, C., 2016. Emergence of lumpy skin disease in Greece, 2015. Transbound. Emerg. Dis. 63, 260-265.

Trading-Economics, 2015. Ethiopia GDP per capita 1981-2015.

Tuppurainen, E.S., Oura, C.A., 2012. Review: lumpy skin disease: an emerging threat to Europe, the Middle East and Asia. Transbound. Emerg. Dis. 59, 40-48.

Tuppurainen, E.S., Venter, E.H., Shisler, J.L., Gari, G., Mekonnen, G.A., Juleff, N., Lyons, N.A., De Clercq, K., Upton, C., Bowden, T.R., Babiuk, S., Babiuk, L.A., 2015. Review: Capripoxvirus diseases: current status and opportunities for control. Transbound. Emerg. Dis. doi:10.1111/tbed.12444.

WAHIS, 2016. Summary of Immediate notifications and Follow-ups. World Animal Health Information System (WAHIS interface). http://www.oie.int/wahis_2/public/wahid.php/Diseaseinformation/Imms ummary. Accessed 18 July 2016.

Woods, J.A., 1988. Lumpy skin disease- A review. Trop. Anim. Hlth. Prod. 20, 11-17. World-Bank, 2015. Economic over view. World Bank.

Yilma, Z., Emannuelle, G.B., Ameha, S., 2011. A review of the Ethiopian dairy sector. Food and Agriculture Organization of the United Nations, Sub Regional Office for Eastern Africa (FAO/SFE) Addis Ababa, Ethiopia.

Young, J.R., Suon, S., Andrews, C.J., Henry, L.A., Windsor, P.A., 2013. Assessment of financial impact of foot and mouth disease on small holder cattle farmers in Southern Cambodia. Transbound. Emerg. Dis. 60, 166-174.] 


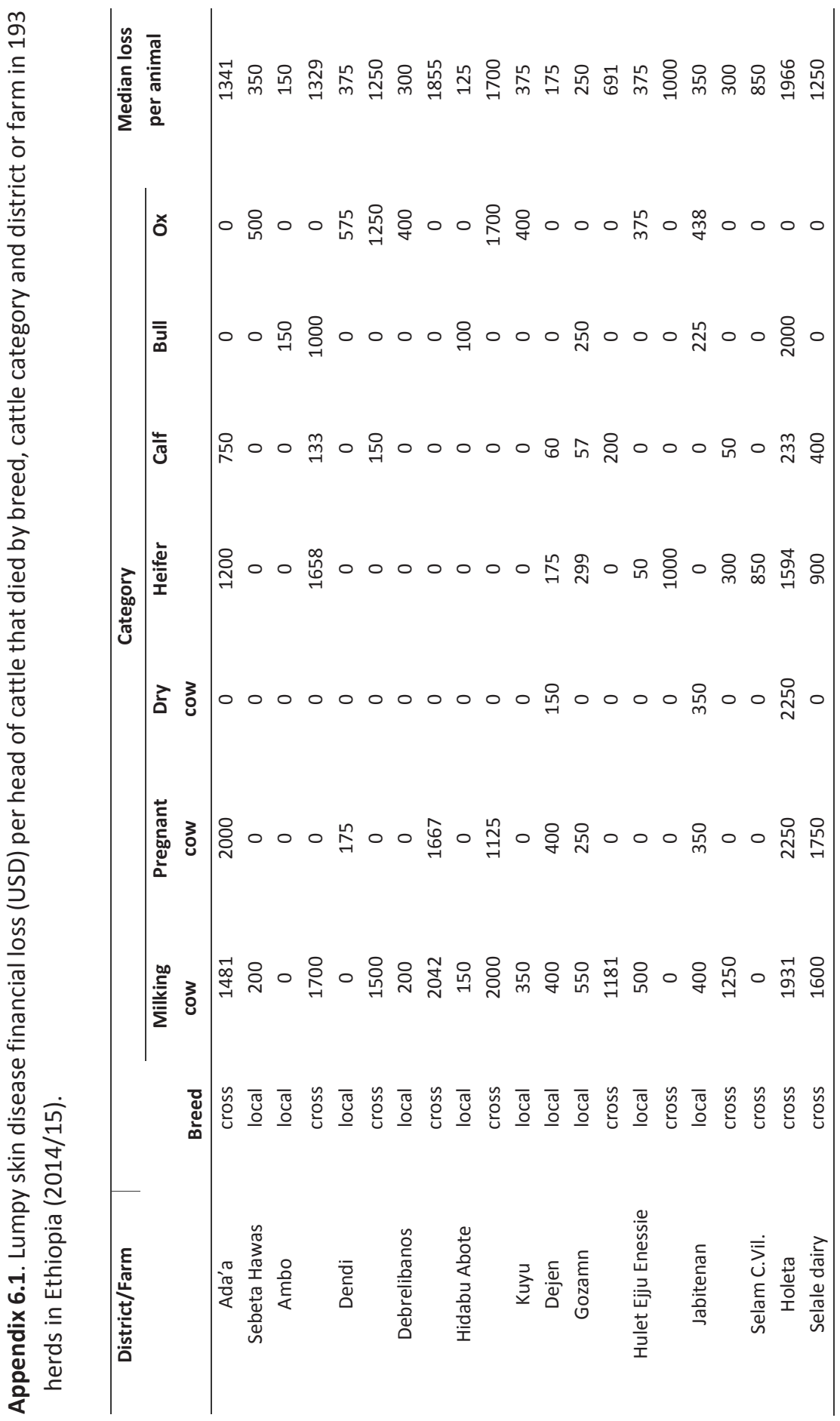




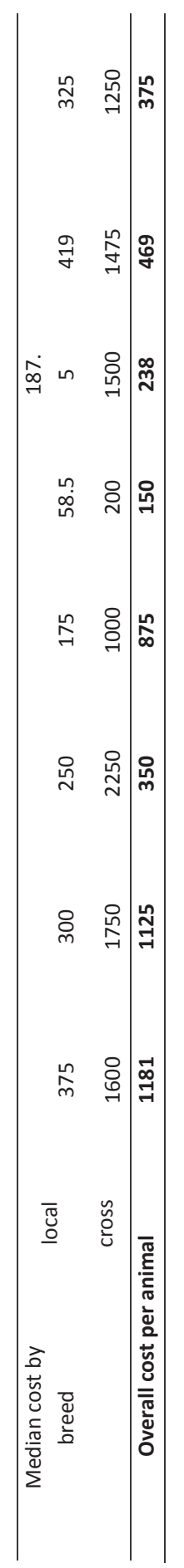




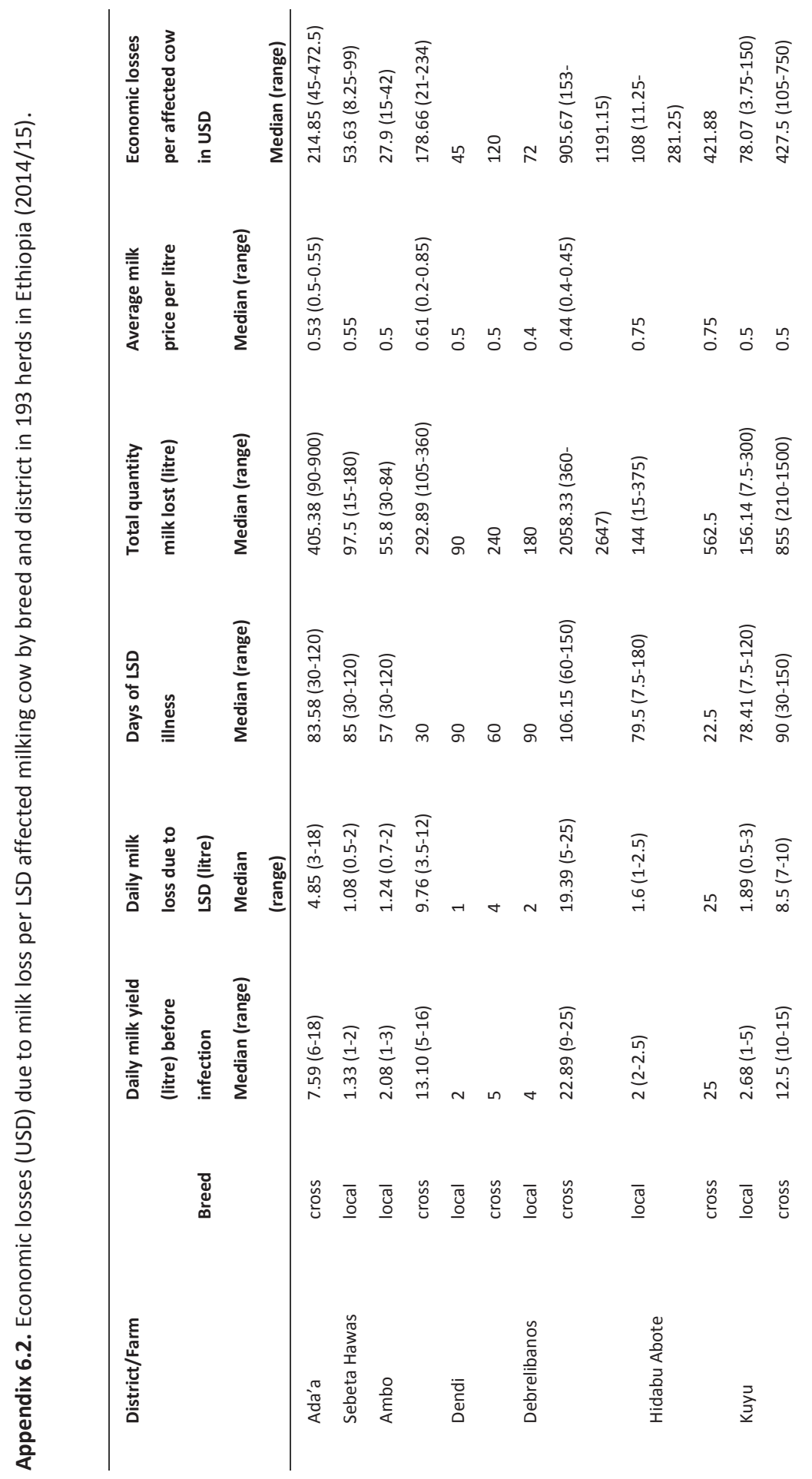




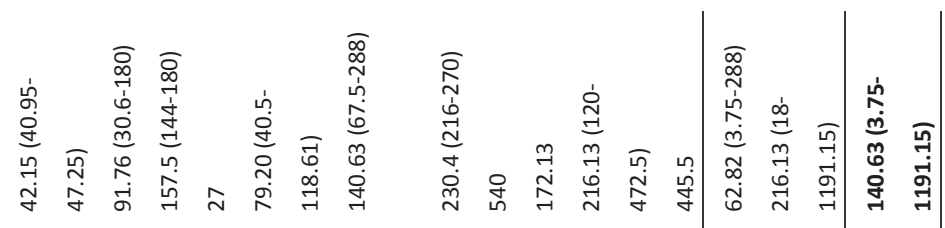

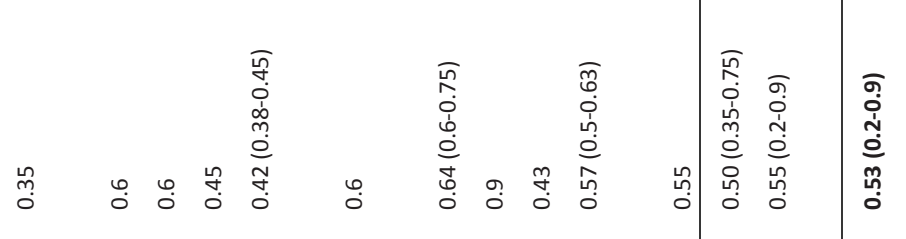

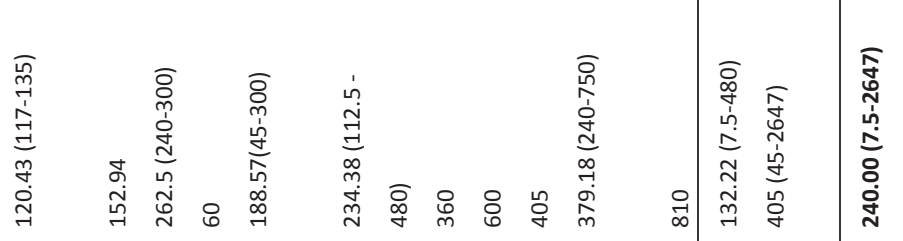

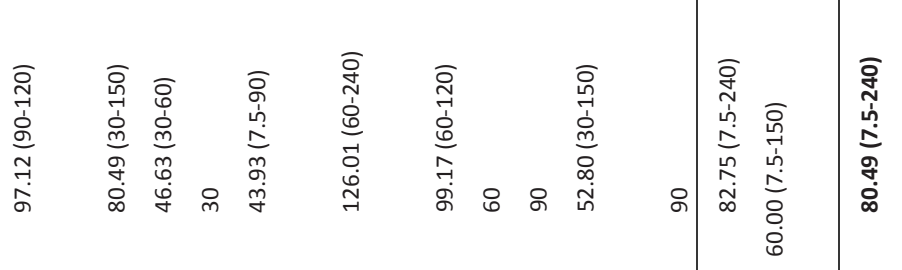

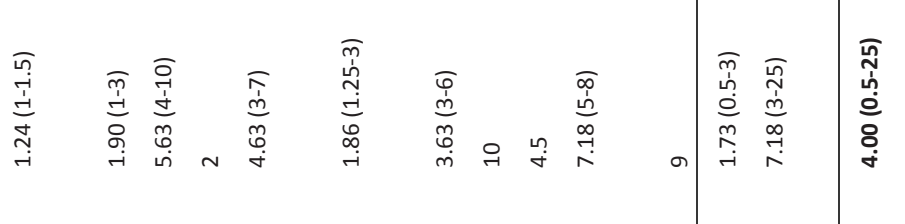

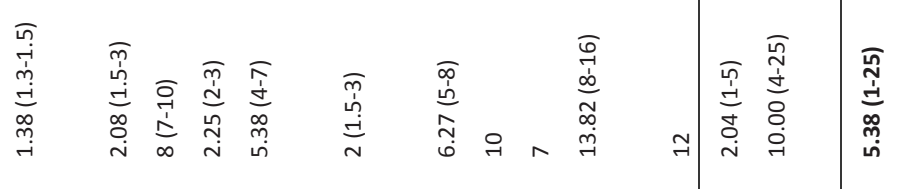

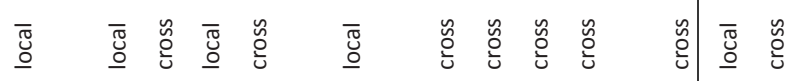

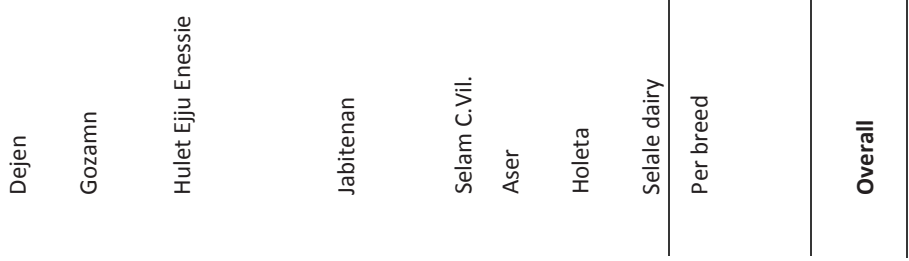




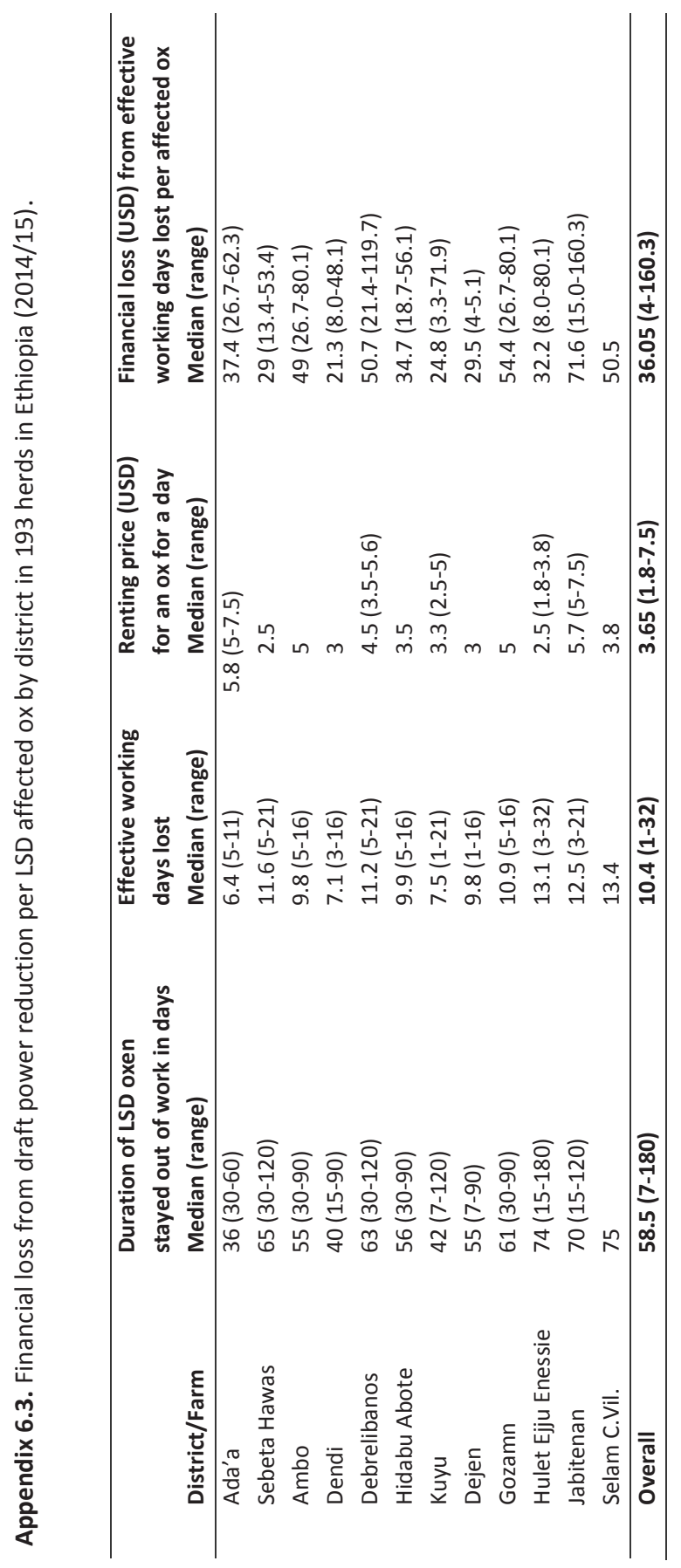




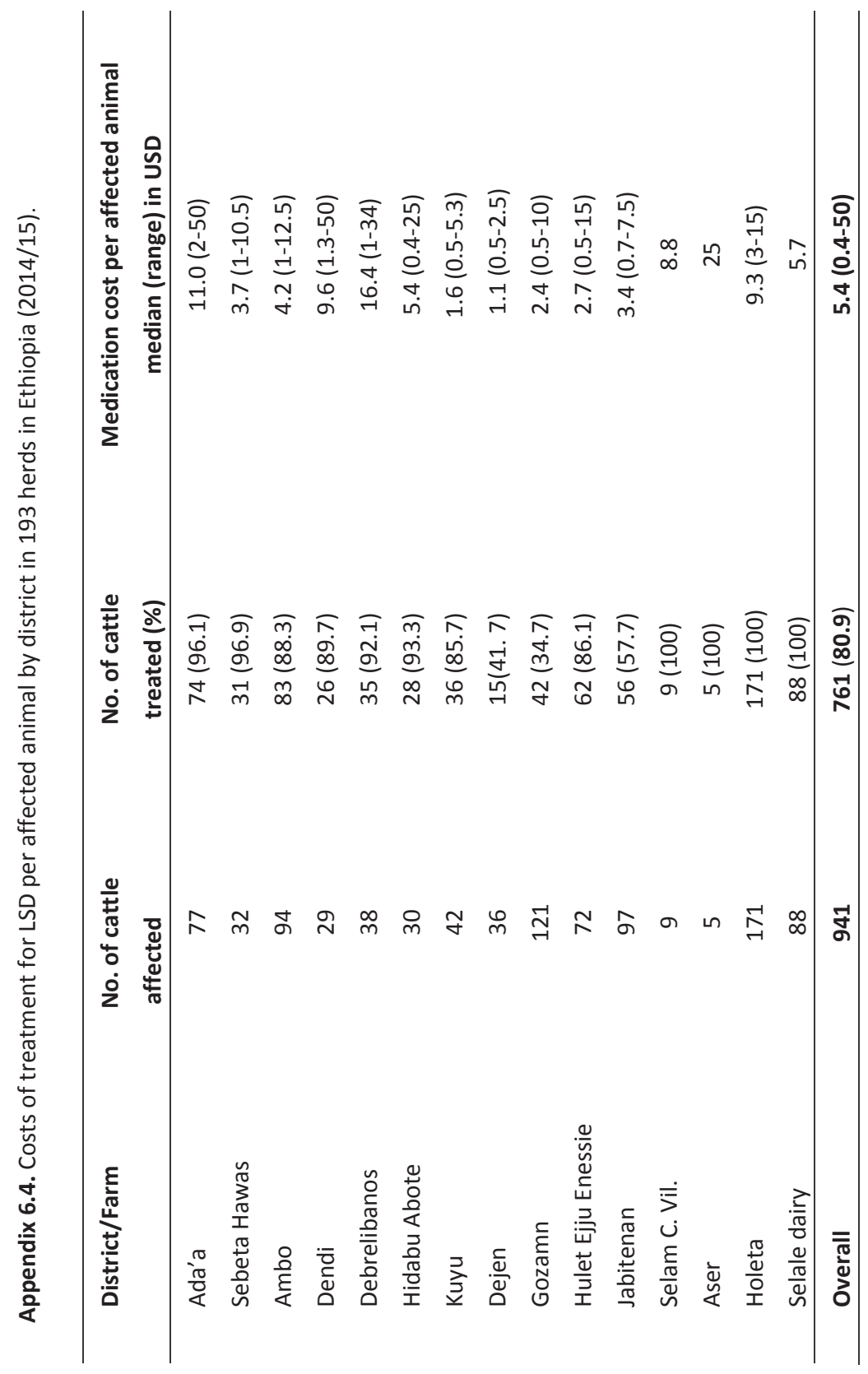




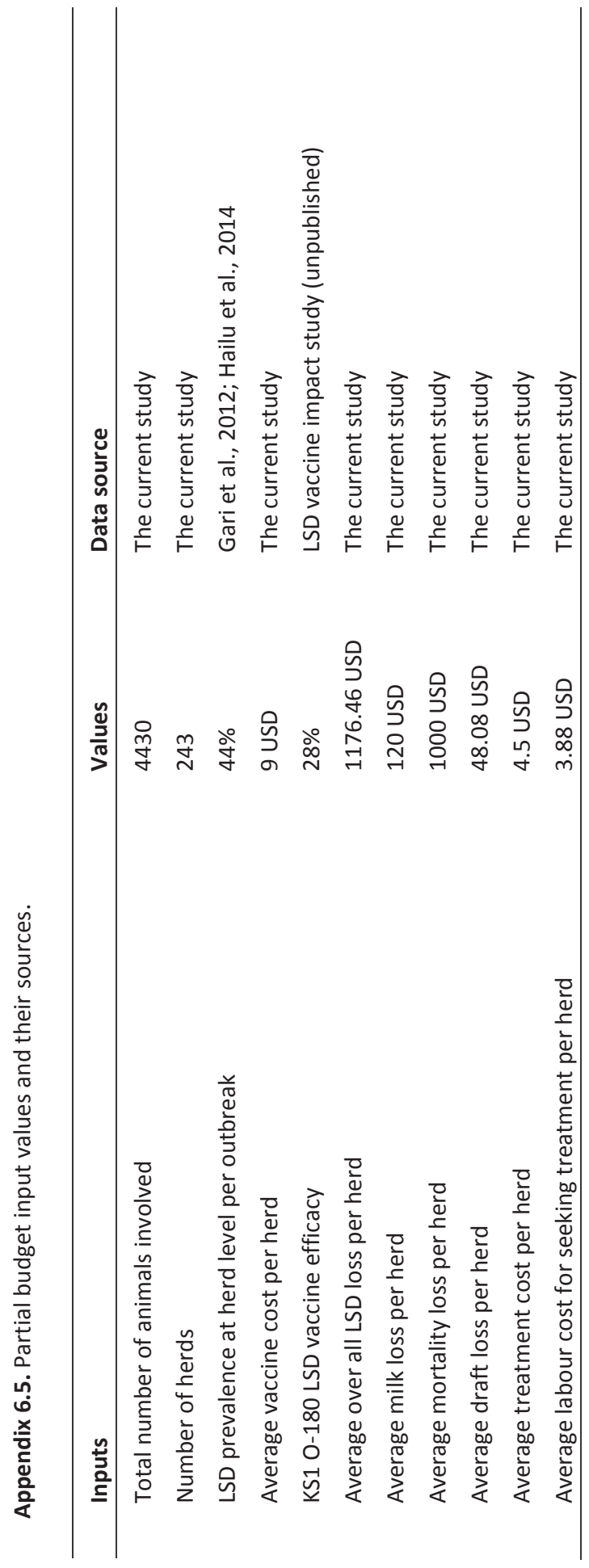




\section{7}

General discussion 



\subsection{Introduction}

The main aim of the studies described in this thesis was to generate knowledge on lumpy skin disease (epidemiology, financial consequences and effect of vaccination) in cattle that can be used to formulate and optimize a control strategy in Ethiopia. LSD is one of the top ranked diseases that affects the livelihood of many Ethiopians as approximately $70 \%$ of them, directly or indirectly, depend on the livestock sector (GebreMariam et al., 2010). In Ethiopia, cattle are kept for multiple purposes: meat and milk production, draft power, manure for fuel and fertilizer, cash storage for those that have no bank access and for social status. Farmers' livelihoods are often threatened by diseases that lead to death of their animals, reduce production and productivity or cause a drop of demands (Perry and Grace, 2009). The African Union-Inter African Bureau for Animal Resources (AU-IBAR, 2014) records indicate that LSD is the fourth most widely distributed transboundary animal disease in Africa following Newcastle disease, foot and mouth disease (FMD) and peste des petis ruminants (PPR). Hence, it is the second after FMD if only cattle diseases are considered.

The motivation for the research described in this thesis was the (ongoing) extensive LSD outbreak having a significant economic impact on the country for the past decades. The specific aims can be found in Chapter 1. In this final chapter, research findings are discussed and the practical implications of the results towards control of LSD, future research directions and the main conclusions are presented.

\section{2 Amalgamation of the findings}

\subsubsection{LSD geographical distribution and level of occurrence}

Understanding the epidemiology of a disease such as geographical distribution, its morbidity, mortality, and risk factors is very important for designing and justifying an effective control program (OIE, 2014). The epidemiological information described in Chapter 2, 3, 4 and 6 revealed the importance of LSD in Ethiopia and therefore will help to formulate a control strategy for the disease in the country.

LSD has been spreading into several parts of Ethiopia following its first introduction in the north-western part in 1981 (Mebratu et al., 1984; Gari et al., 2010). A number of reports describe the occurrence of LSD in several parts of Ethiopia (Gari et al., 2010; APHRD, 2012; Ayelet et al., 2014; Hailu et al., 2014; Abera et al., 2015; Gelaye et al., 2015). The spatiotemporal study described in Chapter 2 clearly indicates that LSD is now a well-established endemic disease in Ethiopia because all of the regional states and city administrations reported at least one LSD 
outbreak in the period 2000 to 2015. At local level, LSD occurs as an epidemic for a certain period and spreads throughout a regional state over a period of several years (Chapter 2). The prevalence study (Chapter 3) confirmed that LSD is widespread in central and north-western parts of Ethiopia, with all investigated kebeles ( $n=30$ ) having at least one LSD seropositive herd. LSD has been reported to occur in all agroclimatic zones of Ethiopia, from the hot arid lowland, warm wet midland to cool highlands (Chapter 2, Chapter 3) showing the potential of LSDV to spread in a range of climatic zones, in accordance with Gari et al. ( 2010).

Furthermore, the spatiotemporal study showed that, at district level, on average more than one seventh of the districts in the nation is stricken by LSD outbreaks every year (Chapter 2). LSD outbreak incidence varied over space with the lowest incidence (over the period 2000-2015) observed in the eastern lowland (Afar and Somali), southern lowland (Liben), south west (Benchi Maji) and North (northwestern zone of Tigray). This might be attributed to the less favourable environmental conditions for the transmission and circulation of LSDV. Most of these areas are characterized as dry (Ecotravelworld wide) which may not be conducive for the replication and survival of arthropods that are considered the major vector for the transmission of the disease (OIE, 2013; Tuppurainen et al., 2017). The highest number of outbreaks over the 16 years were documented in the north-west, central, west and south-western parts of the country, which receive relatively high rainfall for a reasonable period. This creates favourable conditions for the replication and survival of blood feeding arthropods assumed responsible for transmission of LSD (Ecotravelworldwide; Woods, 1990; Gari et al., 2010; Ayelet et al., 2014). However, the low disease reporting rate $(<47 \%)$ in Ethiopia should be taken as a limitation in relation to these findings. The reporting rate can be improved through establishing a national disease reporting system based on information technology and capacity building training.

Knowledge of the level of LSD morbidity and mortality is also important for estimating its impact. Morbidities vary across geographical regions (Chapter 3, 4, 6) and they are moderate at individual animal level (10.1\%-26.5\%) but high at herd level (51.0\%-82.3\%). In central and north-western Ethiopia, the true sero-prevalence of LSD at animal level was $26.5 \%$ and at herd level $51.0 \%$ (Chapter 3). A morbidity of $21.2 \%$ at individual animal level was estimated based on a questionnaire survey (Chapter 6). In dominantly crop-livestock system, the animal level morbidity was $10.1 \%$ while in intensive infected herds it was $17.5 \%$ (Chapter 4). These morbidity levels are in agreement with previous research findings (Woods, 1988; Ahmed and Zaher, 2008; Gari et al., 2012; Ayelet et al., 2013; OIE, 2013; Tuppurainen et al., 2017). The regional variation in morbidity might be related to differences in 
geographic location and climate, breed of animals, herd immunity level, management system, and distribution, abundance and efficiency in transmitting LSDV of vector populations (FAO; Davies, 1991; Tuppurainen and Oura, 2012; FAO, 2013; Al-Salihi, 2014). Morbidity of $100 \%$ may occur when LSDV is introduced in a very susceptible cattle population and as low as $2 \%$ in resistant populations (Davies, 1991; Murphy et al., 1999; Tuppurainen et al., 2017). Davies (1991) reported both low and high morbidity in different herds during the same epidemic. The $82.3 \%$ herd morbidity level (Chapter 6 ) is higher than previously reported levels in Ethiopia. This may be related to the way herds were selected for the study being based on the herd owner's capability to describe the main epidemiological and clinical features of LSD. Hence, owners' that had experienced an LSD outbreak in their herd were more likely to be included in the questionnaire survey than owners of unaffected herds were. This selection bias, therefore, might lead to an inflated herd level morbidity.

LSD mortality is generally low: $0.7 \%$ and $4.0 \%$ at animal level in dominantly crop-livestock herds and in intensive infected herds, respectively (Chapter 4 ); $4.5 \%$ and $24.3 \%$ at animal and herd level, respectively (Chapter 6); and $1.4 \%$ in a partly vaccinated cattle population (Chapter 5 ). These outcomes are within the range of previous reports (Woods, 1990; Murphy et al., 1999; Babiuk et al., 2008; Gari et al., 2008; Ayelet et al., 2013; Ayelet et al., 2014). The observed variation in mortality between different study herds might be explained by the health, physiological and nutritional status of the affected animals, susceptibility of the breed and secondary bacterial infections (Davies, 1991; Babiuk et al., 2008). In Ethiopia, previous research reports indicate LSD mortality variation from $0.5 \%$ in the north-western, western and central parts of the country (Mebratu et al., 1984), $1.8 \%$ in southern and $2.8 \%$ northern (Gari et al., 2008) to 5.4\% in central (Ayelet et al., 2014) parts of Ethiopia.

A number of factors affect the occurrence and spread of LSDV (Woods, 1990). Age group, contact with other animals and presence of water bodies were identified as important risk factors in relation to LSD occurrence (Chapter 3 ). Cattle in low laying wet areas suffer from the largest morbidity (Chapter 3), most likely related to more abundant vector populations. Previous research findings indicate that breed is associated with LSD occurrence (Davies, 1991; Gari et al., 2011; OIE, 2013; Abera et al., 2015) and this is in line with the current vaccine efficacy study (Chapter 5). However, the sero-prevalence and risk factor study indicate that breed was not associated with LSD occurrence (Chapter 3); this might be potentially due to the distribution of the breeds among study areas. Most of the crossbred animals were in less affected districts. Adult animals were more frequently affected by LSDV than young animals and calves (Chapter 3). This corroborates what Abera et al. (2015) and Elhaige et al. (2017) who also reported that LSD is more prevalent in adult 
cattle than younger animals. Contrary to what Gari et al. (2010) reported, altitude is not identified as a risk factor in the current study which is in agreement with Abera et al. (2015) who described a non-significant association between agro-climatic zones (midland and highland) and LSDV antibodies. In other studies, factors such as herd size (Hailu et al., 2014), introduction of new cattle to the herd, and use of communal grazing or watering points have been reported as a risk for LSD occurrence in a herd (Gari et al., 2010; Hailu et al., 2014).

\subsubsection{LSD outbreak seasonality and transmission dynamics}

LSD epidemics show a clear seasonal pattern. The number of LSD outbreaks was higher in the months after the long rainy season compared to other seasons (Chapter 2). It was above average for the months September-January and below average for February to August. The highest, and significant, correlation ( $r=0.55)$ between monthly number of LSD outbreaks and monthly rainfall at lag 3 indicates that the rainfall from June to September might be responsible for the increased number of outbreaks from September to January. Other research also shows the seasonality of LSD epidemics following the commencement of rains and the subsequent emergence of large numbers of vectors (Woods, 1988; Davies, 1991; Carn, 1993). The rapid spread of LSD with increased activity of potential vectors was observed in several African countries following the seasonal rains (Davies, 1991). Abundant blood-feeding arthropod populations in association with wet and warm weather conditions were noticed in LSD outbreaks in South Africa and Middle East countries (Weiss, 1968; Tuppurainen and Oura, 2012). Gari et al. (2010) reported a positive and significant association between LSD occurrence and increased bitingflies activity in various agro-climatic zones of Ethiopia and they indicate that the biting fly population starts to build up from April and reaching a maximum in September. In Israel, LSD outbreaks were associated with abundant Stomoxys calcitrans populations (Kahana-Sutin et al., 2016). Heavy seasonal rainfall combined with high temperature is a suitable condition for build-up of a blood-feeding arthropod population that in turn increases the probability of LSDV transmission (Tuppurainen and Oura, 2012).

The average length between two LSD outbreak episodes was 4.5 years (Chapter 2). This is close to the estimated 4.6 years reoccurrence time assuming a major outbreak size of $67 \%$ for an epidemic with a reproduction ratio ( $R$ ) of 1.2 (as estimated in the vaccine efficacy study for unvaccinated animals, Chapter 5 ) and an annual replacement rate of $25 \%$. At the threshold level $(R=1)$, the corresponding reoccurrence time equals 4.6 years (Figure 7.1). The outbreak cycle of 4.5 year is shorter compared to Woods (1988) who reported 5 or 6 year intervals. This might be 
related to censoring of districts not reporting reoccurrence of the disease at the end of 2015 , as their period without LSD outbreaks might go further beyond the study period. The pattern of recurrence of LSD epidemics may vary between regions depending on herd susceptibility, and extent and frequency of vaccination (Woods, 1990). This corroborates the observation that some districts reported outbreaks after one year of quiescence, whereas others reported an outbreak after a relatively long period (13 years) without LSDV (Chapter 2 ).

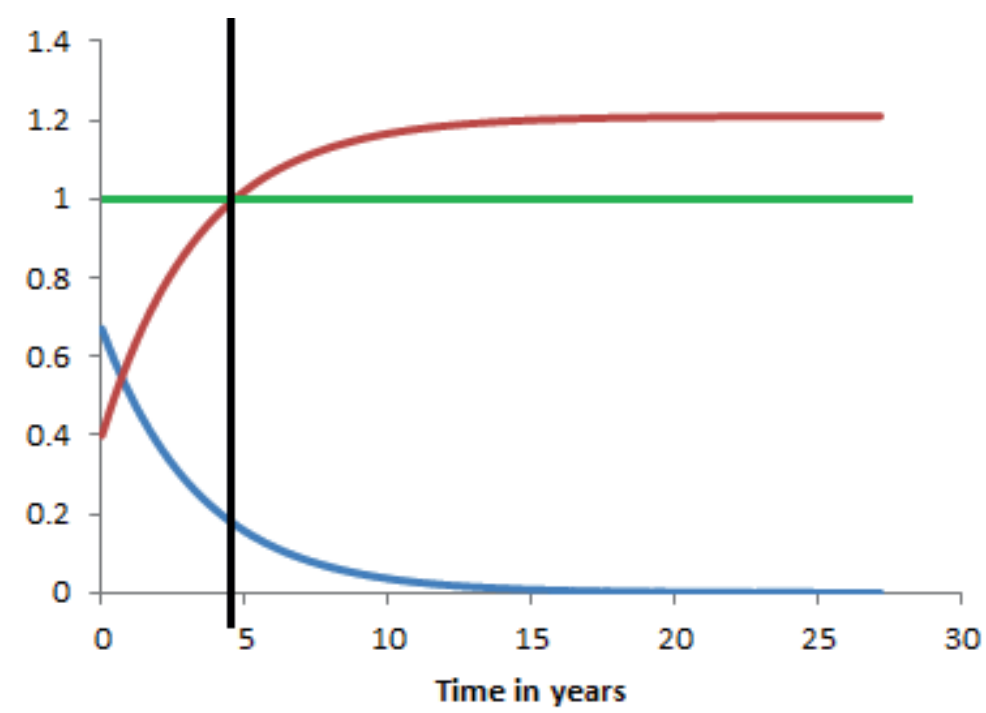

Figure 7.1. Waiting time for the reoccurrence of LSD in a district/area in Ethiopia in relation to reproduction ratio (red line) and the fraction of positives in the population (blue line). The horizontal line (green) indicates the threshold level of $R=1$, and the vertical line (black) shows the average recurrence time.

Knowledge of transmission rate parameters is very important to design effective sets of measures that help to control the disease. For LSD, the transmission rates were low, 0.072 and 0.076 per day between animals in dominantly croplivestock area and intensive farms, respectively (Chapter 4). Furthermore, the reproduction ratios of 1.07 and 1.09 between animals in dominantly crop-livestock area and in intensive farms, respectively, are low. This indicates that an affected animal is responsible for transmission of LSD only to one other animal in most of the cases during its infectious period. This might be the reason why in most of the LSD epidemics usually only 5-45\% of the animals are affected (OIE, 2013). Woods (1988) describe the very slow spread nature of the disease by coining it "a creeping 
pandemic" and that the disease took more than half a century to spread in most African countries (Woods, 1988). The low transmission rate finding might urge to reconsider a claim that transmission of LSDV via direct contact is negligible (Carn and Kitching, 1995; Magori-Cohen et al., 2012). The low R value might explain why susceptible animals that had close contact with infected animals do not get readily infected.

The transmission study showed that transmission rate was highest in the wet season, period 2 (Chapter 4), but the highest number of LSD outbreaks was reported in October (the beginning of the dry season, period 3) (Chapter 2). This discrepancy might be attributed to the reporting period. In Ethiopia, the monthly disease occurrence is reported at the end of each month or at the beginning of the next month, this might be the reason for the highest number of outbreaks at the beginning of the dry season.

\subsubsection{LSD economic impacts}

In general, disease affects the benefits obtained from livestock either by reducing production and productivity, killing animals or by causing consumer demand drops which may lead to collapse of communities depending on them (Perry and Grace, 2009). Chapter 6 presents the economic impact assessment of LSD in both subsistence crop-livestock and commercial farming systems. Financial losses of USD 325 (local Zebu) and USD 1250 (Holstein-Friesian local cross cattle) per dead animal, USD 63 (local Zebu cow) and USD 216 (Holstein-Friesian local cross cow) per lactating cow, USD 36 per affected ox and USD 5 diagnostic and medication cost per affected animal were estimated. Besides, total economic losses of USD 489 in subsistence herds and USD 2735 in commercial farms were estimated at herd level for an LSD outbreak. Recall bias was potentially present at the time of data collection and therefore these estimates can be under- or overestimated. These losses are larger than the loss reported earlier for LSD as well as for other contagious and transboundary disease including FMD in Ethiopia (Gari et al., 2011; Alemayehu et al., 2013; Jemberu et al., 2014). Gari et al. (2011) estimated the financial loss in LSD infected herds at USD 6.43 per head for local Zebu and USD 58 per head for crossbred cattle. Alemayehu et al. (2013) reported a total loss of USD 667,786 in feedlots in and around Adama where 18,864 bulls were at risk during the outbreak. The loss reported in this study is, however, less than what Abutarbush (2014) reported: $£ 486$ (about USD 800) per animal.

The estimated loss due to LSD in both subsistence and commercial farms are huge for producers in a developing country like Ethiopia. In subsistence farms the burden was $40.2 \%$ of the total annual expenditure (USD 1216) of an average 
household for the system (CSA, 2012) which is taken as a proxy of income, whereas in commercial farms the burden was $143.7 \%$ of the total annual expenditure (USD 1903) estimated (CSA, 2012). The loss in both cases is over $10 \%$ of the income which is set as a benchmark for a catastrophic shock (Shankar et al., 2012).

It is obvious that LSD epidemics have huge economic consequences for farmers and other stakeholders (for example traders). Apart from the direct losses due to the morbidity and mortality of animals and the indirect losses related to the costs of disease control and prevention, losses are caused by long-term consequences due to market disruption, production interruption, additional repopulation costs, animal welfare and price effects (Meuwissen et al., 1999; Otte and Chilonda, 2001). The consequential losses related to trade restrictions could be even more important for Ethiopia because livestock sector contributes $12 \%$ of the annual foreign exchange earnings (NBE, 2014). Control measures, therefore, should be put in place to mitigate LSD burdens on the economy.

\subsubsection{LSD control opportunities}

Experiences from other affected countries showed that control of LSD depends on early detection of the index case, immediate stamping-out of all infected and in-contact animals, quarantine, strict movement control, and vaccination (Tuppurainen et al., 2017). Vaccination with a live attenuated vaccine is reported as the effective way to control the spread of LSDV (Tuppurainen et al., 2017). The KS1 O-180 vaccine confers some level of protection to LSD by reducing the susceptibility of vaccinated cattle (Chapter 5 ). Vaccination was also associated with less severe LSD symptoms. However, the estimated reproduction ratio in both vaccinated and unvaccinated cattle indicate that LSD virus does transmit in cattle populations, regardless of their vaccination status, and a major outbreak may occur (Chapter 5). This shows that immunizing animals with KS1 0-180 vaccine will not help much to control and eliminate LSD by bringing the reproduction ratio below the threshold level. To the worst, some people associate the occurrence of the disease in vaccinated animals with vaccination. However, previous research findings clearly indicate that the isolates causing LSD in Ethiopia are different from the vaccine strain (Gelaye et al., 2015) and the current vaccine efficacy study supports this, meaning that the vaccine is not responsible for the occurrence of LSD in vaccinated cattle. Furthermore, the vaccine has been successfully used in Kenya against LSDV without adverse reactions (Davies and Mbugwa, 1985; Tuppurainen et al., 2014).

Despite the KS1 0-180 vaccine is not competent enough to give full protection against LSDV infection, it currently is being used as the main control method in Ethiopia (Ayelet et al., 2013; Gari et al., 2015). The partial budget analysis 
indicated a positive net profit of USD 136 (USD 56 for subsistence farm herds and USD 283 for commercial herds) and a marginal rate of return (MRR) of 15.14 (11.29 in subsistence and 10.10 in commercial herds) per herd by vaccinating the animals against LSD (Chapter 6). This shows that LSD control with vaccination is economically beneficial by reducing losses via decreasing susceptibility of vaccinated animals to the disease and reducing its severity in infected animals. The benefit from vaccination could be more if a vaccine like the Neethling virus vaccine, which is claimed to reduce morbidity (Ben-Gera et al., 2015), is used. The low estimates for transmission rates and reproduction ratios (Chapter 4 ) also indicate the possibility of controlling the disease with moderate level of vaccination coverage by efficacious LSD vaccine. Vaccination complemented with other methods such as movement control and removing severely affected animals is presumed effective to control LSD epidemics (Ben-Gera et al., 2015; EFSA AHAW Panel, 2015).

It is now clear that the occurrence of LSD in areas/districts is cyclic which explains its endemicity in the country (Chapter 2). Once the epidemic is over in a certain area that area may remain free from LSD for some years. A carrier state of LSD recovered animals has not been observed. Therefore, resurgence of an LSD epidemic into a free area occurs either by introduction of infected animals or by blood feeding insects. Thus, new animals should be quarantined before they enter to a free area and banning free and unauthorized animal movement could be taken as an option for the control of LSD in Ethiopia. Stamping out of infected animals and insect control options might not be feasible in resource poor countries. In other parts of the world, insecticides are frequently used to control LSD outbreaks, even though their effectiveness in controlling LSD spread is not yet proven (EFSA AHAW Panel, 2015). Stamping out infected and in contact animals is considered as best option when LSD occurs for the first time in a free country.

Currently, Ethiopia lacks a well-designed control strategy for LSD. Thus, based on the findings presented in Chapter 2 to 6 , it is possible to formulate a control program, which encompasses vaccination, isolation and removing of infected animals, and movement control. Vaccination should be undertaken regularly ahead of the onset of the main rainy season, starting in the most risky geographical areas. Susceptible adult cattle should be vaccinated annually and calves of six months and older, born from immunized dams, should be vaccinated as soon as possible to ensure adequate protection against LSD. Calves over 10 days of age may be vaccinated, if they born from unimmunized cows. However, a more competent vaccine should replace the incompetent KS1 0-180 vaccine, which is currently in use for the control of LSD. Movement of animals from infected to free areas should be banned and live cattle open-air local markets should be closed during LSD outbreaks. 


\subsection{Practical implications}

In Chapter 2, the geographical distribution and seasonal pattern of LSD in Ethiopia has been described using the outbreak reports from 2000-2015. A better understanding of the spatiotemporal distribution of an infectious disease is likely to result in a formulation of better disease control and prevention strategies (Grassly and Fraser, 2006; Alkhamis and VanderWaal, 2016) by specifying where and when particular attention should be paid to control the disease. Furthermore, the forecasted results (Chapter 2) are useful in alerting and helping policy makers to formulate epidemic preparedness plans to prevent and control the occurrence of the disease.

LSD occurrence varies from place to place within the country depending on the risk factors. Areas endowed with surface water bodies (rivers, lakes, ponds, dams, irrigated and swampy areas) and that receive high amount of rainfall are more affected by LSD (Chapter 2 and 3). This suggests that these high-risk areas should get priority in the application of control measures to reduce LSD transmission and economic losses. Risk maps can be drawn based on the findings on potential risk factors that can help in undertaking targeted disease surveillance and control activities (Chapter 2 and 3). Thus, understanding the risk factors and occurrence of LSD may assist in the control and prevention of the diseases in cattle population.

Knowledge of within herd transmission is necessary in disease controlling. The per day transmission rate of the disease between animals was higher at the beginning of the epidemic compared to the later stage of the epidemic (Chapter 4). This result might give a clue that starting implementation of control measures at an early stage of the outbreak or ahead of the outbreak is necessary to halt the spread of the disease. The reproduction ratio, being a parameter that reflects the transmission dynamics of the disease, estimated in dominantly crop-livestock area and intensive farm animals was low in magnitude but above the threshold level (Chapter 4). The reproduction ratios estimated in unvaccinated and vaccinated animals were also above the threshold level but low (Chapter 5). This indicates that the epidemic would spread and affect susceptible cattle, but implementation of appropriate control measures might bring $\mathrm{R}$ to below 1 and the disease under control. If a $100 \%$ effective vaccine is being used, the disease can be brought under control by vaccinating at least $18 \%$ (based on the $\mathrm{R}$ of 1.21 for an unvaccinated population) of the susceptible cattle population (Chapter 5 ). This means that herd immunity can be achieved by vaccinating a moderate number of animals against LSD. This critical vaccination coverage is on the assumption of $100 \%$ vaccine efficacy, but 
in reality the vaccine efficacy is usually less than $100 \%$, so that a vaccination coverage of more than what indicated here might needed to control the disease.

Though livestock contributes a significant portion to the national economy and the fulfilment of the livelihood needs of the poor farmers, the sector often is under-appreciated and inappropriately funded when public resources are being allocated (Perry and Grace, 2009). This discrepancy might be related to lack of understanding of the contribution of livestock. In relation to this, the huge per head and per herd financial loss caused by LSD (Chapter 6 ) might be helpful in alerting and changing the attitude of the policy makers towards the sector and LSD control. The role of livestock should not be seen only as an issue of food security and income, but rather encompasses nutrition security which is closely linked to the wellbeing of the producers and the general population.

The economic impact of LSD, which is greater than many of the endemic transboundary animal diseases including FMD in Ethiopia, urges to design an effective LSD control strategy, which is non-existent to date in the country. Being well aware of the economic impact of LSD, the herd owners are expected to be fully cooperative for the implementation of control measures; particularly for vaccination, if a competent vaccine is available and the vaccination costs continues to be borne by the government.

The study in Chapter 6 also showed that cattle selling is practiced during LSD outbreaks in open-air local markets. This has an implication in the spread of the disease between areas. To halt the progress of the disease epidemics it will be necessary to close the local market and implement some degree of animal movement restriction at least during the outbreak. A strong extension work, however, is needed as subsistence farmers have high resistance against animal movement control and closure of a local market.

Results from previous researches indicate that the KS1 0-180 vaccine has shortcomings in protecting vaccinated animals (Ayelet et al., 2013; Gari et al., 2015; Gelaye et al., 2015) which was confirmed by results described in Chapter 5 . The reproduction ratio estimated in unvaccinated and vaccinated cattle populations also gave additional insight in the effect of the vaccine on the disease dynamics at population level and emphasises the necessity of a more competent vaccine to control the disease.

\subsection{Future research recommendations}

The geographic distribution of vector-borne diseases tends to be influenced by climate whereas disease transmission dynamics, the timing and intensity of 
outbreaks by variations in weather such as temperature, rainfall, and humidity (Institute of Medicine, 2008; Tuppurainen et al., 2017). However, due to information constraints, the influence of temperature and humidity on the LSD outbreak occurrences could not be assessed in the spatiotemporal study (Chapter 2). Hence, future study in the area should address this.

Circumstantial evidence observed in the previous and the current studies indicate that the occurrence of LSD is associated with increased abundance of arthropod vectors. However, little information is available on the specific vectors responsible for the transmission of LSD. Field observations suggest that insects like mosquitoes, Culicoides, Tabanids, and Glossina spp. may transmit the virus mechanically (Davies, 1991). Weiss (1968) reported the isolation of LSD virus from Stomoxys calcitrans and Biomyia fasciata caught on infected cattle and Chihota et al. (2001) demonstrated female Aedes aegypti mosquitoes to transmit LSD virus for 26 days post-feeding on experimentally infected animals. Tuppurainen et al. (2011; 2013a,b) and Lubinga et al. (2015) have reported a possible role of hard ticks in the transmission of LSDV. Vectors responsible for transmission may vary between affected regions depending on the geographic location and climate of the region (Davies, 1982; Tuppurainen et al., 2017). So, research on the candidate vectors responsible for the transmission of LSD in a region is needed to enhance the control efforts. Furthermore, the use of insecticides and insect repellents as a means for the LSD control should be assessed for their applicability, effectiveness and safety.

The reproduction ratio is a key parameter determining the level of disease transmission and disease persistence in a population (Velthuis et al., 2003). It is of value in assessing in real time whether interventions have effect in controlling the disease. The reproduction ratio is a composite measure of infectiousness and susceptibility, but to date the infectious period of cattle infected with LSDV and the role of the virus in the environment towards the transmission is not known. Further research into these, preferentially under experimental conditions, will lead to a better understanding of LSD transmission dynamics and estimates of LSD transmission parameters, and thus to a better control strategy.

Assessing the impact of diseases is necessary but not sufficient to proceed directly to their control. It is not automatic that a disease with high economic impact should be tackled, different factors such as availability of effective control methods, technical feasibility, economical benefit, political willingness and the like, determine the move to action (Perry and Grace, 2009). Control measures such as vaccination, movement control, stamping out, and vector control can be employed separately or in combination to control LSD (Tuppurainen et al., 2017), but before applying them, their effectiveness, technical feasibility and cost effectiveness in controlling the 
disease should analysed. Transmission experiments could be useful in estimating the effect of (combinations of) interventions measures. Besides, development of a new effective and safe vaccine should get priority in future research undertakings.

\subsection{Main conclusions of the thesis}

With the research described in the thesis, insight in LSD epidemiology, financial consequence and effect of vaccination as a control means has increased. The insight is crucial in order to better prevent and control the disease in the future. Based on the findings of this thesis, the following conclusions can be drawn:

- Lumpy skin disease is endemic in Ethiopia. Districts are hit by an outbreak on average each 4.5 years (Chapter 2 ).

- LSD outbreak incidence varies over space and time and shows an increasing trend over time (Chapter 2). Outbreaks are high at the end of the long rainy season (from September to January) and more numerous in areas receiving relatively high rainfall (north-west, central, west and south-western parts of the country) for a reasonable period of time (Chapter 2).

- $\quad$ LSD sero-prevalence varies across districts and it is moderate at individual animal level (26.5\%) but high at herd level (51.0\%) (Chapter 3 ).

- Animals at adult age and that live in an area endowed with abundant surface water bodies are at relatively higher risk for LSD infection (Chapter 3). Cattle populations exposed to these risks should get priority in LSD control programs.

- The 0.325 per day survival rate of the infectious virus in the environment increases the infectivity of LSD affected animals by $50 \%$. The daily LSDV transmission rate between animals is higher at the beginning of the outbreak compared to the later stage of the outbreak. Thus, implementation of control measures at early stage of the outbreak is helpful to halt the spread of the disease (Chapter 4).

- The estimated reproduction ratio of LSD is about 1.1 and thus above the threshold level but low in magnitude which implies that every affected animals transmit the disease only almost to one other animal (Chapter 4).

- $\quad$ KS1 0-180 vaccine has poor efficacy in protecting cattle populations with partial vaccination coverage against clinical LSD, but it significantly reduces severity of the disease (Chapter 5 ).

- LSD's impact in terms of production losses and control costs is high. The losses are mainly from morbidity and mortality of cattle and are the greatest 
in highly productive animals. Commercial farmers are more affected economically than subsistence crop related farmers (Chapter 6).

- Vaccination is economically beneficial as it reduces losses by decreasing susceptibility to LSDV and severity in vaccinated infected animals (Chapter 6) despite a poor efficacy of KS1 O-180 vaccine in protecting cattle against clinical LSD.

\section{References}

Abera, Z., Degefu, H., Gari, G., Kidane, M., 2015. Sero-prevalence of lumpy skin disease in selected districts of West Wollega zone, Ethiopia. BMC Vet. Res. 11, 135.

Abutarbush, S.M., 2014. Efficacy of vaccination against lumpy skin disease in Jordanian cattle. Vet. Rec. 175, 302.

Ahmed, W.M., Zaher, K.S., 2008. Observations on lumpy skin disease in local Egyptian cows with emphasis on its impact on ovarian function. Afr. J. Microbiol. Res. 2, 252-257.

Al-Salihi, K.A., 2014. Lumpy skin disease: Review of literature. MRSVA 3, 6-23.

Alemayehu, G., Zewde, G., Admassu, B., 2013. Risk assessments of lumpy skin diseases in Borena bull market chain and its implication for livelihoods and international trade. Trop. Anim. Health Prod. 45, 1153-1159.

Alkhamis, M.A., VanderWaal, K., 2016. Spatial and temporal epidemiology of lumpy skin disease in the Middle East, 2012-2015. Front. Vet. Sci. 3, 19.

APHRD, 2012. Biannual epidemiology newsletter, Animal and Plant Health Regulatory Directorate (APHRD), Ministry of Agriculture, Addis Ababa, Ethiopia 2, 1-21.

AU-IBAR, 2014. Pan African animal resources yearbook. African Union InterAfrican Bureau for Animal Resources (AU-IBAR) Nairobi, Kenya.

Ayelet, G., Abate, Y., Sisay, T., Nigussie, H., Gelaye, E., Jemberie, S., Asmare, K., 2013. Lumpy skin disease: preliminary vaccine efficacy assessment and overview on outbreak impact in dairy cattle at Debre Zeit, central Ethiopia. Antivir. Res. 98, 261-265.

Ayelet, G., Haftu , R., Jemberie, S., Belay, A., Gelaye, E., Sibhat, B., Skjerve, E., Asmare, K., 2014. Lumpy skin disease in cattle in central Ethiopia: outbreak investigation and isolation and molecular detection of lumpy skin disease virus. Rev. Sci. Tech. Off. Int. Epiz. 33, 877-887. 
Babiuk, S., Bowden, T.R., Boyle, D.B., Wallace, D.B., Kitching, R.P., 2008. Capripoxviruses: an emerging worldwide threat to sheep, goats and cattle. Transbound. Emerg. Dis. 55, 263-272.

Ben-Gera, J., Klement, E., Khinich, E., Stram, Y., Shpigel, N.Y., 2015. Comparison of the efficacy of Neethling lumpy skin disease virus and x10RM65 sheep-pox live attenuated vaccines for the prevention of lumpy skin disease - The results of a randomized controlled field study. Vaccine 33, 4837-4842.

Carn, 1993. Control of capripoxvirus infections. Vaccine 11, 1275-1279.

Carn, V.M., Kitching, R.P., 1995. An investigation of possible routes of transmission of lumpy skin disease virus (Neethling). Epidemiol. Infect. 114, 219-226.

Chihota, C.M., Rennie, L.F., Kitching, R.P., Mellor, P.S., 2001. Mechanical transmission of lumpy skin disease virus by Aedes aegypti (Diptera: Culicidae). Epidemiol. Infect. 126, 317-321.

CSA, C.S.A., 2012. Household Consumption and Expenditure (HCE) Survey 2010/11, Analytical Report. Statistical Bulletin 563. Central Statistical Agency (CSA), Federal Democratic Republic of Ethiopia, Addis Ababa.

Davies, F.G., 1982. Observations on the epidemiology of lumpy skin disease in Kenya. J. Hyg. Camb. 88, 95-102.

Davies, F.G., 1991. Lumpy skin disease, an African capripox virus disease of cattle. Br. Vet. J. 147, 489-503.

Davies, F.G., Mbugwa, G., 1985. The alterations in pathogenicity and immunogenicity of a Kenya sheep and goat pox virus on serial passage in bovine foetal muscle cell cultures. J. Comp. Pathol. 95, 565-572.

Ecotravelworldwide, Ethiopia weather and climate zones. http://www.nationalparks-worldwide.info/eaf/ethiopia/ethiopiaweather.html. Accessed 18 May 2016.

EFSA AHAW Panel, E.P.o.A.H.a.W., 2015. Scientific opinion on lumpy skin disease. EFSA J. 2015, 13

Elhaig, M.M., Selim, A., Mahmoud, M., 2017. Lumpy skin disease in cattle: Frequency of occurrence in a dairy farm and a preliminary assessment of its possible impact on Egyptian buffaloes. Onderstepoort J. Vet. Res. 84, 1. https://doi.org/10.4102/ojvr. v84i1.1393.

FAO, Lumpy skin disease of cattle: a growing problem in Africa and the near East. http://www.fao.org/docrep/u4900t/u4900t0d.htm. Accessed 26 April 2017.

FAO, 2013. Emergence of lumpy skin disease in the Eastern Mediterranean Basin countries. EMPRES WATCH, Rome, 1-6. 
Gari, G., Abie, G., Gizaw, D., Wubete, A., Kidane, M., Asgedom, H., Bayissa, B., Ayelet, G., Oura, C.A., Roger, F., Tuppurainen, E.S., 2015. Evaluation of the safety, immunogenicity and efficacy of three capripoxvirus vaccine strains against lumpy skin disease virus. Vaccine 33, 3256-3261.

Gari, G., Biteau-Coroller, F., LeGoff, C., Caufour, P., Roger, F., 2008. Evaluation of indirect fluorescent antibody test (IFAT) for the diagnosis and screening of lumpy skin disease using Bayesian method. Vet. Microbiol. 129, 269-280.

Gari, G., Bonnet, P., Roger, F., Waret-Szkuta, A., 2011. Epidemiological aspects and financial impact of lumpy skin disease in Ethiopia. Prev. Vet. Med. 102, 274283.

Gari, G., Grosbois, V., Waret-Szkuta, A., Babiuk, S., Jacquiet, P., Roger, F., 2012. Lumpy skin disease in Ethiopia: seroprevalence study across different agroclimate zones. Acta Trop. 123, 101-106.

Gari, G., Waret-Szkuta, A., Grosbois, V., Jacquiet, P., Roger, F., 2010. Risk factors associated with observed clinical lumpy skin disease in Ethiopia. Epidemiol. Infect. 138, 1657-1666.

GebreMariam, S., Amare, S., Baker, D., Solomon, A., 2010. Diagnostic study of live cattle and beef production and marketing: Constraints and opportunities for enhancing the system. http://bdsknowledge.org/dyn/bds/docs/800/Ethiopia-livestock-valuechain-diagnostic-july-201.pdf. Accessessed 15 February 2017.

Gelaye, E., Belay, A., Ayelet, G., Jenberie, S., Yami, M., Loitsch, A., Tuppurainen, E., Grabherr, R., Diallo, A., Lamien, C.E., 2015. Capripox disease in Ethiopia: Genetic differences between field isolates and vaccine strain, and implications for vaccination failure. Antivir. Res. 119, 28-35.

Grassly, N.C., Fraser, C., 2006. Seasonal infectious disease epidemiology. Proc. R. Soc. B. 273, 2541-2550.

Hailu, B., Tolosa, T., Gari, G., Teklue, T., Beyene, B., 2014. Estimated prevalence and risk factors associated with clinical Lumpy skin disease in north-eastern Ethiopia. Prev. Vet. Med. 115, 64-68.

Institute of Medicine, 2008. Vector-borne diseases: understanding the environmental, human health, and ecological connections. The National Academies Press Washington, DC. pp. 350.

Jemberu, W.T., Mourits, M.C., Woldehanna, T., Hogeveen, H., 2014. Economic impact of foot and mouth disease outbreaks on smallholder farmers in Ethiopia. Prev. Vet. Med. 116, 26-36.

Kahana-Sutin, E., Klement, E., Lensky, I., Gottlieb, Y., 2016. High relative abundance of the stable fly Stomoxys calcitrans is associated with lumpy skin disease 
outbreaks in Israeli dairy farms. Med. Vet. Entomol., doi: 10.1111/mve.12217.

Lubinga, J.C., Tuppurainen, E.S., Mahlare, R., Coetzer, J.A., Stoltsz, W.H., Venter, E.H., 2015. Evidence of transstadial and mechanical transmission of lumpy skin disease virus by Amblyomma hebraeum ticks. Transbound. Emerg. Dis. 62, 174-182.

Magori-Cohen, R., Louzoun, Y., Herziger, Y., Oron, E., Arazi, A., Tuppurainen, E., Shpigel, N.Y., Klement, E., 2012. Mathematical modelling and evaluation of the different routes of transmission of lumpy skin disease virus. Vet. Res. 43.

Mebratu, G.Y., Kassa, B., Fikre, Y., Berhanu, B., 1984. Observation on the outbreak of lumpy skin disease in Ethiopia. Rev. Elev. Méd. Vét. Pays Trop. 37, 395-399.

Meuwissen, M.P.M., Horst, S.H., Huirne, R.B.M., Dijkhuizen, A.A., 1999. A model to estimate the financial consequences of classical swine fever outbreaks: principles and outcomes. Prev. Vet. Med. 42, 249-270.

Murphy, F.A., Gibbs, E.P.J., Horzinek, M.C., Studdert, M.J., 1999. Veterinary Virology Academic Press San Diego. pp. 278-291.

NBE, 2014. National Bank Annual Report of 2013-2014. National Bank of Ethiopia (NBE), Addis Ababa Ethiopia.

OIE, 2013. Lumpy skin disease. OIE Technical Disease Cards. OIE.

OIE, 2014. Guidelines for animal disease control. http://www.oie.int/fileadmin/Home/eng/Our_scientific_expertise/docs/p df/A_Guidelines_for_Animal_Disease_Control_final.pdf. Accessed 24 August 2017. .

Otte, M.J., Chilonda, P., 2001. Animal health economics: An introduction. Livestock information, sector analysis and policy branch, animal production and health division. FAO Rome, Italy.

Perry, B., Grace, D., 2009. Review: The impacts of livestock diseases and their control on growth and development processes that are pro-poor. Phil. Trans. R. Soc. B. 364, 2643-2655.

Shankar, B., Morzaria, S., Fiorucci, A., Hak, M., 2012. Animal disease and livestockkeeper livelihoods in Southern Cambodia. Int. Dev. Plann. Rev. 34, 39-63.

Tuppurainen, E.S., Lubinga, J.C., Stoltsz, W.H., Troskie, M., Carpenter, S.T., Coetzer, J.A., Venter, E.H., Oura, C.A., 2013a. Evidence of vertical transmission of lumpy skin disease virus in Rhipicephalus decoloratus ticks. Ticks Tick-borne Dis. 4, 329-333.

Tuppurainen, E.S., Lubinga, J.C., Stoltsz, W.H., Troskie, M., Carpenter, S.T., Coetzer, J.A., Venter, E.H., Oura, C.A., 2013b. Mechanical transmission of lumpy skin 
disease virus by Rhipicephalus appendiculatus male ticks. Epidemiol. Infect. 141, 425-430.

Tuppurainen, E.S., Oura, C.A., 2012. Review: lumpy skin disease: an emerging threat to Europe, the Middle East and Asia. Transbound. Emerg. Dis. 59, 40-48.

Tuppurainen, E.S., Pearson, C.R., Bachanek-Bankowska, K., Knowles, N.J., Amareen, S., Frost, L., Henstock, M.R., Lamien, C.E., Diallo, A., Mertens, P.P., 2014. Characterization of sheep pox virus vaccine for cattle against lumpy skin disease virus. Antivir. Res. 109, 1-6.

Tuppurainen, E.S., Stoltsz, W.H., Troskie, M., Wallace, D.B., Oura, C.A., Mellor, P.S., Coetzer, J.A., Venter, E.H., 2011. A potential role for ixodid (hard) tick vectors in the transmission of lumpy skin disease virus in cattle. Transbound. Emerg. Dis. 58, 93-104.

Tuppurainen, E.S., Venter, E.H., Shisler, J.L., Gari, G., Mekonnen, G.A., Juleff, N., Lyons, N.A., De Clercq, K., Upton, C., Bowden, T.R., Babiuk, S., Babiuk, L.A., 2017. Review: capripoxvirus diseases: current status and opportunities for control. Transbound. Emerg. Dis. 64, 729-745.

Velthuis, A.G.J., De Jong, M.C.M., Kamp, E.M., Stockhofe, N., Verheijden, J.H.M., 2003. Design and analysis of an Actinobacillus pleuropneumoniae transmission experiment. Prev. Vet. Med. 60, 53-68.

Weiss, K.E., 1968. Lumpy skin disease virus. Virol. Monogr. 3, 111-131.

Woods, J.A., 1988. Lumpy skin disease- A review. Trop. Anim. Health Prod. 20, 11-17.

Woods, J.A., 1990. Lumpy skin disease virus. In: Dinter, Z., Morein, B. eds. Virus infections of ruminants. Elsevier Science publishers B. V. Amesterdam. pp. 53-67. 


\section{Summary}





\section{Summary}

Lumpy skin disease (LSD) is an economically important viral disease of cattle caused by lumpy skin disease (Neethling) virus of the genus Capripoxvirus. Morbidity of the disease varies from as low as $2 \%$ to $100 \%$ while mortality is generally considered low (usually less than $5 \%$ ). The disease is endemic in Africa and Middle East countries. In recent times, the disease has also spread into Europe and West Asia.

LSD is an endemic transboundary animal disease that has a significant economic impact on livestock sector. In Ethiopia, the disease causes major economic loss since its introduction in 1981. This loss is associated with low production and productivity, high control and prevention costs and international trade restrictions on cattle and their products. Although the disease is endemic and presumed to have huge economic consequences, little was known about its spatiotemporal distribution, transmission dynamics, predisposing factors, financial loss and efficiency of prevention and control strategies practiced in Ethiopia. The main aim of this study was, therefore, to generate information on LSD epidemiology, its financial consequences and the effect of vaccination as a control means. This information then can be used to formulate a LSD control strategy policy, which currently is lacking in Ethiopia.

The spatiotemporal distribution of LSD in Ethiopia (Chapter 2) was assessed using data extracted from LSD outbreak reports over 16 years (2000-2015) with the objectives to determine the spatial and temporal distribution, and to forecast the future pattern of LSD outbreaks. For this study, LSD outbreak data were obtained from the Federal Veterinary Services Directorate of Ethiopia for the period 20002015 and the monthly mean precipitations for the period 1999-2013 from the Global weather data for SWAT website. The national LSD outbreak incidence was established at district level and its geographical distribution was mapped by administrative zones. Time series analysis was used for depicting temporal patterns of $\operatorname{LSD}$ outbreaks. An ARIMA $(1,1,1) \times(1,1,1) 12$ model was used to forecast the number of LSD outbreaks that are expected to occur between January 2016 to December 2018 (Chapter 2). A total of 3811 LSD outbreaks were reported in Ethiopia between 2000 and 2015, an outbreak being defined as the occurrence of one or more LSD case in a specified geographical area (usually Kebele). LSD was reported at least once over the period in all regional states and city administrations. The national average incidence of district level LSD outbreaks was 5.58 per 16 district years. The incidence varied over space, being the lowest in hot dry lowlands and highest in warm moist highland areas. The occurrence of LSD outbreaks appeared to be 
seasonal, it peaks in October and become lowest in May. In general, the number of LSD outbreaks was above average for the months September-January and below average for February to August. The trend of LSD outbreaks increased over the period 2000-2015. Based on the forecast for the period 2016-2018 the highest number of LSD outbreaks (about 36 outbreaks) will occur in October for all the three years and the lowest in April for the year 2016 and in May for 2017 and 2018 (Chapter 2).

Chapter 3 describes a cross-sectional study that was undertaken in central and north-western parts of Ethiopia to estimate the sero-prevalence and identify the associated risk factors of LSD. Multistage cluster sampling was chosen because the population studied had a nested structure and lists of individual animals were lacking in the study area. Nesting was due to sampling several herds/animals in a kebele (the smallest administrative division in Ethiopia). A total of 2386 cattle serum samples were collected in 30 kebeles and tested for antibodies against LSDV using a virus neutralization test. Data on putative environmental and animal risk factors were collected by interviewing the farmers at the time of blood sample collection. Multivariable mixed effect logistic regression with kebele as random effect was applied to assess the association between LSD sero-positivity and potential risk factors. LSDV antibodies were detected in all study kebeles. The overall animal level and herd level apparent sero-prevalences recorded were $25.4 \%$ and $48.9 \%$, respectively. The true animal level and herd level prevalences were estimated as $26.5 \%$ and $51.0 \%$, respectively. Adult age, contact with other animals and presence of water bodies in the area were identified as the most important risk factors in relation to LSD infection. The intracluster correlation coefficient was 0.104 , the design effect 9.2 and the standard error of the apparent prevalence (animal level) was $2.7 \%$ (Chapter 3 ).

The transmission study described in Chapter 4 was carried out in central and north-western parts of Ethiopia with the aims to understand LSD outbreak dynamics, to quantify the transmission between animals, and to estimate the disease reproduction ratio in dominantly crop-livestock mixed and commercial herd types. Animals in the crop-livestock area (Mota area) were considered as one large herd due to continuous mixing of animals, whereas commercial herds were separate herd with no or little contact with animals of other herds. The infection chain in the herd was monitored by recording the number of susceptible, infected, recovered and newly infected animals (with the date of onset of the infection) at regular visits. The infected animals were assumed to stay infectious for ten days. The transmission of LSD virus between animals was estimated from the relationship between the number of animals newly infected at the end of each observation time interval and 
the number of infectious animals during that same time interval using a SIR epidemic model including a factor for environmental transmission. A generalized linear model was employed for the estimation of transmission parameters. A survival rate of 0.325 per day in the environment for LSDV was used to estimate the transmission parameters. The estimated transmission rate between animals was 0.072 per day in dominantly crop-livestock production system and 0.076 per day in commercial herds. In dominantly crop-livestock system, transmission rates were higher at the beginning of the outbreaks. The reproduction ratios (R) estimated for dominantly crop-livestock production system and commercial production system were 1.07 and 1.09, respectively. Though these R's are greater than 1 , their magnitude is low and implementation of appropriate control measures might reduce $\mathrm{R}$ to below 1 .

The vaccine impact study described in Chapter 5 was carried out in central and north-western parts of Ethiopia with the objectives to assess the efficacy of KS1 0-180 vaccine against natural LSD occurrence and its impact on the transmission and severity of the disease. The study was based on a questionnaire survey and an observational field study. The latter included a total of 2053 cattle (1304 vaccinated and 749 unvaccinated) in 339 infected herds located in 10 sub-kebeles; the questionnaire survey was administered to 224 herd owners. A live attenuated KS1 O-180 vaccine produced by National Veterinary Institute (NVI) of Ethiopia was used for vaccination. Multivariable ordered logistic regression and generalized linear regression were applied to estimate the effect of vaccination on the severity level and on transmission of LSD at sub-kebele level, respectively. Over $60 \%$ of the herd owners reported that the vaccine has a low to very low effect in protecting animals against clinical LSD; almost all of them indicated that the vaccine did not induce any adverse reactions after vaccination. Severity of the disease was significantly reduced in vaccinated compared to unvaccinated animals. Vaccinated animals were more likely to develop less severe disease than unvaccinated animals $(\mathrm{OR}=0.68,95 \% \mathrm{Cl}$ : $0.49-0.96)$. LSD vaccine efficacy for susceptibility was estimated as 0.46 (i.e. the susceptibility effect equals $1-0.46=0.54$ ) while the effect of the vaccine on infectivity was estimated as 1.83 . This indicates that vaccinated animals are half as susceptible to get the disease but once infected the infectivity is almost doubled compared to unvaccinated animals. LSD transmission occurred in both vaccinated and unvaccinated animals, the estimated reproduction ratios were very similar: 1.21 in unvaccinated animals and 1.19 in vaccinated ones. Generally, KS1 0-180 vaccine has poor efficacy in protecting cattle populations with partial vaccination status against clinical LSD, neither by direct clinical protection nor by reducing transmission, and this signifies the urgent need either to improve the quality of the vaccine or to develop potent alternative vaccines that will confer good protection. 
Chapter 6 describes the results of a questionnaire survey targeting to determine the direct financial losses of LSD related to milk loss, draft power loss, mortality and indirect losses due to treatment and vaccination as a means of LSD control in central and north-western parts of Ethiopia. The production systems in the study area were subsistence crop-livestock and commercial dairy production. A total of 243 herd owners from 15 districts ( 34 kebeles and 5 farms) were enrolled in the study. The economic impact of LSD was determined by an estimation of the direct (visible) production losses such as milk loss, mortality loss, and draft power loss, and indirect impacts like control costs. Impacts of the other direct losses such as reduced bodyweight, abortion, infertility, culling, and poorer hide quality were not considered in the study due to lack of information. The losses were estimated per head and per herd. The cost effectiveness of LSD control through vaccination was evaluated using partial budgeting analysis technique. From a total of 4430 cattle (within 243 herds) surveyed, 941 animals (in 200 herds) were reported to be infected. The overall morbidity and mortality at animal level were $21.2 \%$ and $4.5 \%$, and at herd level, these were $82.3 \%$ and $24.3 \%$. LSD is a top ranked disease for cattle producers in Ethiopia. Almost all of the herd owners knew its effect on production and productivity of animals and marketing. They reported that the disease induces weight loss, reduced milk production, draft power loss, death of animals, market instability, infertility, abortion, culling, and hides quality losses (Chapter 6). An overall median loss of USD 375 (USD 325 in local Zebu and USD 1250 in HolsteinFriesian local cross cattle) was estimated per dead animal. The median losses per affected lactating cow were USD 141 (USD 63 in local Zebu cow and USD 216 in Holstein-Friesian local cross cow) and, USD 36 per affected ox. Diagnosis and medication cost per affected animal were estimated at USD 5. The median total economic losses of LSD outbreaks at herd level were USD 1176. The median total herd level economic losses in the subsistence and commercial farm types were USD 489 and 2735, respectively. A positive net profit of USD 136 (USD 56 for subsistence farm herds and USD 283 for commercial herds) per herd was observed in return to LSD vaccine investment.

In Chapter 7, the outcomes of all chapters are linked and discussed. First, the importance of LSD in livestock industry of Ethiopia was emphasised. Next, the findings of the research were organized and discussed. The practical implications, future research outlook and main conclusions of the thesis were also highlighted in Chapter 7. 
About the author 



\section{Curriculum Vitae}

Wassie Molla Abebe was born on the $7^{\text {th }}$ of January 1973 in Estie, Ethiopia. He studied Veterinary Medicine and received a degree of Doctor of Veterinary Medicine from Faculty of Veterinary Medicine, Addis Ababa University, Ethiopia. After graduation he served as district and zonal veterinary officer and research officer under the Bureau of Agriculture of Amhara National Regional State, Ethiopia. Later he joined again Addis Ababa University to pursue his master's degree in Tropical Veterinary Medicine at Faculty of Veterinary Medicine, Addis Ababa University during which he studied the occurrence of salmonellosis in apparently healthy slaughtered sheep and goats. In 2004, he joined Faculty of Veterinary Medicine, University of Gondar as academic staff. In this University he served as department head, research and publication officer and general director of the research and community services of the University. He has been offering various courses, and involved in different research undertakings and actively participated in a range of the university affairs. In September 2013, he joined Wageningen University \& Research to pursue his PhD in Veterinary Epidemiology at Quantitative Veterinary Epidemiology group in Wageningen Institute of Animal Science. His Work was on Bovine lumpy skin disease epidemiology, economic impact and control opportunities in Ethiopia. The results of this thesis were presented in international conferences and published in journals. After completion of his PhD, he will re-join the University of Gondar and can be contacted at: Faculty of Veterinary Medicine, University of Gondar, Gondar, Ethiopia. P. O. Box 196. Email: mollawassie@yahoo.com. 


\section{Publications}

Molla, W., de Jong, M. C. M., Frankena, K., 2017. Temporal and spatial distribution of lumpy skin disease outbreaks in Ethiopia in the period 2000 to 2015 . BMC Vet. Res. 13, 310. DOI 10.1186/s12917-017-1247-5.

Molla, W., Frankena K., de Jong, M. C. M., 2017.Transmission dynamics of lumpy skin disease in Ethiopia. Epidemiol. Infect. 145, 2856-2863. doi: $10.1017 /$ S0950268817001637.

Molla, W., Frankena, K., Gari, G., de Jong, M.C.M., 2017. Field study on the use of vaccination to control the occurrence of lumpy skin disease in Ethiopian

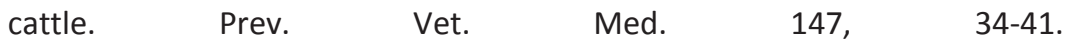
http://dx.doi.org/10.1016/i.prevetmed.2017.08.019.

Molla, W., de Jong, M.C.M., Gari, G., Frankena, K., 2017. Economic impact of lumpy skin disease and cost effectiveness of vaccination for the control of outbreaks in Ethiopia. Prev. Vet. Med. 147, 100-107. http://dx.doi.org/10.1016/i.prevetmed.2017.09.003.

Hailu, D., Gelaw, A., Molla, W., Garedew, L., Cole, L., Johnson, R., 2015. Prevalence and antibiotic resistance patterns of Salmonella isolates from lactating cows and in-contact humans in dairy farms, Northwest Ethiopia. J. Environ. Occup. Sci. 4, 171-178.

Molla, W., Teshome, D,. Almaw, G., Temesgen, W., Alemu, S., 2015. Poultry coccidial infection in local chickens from three selected districts of North Gondar zone, Ethiopia. Ethiop. Vet. J. 19, 1-9.

Melaku, A., Bogale, B., Chanie, M., Molla, W., Almaw, A., 2014. A three year followup study on the occurrence of bovine ehrlichiosis (cowdriosis) at Gondar University dairy farm. J. Vet. Med. Anim. Health, 6, 83-90.

Yohannis, M. and Molla, W., 2013. Prevalence, risk factors and major bacterial causes of bovine mastitis in and around Wolaita Sodo, Southern Ethiopia. Afr. J. Microbiol. Res. 7, 5400-5405.

Kasaye, S., Molla, W., Amini, G. 2013. Prevalence of camel tuberculosis at Akaki abattoir in Addis Ababa, Ethiopia. Afr. J. Microbiol. Res. 7, 2184-2189.

Jemberu, W. T., Molla, W., Almaw, G., Alemu, S. 2013. Incidence of rabies in humans and domestic animals and people's awareness in North Gondar Zone, Ethiopia. PLoS Negl. Trop. Dis. 7, e2216.

Asmare, K., Sibhat, B., Molla, W., Ayelet, G., Shiferaw, J., Martin, A. D., Skjerve, E., Godfroidg, J., 2013. The status of bovine brucellosis in Ethiopia with special emphasis on exotic and cross bred cattle in dairy and breeding farms. Acta Trop. 126, 186- 192. 
Belay, E., Molla, W., Amare, A., 2012. Prevalence and economic losses of Bovine fasciolosis in Dessie Municipal Abattoir, South Wollo Zone, Ethiopia. Europ. J. Biol. Sci. 4, 53-59.

Warsame, I., Alemu, S., Temesgen, W. and Molla, W., 2012. Seroprevalence and associated risk factors of camel (Camelus dromedaries) brucellosis in and around Dire Dawa, Ethiopia. Global Veterinaria, 8, 480-2012.

Kassaye, D. and Molla, W., 2012. Seroprevalence of contagious bovine pleuropneumonia at export quarantine centers in and around Adama, Ethiopia. Trop. Anim. Health Prod. 45, 275-279.

Almaw, G., Molla, W. , Melaku, A., 2012. Incidence rate of clinical bovine mastitis in selected smallholder dairy farms in Gondar town, Ethiopia. Ethiop. Vet. J. 16, 93-99.

Molla, W., Haile, H., Almaw, G. and Temesgen, W., 2012. Gastrointestinal helminths of local backyard chickens in North Gondar Administrative Zone, Ethiopia, Revue Méd. Vét., 163, 353-358.

Kassa, S. A., Molla, W., 2012. Seroprevalence of infectious bursal disease in backyard chickens of North West Ethiopia. Scientific Journal of Crop Science, 1, 2025.

Almaw, G., Molla, W. and Melaku, A., 2009. Prevalence of bovine sub clinical mastitis in Gondar town and surrounding area. Livestock Research for Rural Development, 21.

Molla, W., Molla, B., Alemayehu, D., Muckle, A., Cole L., Wilkie, E., 2006. Occurrence and antimicrobial resistance of Salmonella serovars in apparently healthy slaughtered sheep and goats of central Ethiopia. Trop. Anim. Health Prod. $38,455-462$. 


\section{Conference proceedings, abstracts and presentation}

Molla, W., Frankena, K., de Jong, M.C.M., 2017. Transmission dynamics of lumpy skin disease in Ethiopia. Modeling in Animal Health (ModAH) Conference, Nantes, France.

Molla, W., Frankena, K., Gari, G., Kidane, M., Shegu, D., de Jong, M.C.M., 2017. Seroprevalence and risk factors of lumpy skin disease in Ethiopia. The Society for Veterinary Epidemiology and Preventive Medicine Annual Conference Inverness, Scotland.

Molla, W., Frankena, K., Gari, G., de Jong, M. C.M., 2017. Field study on Kenyan sheep and goat pox virus vaccine efficacy in controlling lumpy skin disease outbreak and its impact on reducing disease severity. WIAS Science Day, Wageningen, The Netherlands.

Molla, W., Frankena, K., de Jong, M.C.M., 2016. Temporal and spatial distribution of lumpy skin disease (LSD) outbreaks in Ethiopia (2000-2015). 28th VEEC Symposium, Lelystad, The Netherlands.

Molla, W., Frankena, K., Gari, G., de Jong, M. C.M., 2016. Economic impact of LSD and cost effectiveness of vaccination for the control of outbreak in Ethiopia. First Joint AITVM-STVM Conference, Berlin, Germany.

Molla, W., Frankena, K., Gari, G., de Jong, M. C.M., 2014. Lumpy skin disease occurrence, transmission, cost and impact of vaccination in Ethiopia. WIAS Science Day, Wageningen, The Netherlands. 
Education and training 



\section{Training and supervision plan accomplishment}

The Basic Package (3.0 ECTS)

Year

WIAS Introduction Course, Wageningen, The Netherlands

2013

WIAS Ethics and Philosophy in Life Sciences, Dieren, The Netherlands

2013

Scientific Exposure (11 ECTS)

Year

International Conferences

The First Joint International Conference of the Association of Institutions for Tropical Veterinary Medicine (AITVM) and the Society of Tropical Veterinary Medicine (STVM), Berlin, Germany

International Society for Veterinary Epidemiology and Economics

(SVEPM) Annual Conference, Inverness, Scotland

Modelling in Animal Health (ModAH) 2017, Nantes, France

\section{Seminars and Workshops}

Mini-symposium: How to Write a World-class Paper, Wageningen,

The Netherlands

Wageningen Evolution \& Ecology Seminar (WEES) on Two Frontiers

in the Study of Adaptive Significance of Animal Personalities,

Microbial Ecosystems as Medicine, and Broad Spectrum of Foraging and Migration Strategies in an Omnivorous Seabird, Wageningen,

The Netherlands

Epidemiological meetings, Lelystad, The Netherlands

The 28th Annual Meeting of the Dutch Society for Veterinary Epidemiology and Economics (VEEC), Lelystad, The Netherlands

A mini-symposium 'Mycobacterial Infections: New Methods and Insights', Wageningen, The Netherlands

\section{Presentations}

Poster presentation on modelling lumpy skin disease transmission In Ethiopia at WIAS Science Day, Wageningen, The Netherlands

Oral presentation on economic impact of lumpy skin disease and cost effectiveness of vaccination for the control of LSD outbreaks in Ethiopia at First Joint AITM-STVM Conference, Berlin, Germany Oral presentation on temporal and spatial distribution of lumpy skin Disease outbreaks in Ethiopia (2000 - 2015) at 28th VEEC Symposium, 
Lelystad, The Netherlands

Oral presentation on efficacy of Kenyan sheep pox virus vaccine for controlling lumpy skin disease outbreaks and reducing disease severity at WIAS Science Day, Wageningen, The Netherlands

Poster presentation on seroprevalence and risk factors of lumpy skin disease in Ethiopia at SVEPM Annual Conference, Inverness, Scotland

Poster presentation on transmission dynamics of lumpy skin disease in Ethiopia at Modelling in Animal Health (ModAH) 2017 Conference, Nantes, France

2017

In-Depth Studies (25 ECTS)

Year

Disciplinary and Interdisciplinary Courses

Mathematical Modelling of Infectious Diseases, Utrecht University,

The Netherlands

2014

Advanced Statistics Courses

Design of Experiment, Wageningen, The Netherlands 2013

Statistics for the Life Sciences, Wageningen, The Netherlands 2014

Generalized Linear Model, Wageningen, The Netherlands 2014

MSc Level Courses

Economics of Animal Health and Food Safety, BEC-52806 2013

Quantitative Veterinary Epidemiology, QVE-30306 2014

Management of Infections and Diseases in Animal Populations,

QVE-30806

2014

Professional Skills Support Courses (6 ECTS) Year

High-Impact Writing in Science, Wageningen, The Netherlands 2013

Techniques for Writing and Presenting a Scientific Paper,

$\begin{array}{ll}\text { Wageningen, The Netherlands } & 2014\end{array}$

Information Literacy including EndNote Introduction (ILP),

Wageningen, The Netherlands 2014

Data Management (DM), Wageningen, The Netherlands 2014

WIAS Course the Final Touch: Writing the General Introduction

And Discussion, Wageningen, The Netherlands 2016

Course Techniques for Scientific Writing, Wageningen, The Netherlands 2017 
Research Skills Training (6 ECTS) Year

Preparing own PhD Research Proposal 2013

Didactic Skills Training (1 ECTS) Year Supervising Theses

BSc Thesis 2014

Education and Training Total (52 ECTS) 
Acknowledgements 


\section{Acknowledgments}

Getting the opportunity to work my PhD at Quantitative Veterinary Epidemiology of Wageningen University and Research was a great privilege for me. This was possible with a financial support from NUFFIC fellowship. At this moment, I would like to thank NUFFIC, The Hague for the valuable financial support. I also indebted to WIAS for granting me admission to its PhD program and University of Gondar for allowing me this study leave. Completing my thesis would have not been possible without the support of many people. I would like to use this opportunity to thank all the people that have helped me along the way.

First, and foremost, I would like to acknowledge my promotor prof. Dr ir. Mart C.M. de Jong and my daily supervisor Dr ir. Klaas Frankena, who provided the inspiration and guidance that was necessary to proceed through my PhD program. Mart, thank you very much for your confidence in me and giving me the opportunity to do my PhD under your supervision. Your contribution on my PhD journey was great; particularly your constructive criticism and enlightening discussions worth to mentioned. Klaas, this PhD would not have been a success without your untiring support. I very much appreciate your devotion to review my papers repeatedly and meticulously. I learned a lot from your critical review, data analysis and scientific writings, thank you for being on the side of me all the time. I really thank both of you for your all round support during my stay at Wageningen. Thank you Dr Getachew Gari for agreeing to be my local supervisor, and your encouragements and all-round support during the field and laboratory works.

I would like to extend my gratitude to Dr Mesfin Sehale, director of National Animal Health Diagnostic and Investigation Centre, Dr Martha Yami, General Manager of NVI, Dr Bewket Siraw, director of Veterinary Services Directorate and Dr Gelagay Ayelet, Vaccine Production and Sales Core Process Owner at NVI whose encouragement and logistic support during field data collection and laboratory testing has been a crucial input for the successful accomplishment of my research. I am also grateful to Dr Yesmashewa Wogayehu for facilitating outbreak data base access at Veterinary Services Directorate and Dr Samuel for arranging transport for the field work.

Lisette and Ada, thank you very much for your help at various times. Really, I enjoyed your kindness and support for any administrative issues I had in your office. Indeed you have been on my sides both in times of stress and happiness. I am grateful to all PhD colleagues at QVE and ABG groups who made my stay pleasant. Floor, thanks a lot for your support during my stay in Wageningen, particularly in helping me with administrative issues. 
I am grateful to Aleghegn Yeshiwas, Alehegn Anmaw, Adamtachew Mekonnen, Endegena Abebe, Dr Adugna, Asheber Abebe, Tafes Koran, Menbere Kidane, Dr Dereje Shegu, and all staffs of NAHDIC for their assistance during data and sample collection in the field and sample processing in the laboratory. Dr Gizat Almaw, thank you very much for your support and friendship while I was at NAHDIC.

I would also like to thank colleagues at University of Gondar for their encouragement and support during my PhD study. Dr Wudu, thank you very much for your encouragement and assistance from the inception to the end of my PhD project. I have really enjoyed your friendship in our home town and Wageningen.

Never would this have been possible without the support and understanding of my family. Bethelihem Mesfin, my love, you have done indeed an excellent job by taking care of our kids while I was away. I thank you very much for your encouragement, love and allowing me to undertake this study. My kids Yosef, Tsion and Amanuel, you are my blessing, thank you so much being the source of my excitement and extremely patient with me.

I indebted to everyone who contributed in one way or the other in the accomplishment of this thesis. It is difficult to mention by name, but I am sincerely acknowledge your contribution. 


\section{Colophon}

The research described in this thesis was financially supported by The Netherlands Organization for International Cooperation in Higher Education (NUFFIC).

Cover picture taken by Wassie Molla Abebe 